\title{
Micropollutant degradation in water by photochemical processes
}

Yin Ye

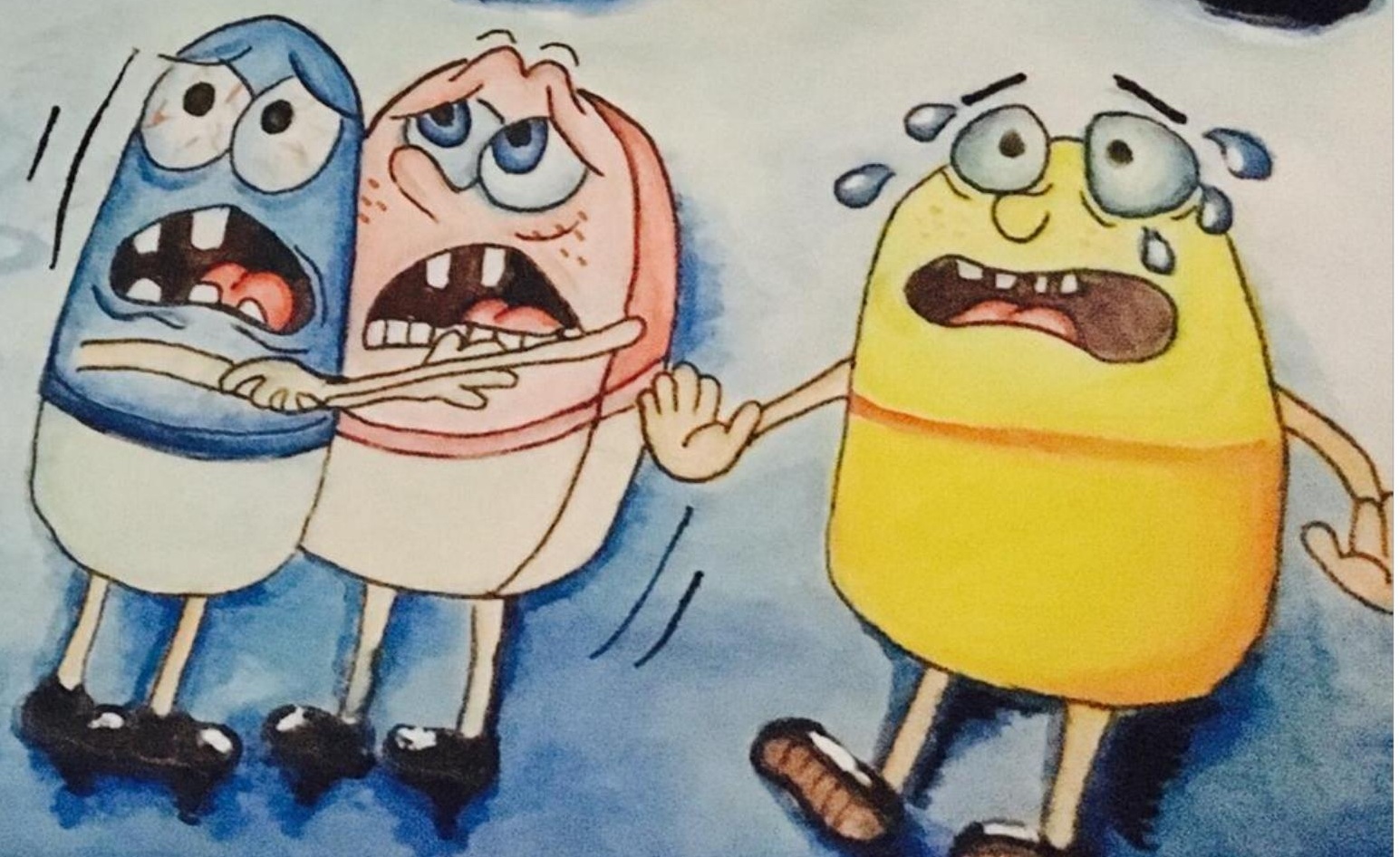





\section{Propositions}

1. $\mathrm{TiO}_{2}$ nanotube array is a promising immobilized photocatalyst for the removal of micropollutants.

(this thesis)

2. Red light induced methylene blue photosensitization provides an efficient process for micropollutants removal.

(this thesis)

3. Innovation and open-mindedness are founding elements of scientific research.

4. Internet is a catalyst of scientific research.

5. The Dutch style doctoral education is the best in the world.

6. The business model of the current academic publishing industry is the smartest in the world.

7. For peace, communication and empathy are needed.

Propositions belonging to the thesis, entitled

"Micropollutant degradation in water by photochemical processes"

Yin Ye

Wageningen, 7 December 2018 



\section{Micropollutant degradation in water by photochemical processes}

Yin Ye 


\section{Thesis committee}

\section{Promotor}

Prof. Dr H.H.M. Rijnaarts

Professor of Environmental Technology

Wageningen University \& Research

\section{Co-promotors}

Dr H. Bruning

Assistant Professor, Sub-department of Environmental Technology

Wageningen University \& Research

Dr D. Yntema

Scientific Project Manager

Wetsus, European Centre of Excellence for Sustainable Water Technology, Leeuwarden

\section{Other members}

Prof. Dr D. Dionysiou, University of Cincinnati, USA

Prof. Dr J.T. Zuilhof, Wageningen University \& Research

Prof. Dr P.M. Heynderickx, Ghent University, Belgium

Dr R. Hofman, KWR, Nieuwegein

This research was conducted under auspices of the Graduate School for SocioEconomic and Natural Sciences of the Environment (SENSE) 


\title{
Micropollutant degradation in water by photochemical
}

processes

\author{
Yin Ye
}

Thesis

submitted in fulfilment of the requirements for the degree of doctor at Wageningen University

by the authority of the Rector Magnificius,

Prof. Dr A.P.J. Mol,

in the presence of the

Thesis Committee appointed by the Academic Board

to be defended in public

on Friday 7 December 2018

at 4 p.m. in the Aula. 
Yin Ye

Micropollutant degradation in water by photochemical processes, 238 pages.

PhD thesis, Wageningen University, Wageningen, the Netherlands (2018) With references, with summary in English

ISBN 978-94-6343-317-4

DOI https://doi.org/10.18174/456013 


\section{To my beloved family}

谨以此书献给我的父母、妻子和孩子 



\section{Contents}

Chapter 1. General introduction

Chapter 2. Photocatalytic degradation of metoprolol by $\mathrm{TiO}_{2}$ nanotube arrays and UV-LED: effects of catalyst properties, operational parameters, commonly present water constituents, and photo-induced reactive species 23

Chapter 3. Effect of dissolved natural organic matter on the photocatalytic micropollutant removal performance of $\mathrm{TiO}_{2}$ nanotube array . 63

Chapter 4. Significant enhancement of micropollutant photocatalytic degradation using a $\mathrm{TiO}_{2}$ nanotube array photoanode based photocatalytic fuel cell 85

Chapter 5 . Homogeneous photosensitized degradation of pharmaceuticals by using red light LED as light source and methylene blue as photosensitizer 115

Chapter 6. Operational parameters affecting MB/Red-light photosensitized degradation of pharmaceuticals 147

Chapter 7. General discussion 169

Bibliography 185

List of abbreviations 219

Summary 221

Acknowledgements 227

About the author 231

List of publications 233 

Chapter 1

General introduction

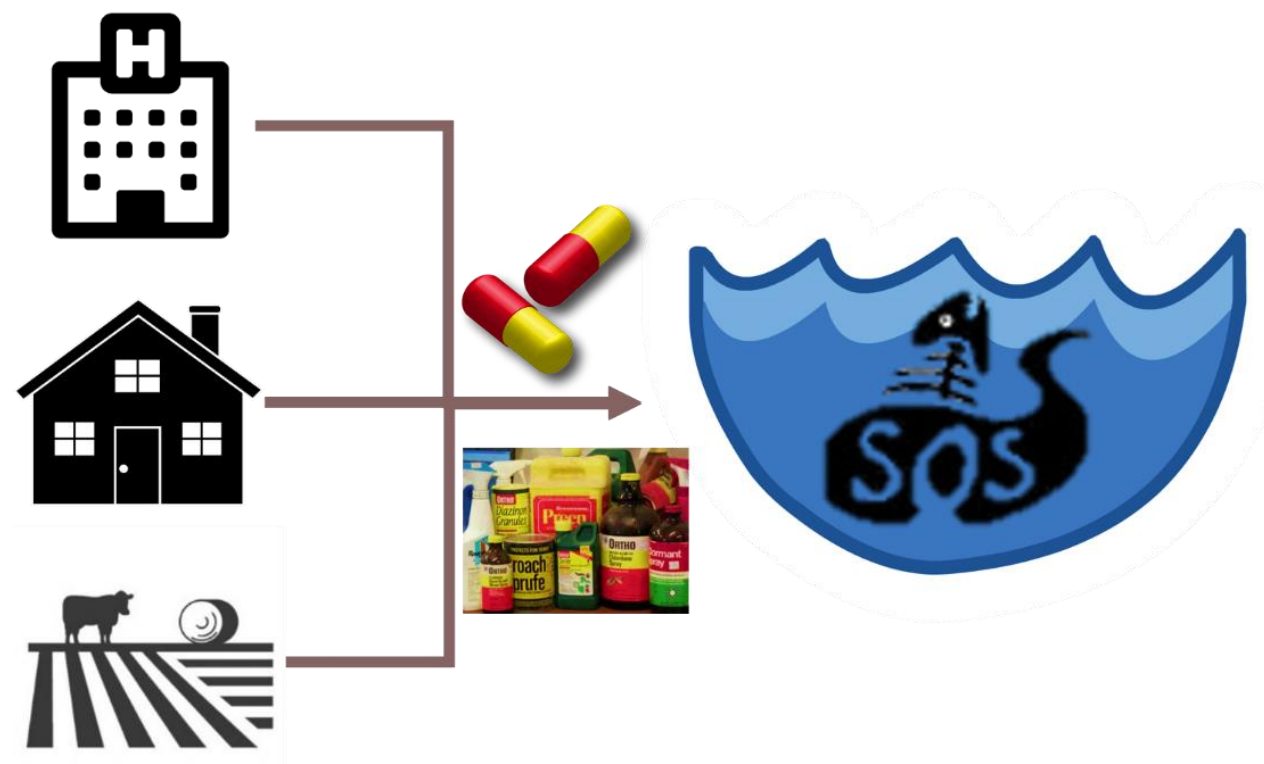




\section{Chapter 1}

\subsection{The challenge of micropollutants: occurrence in aquatic environment, and}

risks

Fast growing global population and economy result in an increasing water consumption worldwide. However, the amounts of usable water resources are limited: more than one-third of the accessible renewable fresh water on Earth is being exploited for industrial, agricultural, as well as domestic usage [1]. Locally, especially in water scarce areas, this is often reaching much higher levels, leading to overdraft of water resources [2]. Such facts call for the need to not only exploit new and alternative water resources but also to recycle water streams, to cope with the unfortunate increasing limitations in availability of fresh water sources. Meanwhile, the fast growing population and economy are leading to serious water pollution due to discharge of numerous contaminants into the environment, which in turn possess sever threats to the quality of water resources. Thus, the increasing worldwide demand for water consumption, the limited amount of water resources, and the water pollution make water treatment a vital measure to safeguard the water supply with sufficient quantity and quality that meets the demand of the society. Conventionally, concerns were given to pathogens, nutrients, heavy metals, suspended solids, and bulk organic pollutants (the amount of bulk organic pollutants generally present in concentrations at $0.1-10 \mathrm{~g} / \mathrm{l}$ in waste water and often denoted with COD or BOD, meaning chemical or biological oxygen demand). Thus in most parts of the world water purification measures have been taken to tackle these problems $[3,4]$. In recent decades, many emerging organic pollutants (generally present at rather low concentrations, i.e. $\mathrm{ng} / \mathrm{L}$ to $\mu \mathrm{g} / \mathrm{L}$ level, and therefore also termed as micropollutants). These include pharmaceuticals, antibiotics, herbicides, pesticides, chemicals from personal care products, etc., and have been detected the last two decades extensively in different water bodies worldwide. For instance, many pharmaceuticals were found in various portions of the aquatic system, i.e. in ground water, surface water, as well as in many drinking water sources according to a broad range of studies [5-11]. In a study conducted by Félix-Cañedo et al. in Mexico City, the presence of a group of organic micropollutants (including pharmaceuticals, hormones, herbicides) in several drinking water sources was reported, at concentrations ranging from $\mathrm{ng} / \mathrm{L}$ to $\mu \mathrm{g} / \mathrm{L}$ level [12]. In recent years, a broad range of micropollutants, including 
pesticides, pharmaceuticals, industrial compounds, estrogens, chemicals originating from life-style compounds and personal care products, have been measured in groundwater in Spain, according to a review paper by Jurado et al. [13]. The occurrence of micropollutants in aquatic environment has become a worldwide issue that is gaining increasing concerns $[7,14-17]$.

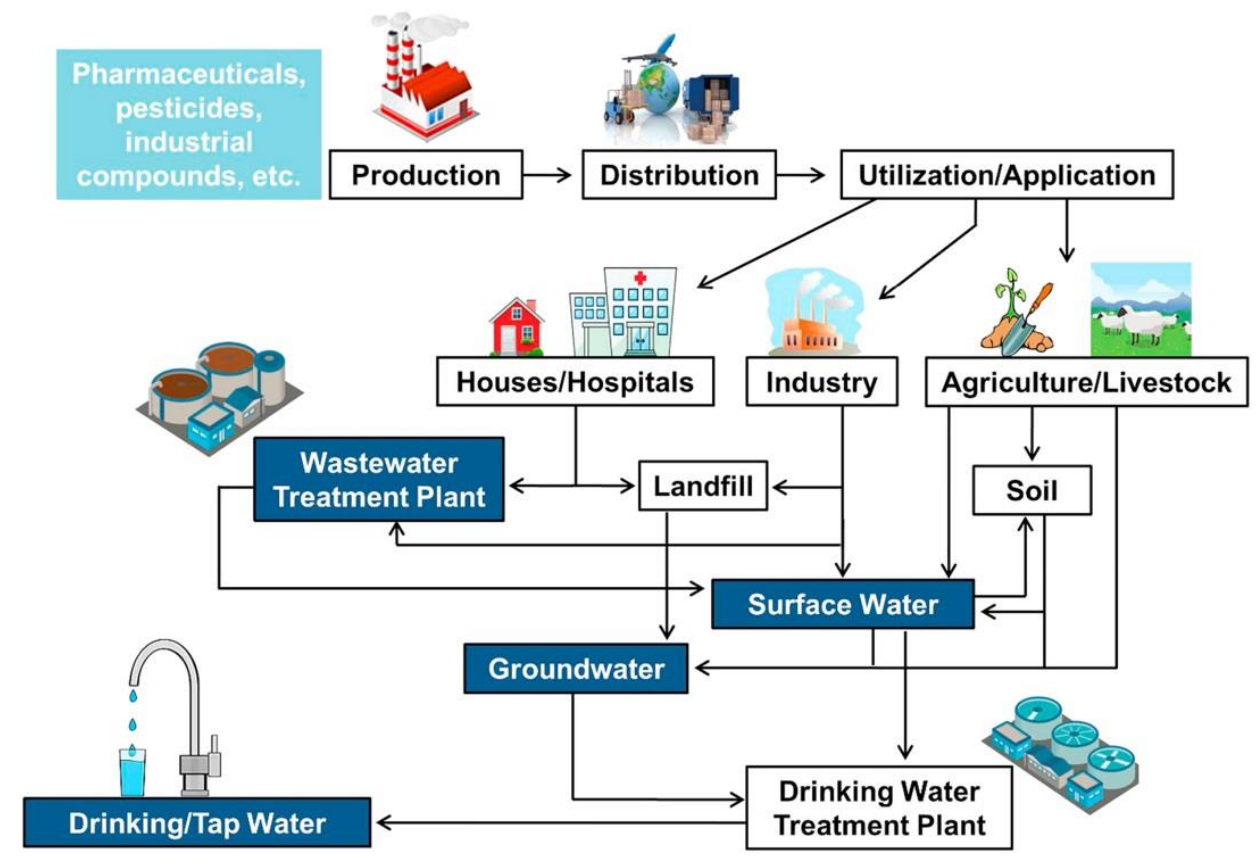

Figure 1. 1 Typical sources of micropollutants entering the environment and their migration routes into drinking water (Figure from [18] with permission).

Micropollutants enter the environment from various sources and sometimes end up in drinking water via various migration pathways, as illustrated in Figure 1. 1 [18]. Micropollutants can be introduced into the environment via discharge of industrial effluents of pharmaceuticals, personal care products, pesticides and other synthetic compounds manufacturing plants. Usage and disposal of pharmaceuticals, antibiotics, personal care products, etc., in households and hospitals are another major source of those compounds [19]. The conventional municipal wastewater treatment plants (WWTP) were originally designed for bulk organics and nutrient removal, and not for the removal of micropollutants. The fate of micropollutants in WWTPs can therefore vary greatly. For individual 


\section{Chapter 1}

micropollutant, its removal efficiency greatly relies on the operation conditions and treatment technology applied in specific WWTP, and can differ drastically among different WWTPs [18]. For individual WWTP, its removal efficiency on micropollutants greatly relies on the properties of specific compound, e.g. adsorption ability to solids, biodegradability, etc., [17]. Moreover, in a review study by Luo et al., it was found that the WWTP removal efficiency of 39 most studied micropollutants in 14 countries/regions showed a clear variation, which can be ascribed to both micropollutant properties and WWTP operational conditions [17]. Although it is hard to draw a firm conclusion of persistency of each micropollutant in WWTPs, many compounds are suggested to be poorly removed in WWTPs, e.g. carbamazepine, diclofenac, metoprolol, etc., [17]. Nevertheless, the insufficient removal of micropollutants in WWTPs makes WWTPs effluent the main cause of micropollutants presence in aquatic environment [20]. Additionally, runoff from agriculture, livestock, and aquaculture is another major source that brings micropollutants (mainly pharmaceuticals, herbicides, and pesticides) into the environment.

Once entering the environment, micropollutants may undergo different transformation/migration pathways and have thereby varied fates, depending on their physical/chemical properties. Sorption onto suspended solids and sediments, direct and indirect photolysis and aerobic biodegradation are the typical attenuation processes that many micropollutants may undergo in the environment [16]. Photodegradation, including direct and indirect photolysis, is a major set of transformation pathways of micropollutants in water bodies. For instance, carbamazepine can undergo direct photolysis, and its photochemical half-life in surface waters ranges from weeks to several months, depending on the environmental conditions [21]. Sorption onto suspended solids and sediments is a major migration pathway of hydrophobic micropollutants. Diphenhydramine was found to sorb onto aquatic sediments and can thus be concentrated to as much as one thousand times over its aqueous concentration [22]. More comprehensive studies on the fate and transformation of micropollutants in aquatic system are available in literature $[13,16-18$, 23-25].

Although the presence of micropollutants in aquatic systems is at trace concentrations ranging from $\mathrm{ng} / \mathrm{L}$ to $\mu \mathrm{g} / \mathrm{L}[1,17,18,26]$, undesirable effects on the 
ecosystems have been reported by many studies. These point out that those contaminants present at low concentrations impose potential hazards to aquatic life at different levels and species varying from algae to fish [27]. Detrimental effects including toxicity on living species, endocrine disrupting effects on organisms, and building up of antibiotic resistance in pathogens, are abundantly and frequently reported [28-31]. For instance, it was shown that diclofenac is toxic to three Gyps vulture species [32], and the exposure to diclofenac residues in the environment was found to be responsible for vulture population decline in Pakistan in another study [33]. Significant correlations between antibiotic resistant bacteria and corresponding antibiotic concentrations in a municipal wastewater treatment plant were reported by Gao et al. [34], which suggested that presence of antibiotics in the environment would contribute to the development of antibiotic resistance genes in bacteria. Human exposure to micropollutants is also gaining increasing concerns, because many micropollutants can accumulate in the food chain. It was reported that micropollutants in the agricultural irrigation network could be taken up by crops and later on by humans via consumption of fruits and vegetables [35]. That study found that the hydrophobic micropollutants could easily accumulate in crops, and the estimated human exposure to micropollutants through fruit and vegetable consumption was about $9.8 \mu \mathrm{g}$ per person per week. In addition, the presence of micropollutants in drinking water provides a direct exposure route to humans [36]. Although acute human toxicity of many micropollutants is not expected $[37,38]$, the human health effects upon long-term exposure to such pollutants is not fully understood and the development of an appropriate standard assessment methodology is urgently wanted [39, 40], some studies did already indicate that human health risks can be caused by exposure to certain micropollutants. For well-known industrial and pesticide chemicals, (Like HCB, dieldrin, chlordane, DDTs and PCBs) the cancer risks caused by daily exposure due to fish consumption has been reported extensively [41]. Such effects are not yet adequately studied for the large groups of pharmaceuticals and personal care product related chemicals. Moreover, although some individual compounds might not impose acute toxicity at the low concentrations appearing in the environment, micropollutants are often present in water bodies as a complex mixture which may cause additive and synergetic toxicity effects on living organisms [42]. For instance, studies have demonstrated such synergetic detrimental effect of mixtures of estrogenic chemicals on 


\section{Chapter 1}

freshwater and seawater fishes [43, 44]. Growing research efforts have been directed into this field [45, 46]. Given this context, authorities are taking measures to establish regulations on this issue. For instance, a watch list of priority substances including several micropollutants has been published by the European Union [47].

The increasing legislation pressure, risks associated with micropollutants, and the insufficient efficacy of conventional WWTPs on micropollutant removal have formed a joint driving force that is boosting studies on more efficient treatment technology, removing micropollutants from wastewater and water sources used for drinking water. Photochemical treatment, the focus of this thesis, forms a class of techniques using light to degrade micropollutants.

\subsection{Photochemical treatment techniques for micropollutant removal}

Conventional water treatment processes, e.g. biological processes, sedimentation, filtration, coagulation/flocculation, are designed for elimination of solid suspends, carbonaceous substances, nutrients, pathogens. Although those contaminants can usually be effectively removed within conventional water treatment processes, the removal of micropollutants is usually unfortunately insufficient [23]. Therefore, vast efforts have been made to develop more advanced treatment processes for this purpose, e.g. activated carbon adsorption, ozonation, constructed wetlands, etc., [48-54]. The photochemical techniques are, among other (biological, sorptive, and oxydative-chemical) technologies, currently strongly under development to remove organic micropollutants from water. The advantage of photochemical techniques is that these generally do not need the addition of microorganisms to the water, and therefore are very much appreciated by drinking water companies and water boards, the organisations that implement most water treatment technologies. Among all the treatment technologies for micropollutant removal, photochemical processes have therefore drawn enormous attention in recent decades. So far, various photochemical processes have been studied as removal techniques for different micropollutants, such as $\mathrm{UV} / \mathrm{H}_{2} \mathrm{O}_{2}$ [55-59], UV/TiO 2 [60-62], Photo-Fenton [63], photosensitization $[64,65]$, etc., which rely greatly on generation of various reactive oxygen species (ROS). Most commonly studied processes are: (1) UV/ $\mathrm{H}_{2} \mathrm{O}_{2}$; (2) Photo-Fenton; (3) UV photolysis; (4) Photocatalysis; (5) Photosensitization. 


\subsubsection{UV/ $\mathrm{H}_{2} \mathrm{O}_{2}$ process}

The $\mathrm{UV} / \mathrm{H}_{2} \mathrm{O}_{2}$ process relies on photolysis of $\mathrm{H}_{2} \mathrm{O}_{2}$ molecules under UV irradiation, which leads to hydroxyl radical $(\cdot \mathrm{OH})$ generation, as illustrated in the reaction below:

$$
\mathrm{H}_{2} \mathrm{O}_{2} \stackrel{\mathrm{hv}}{\rightarrow} 2 \cdot \mathrm{OH}
$$

The produced hydroxyl radicals $(\cdot \mathrm{OH})$ possess high oxidizing power $(2.8 \mathrm{~V})$, which gives them strong ability to capture electrons from other compounds, and therefore are capable to subsequently degrade organic pollutants, including micropollutants. In recent decades, $\mathrm{UV} / \mathrm{H}_{2} \mathrm{O}_{2}$ processes have drawn large amount of research interests in the field of micropollutant removal, and examples are ubiquitous [66]. For instance, Wols et al. studied degradation of 40 selected pharmaceuticals and reported good removal efficiency for most of the pharmaceuticals studied [67]. It is reported that a combination of $122.5 \mathrm{~kJ} / \mathrm{m}^{2} \mathrm{UV}$ radiation and $4 \mathrm{mg} / \mathrm{L} \mathrm{H}_{2} \mathrm{O}_{2}$ could lead to more than $90 \%$ removal of estriol, $17 \beta$-estradiol and $17 \alpha$-ethinylestradiol, in secondary WWTP effluent [68]. In another study conducted by Shu et al. [69], good removal efficiency of 2,4-D and carbamazepine was presented in a pilot-scale $\mathrm{UV} / \mathrm{H}_{2} \mathrm{O}_{2}$ reactor. Removal of other micropollutants including estrone, ibuprofen, diphenhydramine, phenazone, phenytoin, clarithromycin, diclofenac, metoprolol, benzotriazole, mecoprop, metaldehyde, etc., by $U V / \mathrm{H}_{2} \mathrm{O}_{2}$ processes have also been reported, and the removal efficiency varied among studies due to operation under different conditions [59, 70-72]. At present, the $\mathrm{UV} / \mathrm{H}_{2} \mathrm{O}_{2}$ technique is already commercially available as a disinfection tool and micropollutants barrier, pilot to full scale application examples can be found elsewhere $[73,74]$.

\subsubsection{Photo-Fenton processes}

The principle of Fenton based processes relies on iron $\left(\mathrm{Fe}^{2+}\right)$ catalysed hydrolysis of $\mathrm{H}_{2} \mathrm{O}_{2}$, which results in production of $\cdot \mathrm{OH}$, as illustrated in the equations below [75]:

$$
\begin{aligned}
& \mathrm{H}_{2} \mathrm{O}_{2}+\mathrm{Fe}^{2+} \rightarrow \mathrm{Fe}^{3+}+\mathrm{OH}^{-}+\cdot \mathrm{OH} \\
& \mathrm{H}_{2} \mathbf{O}_{2}+\mathrm{Fe}^{3+} \rightarrow \mathrm{Fe}^{2+}+\mathrm{H}^{+}+\cdot \mathrm{HO}_{2}
\end{aligned}
$$

By applying UV radiation, thus in photo-Fenton processes, the $\cdot \mathrm{OH}$ generation is enhanced: (1) regeneration of $\mathrm{Fe}^{2+}$ is accelerated; (2) photolysis of $\mathrm{H}_{2} \mathrm{O}_{2}$ would also 


\section{Chapter 1}

contribute to $\mathrm{OH}$ production. Therefore photo-Fenton process is much more efficient in the $\cdot \mathrm{OH}$ generation compared with the conventional Fenton process $[76,77]$. Viewing this fact, many studies have been performed to employ photo-Fenton technique for micropollutant removal, and removal efficiency depends greatly on the $\mathrm{pH}$ of reaction solution, $\mathrm{Fe}^{2+}$ and $\mathrm{H}_{2} \mathrm{O}_{2}$ dosages [63, 76, 78-82]. Usually, acidic $\mathrm{pH}$ (lower than 3 ) is required in Fenton processes to obtain good solubility of the iron ions [83], which is one of the main drawbacks of Fenton based techniques. Although efforts have been taken to extend the bearable $\mathrm{pH}$ to neutral, additional Fe stabilization reagents, e.g. ethylenediamine- $N, N^{\prime}$ disuccinic acid, are still needed [84]. Additionally, the production of iron sludge is a major concern of the Fenton based processes, due to precipitation of $\mathrm{Fe}(\mathrm{OH})_{3}$, which calls for extra measures for proper disposal/treatment of the sludge [85].

\subsubsection{UV photolysis}

Due to the high energy of UV photons, they are able to trigger cleavage of various chemical bonds and elevation of molecular energy once absorbed by chemicals, making UV radiation capable to decompose various chemical structures. There are two main mechanisms, i.e. direct and indirect photolysis, responsible for chemical decomposition under UV radiation. The direct photolysis of an organic compound is the consequence of that compound's own light absorption [86], while indirect photolysis of an organic compound involves participation of ROS generated by the interaction between photons and other co-existing chemicals [87]. Since UV treatment is one of the main disinfection techniques used in WWTPs [88] and many micropollutants can undergo direct or indirect photolysis upon UV (mainly in or shorter than UV-C range) radiation, it is possible to achieve micropollutant removal during UV disinfection processes. Viewing this, direct UV radiation has been one of the most widely studied micropollutant removal techniques, examples are well documented in literature [89-92]. For instance, in a recent study conducted by a group of Spanish researchers, it has been reported that UV treatment would be capable for the removal of several micropollutants [93]. It was reported that, among 18 tested micropollutants, a lab-scale UV reactor (lamp emitting at $254 \mathrm{~nm}$ ) enabled effective removal of diclofenac, fluoxetine, sulfamethoxazole, bisphenol A, estrone, $17 \beta$-estradiol, and $17 \alpha$ ethynylestradiol. The removal of erythromycin, roxithromycin, carbamazepine, 
trimethoprim, and diazepam was more limited and more affected by the operational conditions. These authors also found that the low UV dose applied for disinfection in fullscale WWTPs was insufficient for effective and complete removal of the micropollutants, although some compounds were degraded. A good overview of the studies on the removal of micropollutants by direct UV radiation is available in literature [94].

\subsubsection{Photocatalytic processes}

The report of Fujishima and Honda in 1972 on photoelectrochemically conversion of photo energy into chemical energy inspired vast research interests in photocatalysis [95]. Although many semiconductors (e.g. $\mathrm{ZnO}$ [96-99], $\mathrm{WO}_{3}$ [100], etc. ) have photocatalytic activity, $\mathrm{TiO}_{2}$ is the most used photocatalyst in environmental applications including air pollution control [101] and water treatment [102], because of its low toxicity, low cost, high efficiency, and high stability. Principles of $\mathrm{TiO}_{2}$ photocatalytic processes have been described elsewhere $[62,103]$. $\mathrm{TiO}_{2}$ has three crystalline phases, thus rutile, anatase, and brookite. For photocatalytic water treatment purposes, rutile and anatase are the two favourable crystalline phases because of their appropriate band gaps (3.2 eV for anatase and $3.0 \mathrm{eV}$ for rutile) [104]. As illustrated in Figure 1. 2, the photocatalytic degradation of organic pollutants is usually initiated by irradiation of photons containing energy higher than the band gap of the catalyst (UV photons in this case). Sequentially, the electrons $\left(\mathrm{e}^{-}\right)$ in the valence band of $\mathrm{TiO}_{2}$ would be excited to the conduction band and leave positively charged holes $(\mathrm{h}+)$ in the valence band, and in this way $\mathrm{e}^{-} / \mathrm{h}^{+}$pairs are generated. The photogenerated electrons and holes would then migrate to the $\mathrm{TiO}_{2}$ surface and participate in series of redox reactions. Both the electrons and holes are able to directly react with target organic contaminants. Besides, these photo-generated electrons and holes can also react with oxygen, water, or $\mathrm{HO}^{-}$to generate highly reactive species, e.g hydroxyl radicals $(\cdot \mathrm{OH})$, superoxide radical anions $\left(\mathrm{O}_{2} \cdot^{-}\right)$, etc., and those in-situ generated reactive oxygen species (ROS) are able to oxidize a broad range of organic contaminants and inactivate pathogens. Meanwhile, part of the $\mathrm{e}^{-/ \mathrm{h}^{+}}$pairs would also undergo recombination without participation in redox reactions, which causes waste of irradiation energy and is a major problem to photocatalysis processes. 


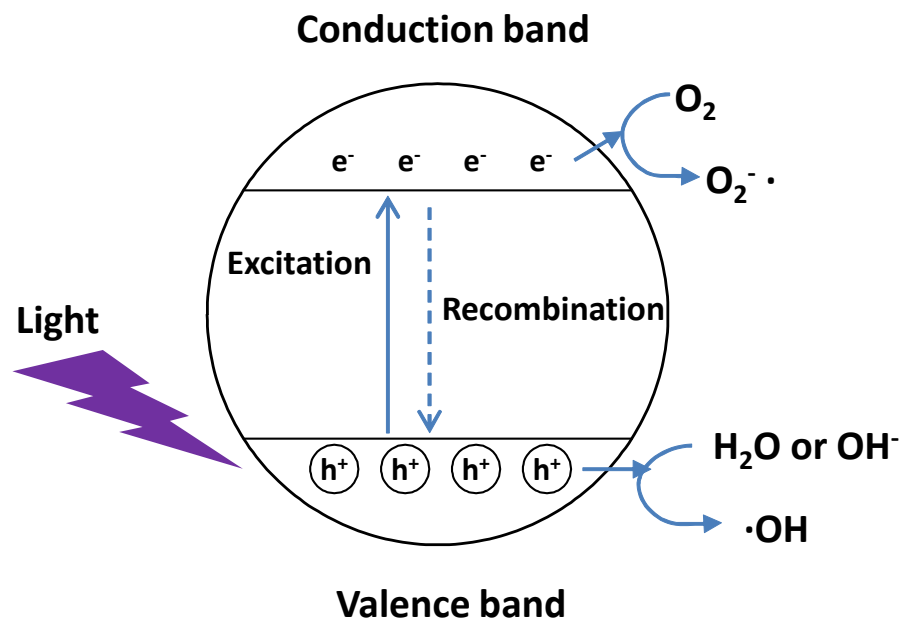

Figure 1. 2 Schematic representation of principles of photocatalytic processes

Applicability of $\mathrm{TiO}_{2}$ for elimination of a wide range of organic contaminant has been well documented [105-109]. In recent decade, many studies have been done to apply $\mathrm{TiO}_{2}$ for photocatalytic removal of various micropollutants [110-114], e.g. 2-methyl-4chlorophenoxyacetic acid (MCPA) [115-117], mecoprop [118], clopyralid [118], metoprolol [104, 119], 2,4-Dichlorophenoxyacetic Acid (2,4-D) [120], etc. For instance, Calza et al. [61] reported efficient diclofenac removal over aqueous $\mathrm{TiO}_{2}$ suspensions. Although $\mathrm{TiO}_{2}$ photocatalysis has been shown to be an effective way to remove a variety of micropollutants from water by lab-scale studies, there are still two major issues need to be addressed. The first one is separating and recycling of the $\mathrm{TiO}_{2}$ prior to the downstream treatment processes. One option is the membrane filtration. By combining the $\mathrm{TiO}_{2}$ photocatalysis processes with membrane filtration technique, good retention of $\mathrm{TiO}_{2}$ can be expected. For example, Benotti et al. [121] evaluated the performance of a pilot-scale photocatalytic membrane reactor (consisting of a photocatalytic reaction chamber and a cross flow ceramic membrane unit where $\mathrm{TiO}_{2}$ nanoparticles were separated and recycled) on the removal of 32 pharmaceuticals and endocrine disrupting compounds in river water. Their results showed good micropollutant removal performance and $\mathrm{TiO}_{2}$ retention. However, the application of membrane separation would raise new concerns like additional operation cost and fouling. An alternative is applying immobilized $\mathrm{TiO}_{2}$ instead of $\mathrm{TiO}_{2}$ 
suspensions. For instance, in a study conducted by He et al. [122], good degradation performance of propranolol, diclofenac, carbamazepine and ibuprofen over $\mathrm{TiO}_{2}$ immobilized sand was reported, and additional post treatment for catalyst separation was avoided. Another major shortcoming of $\mathrm{TiO}_{2}$ photocatalysis technique is the recombination of $\mathrm{e}^{-} / \mathrm{h}^{+}$pairs. As aforementioned, upon generation of $\mathrm{e}^{-/ h^{+}}$pairs, part of them would not participate in any redox reactions and would recombine to generate heat. Such process do not contribute to degradation of target contaminants, instead, it only leads to loss of absorbed photon energy, and therefore lowering the energetic efficiency of the $\mathrm{TiO}_{2}$ photocatalysis. Thus, suppressing the $\mathrm{e}^{-} / \mathrm{h}^{+}$recombination is a key to improve the performance of $\mathrm{a} \mathrm{TiO}_{2}$ photocatalysis technique. Up till know, several approaches have been investigated for this purpose. For instance, modification of $\mathrm{TiO}_{2}$ by adding electron acceptor species $\left(\mathrm{S}_{2} \mathrm{O}_{8}{ }^{2-}\right)$ to trap photogenerated electrons is reported to be effective in reducing the recombination of $\mathrm{e}^{-} / \mathrm{h}^{+}$pairs and enhancing the degradation of sixteen substituted phenylurea herbicides [123]. Another approach is extraction of photogenerated electrons by applying external voltage. By applying an external voltage, the photogenerated electrons can be effectively extracted from the catalysts, and thus recombination of $\mathrm{e}^{-} / \mathrm{h}^{+}$can be greatly suppressed, so better target pollutants degradation can be expected [124, 125].

\subsubsection{Photosensitization processes}

The term photosensitization refers to "the process of initiating a reaction through the use of a substance capable of absorbing light and transferring the energy to the desired reactants." [126]. Figure 1. 4 shows the principles of photosensitized pollutants removal processes. In a typical photosensitization process, the photosensitizer absorbs energy from photons to become in excited state. Then the excited state photosensitizer can react with oxygen to form singlet oxygen $\left({ }^{1} \mathrm{O}_{2}\right)$ via energy transfer or superoxide anion radical $\left(\cdot \mathrm{O}_{2}{ }^{-}\right)$via electron transfer, which is defined as Type II photosensitization; alternatively, the excited state photosensitizer can also reacts directly with the pollutants via electron transfer, which is defined as Type I photosensitization [127, 128]. Many compounds have been recognised to be able to act as photosensitizers, including organic dyes, transition metal complexes, semiconductors, and porphyrins and their analogues [127]. Singlet oxygen, an energetically rich form of molecular oxygen, is considered to be the primary oxidant in various 


\section{Chapter 1}

photosensitization processes [64, 129, 130]. Figure 1. 3 illustrates the difference between ground state oxygen and singlet oxygen in the $\pi$-antibonding orbitals structure. Singlet oxygen has different electronic configuration than the ground state oxygen (the $\pi$ antibonding electrons of the singlet oxygen lie in one orbital and have opposite spins) and higher energy level $(95 \mathrm{~kJ} / \mathrm{mol})$ than ground state oxygen. Such properties make singlet oxygen significantly more electrophilic and more reactive towards unsaturated bonds than the ground state oxygen: it reacts with singlet state electron-rich compounds unsaturated bonds, and typical reactions of singlet oxygen are Schenck reactions and Diels-Alder reactions.

\section{$\pi$-antibonding orbitals assignment}

\section{Singlet oxygen $\left({ }^{1} \mathrm{O}_{2}\right)$}

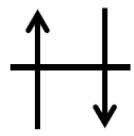

Ground state oxygen $\left({ }^{3} \mathrm{O}_{2}\right)$
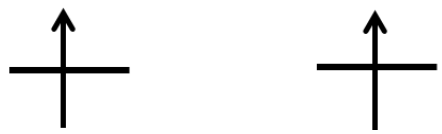

Figure 1. 3 Representations of the $\pi$-antibonding orbitals assignment of ground state oxygen and singlet oxygen

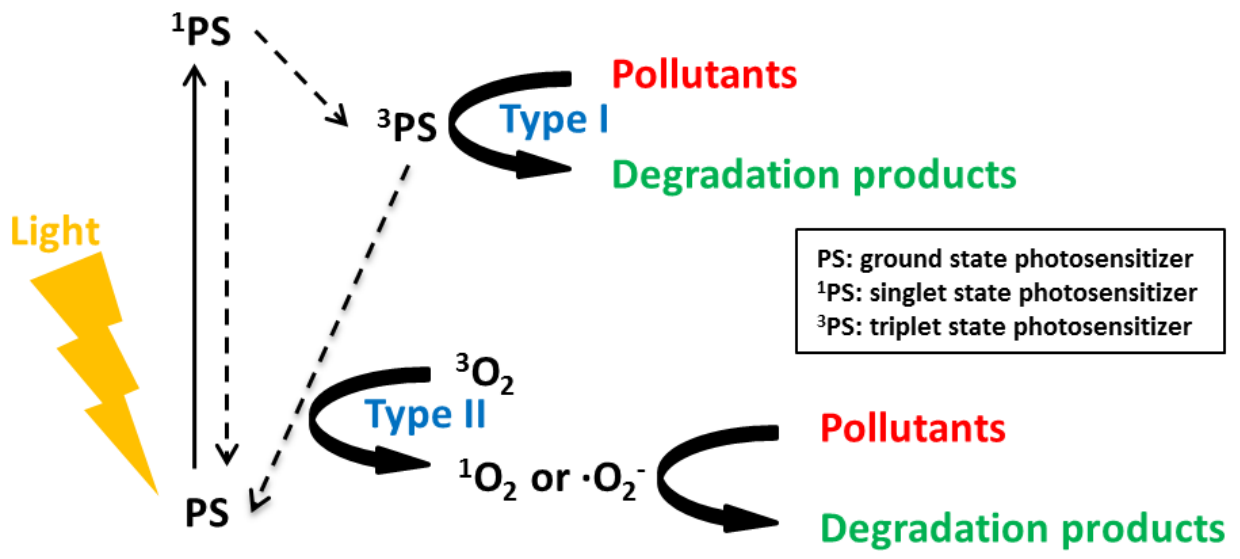

Figure 1. 4 Schematic representation of principles of photosensitized pollutants removal processes 
Photosensitization processes are one of the main transformation pathways of micropollutants attenuation in natural water bodies, because many micropollutants themselves can act as photosensitizers and the commonly present dissolved natural organic matter are known to have photosensitizing effects, but such transformation in nature is usually slow $[8,10,21]$. In recent years, studies have been carried out to employ photosensitization processes for organic chemical removal in various water matrices. For instance, Gmurek et al. examined the application of photosensitive chitosan (chitosan with immobilized phthalocyanine) for visible light (natural sunlight) photosensitized degradation (by ${ }^{1} \mathrm{O}_{2}$ ) of benzylparaben and 2, 4-dichlorophenol, the results show that the studied system had good efficacy and could work in wide $\mathrm{pH}$ range [131]. Kim et al. reported degradation of several pharmaceuticals using tin porphyrin- and C60 aminofullerene-derivatized silica as photosensitizers, where ${ }^{1} \mathrm{O}_{2}$ was identified as the primary oxidant, and the performance in real wastewater effluents was still effective [130]. The advantage of ${ }^{1} \mathrm{O}_{2}$ over other ROS such as. $\mathrm{OH}$ is its high electrophilicity and selectivity, thus ${ }^{1} \mathrm{O}_{2}$ can effectively oxidize micropollutants containing electron-rich moieties, such as alkyl sulphides and thiones, despite the presence of background organic matter [127, 129].

\subsubsection{Other processes}

Other investigated homogeneous photochemical processes include $\mathrm{UV} / \mathrm{Cl}_{2}, \mathrm{UV} / \mathrm{O}_{3}$, $\mathrm{UV} / \mathrm{HOCl}, \mathrm{UV} / \mathrm{ClO}_{2}$. Application of these techniques for removal of various micropollutants has been studied over last decades. It is reported that $\mathrm{UV} / \mathrm{Cl}_{2}$ could achieve relatively good removal of carbamazepine from wastewater, but formation of toxic chlorinated byproducts was an issue $[132,133]$. Kong et al. reported efficient degradation of atrazine by $\mathrm{UV} / \mathrm{Cl}_{2}$ [134]. Examples of micropollutant removal by $\mathrm{UV} / \mathrm{O}_{3}, \mathrm{UV} / \mathrm{HOCl}, \mathrm{UV} / \mathrm{ClO}_{2}$, etc., are also abundant in literature [135-138].

\subsection{Research opportunities}

\subsubsection{Research opportunities within existing photochemical techniques}

Although as aforementioned, vast efforts have been directed into photochemical processes for micropollutant removal. Various techniques, e.g. $\mathrm{UV} / \mathrm{Cl}_{2}, \mathrm{UV} / \mathrm{O}_{3}, \mathrm{UV} / \mathrm{HOCl}$, $\mathrm{UV} / \mathrm{ClO}_{2}, \mathrm{UV} / \mathrm{H}_{2} \mathrm{O}_{2}$, and photo-Fenton, were found to be effective on removal of a broad 


\section{Chapter 1}

range of micropollutants [58, 71, 132-134, 137-140]. However, these techniques still have some drawbacks. For instance: use of ozone would lead to bromate production from bromide, which is a suspected human carcinogen [59]; use of chlorine, ozone, hydrogen peroxide requires facilities for dosing and storing these hazardous chemicals; the production of iron sludge is a major concern of the Fenton based processes, due to precipitation of $\mathrm{Fe}(\mathrm{OH})_{3}$, which calls for proper disposal/treatment of the sludge [85]; etc. Therefore, heterogeneous techniques, especially photocatalysis is considered to be a good approach for micropollutant removal because of its potential to overcome the abovementioned limitations and the ability to produce strong ROS, i.e. $\cdot \mathrm{OH}$.

Although many semiconductors have photocatalytic activity, $\mathrm{TiO}_{2}$ is the most used photocatalyst in environmental applications including air pollution control [101] and water treatment [102], because of its low toxicity, low cost, high efficiency, and high stability. Many research works at lab-scale have been carried out to removal micropollutants from water using suspension of $\mathrm{TiO}_{2}$ fine particles [61, 107, 141-143]. The use of suspension of $\mathrm{TiO}_{2}$ fine particles is beneficial for fundamental research at lab-scale but not favourable in real life implication. The use of $\mathrm{TiO}_{2}$ suspension requires solid/liquid separation process like ultrafiltration and centrifuge for better retention and reuse of the catalyst, resulting in additional costs. In addition, the suspended $\mathrm{TiO}_{2}$ particles result in increasing scattering of light, thus decreasing in the overall energetic efficiency. Previous study also shows the instability of suspended catalyst particles: the catalyst particles tend to form aggregates in water with increasing alkalinity [144]. Therefore, there is a need for immobilized $\mathrm{TiO}_{2}$ catalyst in real life implementations.

In recent decades, attempts have been made to employ immobilized $\mathrm{TiO}_{2}$ for elimination of organic pollutants from aqueous streams [105, 122, 145-148]. For instance, Murgolo et al. applied nanostructured $\mathrm{TiO}_{2}$ film deposited on a stainless steel mesh for photocatalytic degradation of a mixture of 10 micropollutants at $\mu \mathrm{g} / \mathrm{L}$ range under UV-C radiation [149]. Manassero et al. applied $\mathrm{TiO}_{2}$-coated glass rings for photocatalytic degradation of clofibric acid in a-fixed bed reactor [150]. Chiou et al. reported the application of $\mathrm{TiO}_{2}$ immobilized on glass beads for degradation of di-n-butyl phthalate [105]. Several methods for immobilization of $\mathrm{TiO}_{2}$ onto supports have been successfully employed 
in previous studies, e.g. sol-gel coating, spray coating, chemical vapor deposition, hydrothermal methods, and electrophoretic deposition, and a recent review paper on this topic is available [151]. A part from the above mentioned ones, the self-organized $\mathrm{TiO}_{2}$ nanotube array (TNA) produced by electrochemical anodization exhibits great potential. The TNA produced with this method has several advantages: (1) strong connection between the TNA layer and the support material (titanium) and thus high mechanical stability; (2) easily tunable morphology and crystalline phase; (3) easy to make.

The production of $\mathrm{TiO}_{2}$ nanotube arrays via electrochemical anodization method was firstly reported by Zwilling et al., [152, 153]. Their work showed that organized nanotube layers were formed after anodic oxidization of titanium substrate in chromic acid electrolytes containing hydrofluoric acid, and recognized that addition of fluoride ions to an electrolyte are the key to form these $\mathrm{TiO}_{2}$ nanotube structures. Since then, vast research efforts have been taken in this field [154-158]. At present, the mechanism of producing TNA by anodization has been well documented in literature [159]. Figure 1.5 illustrates the typical configuration of anodization TNA production process. The production of TNA by anodization method is usually carried out by applying a constant anodic voltage on the titanium in a fluoride containing electrolyte (usually 1-30 V in aqueous electrolytes or 5-150 $\mathrm{V}$ in organic electrolytes [159]). Initially, the titanium surface is oxidized to $\mathrm{TiO}_{2}$ under the applied anodic voltage (equation 1.4). Then, with the presence of fluoride ions, etching of the formed $\mathrm{TiO}_{2}$ layer and complexation of $\mathrm{Ti}^{4+}$ with fluoride ions will take place, resulting in formation of soluble $\left[\mathrm{TiF}_{6}\right]^{2-}$ (equation 1.5 and 1.6). A balance between these two processes leads to formation of the TNA. More detailed mechanistic theories are well described in a review by Schmuki et al. [159]. As-formed TNAs are typically present in amorphous form and have therefore low photocatalytic performance, so in order to obtain desired crystalline phases (usually anatase or rutile), annealing is needed after anodization. Typical annealing temperatures applied to obtain anatase are between $280{ }^{\circ} \mathrm{C}$ to $450{ }^{\circ} \mathrm{C}$, while higher annealing temperatures would lead to formation of rutile [160].

$$
\begin{aligned}
& \mathrm{Ti}+2 \mathrm{H}_{2} \mathrm{O} \rightarrow \mathrm{TiO}_{2}+4 \mathrm{H}^{+}+4 \mathrm{e}^{-} \\
& \mathrm{TiO}_{2}+6 \mathrm{~F}^{-} \rightarrow\left[\mathrm{TiF}_{6}\right]^{2-} \\
& \mathrm{Ti}^{4+}+6 \mathrm{~F}^{-} \rightarrow\left[\mathrm{TiF}_{6}\right]^{2-}
\end{aligned}
$$




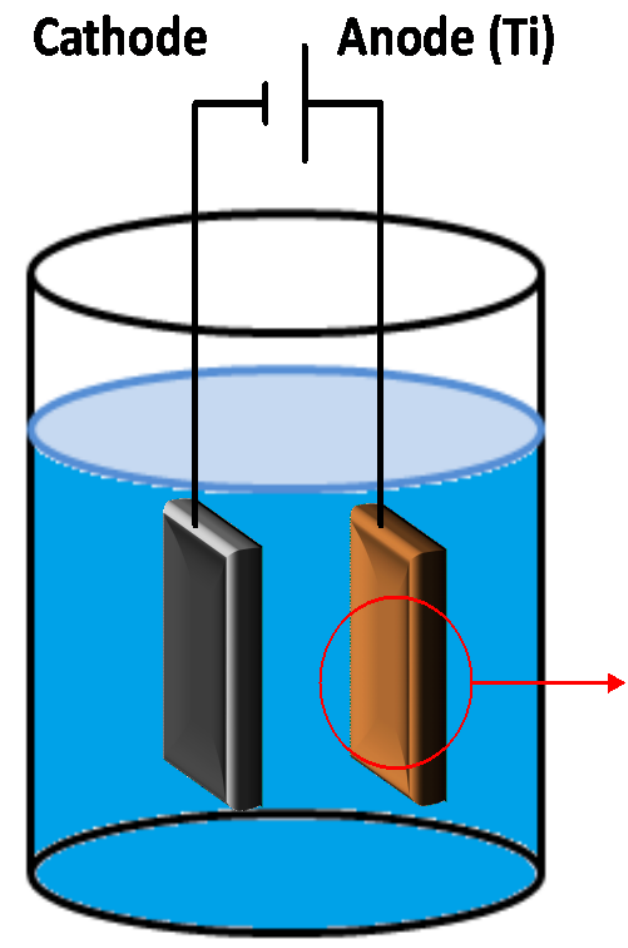

\section{$\mathrm{TiO}_{2}$ nanotubes}

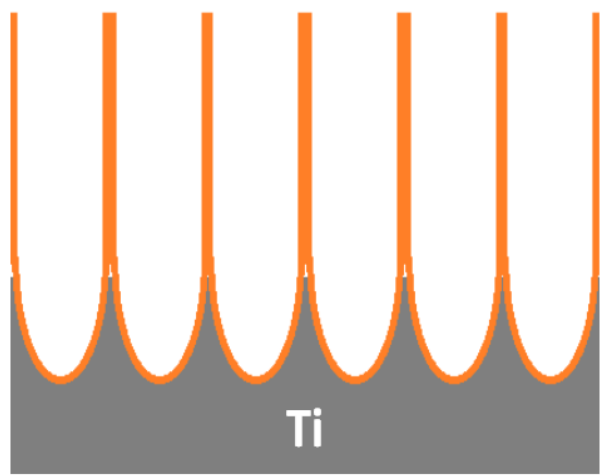

Figure 1. 5 The electrochemical anodization process for TNA production

Several operational parameters were reported to impose impact on the properties of the produced TNA. For instance, the applied voltage regulates the pore size of the TNA, the anodization time affects the thickness of the TNA layers, while the annealing conditions have effect on the crystalline composition $[159,160]$. By well regulating the operational parameters during anodization, tailored TNA with designated properties can be obtained.

The use of TNA for photocatalytic removal of organic pollutants has received attention, and examples are available in literature [161-164]. Very recently, attempts have been made to apply TNA for photocatalytic removal of micropollutants. For instance, Arfanis et al. reported application of TNAs for photocatalytic degradation of salicylic acid and caffeine, and demonstrated nearly $50 \%$ removal of both contaminants within the treatment time span, and good reusability of the TNAs [165]. 
However, studies on the application of TNA for micropollutants are still not abundant, and numerous knowledge gaps still exist:

* As aforementioned, the morphology and crystalline phase of TNA are easily tuneable. These properties may impose effects on the photocatalytic micropollutant removal processes: for instance, the morphology of TNA layer may affects the adsorption/desorption of both the target pollutant and photoinduced ROS on the TNA surface. However, little is known about the effects of TNA properties on photocatalytic micropollutant removal.

* Regarding the real life application, various background water constituents have been reported to impose detrimental effects on the $\mathrm{TiO}_{2}$ slurry based photocatalytic systems [106, 166-168]. Due to the morphological differences between $\mathrm{TiO}_{2}$ fine particles and TNA, the interaction between background water constituents with TNA may differ from that in the case of $\mathrm{TiO}_{2}$ fine particles based system. However, little is known about how the water quality parameters would affect the performance of TNA based systems.

* Little is known about the contribution of different reaction sites (e.g. surface reaction, reaction in liquid phase) in the TNA based system.

Another major drawback of $\mathrm{TiO}_{2}$ photocatalytic processes is the fast recombination of $\mathrm{e}^{-} / \mathrm{h}^{+}$pairs, where a large amount of energy from absorbed photons is lost as heat, resulting in seriously reduced treatment efficiency [124]. Therefore, reducing the recombination of $\mathrm{e}^{-} / \mathrm{h}^{+}$pairs is a key to obtain effective photocatalytic process. Efforts have been made on this aspect, e.g. integrating $\mathrm{TiO}_{2}$ with other active materials to form in-situ hetero-junction for better $\mathrm{e}^{-} / \mathrm{h}^{+}$separation $[169,170]$, applying voltage bias - generally known as photoelectrocatalysis (PEC) - to separate $\mathrm{e}^{-} / \mathrm{h}^{+}$from the catalyst $[124,125]$. Recently a novel PEC technique, known as photocatalytic fuel cell (PFC) has been introduced into the field of water treatment $[171,172]$. In PFC systems, the photo-induced electrons can migrate from $\mathrm{TiO}_{2}$ to the cathode through an external connection, and driven by the photon-induced self-bias between the two electrodes, without external power supply. Hereby the photo-induced holes are left at the $\mathrm{TiO}_{2}$ surface triggering degradation of the target pollutants $[171,173]$. In this way, the recombination of $\mathrm{e}^{-} / \mathrm{h}^{+}$pairs is reduced and 


\section{Chapter 1}

consequently enhanced target contaminants removal. Compared to conventional PEC processes, the PFC systems do not require electrical power supply for separation of $\mathrm{e}^{-} / \mathrm{h}^{+}$, therefore the PFC concept is gaining increasing attention in the field of water treatment in recent years. For example, enhanced organic pollutant degradation and simultaneous electricity generation was reported in a study conducted by $\mathrm{Li}$ et al., using a $\mathrm{TiO}_{2} / \mathrm{Ti}-\mathrm{Pt} \mathrm{PFC}$ treating Reactive Brilliant Red X-3B [173]. However, not many attempts have been made to apply PFC for micropollutant removal and numerous knowledge gaps still exist:

\footnotetext{
* Little is known about the efficacy of PFC on micropollutant removal from water.

* Little is known on micropollutant degradation mechanisms in PFC systems.

* Little is known about the effects of water quality parameters on the performance of PFC systems.

* Little is known about the applicability of PFC systems for micropollutant removal in complex water matrices like real wastewater effluents.
}

Therefore, research aims at the above described knowledge gaps would contribute to both the further development and a better understanding of $\mathrm{TiO}_{2}$ photocatalytic techniques for micropollutant removal, and provide new perspectives in the research field on photochemical micropollutant removal techniques.

\subsubsection{Call for alternative photochemical technique}

The primary ROS in most photochemical micropollutant removal techniques, e.g. $\mathrm{UV} / \mathrm{Cl}_{2}, \mathrm{UV} / \mathrm{O}_{3}, \mathrm{UV} / \mathrm{HOCl}, \mathrm{UV} / \mathrm{ClO}_{2}, \mathrm{UV} / \mathrm{H}_{2} \mathrm{O}_{2}$, photo-Fenton, and $\mathrm{UV} / \mathrm{TiO}_{2}$ are strong radicals like $\cdot \mathrm{OH}$ and $\cdot \mathrm{Cl}$. The strong oxidizing power of these strong radicals enabled the applications of such techniques for various purposes, including organic pollutant removal, disinfection etc. However, application of these techniques is usually limited as a polishing process for relatively clean water. It is well documented that, in relatively complex water matrixes, the water matrices, including inorganic salts (e.g. phosphate, bicarbonate, sulphate) and natural organic matters (NOMs), impose large detrimental effect on the performance of such techniques [166]. For instance, Autin et al. evaluated the impact of background organic matter and alkalinity on the degradation of the pesticide metaldehyde in $\mathrm{UV} / \mathrm{TiO}_{2}$ and $\mathrm{UV} / \mathrm{H}_{2} \mathrm{O}_{2}$ processes [174]. Their results show that: (1) both the background organic matter 
and alkalinity act as $\cdot \mathrm{OH}$ scavengers in the $\mathrm{UV} / \mathrm{H}_{2} \mathrm{O}_{2}$ process which adversely affect micropollutants degradation; (2) the inhibitory effect of alkalinity in $\mathrm{UV} / \mathrm{TiO}_{2}$ photocatalysis is much more pronounced due to the formation of large $\mathrm{TiO}_{2}$ aggregates; (3) UV/ $\mathrm{TiO}_{2}$ and $\mathrm{UV} / \mathrm{H}_{2} \mathrm{O}_{2}$ processes are controlled by the background scavenging rate regardless of the concentration of micropollutant. Same inhibitory effect of background organic matter and alkalinity was confirmed and further studied in a subsequent study by the same authors [175]. Carra et al. compared performance of different UV AOPs for acetamiprid removal in different water matrices, and reported the negative effect of humic acids on the examined UV AOPs [176]. In a study applying UV/Cl 2 for atrazine degradation, it is found that the degradation efficiency was greatly inhibited by NOM [134]. In photocatalytic processes, anions like phosphate and bicarbonate remain strongly adsorbed onto catalysts surfaces and can therefore deactivate the site where it adsorbed $[96,166]$. Furthermore, inorganic anions like phosphate and bicarbonate are also able to act as hydroxyl radical scavengers. A study conducted by J. Brame et al. [177] reported that the presence of phosphate ions even at low concentrations could cause significant decrease in the performance of $U V / \mathrm{H}_{2} \mathrm{O}_{2}$ process, which was attributed to scavenging of hydroxyl radicals by phosphate ions. NOMs interfere with photochemical micropollutant removal techniques by two main mechanisms. The first one is absorption of UV light, which is well known as "inner filter effect". NOMs are usually able to absorb light in the UV and near-UV range. The presence of NOMs in water would decrease the availability of UV light for semiconductors $/ \mathrm{H}_{2} \mathrm{O}_{2}$ and any other species needed to produce hydroxyl radicals, in the cases of all UV related AOPs. The other reason that NOM could reduce the performance of most existing AOPs is that NOMs can act as hydroxyl radical scavenger.

In order to be able to remove micropollutants from complex water matrices, studies have been carried out to investigate the efficacy of employing photosensitization processes for organic chemical removal in various water matrices [64, 129, 130], where singlet oxygen $\left({ }^{1} \mathrm{O}_{2}\right)$ was considered to be the primary oxidant. As an oxidant which has a higher oxidizing potential (about $1 \mathrm{~V}$ higher [127]) than ground state oxygen and electrophility, it selectively reacts with organics containing electron-rich functional groups, as described in section 1.2. Because generally only a very small fraction of NOM contains electron-rich moieties [177], the scavenging effect of NOM may be minimal. Its application 


\section{Chapter 1}

in organic pollutant elimination has been investigated and the ability to overcome detrimental effects of background water constituents has been documented [178-181]. Although in most previous studies solid photosensitizers were applied [129, 130], involvement of solid photosensitizers still has drawbacks. Because ${ }^{1} \mathrm{O}_{2}$ has a very short life time, the presence of ${ }^{1} \mathrm{O}_{2}$ is limited to a thin layer close to the solid phase; therefore only adsorbed pollutants can be well degraded [182]. Moreover, inorganic ions and NOMs in water matrices can still reduce the process efficiency by blocking the active site of solid sensitizers. In this manner, homogeneous singlet oxygen mediated processes can be an alternative, because the aforementioned problems associated with heterogeneous system can be avoided. Methylene blue (MB) has great potential to be utilized as homogeneous photosensitizer for such purpose. It has strong light absorption in visible range (red light) which can avoid the inner filter effect of NOMs in complex water matrices, has a relatively high quantum yield of singlet oxygen formation [183], and has a long history of safe use in human treatment without major side effect. The red light induced MB photosensitization process for singlet oxygen formation is reported to have a broad application field including disinfection in not only wastewater treatment but also blood products [184-189], where singlet oxygen was generated to kill pathogens, as well as medical therapy, where red light induced MB photosensitized singlet oxygen was directed to selectively kill tumour cells for cancer therapy [187]. Nevertheless, no attempt has yet been made to apply the MB photosensitization process for micropollutant removal and numerous research opportunities exist:

\footnotetext{
* Is it possible to achieve micropollutant removal by MB photosensitization?

* What are the degradation mechanisms contributing to micropollutant removal in the MB photosensitization process?

* What are the effects of background water constituents?

* What are the key operational parameters and their effects?
}

\subsection{Scope and outline of this thesis}

The general objective of this thesis is to contribute to a better understanding and further development of photochemical treatment processes for micropollutant removal from aqueous streams. Referring to the above described research opportunities and 
knowledge gaps, emphasis of this thesis is given to two topics: (1) Development of an alternative photochemical water treatment technique for micropollutant removal from wastewater; (2) Further development of $\mathrm{UV} / \mathrm{TiO}_{2}$ technique by application of TNA as immobilized catalyst.

Chapter 2 contributes to a better understanding on application of TNA for photocatalytic micropollutant removal techniques. It investigates fabrication of TNA and evaluates its use as immobilized photocatalyst for micropollutant degradation in water.

Chapter 3 contributes to a better understanding of applicability of TNA as photocatalyst in complex water matrices. Emphasis was given to the potential detrimental effect of dissolved natural organic matter (NOMs) on micropollutant removal in TNA based photocatalytic system.

Chapter 4 contributes to a better understanding on how to further improve the TNA based photocatalytic micropollutant removal process. It presents that the membranefree TNA based photocatalytic fuel cell system can successfully enhance micropollutant removal, compared to conventional TNA based photocatalytic system. Mechanisms of the enhancement and effects of $\mathrm{pH}$, electrolyte and water matrices are here being discussed.

Chapter 5 contributes to development of alternative photochemical water treatment technique. It presents the proof of principle of applying red light induced methylene blue photosensitization (MB/Red-light) for micropollutant removal. The efficacy of $\mathrm{MB} /$ Red-light on degradation of four pharmaceutical compounds was investigated. The primary ROS in applying this technique was identified. Effects of $\mathrm{pH}$ and commonly present water constituents were studied.

Chapter 6 contributes to a better understanding on how to optimize the proposed $\mathrm{MB} /$ Red-light technique. It reports the effect of operational parameters on the performance of $\mathrm{MB} /$ Red-light technique. Effects of MB dosage, initial micropollutant concentration, and aeration rate were studied.

In Chapter 7 findings and outcomes from previous chapters were integrated and reflected. Moreover, new perspectives on future research opportunities are provided. 


\section{Chapter 2}

\section{Photocatalytic degradation of metoprolol by}

\section{$\mathrm{TiO}_{2}$ nanotube arrays and UV-LED: effects of}

catalyst properties, operational parameters,

commonly present water constituents, and

photo-induced reactive species

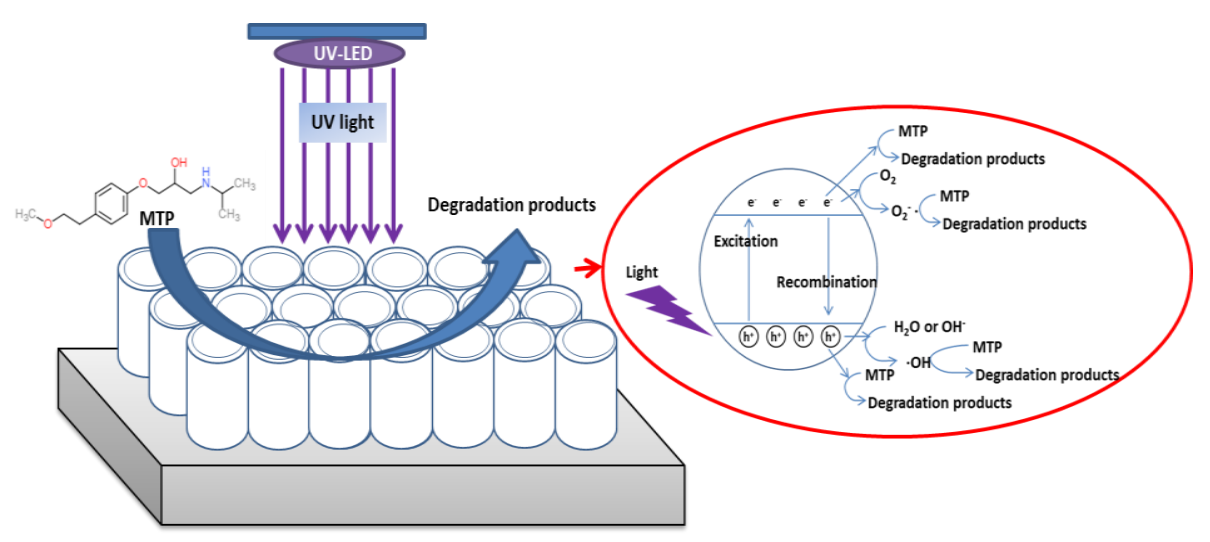

This Chapter has been published as:

Y. Ye, Y. Feng, H. Bruning, D. Yntema, H. Rijnaarts, Photocatalytic degradation of metoprolol by $\mathrm{TiO}_{2}$ nanotube arrays and UV-LED: effects of catalyst properties, operational parameters, commonly present water constituents, and photo-induced reactive species, Applied Catalysis B: Environmental 220 (2018) pp. 171-181 


\section{Chapter 2}

\section{Abstract}

The aim of this study was to evaluate the use of self-organized TiO2 nanotube arrays (TNAs) as immobilized catalyst and UV-LED as light source (UV-LED/TNAs) for photocatalytic degradation of the $\beta$-blocker metoprolol (MTP) from aqueous solution. Firstly we employed electrochemical anodization to synthesize self-organized TNAs, and the effect of anodization potential and annealing temperature was examined. Characterization by SEM demonstrated a linear relation between the diameter of $\mathrm{TiO}_{2}$ nanotubes produced and the anodization potential, while Raman measurement revealed the vital role of annealing on crystallographic composition of the anodic produced $\mathrm{TiO}_{2}$ nanotubes. Regarding their performance in photocatalytic MTP degradation, surface morphology and crystallographic composition of the TNAs were found to impose crucial influence: only TNAs with diameter not smaller than $53 \mathrm{~nm}$ enabled rapid MTP degradation, and highest MTP degradation was obtained when a mixture of anatase and rutile were present in the TNAs. Secondly, the effect of operational parameters, i.e initial MTP concentration, $\mathrm{pH}$, was investigated. Initial MTP concentration at low level had no detrimental effect on the process performance. Rapid MTP degradation and high total removal were achieved in a wide $\mathrm{pH}$ range (3-11). To evaluate the applicability of TNAs for water treatment, experiments were first carried out in the presence of three different commonly present water constituents, i.e bicarbonate ions, phosphate ions, and natural organic matters (NOMs). The results show that bicarbonate and phosphate ions have no inhibitory effect at concentration levels up to $200 \mathrm{mg} / \mathrm{L}$, and NOMs exhibit detrimental effect when their concentration exceeds $5 \mathrm{mg} / \mathrm{L}$. The total removal MTP degradation reduced from $87.09 \%$ to $62.05 \%$ when tap water samples were applied, demonstrating reasonable efficacy for practical applications. Regarding the degradation mechanism, formic acid and tert-butanol were added as scavenger for photo-generated holes $\left(\mathrm{h}^{+}\right)$and hydroxyl radicals $(\cdot \mathrm{OH})$, respectively. The obtained results demonstrate that primary degradation process occurred in liquid phase with participation of hydroxyl radicals in the liquid phase $(\cdot \mathrm{OH}$ liquid), while smaller portion of MTP were degraded on the catalysis surface via reaction with $\mathrm{h}^{+}$and hydroxyl radicals adsorbed on the catalyst surface $(\cdot \mathrm{OH}$ surface). Other reactive species, e.g photo generated electrons and superoxide radical anions, did also play a minor role in MTP degradation. The 
Chapter 2

mechanistic aspect was further confirmed by identification of degradation products by LCMS/MS. The TNAs exhibited good stability after repeated use under varied operation conditions. 


\section{Chapter 2}

\subsection{Introduction}

In recent years, a large number of pharmaceuticals have been detected at various concentration levels in the aqueous environment $[7,27]$, from ground and surface water to even drinking water resources $[5-11,190]$. This issue is gaining concerns because of the potential risks for aquatic life and human society. On the other hand, the presence of such pollutants in various water bodies together with treatability studies clearly demonstrate the poor efficacy of conventional wastewater treatment processes and drinking water production processes on removal of wide range of pharmaceutical compounds [64, 102, 190]. Among the wide range of pharmaceutical compounds frequently detected in the environment, a $\beta$-blocker, i.e metoprolol (MTP), is considered to be an emerging contaminant: (1) it has been classified in the group of pharmaceuticals most commonly present in the environment [7]; (2) it is widely used in both hospitals and households, and enters surface waters (including drinking water sources) at up to $\mu \mathrm{g} / \mathrm{L}$ level [103]; (3) environmental risk assessment studies have indicated environmental risks associated with its presence[26, 191]. Therefore, removal of such compounds from aqueous streams, especially during drinking water production, has become an urgent topic, to guard the safety of water supply and improve the quality of life.

Advanced oxidation processes (AOPs), where a strong oxidizing species hydroxyl radical $(\cdot \mathrm{OH})$ is the primary oxidant, exhibit sufficient efficacy in elimination of a wide range of organic contaminants including pharmaceuticals [55, 83, 192], among which photocatalysis is considered to be a good approach because of its chemical-free nature. Although many semiconductors have photocatalytic activity, $\mathrm{TiO}_{2}$ is the most used photocatalyst in environmental applications including air pollution control [101] and water treatment [102], because of its low toxicity, low cost, high efficiency, and high stability. Principles of $\mathrm{TiO}_{2}$ photocatalytic processes have been described elsewhere $[62,103]$. The photocatalytic degradation of organic pollutants is usually initiated by excitation of $\mathrm{TiO}_{2}$ by photons containing energy higher than the $\mathrm{TiO}_{2}$ band gap. Sequentially, electron/hole $\left(\mathrm{e}^{-}\right.$ $/ h^{+}$) pairs are generated on the catalyst surface. The photo-generated electrons and holes are able to participate in direct redox reactions with target organic contaminants. Besides, the photo-generated electrons and holes can also react with oxygen, water, or $\mathrm{HO}^{-}$to 
generate highly reactive species, e.g hydroxyl radicals ( $\mathrm{HO} \cdot)$, superoxide radical anions $\left(\mathrm{O}_{2} \cdot{ }^{-}\right)$, etc, and those in-situ generated reactive species are able to oxidize and reduce many contaminants. Applicability of $\mathrm{TiO}_{2}$ for elimination of a wide range of organic contaminant has been well documented [105-109].

Recently, regarding real life application, attempts have been made to employ immobilized catalyst for elimination of organic pollutants from aqueous streams [105, 122, 145-148], in order to achieve better retention and reuse of the catalyst. Among all kinds of immobilized $\mathrm{TiO}_{2}$, the self-organized $\mathrm{TiO}_{2}$ nanotube arrays (TNAs) exhibit great potential because of its relatively large surface area, high stability, and oriented electron transport as well as high electron mobility which could reduce electron/holes $\left(\mathrm{e}^{-} / \mathrm{h}^{+}\right)$recombination in the material $[193,194]$. Although there are various approaches for $\mathrm{TiO}_{2}$ nanotube arrays synthesis, electrochemical anodization has gained the most attention because it is a fast and facile method [161], and easy to tune the morphology of $\mathrm{TiO}_{2}$ nanotubes produced. Synthesis of $\mathrm{TiO}_{2}$ nanotube arrays via anodization of Ti material has been previously studied by other researchers [152, 153, 193, 195]. Among various factors exerting impact on the characteristics of anodic produced $\mathrm{TiO}_{2}$ nanotube arrays, anodization potential and annealing temperature are two vital parameters which control the diameter and crystallographic structure of the $\mathrm{TiO}_{2}$ nanotube arrays, respectively $[159,160]$. Furthermore, the diameter and crystallographic structure are reported to impose impact on photocatalytic performance of the $\mathrm{TiO}_{2}$ nanotube arrays $[162,196]$. The use of $\mathrm{TiO}_{2}$ nanotube arrays for photocatalytic removal of organic pollutants has received attention, and examples are available in literature [161-164].

Regarding implications of photocatalysis, light source is another vital factor because it imposes significant impact on the operational and maintenance costs. Mercury lamps have been widely used for photocatalytic pollutants elimination processes, which are reported to have major drawbacks: (1) short working life span (500-2000h); (2) hazardous materials (mercury) content; (3) fragility [197]. Recent advances in light emitting diode (LED) technology provides better alternative, due to their advantages: short warm-up time, no hazardous materials content, long working life span, compatibility, narrow light emission spectra, etc [197]. Therefore, in recent years the use of UV-LED for photocatalytic treatment 


\section{Chapter 2}

of various organic contaminants is gaining research interests [198-200]. Only very few attempts have been made to combine the use of TNAs and UV-LED, for dye degradation [164].

To the best of the authors' knowledge, no research has been conducted on the photocatalytic treatment of pharmaceutical compounds using self-organized TNAs as catalyst and UV-LED as light source. In this context, in the present study we aimed to evaluate the performance of the combination of self-organized TNAs as immobilized catalyst and UV-LED as light source (UV-LED/TNAs) for photocatalytic degradation of the $\beta$ blocker MTP from aqueous solution. Synthesis and characterization of the self-organized $\mathrm{TiO}_{2}$ nanotube arrays was studied, and the effect of two operational parameters, i.e. anodization potential and annealing temperature, on the properties of $\mathrm{TiO}_{2}$ nanotube arrays was examined. Moreover, the impact of $\mathrm{TiO}_{2}$ nanotube arrays ' characteristics on their photocatalytic performance was studied. Regarding photocatalytic degradation of MTP over $\mathrm{TiO}_{2}$ nanotube arrays, the effect of multiple operational parameters and the effect of back ground water constituents were investigated. Additionally, its applicability in drinking water was investigated by conducting experiments in tap water samples. Furthermore, experiments with addition of specific scavengers were carried out to understand the mechanistic aspect, and contributions of the different reactive species and reaction mechanism were identified. The stability of TNAs in the UV-LED/TNAs photocatalytic system was also evaluated.

\subsection{Materials and methods}

\subsubsection{Materials}

Titanium foil ( $\geq 99.5 \%, 0.3 \mathrm{~mm}$ thick) was purchased from the Titaniumshop (The Netherlands). Ammonium sulphate ( $\geq 99.0 \%)$, ammonium fluoride $(\geq 98.0 \%)$, tert-Butanol $(\geq 99.0 \%)$, formic acid ( $\geq 96 \%$ ) were obtained from Sigma-Aldrich (Germany). Metoprolol tartrate salt ( $\geq 98.0 \%)$ was purchased from Sigma-Aldrich (Germany) and was used as received. Stock solution of metoprolol tartrate salt was prepared at MTP concentration of $200 \mathrm{mg} / \mathrm{L}$. Acetone ( $\geq 99.7$ ), 2-propanol ( $\geq 99.9 \%$ ), sodium carbonate ( $\geq 99.9 \%)$, hydrochloric acid $(0.1 \mathrm{~mol} / \mathrm{L})$, tri-sodium phosphate dodecahydrate $(\geq 98.0 \%)$, and sodium hydroxide 
aqueous solution (1 mol/L) were purchased from VWR (Belgium) and was used as received. An aquatic NOM Suwannee River NOM (2R101N) was obtained from International Humic Substances Society (IHSS) and received as dry solid extracts. Stock solution of the Suwannee River NOM was prepared at concentration of $100 \mathrm{mg} / \mathrm{L}$. Ultrapure water from a Milli-Q Advantage A10 system (Merck Millipore, Darmstadt, Germany) was used for preparation of stock solutions and reaction solutions.

\subsubsection{Synthesis of $\mathrm{TiO}_{2}$ nanotube arrays by electrochemical anodization}

As pretreatment, the Ti foils were first degreased by successively ultra-sonication for 15 min in 2-propanol, acetone and Milli-Q water, and then were dried in $\mathrm{N}_{2}$ atmosphere. The $\mathrm{TiO}_{2}$ nanotube arrays (TNAs) were synthesized by electrochemical anodization in a twoelectrode chemical cell connected to an EST150 DC power supply (Delta Elektronika, The Netherlands). A Ti foil with a size of $3 \mathrm{~cm} \times 4.5 \mathrm{~cm}$ was used as anode, and a stainless steel foil with a size of $4.5 \mathrm{~cm} \times 5 \mathrm{~cm}$ was used as cathode. The distance between the electrodes was $2 \mathrm{~cm}$. The Ti foil was anodized in $100 \mathrm{~mL}$ aqueous electrolyte $\left(0.15 \mathrm{~mol} / \mathrm{L} \mathrm{NH}_{4} \mathrm{~F}+1 \mathrm{~mol} / \mathrm{L}\right.$ $\left.\left(\mathrm{NH}_{4}\right)_{2} \mathrm{SO}_{4}\right)$ [125] under designated voltage for 2 hours. Then the obtained foil was first rinsed and then further sonication washed with Milli-Q water to remove residue electrolyte and impurities on the surface. Subsequently, the treated Ti foil was annealed at designated temperatures for $0.5 \mathrm{~h}$ in a furnace (Nabertherm, Germany) in air atmosphere and was cooled gradually back to ambient temperature after annealing. The designated anodization potentials and corresponding annealing temperatures for synthesis of different TNAs are summarized herein in Table 2. 1. 


\section{Chapter 2}

Table 2. 1 Anodization potentials and annealing temperatures for different TNAs samples

\begin{tabular}{ccc}
\hline Sample & Anodization potential (V) & Annealing temperature $\left({ }^{\circ} \mathrm{C}\right)$ \\
\hline 1 & 1 & 450 \\
2 & 5 & 450 \\
3 & 10 & 450 \\
4 & 15 & 450 \\
5 & 20 & 450 \\
6 & 25 & 450 \\
7 & 20 & No annealing \\
8 & 20 & 300 \\
9 & 20 & 600 \\
\hline
\end{tabular}

\subsubsection{Characterization of $\mathrm{TiO}_{2}$ nanotube arrays}

The surface morphologies of the prepared TNAs were characterized by scanning electron microscopy (SEM) and the chemical composition of the prepared TNAs was measured by EDX. The SEM/EDX analyses were performed using a JEOL JSM 6480 scanning electron microscope (JEOL Europe B.V., Nieuw-Vennep, The Netherlands) equipped with a EDX system. Surface area measurements of TNAs samples prepared under different anodization potentials were performed using a Tristar 3000 Surface area and Porosity Analyzer (Micromeritics, USA), where nitrogen adsorption isotherms were collected at $77 \mathrm{~K}$ $\left(-196{ }^{\circ} \mathrm{C}\right)$ and the Brunauer-Emmertt-Teller model was used to determine the specific surface area. Raman spectra of the prepared TNAs samples were recorded using a Horiba LabRAM spectrometer equipped with a mpc3000 laser (532.2 nm), an $800 \mathrm{~mm}$ focal length achromatic flat field monochromator (grating of 600 grooves $/ \mathrm{mm}$ ) and a Synapse multichannel air cooled $\left(-70^{\circ} \mathrm{C}\right) \mathrm{CCD}$ detector. 


\subsubsection{Photocatalytic degradation of metoprolol}

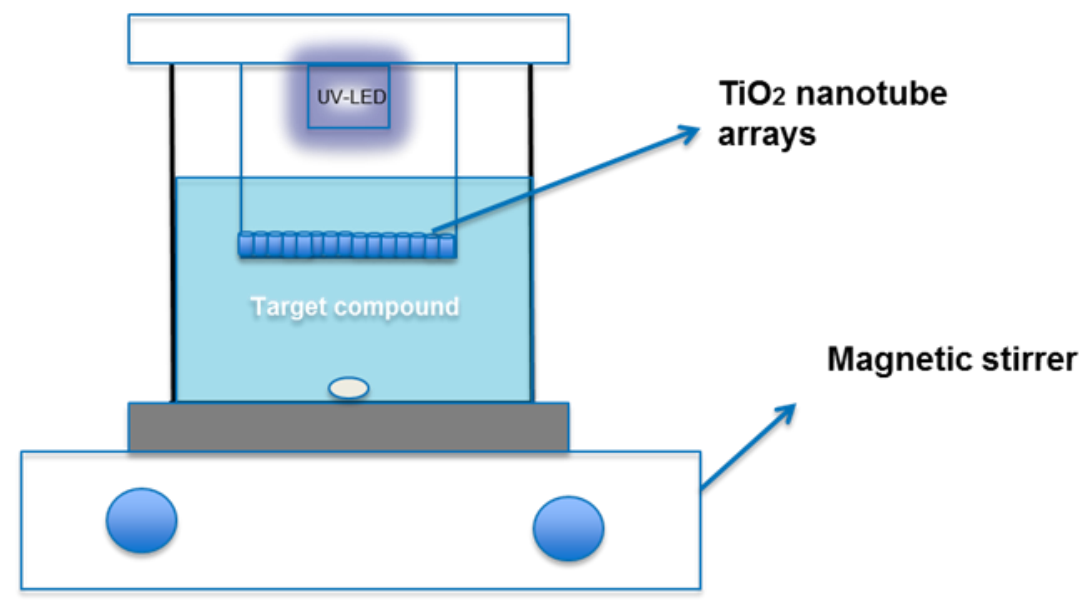

Figure 2. 1 Schematic of the experimental set-up

The photocatalytic experiments were conducted in a Petri dish with a $450 \mathrm{~mW}$ UVLED light source module placed on the top. The UV-LED light source module consisted of a UV-LED (NCSU033B, NICHIA, Japan) and has peak emission wavelength of $365 \mathrm{~nm}$, and an aluminum plate served as heat dispenser. A schematic of the set-up is shown in Figure 2. 1. The experiments were carried out with a reaction solution volume of $60 \mathrm{~mL}$, with presence of desired TNAs (size of $3 \mathrm{~cm} \times 3 \mathrm{~cm}$ ), at ambient temperature. The distance between the UV-LED and the surface of TNAs was $2 \mathrm{~cm}$. Continuous mixing started as soon as the reaction solution was added into the Petri dish. Before switching on the UV-LED, the reaction system was kept in dark for 1 hour to establish saturation of any possible adsorption of MTP on TNAs surface. Then the photocatalytic experiments started, for duration of $120 \mathrm{~min}$. The radiant power of the UV-LED reached the surface of TNAs was $18.6 \mathrm{~mW} / \mathrm{cm}^{2}$, measured by a THORLABS S150C radiant power meter (THORLABS, USA). $1 \mathrm{~mL}$ samples were taken at designated time intervals, and stored in dark at $4{ }^{\circ} \mathrm{C}$ till LC-MS/MS analysis. All experiments were carried out in duplicate. Except for experiments conducted in designated acid or alkaline conditions, all other experiments were conducted with natural initial pH (circumneutral: 6-7) and no $\mathrm{pH}$ adjustment was applied. 
Table 2. 2 Characteristics of the tap water

\begin{tabular}{ccc}
\hline Parameters & Unit & Values \\
\hline $\mathrm{Cl}$ & $\mathrm{mg} / \mathrm{l}$ & 60.1 \\
$\mathrm{NO}_{2}{ }^{-}$ & $\mathrm{mg} / \mathrm{l}$ & $<0.05$ \\
$\mathrm{NO}_{3}{ }^{-}$ & $\mathrm{mg} / \mathrm{l}$ & 8.84 \\
$\mathrm{PO}_{4}{ }^{3-}$ & $\mathrm{mg} / \mathrm{l}$ & $<0.05$ \\
$\mathrm{SO}_{4}{ }^{2-}$ & $\mathrm{mg} / \mathrm{l}$ & 1.75 \\
$\mathrm{TC}^{-}$ & $\mathrm{mg} / \mathrm{l}$ & 53.6 \\
$\mathrm{NPOC}^{-}$ & $\mathrm{mg} / \mathrm{l}$ & 4.26 \\
$\mathrm{IC}^{2+}$ & $\mathrm{mg} / \mathrm{l}$ & 49.3 \\
$\mathrm{Ca}^{2+}$ & $\mu \mathrm{g} / \mathrm{l}$ & 38100 \\
$\mathrm{Cu}^{2+}$ & $\mu \mathrm{g} / \mathrm{l}$ & 108 \\
$\mathrm{~K}^{+}$ & $\mu \mathrm{g} / \mathrm{l}$ & 2360 \\
$\mathrm{Mg}^{2+}$ & $\mu \mathrm{g} / \mathrm{l}$ & 9360 \\
$\mathrm{Na}^{+}$ & $\mu \mathrm{g} / \mathrm{l}$ & 66600 \\
\hline
\end{tabular}

For experiments regarding effect of initial MTP concentration, different initial MTP concentrations ranging from $0.1 \mathrm{mg} / \mathrm{L}$ to $1.5 \mathrm{mg} / \mathrm{L}$ were applied, while the initial MTP concentration in all other experiments were set to be $1.0 \mathrm{mg} / \mathrm{L}$. For experiments regarding the effect of background water constituents, NOMs, bicarbonate ions, or phosphate ions were added separately to the reaction solutions to designated concentration levels, and $\mathrm{pH}$ was adjusted using $\mathrm{HCl}$ standard solution to circum-neutral (6-7). For experiments regarding performance in tap water, real tap water (characteristics of the tap water are summarized in Table 2. 2) was used to prepare the reaction solution, while all other experiments were conducted in Milli-Q water. For experiments regarding the effect of intial $\mathrm{pH}, \mathrm{HCl}$ or $\mathrm{NaOH}$ standard solutions were used to adjust the initial $\mathrm{pH}$ of the reaction solutions to designated 
value. For mechanistic study, certain amount of specific scavengers (tert-butanol for $\cdot \mathrm{OH}$, and formic acid for $\mathrm{h}^{+}$) were added separately in accordance with a previous study [104].

It should be noticed that MTP does not absorb photons with wavelength longer than $290 \mathrm{~nm}$ (Figure S2. 2), so no direct photolysis of MTP could take place in our system.

\subsubsection{Analytical procedures}

For MTP concentrations and degradation by-products measurement, an Agilent LCMS/MS system consisting of Agilent infinity 1260 LC-system (degasser, binary pump, auto sampler with cooled tray and column oven) and Agilent 6420 triple Quadrupole Mass Spectrometer with Electrospray ion source was used. More detailed information of the analytical method used can be found in our previous study [65].

\subsection{Results and discussion}

\subsubsection{Synthesis of $\mathrm{TiO}_{2}$ nanotube arrays}

Synthesis of $\mathrm{TiO}_{2}$ nanotubes (TNT) arrays via anodization of $\mathrm{Ti}$ material has been previously studied by other researchers [152, 153, 193, 195]. Among various factors exerting impact on the characteristics of anodic produced TNAs, anodization potential and annealing temperature are two vital parameters which control the diameter of the TNTs and crystallographic structure of the $\mathrm{TiO}_{2}$, respectively $[159,160]$. It should be noticed that those impacts may vary among different systems. Therefore, experiments were performed to examine the effect of anodization potential and annealing temperature on the characteristics of $\mathrm{TiO}_{2}$ nanotube arrays in our applied system.

\subsubsection{Effect of anodization potential}

The results (Figure 2. 2 and Figure 2. 3) show that anodization potential imposes effects on two aspects of the anodized Ti foil surface. Firstly, a certain threshold anodization potential is needed to achieve total oxidation of the Ti foil surface to $\mathrm{TiO}_{2}$. As demonstrated in Figure 2. 3, complete oxidation, revealing the formation of $\mathrm{TiO}_{2}$, was only achieved in samples anodized under potential higher than $10 \mathrm{~V}$, while the atomic percentage of Ti was still rather high in samples anodized under $1 \mathrm{~V}$ and $5 \mathrm{~V}$. In addition, a significant amount of 


\section{Chapter 2}

F (approx. 2 atomic \%) entered the TNTs structures, which is in line with other work using fluoride containing electrolytes [160].

As shown in Figure 2. 2, anodization potential exerts vital impact on diameters of the produce TNAs. Self-organized TNAs structure were formed when applied anodization potential was higher than $10 \mathrm{~V}$, while no tubular nanostructure was obtained on samples anodized under $1 \mathrm{~V}$ and $5 \mathrm{~V}$, revealing that threshold anodization potential exists to trigger the growth of TNAs. In those samples with formation of TNAs, anodization potential exerts large effect on the average diameters of formed TNTs, and a clear anodization potential dependence of the TNAs diameter was observed: the average diameter of $\mathrm{TiO}_{2}$ nanotubes increased from $26 \mathrm{~nm}$ to $106 \mathrm{~nm}$ with increased anodization potential from $10 \mathrm{~V}$ to $25 \mathrm{~V}$ (Figure 2. 4). This is in accordance with previous studies. Yasuda, K. and P. Schmuki studied the parameters to control morphology and composition of anodic formed self-organized zirconium titanate TNAs, and found a linear correlation between the applied anodization potential and the diameter of TNAs in the range between $1 \mathrm{~V}$ and $100 \mathrm{~V}$ in electrolytes containing ammonium sulphate and ammonium fluoride [201]. Another study conducted by Macak et al. has reported that $\mathrm{TiO}_{2}$ nanotubes diameter is linearly dependent by the anodization potential (from $2 \mathrm{~V}$ to $40 \mathrm{~V}$ ) in glycerol/water/ammonium fluoride electrolytes [202]. The slope of the dependencies are different, which could be attributed to the different conductivity of electrolytes used in different studies which imposes large impact on the effective potential on the electrodes [159]. This linear correlation between TNTs diameter and the applied anodization voltage has significant potential for expanding application of TNAs, because based on which tailored TNAs with specific diameters could be produced. Moreover, specific surface area of TNAs was also affected by anodization potential, as shown in Figure 2. 2. The specific surface area of TNAs increased with increasing anodization potential from $10 \mathrm{~V}$ to $20 \mathrm{~V}$, while further increased anodization potential resulted in a decreased specific surface area. 


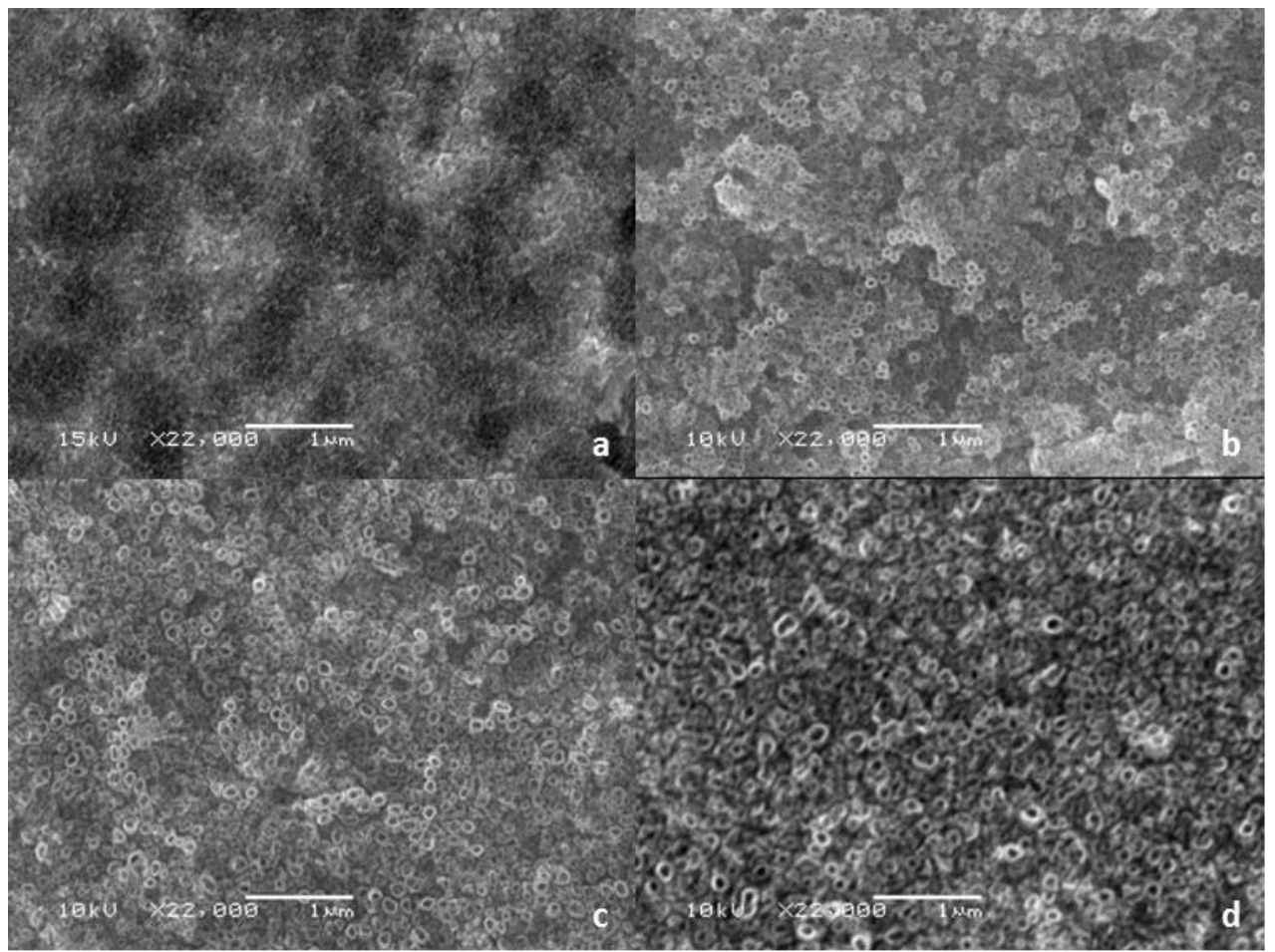

Figure 2. $2 \mathrm{SEM}$ top view images of $\mathrm{TiO}_{2}$ nanotube arrays prepared under different anodization potentials: a) anodization potential $=10 \mathrm{~V}$; b) anodization potential $=15 \mathrm{~V} ; \mathrm{c}$ ) anodization potential $=20 \mathrm{~V} ; \mathrm{d}$ ) anodization potential $=25 \mathrm{~V}$. All samples were annealed at $450{ }^{\circ} \mathrm{C}$ for $30 \mathrm{~min}$.

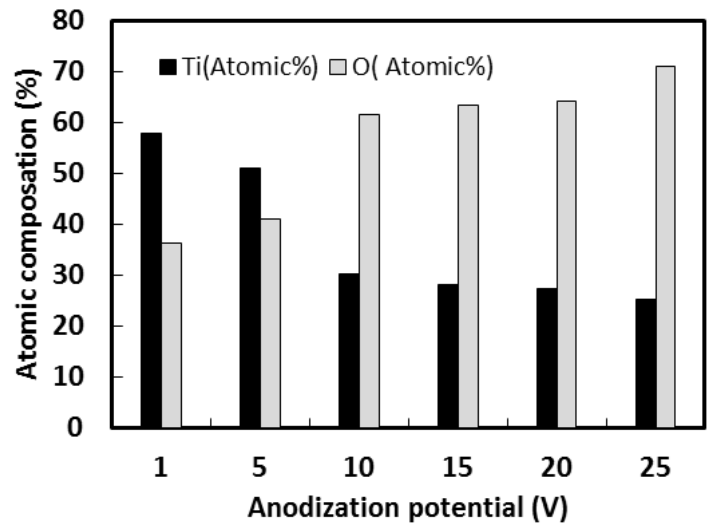

Figure 2. 3 Effect of anodization potential on the atomic composition of anodized Ti foil surface 


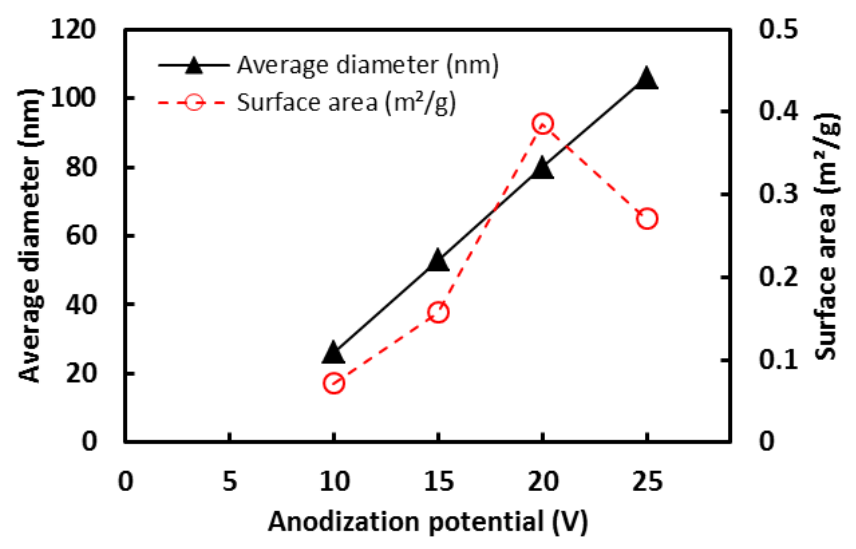

Figure 2. 4 Effect of anodization potential on average $\mathrm{TiO}_{2}$ nanotube diameter and the specific surface area of anodized Ti foil

\subsubsection{Effect of annealing temperature}

Many researchers have reported that as-prepared TNAs are typically mainly in an amorphous form [125, 160, 193], which has low photoactivity. Therefore, annealing is a crucial step to convert the amorphous $\mathrm{TiO}_{2}$ into desired crystalline phase to improve photoactivity of the TNAs. To examine this aspect, TNAs were annealed under three different temperatures. The crystallographic structures were characterized using Raman spectroscopy, as described in the previous section regarding measurement methods. 


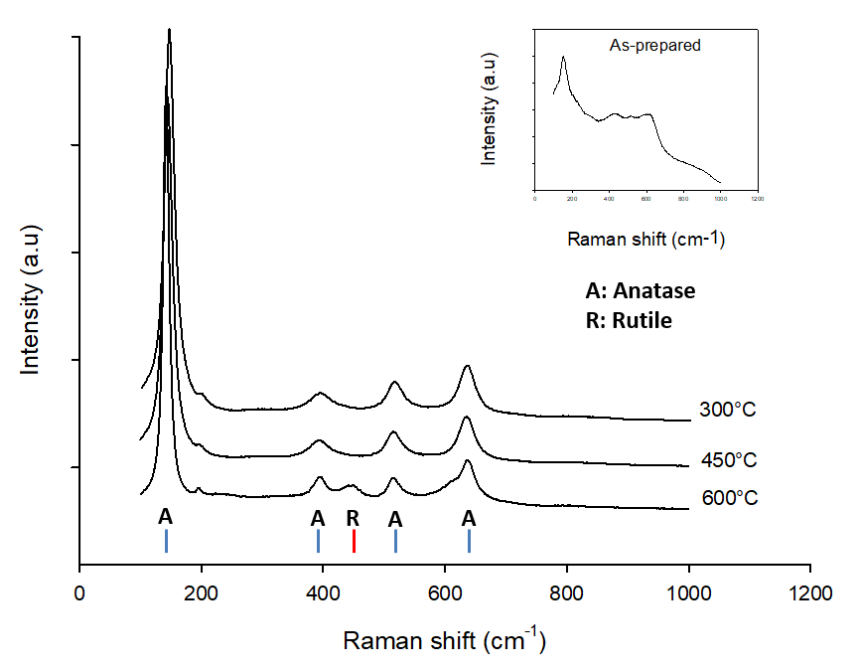

Figure 2. 5 Raman patterns of $\mathrm{TiO}_{2}$ nanotube arrays annealed at different temperatures for $30 \mathrm{~min}$. Raman pattern of as-prepared $\mathrm{TiO}_{2}$ nanotube arrays without annealing is also given, as an inset. Anodization potential $=20 \mathrm{~V}$.

The results (Figure 2. 5) demonstrate that TNAs annealed at $300^{\circ} \mathrm{C}$ and $450^{\circ} \mathrm{C}$ are present in anatase form, while TNAs annealed at $600^{\circ} \mathrm{C}$ have converted to a mixture of rutile and anatase. This is in accordance with previous studies [160]. Interestingly, a peak at around $197 \mathrm{~cm}^{-1}$ was also observed in the as-prepared TNAs, implying the presence of anatase in the as-prepared sample.

\subsubsection{Photocatalytic degradation of metoprolol using $\mathrm{TiO}_{2}$ nanotube arrays as photocatalyst}

\subsubsection{Effect of $\mathrm{TiO}_{2}$ nanotube arrays characterizations}

Although the diameter and crystallographic structure of a TNAs are reported to impose impact on their photocatalytic performance $[162,196]$, the interactions between catalyst and specific target pollutants can be influenced by the structure of the target pollutants [148], and therefore the effect of TNAs properties may differ among different target pollutants. Therefore, the effect of TNAs characterizations on UV-LED/TNAs MTP degradation was investigated experimentally. 


\section{Chapter 2}

\subsubsection{1 $\mathrm{TiO}_{2}$ nanotubes surface morphology}

The effect of $\mathrm{TiO}_{2}$ nanotube diameters on the efficacy of photocatalytic degradation of MTP was evaluated with 4 TNAs produced under anodization potentials ranging from 10 to $25 \mathrm{~V}$, which corresponds to 4 TNAs arrays with average diameters ranging from 26 to $106 \mathrm{~nm}$.

Table 2. 3 Effect of $\mathrm{TiO}_{2}$ nanotube diameters on the kinetics of photocatalytic degradation of metoprolol

\begin{tabular}{cccc}
\hline Nanotube diameter $(\mathbf{n m})$ & $\mathbf{K}_{\text {app }}\left(\mathbf{m i n}^{-1}\right)$ & $\mathbf{R}^{\mathbf{2}}$ & Total removal \\
\hline $\mathbf{2 6}$ & 0.0086 & 0.9927 & $63.66 \%$ \\
$\mathbf{5 3}$ & 0.0144 & 0.9968 & $82.88 \%$ \\
$\mathbf{8 0}$ & 0.0129 & 0.9954 & $79.19 \%$ \\
$\mathbf{1 0 6}$ & 0.0126 & 0.9987 & $78.02 \%$ \\
\hline
\end{tabular}

The results (Figure 2. 6) show that photocatalytic degradations of MTP follow pseudo-first order kinetic model with good correlation $\left(R^{2}>0.99\right)$. The kinetic data and the total removal of MTP after 120 min photocatalytic degradation under different conditions are summarized and shown in Table 2. 3, it should be noticed that all pseudo-first order rate constant values ( $\mathrm{kapp}$ ) in this study were calculated from experimental data of the whole treatment time. From the results shown herein, the $26 \mathrm{~nm}$ diameter TNAs have lowest photoactivity: the pseudo-first order rate constant is $0.0086 \mathrm{~min}^{-1}$, with a total removal of MTP of $63.66 \%$ after $120 \mathrm{~min}$. By increasing the diameter to $53 \mathrm{~nm}$, photoactivity of the TNAs increases significantly: the pseudo-first order rate constant increased by a factor of $67 \%$ to $0.0144 \mathrm{~min}^{-1}$, and the total removal of MTP increased from $63.66 \%$ to $82.88 \%$. 
However, further increasing diameters to $80 \mathrm{~nm}$ and $106 \mathrm{~nm}$ does not exert significant impact on the MTP degradation.

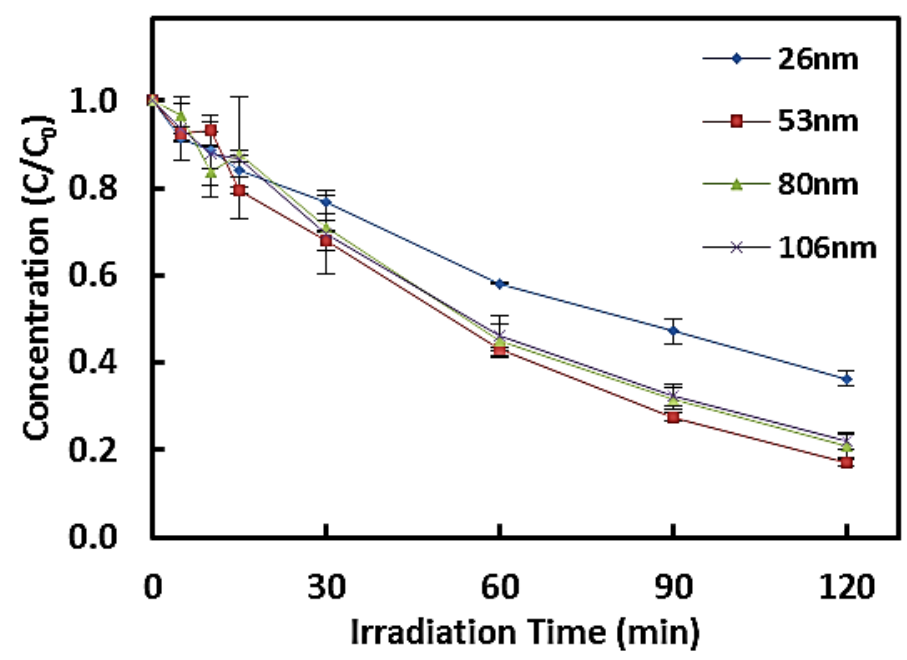

Figure 2. 6 Effect of $\mathrm{TiO}_{2}$ nanotube diameters on the photocatalytic MTP degradation

This finding is in accordance with a previous study conducted by Zhuang et al. [196] using TNAs for photocatalytic degradation of Methyl Orange dye. Those authors have reported that TNAs with diameters of $55 \mathrm{~nm}, 100 \mathrm{~nm}$, and $125 \mathrm{~nm}$ have almost the same photoactivity, which was likely resulted from two facts: (1) the specific surface area of the TNAs decreases with increasing tube diameter resulting in a negative impact; (2) the increasing tube diameter on the other hand increases light penetration and absorption inside the tubular structure which affects the photoactivity positively [196]. In the present study, the MTP degradation increased with increasing tube diameter from $26 \mathrm{~nm}$ to $53 \mathrm{~nm}$, while further increased tube diameters from $53 \mathrm{~nm}$ to $106 \mathrm{~nm}$ impose no significant effect. Increasing nanotube diameter would benefit light transmittance inside the nanotubes, resulting in a positive impact on the photoactivity of TNAs, according to a previous study [196]. Therefore, it is likely that certain nanotube diameter is needed to obtain good photoactivity of TNAs. In addition, the difference in specific surface areas of TNAs with varied diameter may also play a role. The change in specific surface area would not only influence the primary MTP degradation route, which involves reactive species in the liquid phase, by affecting diffusion of reactive species from TNAs surface to the liquid phase, but 


\section{Chapter 2}

also affect the secondary MTP degradation route taking place on the TNAs surface via reaction with $\mathrm{h}^{+}$and surface adsorbed hydroxyl radicals. The phenomenon observed in the present study was a result of the synergetic effect of the abovementioned two facts. To fully understand such synergetic effect, more advanced analysis are required, e.g Electron Paramagnetic Resonance studies to provide more details on the diffusion behaviour of photo-generated reactive species from catalyst surface to the liquid phase.

\subsubsection{2 $\mathrm{TiO}_{2}$ nanotube arrays crystallographic phase}

In addition to tube diameter, crystallographic phase also exerts impact on photoactivity of TNAs [162]. Considering the satisfactory performance of TNAs with diameter $\geq 53 \mathrm{~nm}$, the TNAs prepared under $20 \mathrm{~V}$ which have average diameter of $80 \mathrm{~nm}$ was chosen for further investigations. Performance of TNAs prepared under $20 \mathrm{~V}$ anodization potential with different crystallographic compositions was studied.

Table 2. 4 Effect of $\mathrm{TiO}_{2}$ nanotube arrays crystalline phase compositions on the kinetics of photocatalytic degradation of metoprolol

\begin{tabular}{ccccc}
\hline $\begin{array}{c}\text { Annealing } \\
\text { temperature }\end{array}$ & crystalline phase & $\mathbf{k}_{\text {app }}\left(\mathbf{m i n}^{-1}\right)$ & $\mathbf{R}^{\mathbf{2}}$ & $\begin{array}{c}\text { Total } \\
\text { removal }\end{array}$ \\
\hline $\begin{array}{c}\text { As-prepared } \\
300{ }^{\circ} \mathrm{C}\end{array}$ & Mainly amorphous & 0.0023 & 0.9512 & $25.67 \%$ \\
$450{ }^{\circ} \mathrm{C}$ & Anatase & 0.0118 & 0.9989 & $76.26 \%$ \\
$600{ }^{\circ} \mathrm{C}$ & Anatase & 0.0129 & 0.9954 & $79.19 \%$ \\
\hline
\end{tabular}




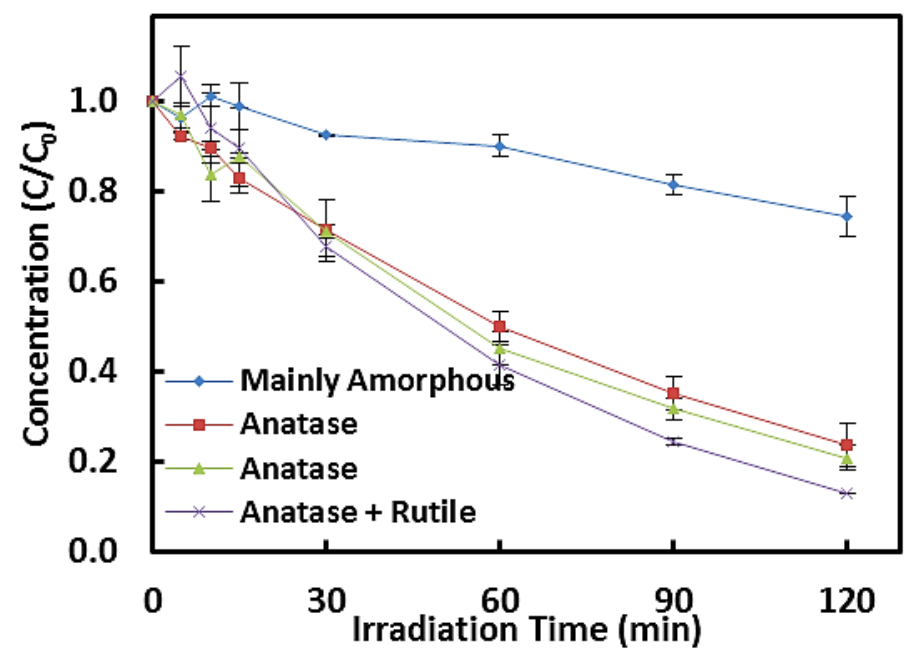

Figure 2. 7 Effect of $\mathrm{TiO}_{2}$ nanotube arrays crystalline phase composition on the photocatalytic MTP degradation

The results (Figure 2. 7 and Table 2. 4) show that crystallographic phase imposes significant impact on the photocatalytic degradation of MTP. Only a small portion (25.67\%) of MTP was degraded over the as-prepared TNAs. This can be attributed to the fact that the as-prepared TNAs are majorly in amorphous phase (although very small amount of anatase is present), which has very low photoresponse. This is in line with literature reporting that amorphous structured $\mathrm{TiO}_{2}$ has very poor photocatalytic efficacy [163]. As aforementioned, by annealing the TNAs at $300{ }^{\circ} \mathrm{C}$ and $450{ }^{\circ} \mathrm{C}$, the $\mathrm{TiO}_{2} \mathrm{NTs}$ were converted to anatase form (Figure 2. 5). Therefore, identical kinetics values and total removal of MTP were obtained, when the TNAs annealed at $300{ }^{\circ} \mathrm{C}$ and $450^{\circ} \mathrm{C}$ were applied. The highest pseudo-first order rate constant and total removal of MTP degradation were obtained when a mixture of anatase and rutile were present. The effect of crystalline phases on the photoactivity of $\mathrm{TiO}_{2}$ remains unclear, and debate still exists because the results of previous studies are not in line with each other. A study conducted by Macak et al. [163]compared the photocatalytic performance of amorphous, anatase phase, rutile phase, mixture of anatase and rutile phases, and the results show that the anatase phase has the highest photocatalytic performance. On the contrary, Liang and Li [203] reported that a mixture of anatase and 


\section{Chapter 2}

rutile has highest photocatalytic performance on degradation of 2,3-dichlorophenol in aqueous solution. Anatase is well known for its better photoactivity. Rutile is known to have smaller band gap than anatase (3.2 eV for anatase and 3.0 eV for rutile) [104], and therefore better response to photons with longer wavelengths can be expected, which could promise an increase in photoactivity of the TNAs with its addition. On the other hand, usually faster recombination of $\mathrm{e}^{-} / \mathrm{h}^{+}$pairs happens in rutile [204] could exerts negative impact on its photoactivity. Therefore, there should be an optimal rutile: anatase ratio, below or above which lower photocatalytic performance is obtained. In depth investigation on this aspect is of interest for optimization of TNAs. Taking into account that the highest pseudo-first order rate constant and total removal of MTP degradation were obtained when a mixture of anatase and rutile were present in TNAs, the TNAs prepared under $20 \mathrm{~V}$ anodization potential and annealed at $600{ }^{\circ} \mathrm{C}$ were used in the following sections.

\subsubsection{Effect of initial concentration of metoprolol}

MTP is reported to be present in various water bodies at very low concentration, at $\mathrm{ng} / \mathrm{L}$ to $\mu \mathrm{g} / \mathrm{L}$ level [103]. Considering this fact, to evaluate the applicability of the photocatalytic degradation of MTP using TNAs, it is of importance to examine how would the initial concentration of MTP affect the efficacy of the UV-LED/TNAs system. Photocatalytic experiments were performed with varied initial MTP concentrations, ranging from $0.1 \mathrm{mg} / \mathrm{L}$ to $1.5 \mathrm{mg} / \mathrm{L}$, to examine this aspect. The efficacy was evaluated in terms of both pseudo-first order kinetic values and total removal of MTP after 120 min treatment.

Table 2. 5 Effect of initial concentration of metoprolol on the kinetics of photocatalytic metoprolol degradation

\begin{tabular}{cccc}
\hline Initial MTP concentration (mg/L) & $\mathbf{k}_{\text {app }}\left(\mathbf{m i n}^{-1}\right)$ & $\mathbf{R}^{\mathbf{2}}$ & Total removal \\
\hline 0.1 & 0.0185 & 0.9984 & $89.27 \%$ \\
0.5 & 0.0165 & 0.9968 & $87.03 \%$ \\
1.0 & 0.0161 & 0.9819 & $87.09 \%$ \\
1.5 & 0.0108 & 0.9780 & $74.50 \%$ \\
\hline
\end{tabular}




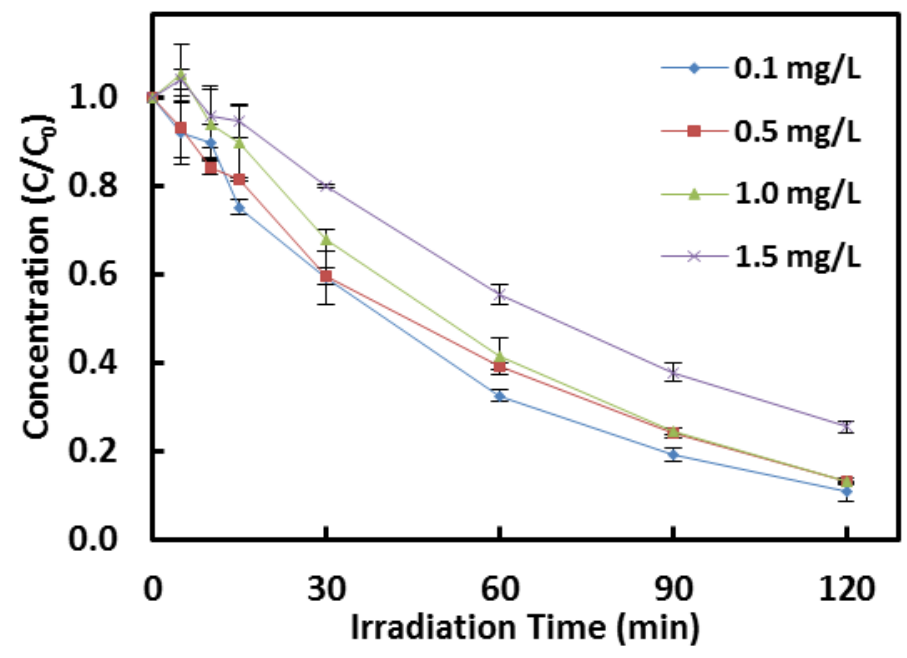

Figure 2. 8 Effect of initial concentration of metoprolol on the photocatalytic MTP degradation

Unsurprisingly, the results (Figure 2. 8 and Table 2. 5) show that both the degradation rate constant and total removal of MTP were not negatively affected by decreasing initial MTP concentration from $1.0 \mathrm{mg} / \mathrm{L}$, and the MTP degradations followed pseudo-first order kinetics model when initial MTP concentration was in the range of $0.1-1.0 \mathrm{mg} / \mathrm{L}$. On the other hand, decreased apparent rate constant and total removal of MTP were observed when initial MTP concentration was increased to $1.5 \mathrm{mg} / \mathrm{L}$. This finding can be attributed to the following facts: (1) the availability of reactive species was constant under the same applied irradiation conditions and catalyst loading [99]; (2) when initial MTP concentration was $1.0 \mathrm{mg} / \mathrm{L}$, the amount of reactive species was already at exceed level, resulting in pseudo-first order degradation of MTP. When lower initial MTP concentration was applied, the reactive species to target pollutant ratio was further increased, therefore at lower initial MTP concentration the MTP degradation still followed the same pseudo-first order kinetics model. Consequently, lower initial MTP concentration imposed no negative impact on MTP degradation. The analysis presented here is an indication that application of the UV-LED/TNAs photocatalytic degradation system in real-life may not be negatively affected by much more diluted MTP concentration, and it could assist further 


\section{Chapter 2}

comprehensive studies assessing the performance of the UV-LED/TNAs system in real life implications.

\subsubsection{Effect of $\mathrm{pH}$}

The effect of $\mathrm{pH}$ was examined in the range of 3 to 11 to study the applicability of the TNAs based photocatalytic pharmaceutical degradation processes, in terms of both degradation kinetics and total removal after 120 min treatment.

Table 2. 6 Effect of initial pH on the kinetics of photocatalytic metoprolol degradation

\begin{tabular}{cccc}
\hline Initial $\mathbf{p H}$ & $\mathbf{K}_{\text {app }}\left(\mathbf{m i n}^{-1}\right)$ & $\mathbf{R}^{\mathbf{2}}$ & Total removal \\
\hline 3 & 0.0151 & 0.9975 & $84.29 \%$ \\
6 & 0.0161 & 0.9819 & $87.09 \%$ \\
11 & 0.0104 & 0.9824 & $73.83 \%$ \\
\hline
\end{tabular}

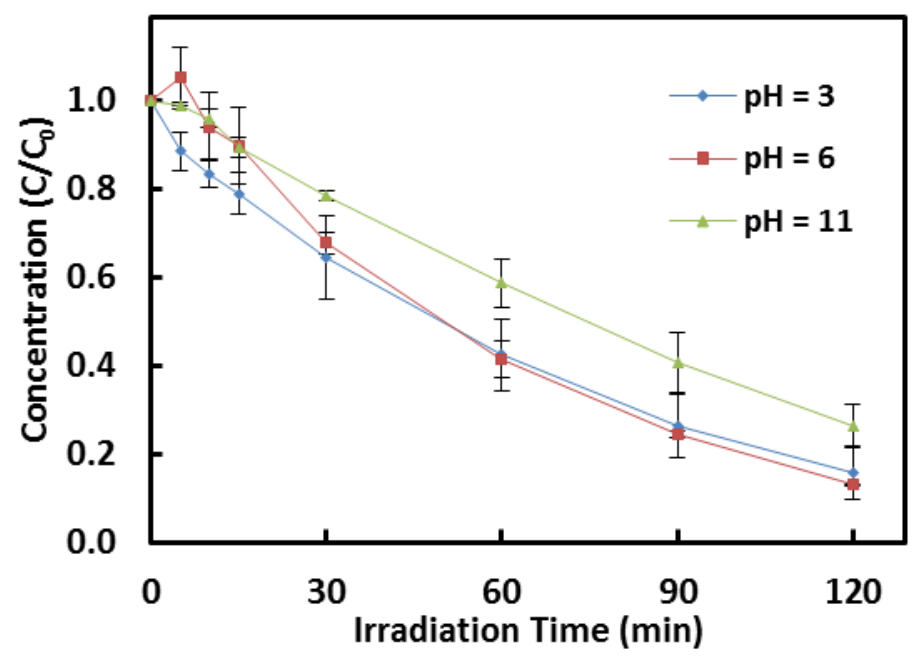

Figure 2. 9 Effect of initial pH on the photocatalytic MTP degradation

The results (Table 2. 6 and Figure 2. 9) reveal that the photocatalytic performance of TNAs on MTP degradation remains highly effective in a wide $\mathrm{pH}$ range, from 3 to 11 . As demonstrated in Table 2. 6 and Figure 2. 9, the UV-LED/TNAs system can be applied in a 
wide $\mathrm{pH}$ range: (1) even acute acidic conditions $\left(\mathrm{pH}_{0}=3\right)$ imposes no adverse impact on the photocatalytic degradation of MTP; (2) a moderate decrease in MTP degradation was observed under acute basic conditions $\left(\mathrm{pH}_{0}=11\right)$, but the overall performance was still comparable to that under neutral and acidic conditions. Plausible explanation of the decrease of photocatalytic MTP degradation over TNAs lies in the electrostatic repulsive effect between deprotonated MTP and TNAs surface. MTP has a $\mathrm{pK}_{\mathrm{a}}$ value of 9.68 [205], making it deprotonated into negatively charged anion form. Meanwhile, under acute basic conditions $\left(\mathrm{pH}_{0}=11\right)$ the TNAs surface was also negatively charged, according to literature [166]. Consequently, the electrostatic effect would make TNAs surface repulsive to the deprotonated MTP, which would pose a negative effect on the degradation of MTP. As addressed in the mechanistic section, although major MTP degradation occurred in the bulk liquid phase, secondary MTP degradation routes occurred on the surface TNAs (TNAs - liquid interface), involving either direct oxidation by photo generated holes or hydroxyl radicals adsorbed on TNAs surface. The high $\mathrm{pH}$ induced repulsive effect of the TNAs surface towards deprotonated MTP molecules resulted in inhibition of the secondary MTP degradation route. The same effect of alkaline $\mathrm{pH}$ has been reported in a study conducted by Habibi et al. [106], where the effect of $\mathrm{pH}$ on slurry $\mathrm{TiO}_{2}$ photocatalytic degradation of Reactive Yellow 2 was examined and increasing $\mathrm{pH}$ was reported to cause decreased degradation efficiency of the dye.

\subsubsection{Effect of inorganic ions}

Regarding real life application of heterogeneous photocatalytic systems, presence of various inorganic ions should be taken into account, because many of them have been reported to pose detrimental effect even at low concentration levels [96, 106, 166]. Therefore, experiments were carried out to examine the effect of presence of some common inorganic ions, i.e. bicarbonate ions, phosphate ions. To best represent the real implication, $\mathrm{HCl}$ was used to adjust the reaction solutions with phosphate or bicarbonate addition to circum-neutral (6-7). 


\section{Chapter 2}

Table 2. 7 Effect of inorganic ions on the kinetics of photocatalytic metoprolol degradation

\begin{tabular}{|c|c|c|c|c|}
\hline $\begin{array}{c}\text { Background water } \\
\text { constituents }\end{array}$ & $\begin{array}{l}\text { Concentration } \\
\text { (mg/L) }\end{array}$ & $K_{a p p}\left(\min ^{-1}\right)$ & $\mathbf{R}^{2}$ & $\begin{array}{c}\text { Total } \\
\text { removal }\end{array}$ \\
\hline \multirow[t]{2}{*}{ No addition } & 0 & 0.0161 & 0.9819 & $87.09 \%$ \\
\hline & 50 & 0.0194 & 0.9956 & $90.78 \%$ \\
\hline \multirow[t]{3}{*}{ Bicarbonate } & 100 & 0.0154 & 0.9941 & $85.19 \%$ \\
\hline & 200 & 0.0149 & 0.9913 & $84.57 \%$ \\
\hline & 50 & 0.0219 & 0.9970 & $93.26 \%$ \\
\hline \multirow[t]{2}{*}{ Phosphate } & 100 & 0.0231 & 0.9947 & $94.28 \%$ \\
\hline & 200 & 0.0206 & 0.9965 & $92.19 \%$ \\
\hline
\end{tabular}

The obtained results (Figure 2. 10 and Table 2. 7) show that the presence of both inorganic ions exerts no detrimental impact on the UV/TNAs photocatalytic degradation of MTP. Both the pseudo-first order rate constant and the total removal of MTP degradation were not negatively influenced by the presence of phosphate ions or bicarbonate ions at various concentration levels. Moreover, slight increase in the MTP degradation was obtained with the presence of phosphate ions or $50 \mathrm{mg} / \mathrm{L}$ bicarbonate ions. The obtained results herein exhibit great advantage of $\mathrm{TNAs}$ over conventional $\mathrm{TiO}_{2}$ slurry systems. Significant detrimental effect of bicarbonate and phosphate ions on the performance of conventional $\mathrm{TiO}_{2}$ slurry systems has been well documented in literature and examples are abundant [106, 166-168]. In a study conducted by Rioja et al. [167] investigated the effect of water matrix on the photodegradation of clofibric acid, and found that even the presence of $50 \mathrm{mg} / \mathrm{L} \mathrm{NaHCO}_{3}$ or $\mathrm{NaCO}_{3}$ caused significant decrease in the clofibric acid degradation. Another study conducted by Rincon et al. [168] investigated the effect of inorganic on photocatalytic inactivation of E. Coli, and reported that addition of bicarbonate and phosphate ions resulted in a meaningful decrease in photocatalytic $E$. Coli inactivation. 

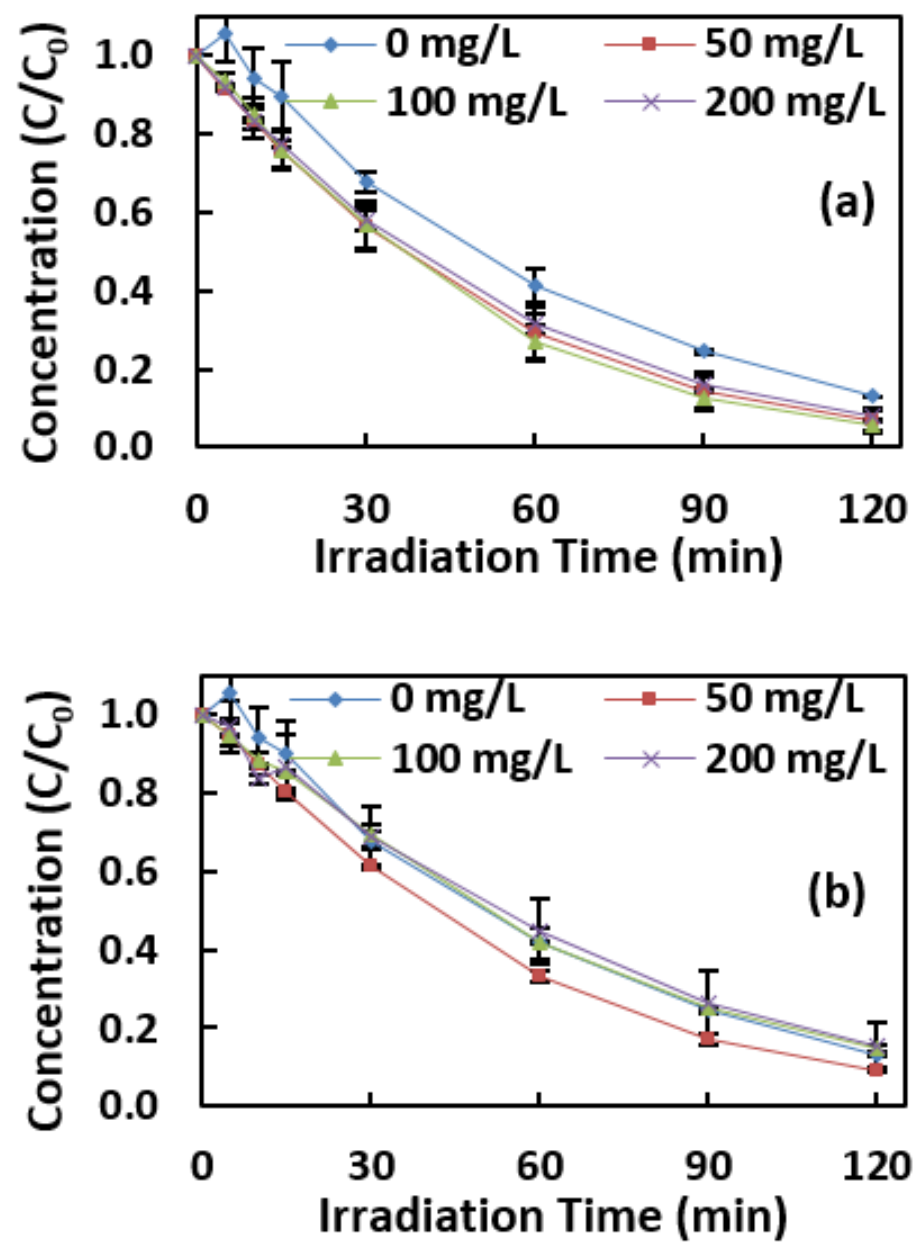

Figure 2. 10 Effect of inorganic ions on the photocatalytic MTP degradation: (a) phosphate ions (b) bicarbonate ions

Although there is a generally known detrimental effect of carbonate species $\left(\mathrm{HCO}_{3}{ }^{-}\right.$or $\left.\mathrm{CO}_{3}{ }^{2-}\right)$ and phosphate species on AOPs, mainly due to scavenging of hydroxyl radicals, the possible positive impact should also be taken into account. For the carbonate species, by reacting with hydroxyl radicals, carbonate radicals are generated $[206,207]$. The carbonate radical has relatively high oxidizing potential $\left(E_{0}=1.78 \mathrm{~V}\right.$ at $\mathrm{pH}$ ) and tend to attack compounds with electron-rich moieties [206]. Besides, carbonate species can also act as conduction band electrons quencher [208], which decreases the recombination of 


\section{Chapter 2}

the $\mathrm{e}^{-} / \mathrm{h}^{+}$pairs and can in turn impose a positive impact on the photocatalytic degradation of MTP. To confirm the electron quenching capability of bicarbonate species, an additional experiment was conducted by irradiating a $200 \mathrm{mg} / \mathrm{L}$ bicarbonate aqueous solution with UV light in the presence of TNA. The obtained results (Table S2. 1) show that after $120 \mathrm{~min}$ irradiation, the total organic carbon of the solution increased from $0 \mathrm{mg} / \mathrm{L}$ to nearly $5 \mathrm{mg} / \mathrm{L}$, which soundly support the hypothesis that the bicarbonate ions in the UV/TNAs system are able to rigorously quench conduction band electrons. To this end, the detrimental effect of bicarbonate induced by scavenging of hydroxyl radicals would be compensated by the formation of carbonate radicals and following carbonate radical mediated MTP degradation, as well as the quenching of conduction band electrons by bicarbonate ions. Therefore, the presence of bicarbonate in this study had no detrimental effect. For the presence of phosphate, the effect is also two-sided. On the one hand, it can adsorb on to the catalyst surface to decrease the adsorption of target pollutants and therefore hinder the degradation of target pollutants on the catalyst surface. However, previous studies have also pointed out that the adsorption of phosphate ions on the catalyst surface would lead to enhanced photocatalytic degradation of target pollutants by promoting the separation of $\mathrm{e}^{-/ \mathrm{h}^{+}}$pairs via introducing electrostatic field on the catalyst surface [209]. Considering the fact that major MTP degradation takes place in the bulk liquid phase (as described in details in section 2.3.4), the adverse effect of phosphate can be neglected. Therefore, a slight increase in MTP degradation was obtained with presence of phosphate ions.

\subsubsection{Effect of NOMs}

Natural organic matters (NOMs) are present in broad range of water bodies and are known to be able to interfere with AOPs [177]. Given this context, to evaluate the applicability of TNAs for photocatalytic degradation of MTP, it is of interest to study the effect of NOMs and document the bearable NOMs concentrations range. Experiments were carried out in the presence of various NOMs concentrations from $5 \mathrm{mg} / \mathrm{L}$ to $15 \mathrm{mg} / \mathrm{L}$ (corresponding to TOC concentrations from $2 \mathrm{mg} / \mathrm{L}$ to $6 \mathrm{mg} / \mathrm{L}$ ). 
Table 2. 8 Effect of NOMs on the kinetics of photocatalytic metoprolol degradation

\begin{tabular}{cccc}
\hline NOMs concentration $(\mathbf{m g} / \mathbf{L})$ & $\mathbf{K}_{\text {app }}\left(\mathbf{m i n}^{-1}\right)$ & $\mathbf{R}^{\mathbf{2}}$ & Total removal \\
\hline 0 & 0.0161 & 0.9819 & $87.09 \%$ \\
5 & 0.0147 & 0.9967 & $83.72 \%$ \\
10 & 0.0053 & 0.9730 & $49.42 \%$ \\
15 & 0.0040 & 0.9889 & $38.93 \%$ \\
\hline
\end{tabular}

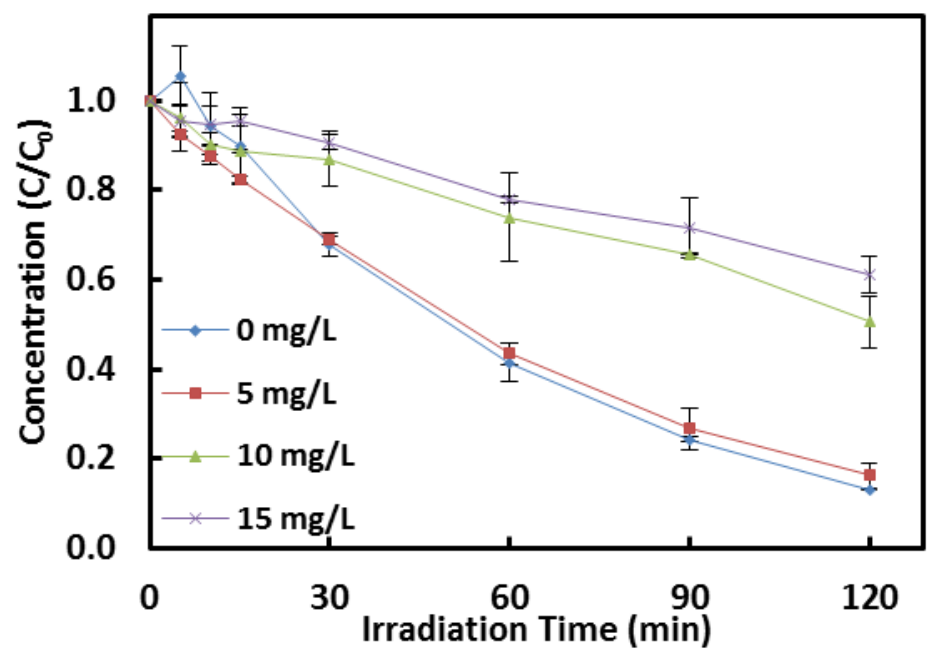

Figure 2. 11 Effect of NOMs on the photocatalytic MTP degradation

The obtained results (Figure 2. 11 and Table 2. 8) indicate that the presence of NOMs at concentration levels $\leq 5 \mathrm{mg} / \mathrm{L}$ has no noteworthy detrimental effect. Both the pseudo-first-order rate constant and total removal of MTP remained at the same level, when $5 \mathrm{mg} / \mathrm{L}$ NOMs was added. With increased concentration levels, the NOMs started to exhibit a detrimental effect on the photocatalytic MTP degradation. The degradation rate constant decreased drastically from $0.0147 \mathrm{~min}^{-1}$ to $0.0053 \mathrm{~min}^{-1}$ when NOMs concentration increased from $5 \mathrm{mg} / \mathrm{L}$ to $10 \mathrm{mg} / \mathrm{L}$, and the total removal of MTP decreased from $83.72 \%$ to 49.42\%. Further decrease in MTP degradation was observed by increasing NOMs concentration to $15 \mathrm{mg} / \mathrm{L}$ : the degradation rate constant decreased to $0.0040 \mathrm{~min}^{-1}$ and the total removal of MTP decreased to $38.93 \%$. 


\section{Chapter 2}

This observed phenomenon can be attributed to the characteristics of NOMs. The NOMs have light absorption in UV and near UV range (See Figure S2. 3 in supplementary materials). Moreover, NOMs can also act as reactive species quencher, which in turn decrease the availability of reactive species for MTP degradation. Therefore, they impose a detrimental effect on photocatalytic MTP degradation over TNAs.

Although it is also documented that NOMs can act as photosensitizer which may mitigate its detrimental effect on target pollutant degradation, the experimental data (Figure S2. 4) suggest that NOMs do not act as photosensitizer in the UV-LED/TNAs system under the applied conditions.

\subsubsection{Efficacy of the $\mathrm{TiO}_{2}$ nanotube arrays on photocatalytic metoprolol degradation in} tap water

To further evaluate the applicability of the TNAs, an experiment was carried out with tap water to examine the efficacy of photocatalytic MTP degradation by using TNAs as photocatalyst. The pseudo-first order kinetic constant and total removal of MTP degradation demonstrated in Figure 2. 12 and Table 2. 9. The results show that the degradation rate constants experienced a decrease from $0.0161 \mathrm{~min}^{-1}$ in Milli-Q water to the value of $0.0076 \mathrm{~min}^{-1}$ in tap water. The total removal of MTP after $120 \mathrm{~min}$ of photocatalytic treatment over TNAs decreased from $87.09 \%$ in Milli-Q water to $62.05 \%$ in tap water.

Table 2. 9 Comparison of the kinetics of photocatalytic metoprolol degradation in tap water and Milli-Q water

\begin{tabular}{cccc}
\hline Water matrix & $\mathbf{K a p p}\left(\mathbf{m i n}^{-1}\right)$ & $\mathbf{R}^{\mathbf{2}}$ & Total removal \\
\hline Milli-Q & 0.0161 & 0.9819 & $87.09 \%$ \\
Tap water & 0.0076 & 0.9849 & $62.05 \%$ \\
\hline
\end{tabular}




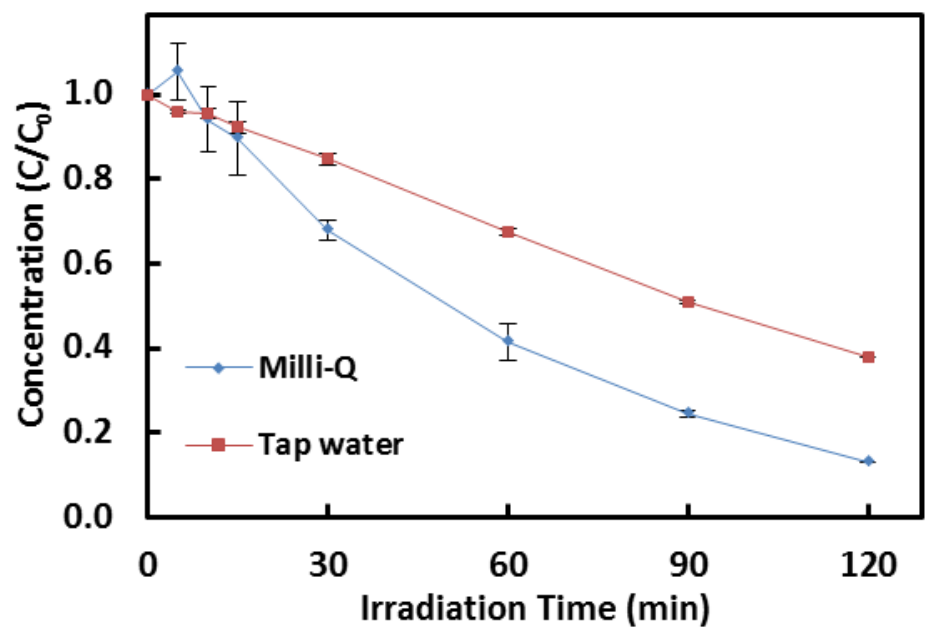

Figure 2. 12 Effect of water matrices on the photocatalytic MTP degradation

The characteristics of the tap water were shown in Table 2. 2. Although bicarbonate and phosphate ions exhibit no detrimental effect on the photocatalytic degradation of MTP over TNAs as shown in previous section, the degradation of MTP would still be interfered by the presence of other organic compounds in tap water. Those organic compounds would compete with the target pollutant for oxidizing species, screen UV irradiation, and may also adsorb onto the photocatalyst surface [119]. On the contrary to TNAs based, conventional $\mathrm{TiO}_{2}$ slurry system was much more vulnerable to the mater matrix. In a previous study conducted by Rioja et al. [167], it is reported that the target pollutant degradation rate constant decreased drastically from $0.3468 \mathrm{~min}^{-1}$ in pure water to 0.0033 $\mathrm{min}^{-1}$ in tap water.

\subsubsection{Contribution of different photo-induced reactive species}

Previous studies on $\mathrm{TiO}_{2}$ based photocatalytic systems have pointed out that hydroxyl radicals are vital reactive species in degradation of target-pollutants [108]. However, some researchers clearly pointed out that other species, including electrons, holes, superoxide anion radicals, singlet oxygen, can also contribute to degradation of target-pollutants depending on the characteristics of specific catalyst [104]. Viewing this 


\section{Chapter 2}

fact, in order to evaluate the mechanistic aspect, experiments with addition of specific scavengers were carried out. The results demonstrate that the degradation of MTP was greatly hindered: total removal after 120 min treatment was decreased to $2.42 \%$ and $10.48 \%$, with addition formic acid and tert-butanol respectively.

Knowing the fact that formic acid can rigorously quench photo generated electron holes $\left(\mathrm{h}^{+}\right)$on the surface of TNAs once they were produced, addition of formic acid in the reaction hindered not only the degradation pathway mediated by electron holes but also degradation pathways with participation of hydroxyl radicals [104]. Due to extreme low affinity to $\mathrm{TiO}_{2}$ surfaces, the tert-butanol only scavenges hydroxyl radicals in bulk liquid phase. From the obtained results, the contribution of different degradation routes was obtained. As shown in Figure 2. 13, the primary photocatalytic degradation of MTP occurred via reaction with hydroxyl radicals in bulk liquid phase, which accounts for $88 \%$ of the MTP removal. In a recent study conducted by Arlos et al.[148], it is reported that highly mobility of hydroxyl radicals allows them diffuse from the surface to liquid phase and therefore interact with compounds in the liquid phase, and hence adsorption of target contaminants on catalyst surface is not necessarily a pre-requisite for efficient photocatalytic degradation of target-pollutants. This is in accordance with the present study: only very small portion of MTP degradation occurred on the surface of TNAs (TNAs - liquid interface), degraded by either direct oxidation by photo generated holes or hydroxyl radicals adsorbed on TNAs surface. Additionally, it should be noticed that other reactive species, likely to be superoxide anion radicals according to literature [104], did also play a minor role in MTP degradation over TNAs, which accounts for around $3 \%$ of the MTP removal. This finding in this study is in agreement with previous studies on $\mathrm{TiO}_{2}$ photocatalytic MTP removal processes [104]. A schematic of the MTP degradation over TNAs was proposed as shown in Figure 2. 14. 


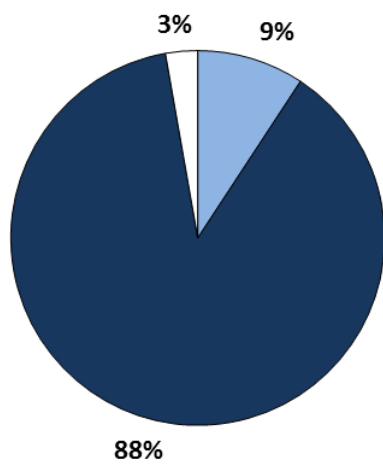

$\square \mathrm{h}+$ and adsorbed $\cdot \mathrm{OH}$ on

TNAs surface

$\square$ free $\cdot \mathrm{OH}$ in liquid phase

$\square$ Other reactive species

Figure 2. 13 Contribution of different reactive species on the MTP degradation

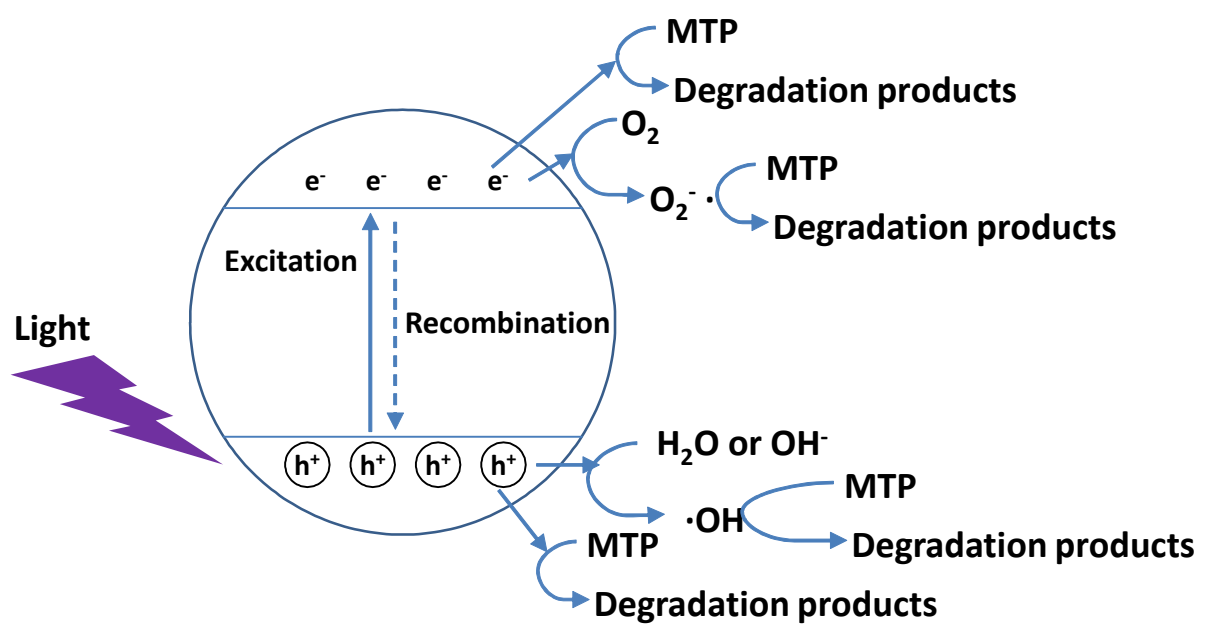

Figure 2. 14 Proposed schematic photocatalytic degradation mechanism of MTP over TNAs

This finding was further confirmed via by-products analysis by LC-MS/MS. The LCMS/MS analysis show that the UV/TNAs photocatalytic degradation of MTP resulted in formation of 25 main degradation by-products (DP). The $\mathrm{m} / \mathrm{z}$ ratios and corresponding retention times (RT) of detected DPs are summarized in Table S2. 2, and the evolution of those DPs versus time was summarized in Figure S2. 7. The obtained DPs are in agreement with previous studies using $\mathrm{TiO}_{2}$ for MTP removal [103, 104, 119], and tentative structures of those DPs have been proposed by a previous study [119]. By inhibiting the participation of holes and $\cdot \mathrm{OH}$, most of the DPs were not formed, and only DP3 (m/z=134, RT=1.22 $\mathrm{min}$ ), 


\section{Chapter 2}

DP4 (m/z=76, RT=1.23 $\mathrm{min})$, DP6 (m/z=120, RT=1.23 $\mathrm{min})$, and DP23 (m/z=284, RT=5.45 $\mathrm{min}$ ) were observed. By inhibiting the participation of $\cdot \mathrm{OH}$ in the bulk liquid phase, DP3, DP4, DP6, DP23, and DP24 (m/z=282, RT=5.66 min) were obtained.

\subsubsection{Stability of $\mathrm{TiO}_{2}$ nanotube arrays}

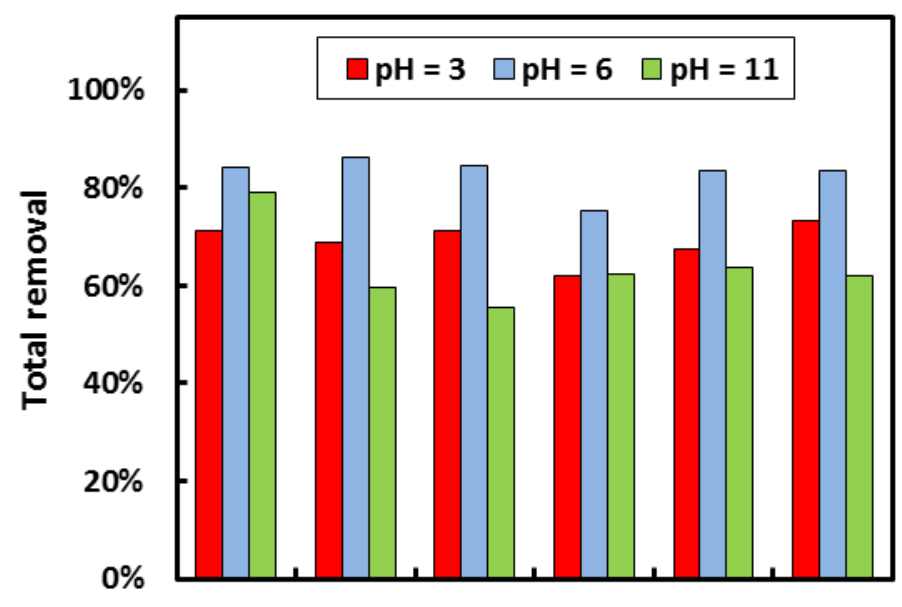

1st run 2 nd run 3 rd run 4 th run 5 th run 6 th run

Figure 2. 15 Total removal of MTP after $120 \mathrm{~min}$ photocatalytic treatment over TNAs for repeated use at different $\mathrm{pH}$ conditions. ([MTP $]_{0}=1 \mathrm{mg} / \mathrm{L}$.)

Stability of the TNAs was evaluated under acidic $\left(\mathrm{pH}_{0}=3\right)$, neutral $\left(\mathrm{pH}_{0}=6\right)$, and alkaline conditions $\left(\mathrm{pH}_{0}=11\right)$ in terms of their reuse performance over 6 cycles. For each cycle, the treatment time was $120 \mathrm{~min}$. The TNAs were washed by Milli-Q water and dried in $\mathrm{N}_{2}$ stream at ambient temperature after each run, and were then reused in subsequent run. As demonstrated in Figure 2. 15, photocatalytic MTP degradation by TNAs was not negatively affected during the repeated treatment cycles under different operation conditions, revealing relatively good stability of the TNAs and its potential in real life implications. 


\subsection{Conclusions}

The synthesis of self-organized $\mathrm{TiO}_{2}$ nanotube arrays (TNAs) and their application for photocatalytic degradation of a $\beta$-blocker metoprolol (MTP) was studied. Self-organized TNAs are found to be a powerful catalyst for photocatalytic removal of MTP from aqueous streams. Main conclusions can be drawn from our results are summarized herein:

* For fabrication of self-organized TNAs, anodization potential and annealing temperature are two key parameters which control the diameter and crystallographic composition of the anodic produced $\mathrm{TiO}_{2}$ nanotubes respectively.

* The surface morphology and crystallographic composition of the anodic produced $\mathrm{TiO}_{2}$ nanotubes impose impact on performance of the TNAs in photocatalytic MTP degradation, respectively. For TNAs with given average nanotube diameters, photocatalytic MTP degradation was favoured when a mixture of anatase and rutile was present in the TNAs structure; for TNAs with certain crystallographic composition, rapid MTP degradation was obtained when the nanotubes diameter reached $53 \mathrm{~nm}$.

* The UV-LED/TNAs system can be applied in a wide $\mathrm{pH}$ range, and lower initial MTP concentration imposed no negative impact on MTP degradation. The analysis presented here is an indication that application of the UV-LED/TNAs photocatalytic degradation system in real-life may not be negatively affected by much more diluted MTP concentration, and it could assist further comprehensive studies assessing the performance of the UV-LED/TNAs system in real life implications.

* Regarding real life application, the presence of even high level bicarbonate ions and phosphate ions has no detrimental effect, while NOMs concentration higher than $5 \mathrm{mg} / \mathrm{L}$ does negatively affect the photocatalytic degradation of MTP. Operation in real tap water samples only led to a slight decrease in the process efficiency.

* The obtained results demonstrate that primary degradation process occurred in liquid phase with participation of hydroxyl radicals in the liquid phase 
$\left(\cdot \mathrm{OH}_{\text {liquid }}\right)$, while smaller portion of MTP were degraded on the catalysis surface via reaction with $\mathrm{h}^{+}$and hydroxyl radicals adsorbed on the catalyst surface $(\cdot \mathrm{OH}$ surface). Other reactive species, e.g photo generated electrons and superoxide radical anions, did also play a minor role in MTP degradation. The mechanistic aspect was further confirmed by identification of degradation products by LC-MS/MS.

* The TNAs exhibit good stability during repeated treatment cycles, which is an indication of its potential in real life implications. To fully understand its applicability in real life implications, further research efforts are required, for instance the performance of TNAs during long time operation in the presence of various inorganics and other pollutants, and also the synergetic effect of coexistence of inorganics and other organics on the performance of the catalyst and cost of operation of the system should be evaluated systematically.

\subsection{Acknowledgements}

This work was performed in the cooperation framework of Wetsus, European Centre of Excellence for Sustainable Water Technology (www.wetsus.eu). Wetsus is cofunded by the Dutch Ministry of Economic Affairs and Ministry of Infrastructure and Environment, the Province of Fryslân, and the Northern Netherlands Provinces. The authors would like to thank the participants of the research theme "Advanced water treatment" for the fruitful discussions and their financial support. The authors gratefully thank Ton van der Zande and Mieke Kersaan-Haan for the realization of the instrumental analysis, and Dr. Sofia Semitsoglou-Tsiapou and Dr. Maarten Biesheuvel for the fruitful discussions. 


\section{Supplementary information}

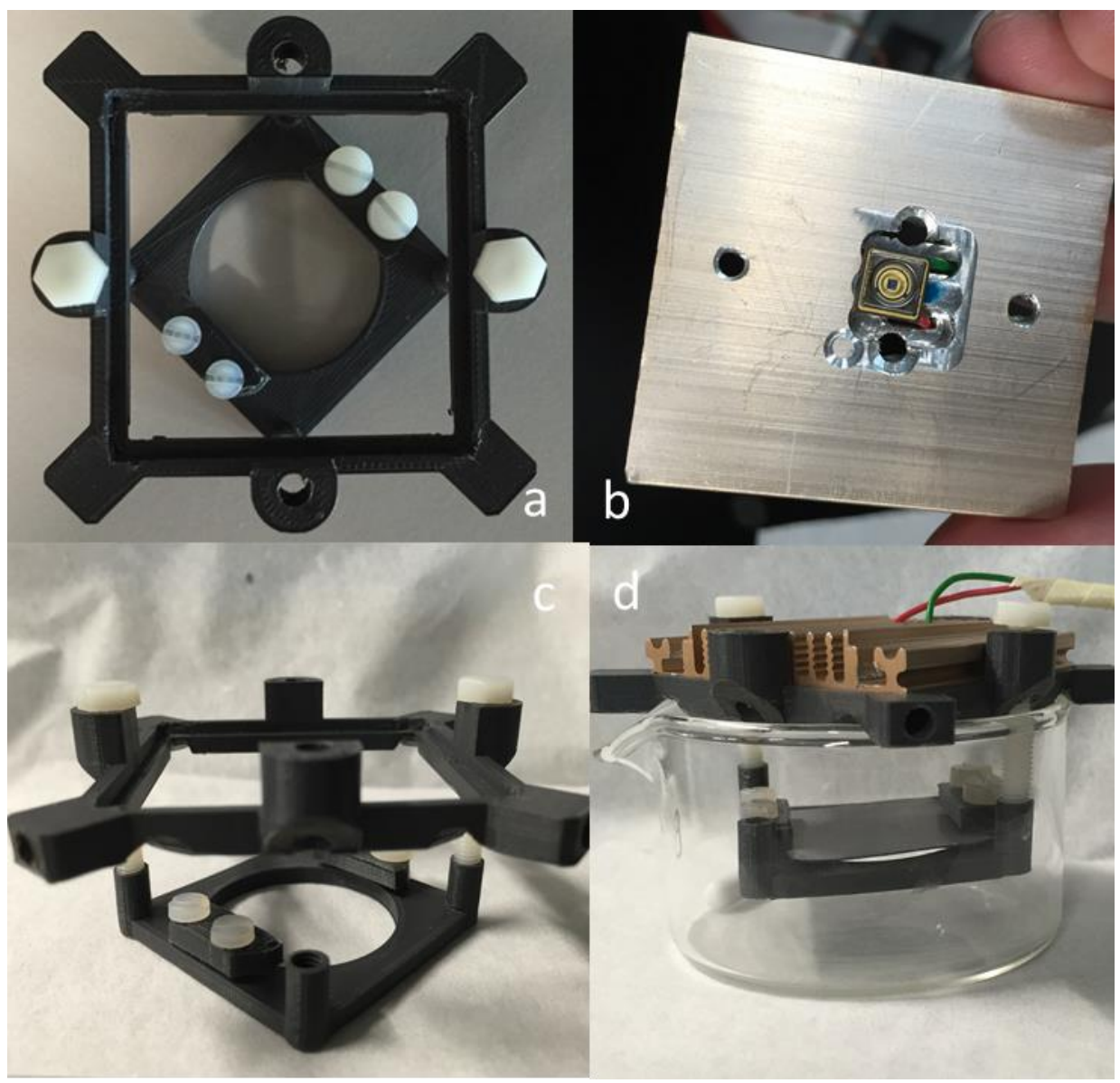

Figure S2. 1 Photo of the set-up: (a) top view of the frame for fixing TNAs and UV-LED;

(b) UV-LED fixed on the aluminum heat dispenser; (c) side view of the frame for fixing TNAs and UV-LED; (d) side view of the set-up 
Chapter 2

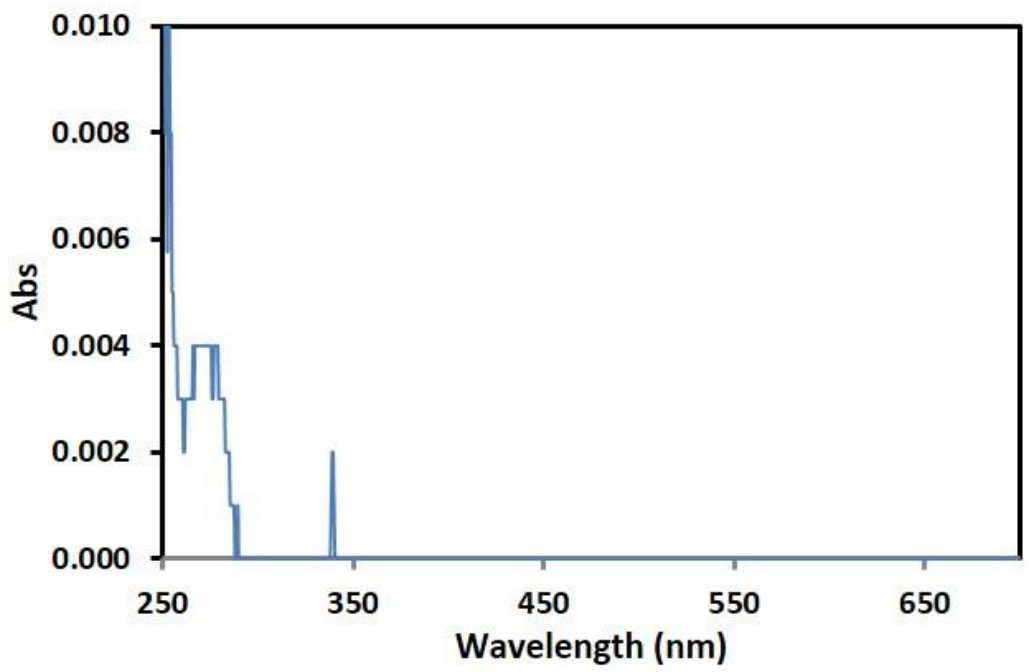

Figure S2. 2 Light absorbance spectrum of MTP

Table S2. 1 Total organic carbon (TOC) level before and after UV/TNAs treatment.

$\left([\mathrm{MTP}]_{0}=0 \mathrm{mg} / \mathrm{L},\left[\mathrm{HCO}_{3}{ }^{-}\right]_{0}=200 \mathrm{mg} / \mathrm{L}\right.$, circum-neutral)

\begin{tabular}{cc}
\hline Irradiation time $(\min )$ & TOC $(\mathrm{mg} / \mathrm{L})$ \\
\hline $\mathbf{0}$ & 0 \\
120 & 4.95 \\
\hline
\end{tabular}


Chapter 2

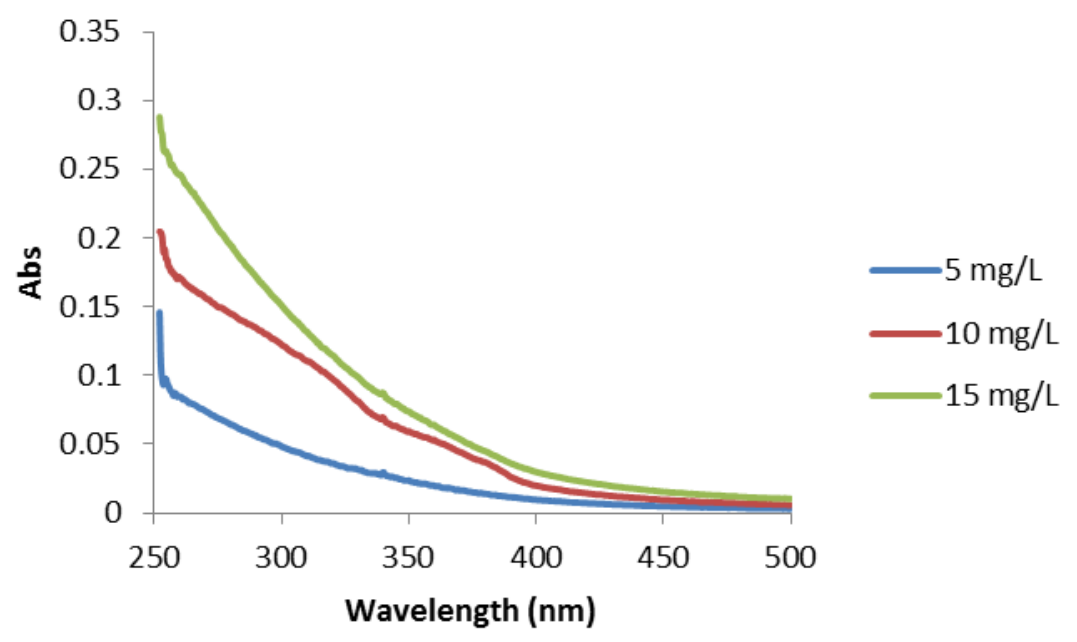

Figure S2. 3 Light absorbance spectrum of NOMs at different concentration levels

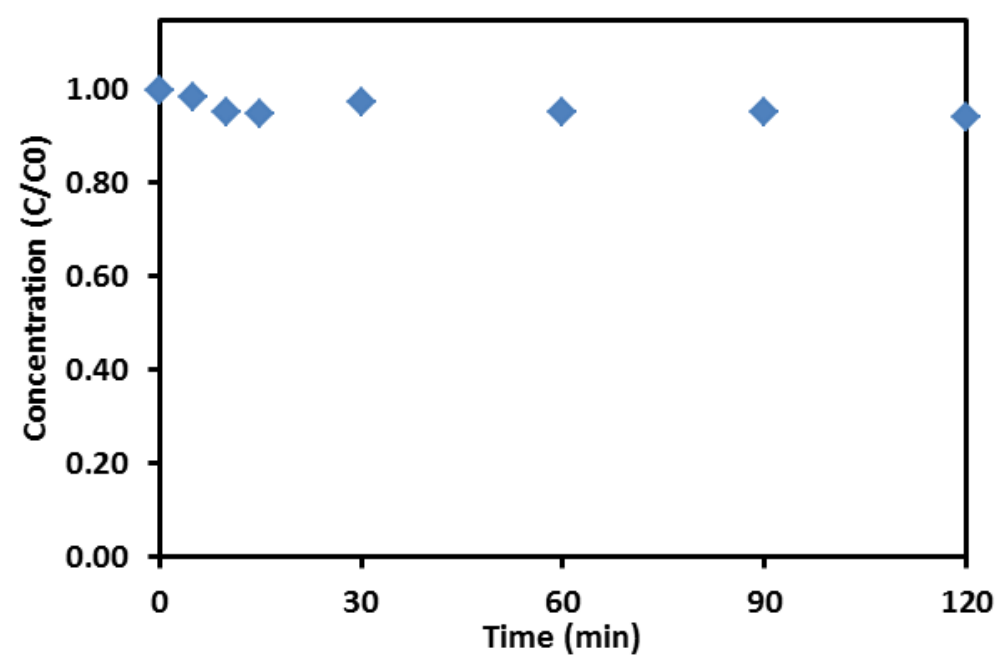

Figure S2. 4 MTP concentration profile as a function of time under UV irradiation with presence of $5 \mathrm{mg} / \mathrm{L}$ NOMs, without TNAs 


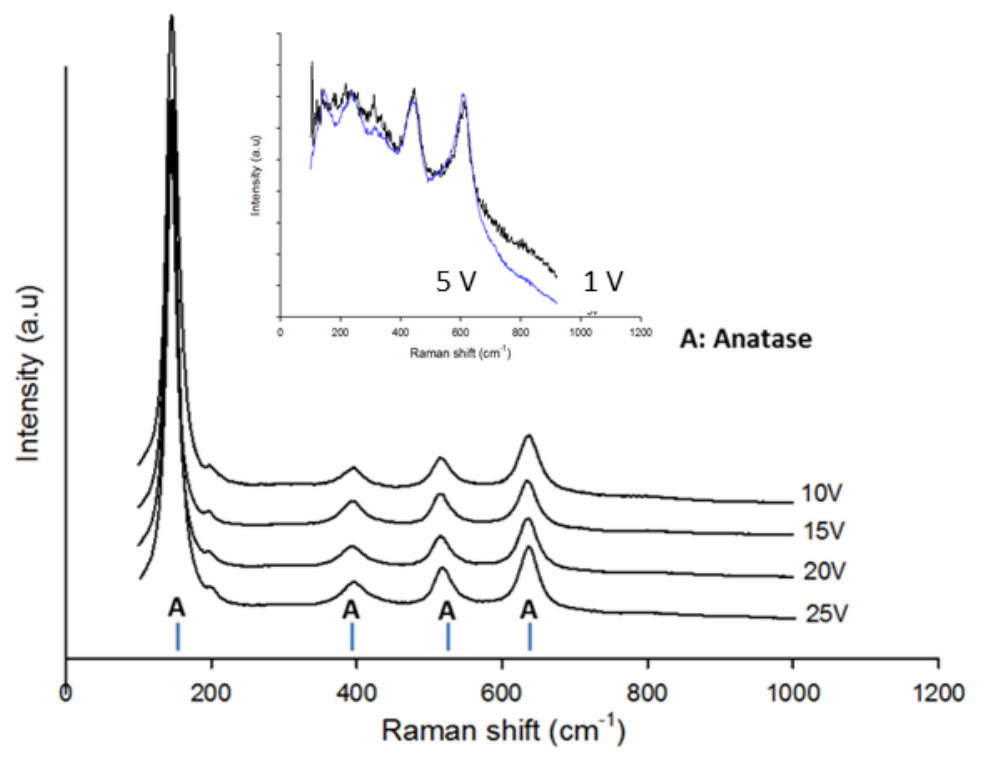

Figure S2. 5 Raman patterns of $\mathrm{TiO}_{2}$ nanotube arrays prepared under different anodization potential. All samples were annealed at $450^{\circ} \mathrm{C}$ for $30 \mathrm{~min}$

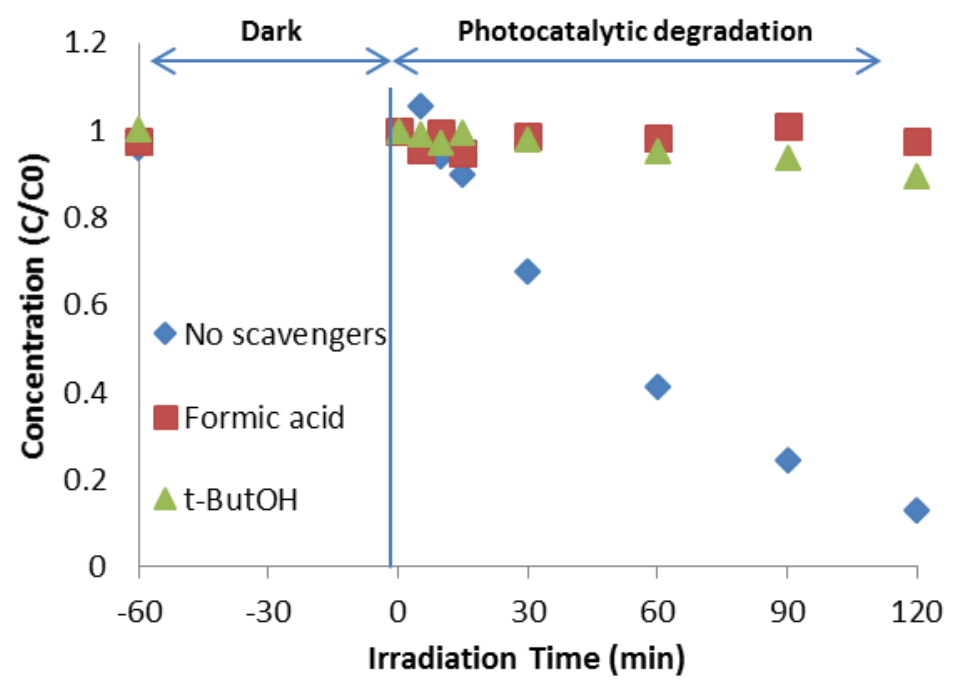

Figure S2. 6 MTP concentration profile as a function of time during photocatalytic degradation over TNAs with addition of scavengers 
Chapter 2

Table S2. 2 Degradation by-products identified during photocatalytic degradation of metoprolol by $\mathrm{TiO}_{2}$ nanotube arrays under irradiation of UV-LED

\begin{tabular}{|c|c|c|c|c|c|}
\hline Intermediates & $\mathrm{m} / \mathrm{z}$ & Retention time (min) & No scavenger & Formic acid & t-BuOH \\
\hline DP1 & 74 & 1.12 & $\mathrm{~V}$ & - & - \\
\hline DP2 & 150 & 1.14 & 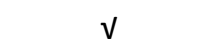 & - & - \\
\hline DP3 & 134 & 1.22 & $\sqrt{ }$ & $\sqrt{ }$ & $\sqrt{ }$ \\
\hline DP4 & 76 & 1.23 & $v$ & $v$ & $v$ \\
\hline DP5 & 102 & 1.23 & $\sqrt{ }$ & - & - \\
\hline DP6 & 120 & 1.23 & 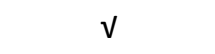 & 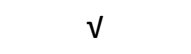 & $\sqrt{ }$ \\
\hline DP7 & 118 & 1.41 & 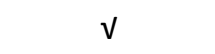 & - & - \\
\hline DP8 & 104 & 1.43 & 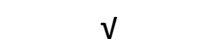 & - & - \\
\hline DP9 & 220 & 1.49 & $v$ & - & - \\
\hline DP10 & 270 & 2.23 & $v$ & - & - \\
\hline DP11 & 240 & 3.99 & 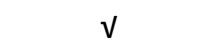 & - & - \\
\hline DP12 & 254 & 4.05 & v & - & - \\
\hline DP13 & 300 & 4.46 & $v$ & - & - \\
\hline DP14 & 270 & 4.55 & v & - & - \\
\hline DP15 & 284 & 4.66 & $v$ & - & - \\
\hline DP16 & 254 & 4.79 & $v$ & - & - \\
\hline DP17 & 316 & 4.87 & $v$ & - & - \\
\hline DP18 & 238 & 4.88 & $v$ & - & - \\
\hline DP19 & 300 & 4.96 & $v$ & - & - \\
\hline DP20 & 282 & 4.97 & v & - & - \\
\hline DP21 & 316 & 5.02 & $v$ & - & - \\
\hline DP22 & 226 & 5.12 & V & - & - \\
\hline
\end{tabular}


Chapter 2

\begin{tabular}{llllll}
\hline DP23 & 284 & 5.45 & $\mathrm{~V}$ & $\mathrm{~V}$ & $\mathrm{~V}$ \\
DP24 & 282 & 5.66 & $\mathrm{v}$ & - & $\mathrm{V}$ \\
DP25 & 314 & 5.84 & $\mathrm{v}$ & - & - \\
\hline
\end{tabular}
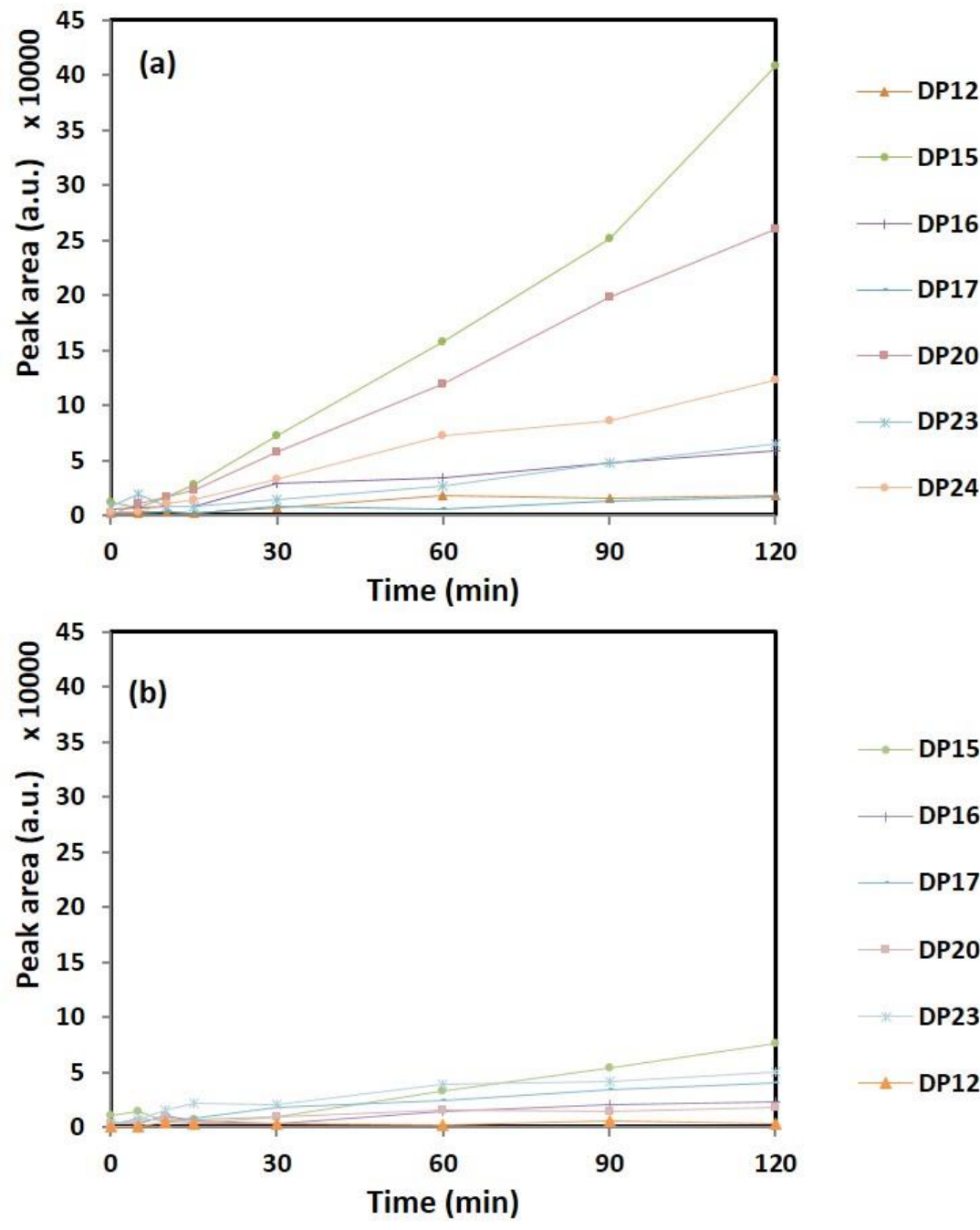

DP15 DP16 DP17

DP20

DP23

DP12

Figure S2. 7 MTP degradation products formation as a function of time during UV/TNAs treatment in the presence of specific scavenger: (a) t-BuOH; (b) formic acid. 


\section{Chapter 3}

\section{Effect of dissolved natural organic matter on} the photocatalytic micropollutant removal performance of $\mathrm{TiO}_{2}$ nanotube array

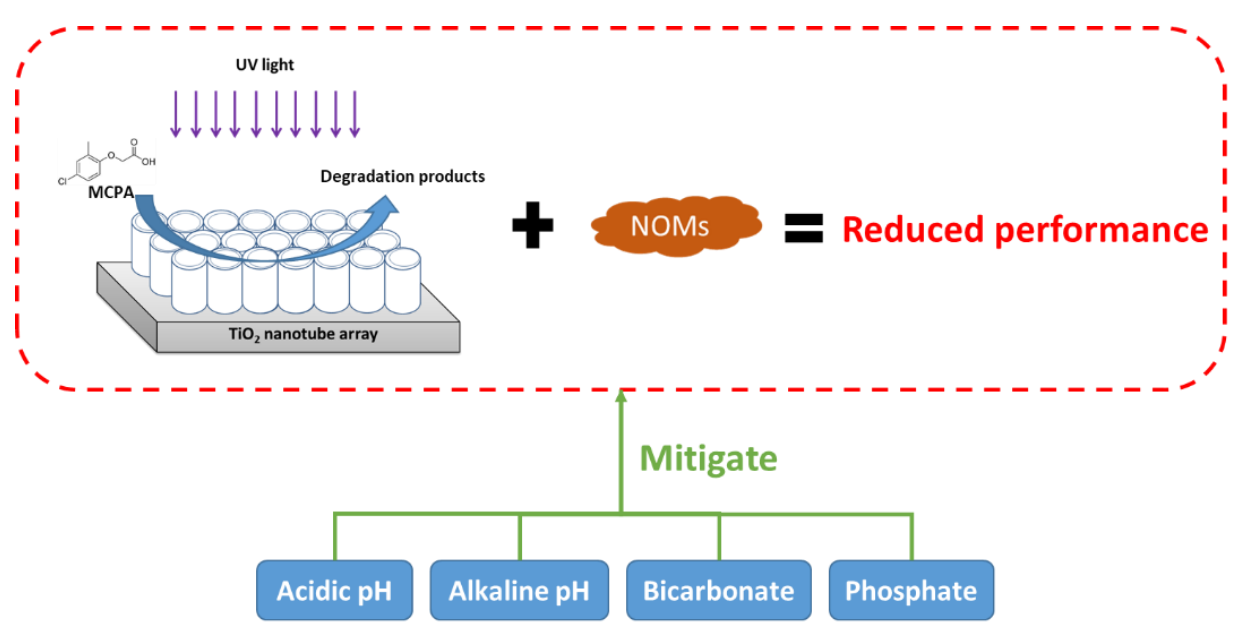

This Chapter has been submitted as:

Y. Ye, H. Bruning, W. Liu, H.H.M. Rijnaarts, D. Yntema, Effect of dissolved natural organic matter on the photocatalytic micropollutant removal performance of $\mathrm{TiO}_{2}$ nanotube array. 


\section{Chapter 3}

\section{Abstract}

The $\mathrm{TiO}_{2}$ nanotube array (TNA) is a promising photocatalyst for removal of micropollutants from water, but better understanding on its applicability in complex water matrices is still desired. Therefore this study investigates the effect of dissolved natural organic matter (NOMs) on MCPA (a typical micropollutant found in many water bodies) removal performance of TNA. The present study shows that although in bulk liquid phase NOMs would undergo photosensitization that can contribute to MCPA removal, the overall effect of NOMs on MCPA removal is detrimental due to the interaction between NOMs and the TNA surface. Acidic $\mathrm{pH}$ was found to be able to mitigate the detrimental effect of NOMs, and the photosensitization effect of NOMs was strengthened; while under alkaline $\mathrm{pH}$ conditions the detrimental effect of NOMs completely vanished. Two commonly present co-existing anions, i.e. phosphate and bicarbonate, also mitigate the detrimental effect of NOMs, and the photocurrent measurement support that the presence of such anions greatly suppresses the $\mathrm{h}+$ scavenging effect of NOMs; while other anions, i.e. chloride, nitrate, sulfate, showed no notable effect. 


\subsection{Introduction}

In recent decades, many micropollutants, including pharmaceuticals, antibiotics, herbicides, pesticides, personal care products, etc., have been frequently detected in different water bodies worldwide. Although the presence of those contaminants in the aquatic environment is usually at trace concentrations ranging from $\mathrm{ng} / \mathrm{L}$ to $\mu \mathrm{g} / \mathrm{L}[1,17,18$, 26], undesirable effects on the ecosystems are usually associated: many previous studies have pointed out that those contaminants imposes potential hazards to aquatic life at different levels, from algae to fish, even at low concentrations [27]. Vast efforts have been devoted to developing techniques for their removal, among which $\mathrm{TiO}_{2}$ photocatalysis have received increasing research interests.

The principles of $\mathrm{TiO}_{2}$ photocatalytic techniques have been described in literature $[62,103]$, which involve generation of electron/hole $\left(\mathrm{e}^{-} / \mathrm{h}^{+}\right)$pairs via excitation by photons with energy higher than the band gap energy of $\mathrm{TiO}_{2}$, followed by formation of reactive oxygen species (ROS) like hydroxyl radicals $(\cdot \mathrm{OH})$ and superoxide radicals $\left(\cdot \mathrm{O}_{2}{ }^{-}\right)$. Both the produced ROS and valence band holes $\left(\mathrm{h}^{+}\right)$contribute to micropollutant removal in $\mathrm{TiO}_{2}$ photocatalytic processes. Examples of its application in micropollutant removal are abundant in literature $[107,141,148]$.

However, it is also well documented that commonly present dissolved natural organic matter (NOM) in water bodies is a major limiting factor that imposes significant inhibitory effect on micropollutant removal performance of $\mathrm{TiO}_{2}$ photocatalytic techniques. Such inhibitory effect of NOMs can be attributed to three main facts. Firstly, the NOMs present in water matrices act as the "inner UV filter". NOMs have strong absorption in UV and near UV range, so the presence of NOMs in water would decrease the availability of UV light for $\mathrm{TiO}_{2}$ to produce ROS, thus decreasing the ROS and $\mathrm{h}^{+}$production. Secondly, NOM can also act as scavenger of $\cdot \mathrm{OH}$ and $\mathrm{h}^{+}$[177], which are known as the primary oxidants in $\mathrm{TiO}_{2}$ photocatalytic systems $[109,210]$. Thirdly, NOMs can inhibit the target pollutant degradation via competitive adsorption on the $\mathrm{TiO}_{2}$ surface [211]. The inhibitory effect of $\mathrm{NOM}$ on $\mathrm{TiO}_{2}$ based photocatalytic micropollutant removal processes has been documented in previous studies on $\mathrm{TiO}_{2}$ slurry systems. For instance, Brame et al. conducted a mechanistic study on the inhibitory effect of $\mathrm{NOMs}$ on $\mathrm{TiO}_{2}$ slurry system, and a mechanistic 


\section{Chapter 3}

model was developed which suggesting the competitive adsorption by NOMs and ROS scavenging were the most influential inhibitory mechanisms [212]. A recent work presented by Peng et al. suggested that the reactivity of $\mathrm{TiO}_{2}$ nanoparticles could change significantly after long exposure to natural water, because: (1) a NOM layer can be formed on and cap the $\mathrm{TiO}_{2}$ particles surface via adsorption; (2) the adsorbed NOM layer can act as ROS scavenger and subsequently reduce the concentration of ROS in the bulk liquid phase [143]. For $\mathrm{TiO}_{2}$ slurry systems where $\mathrm{TiO}_{2}$ nanoparticles are employed, the presence of NOMs can also interfere with the system performance by changing the stability of the $\mathrm{TiO}_{2}$ nanoparticles [213]. Focus was given to $\mathrm{TiO}_{2}$ slurry systems, where $\mathrm{TiO}_{2}$ nanoparticles are used, in previous studies on the effect of NOM [214], but regarding real life applications immobilization of $\mathrm{TiO}_{2}$ should be considered to achieve better retention and reuse of the catalyst. The electrochemical anodic produced $\mathrm{TiO}_{2}$ nanotube array (TNA) is a promising option because of its multiple merits: (1) large surface area; (2) high stability; (3) oriented electron transport which can reducing $\mathrm{e}^{-/ \mathrm{h}^{+}}$pairs recombination; (4) relatively easy to make and (5) tunable morphologies. Examples of using of TNAs for organic pollutants elimination are available in literature [161-164, 210]. The change in $\mathrm{TiO}_{2}$ morphology may have an impact on the effect of NOM, but very little is known about the effect of NOM on TNA based photocatalytic system.

In practice, the presence of NOM in water bodies is associated with the presence of inorganics. For example, phosphate, sulfate, bicarbonate, chloride, etc., are the most commonly present inorganic anion species in a broad range of water matrices. The photocatalytic removal of micropollutants by $\mathrm{TiO}_{2}$ photocatalytic processes can also be affected by those co-existing inorganic anions by competitive adsorption and interaction with $\operatorname{ROS}[168,174,215]$. In this context, the presence of co-existing inorganic anions may impose impact on the effect of NOMs on a photocatalytic system. In a recent study by Long et al., the change in the detrimental effect of humic acids on photocatalytic performance of $\mathrm{TiO}_{2}$ particles by the presence of phosphate was reported [142]. However, the combined effects of NOMs and other commonly present inorganic anions have not been well documented in literature. 
Given this context, the present work aims to study the effect of NOM on the photocatalytic micropollutant removal performance of TNA. The model micropollutant compound employed in this study is 4-chloro-2-methylphenoxyacetic acid (MCPA), which is a frequently encountered pollutant in different water bodies including ground water and tap water sources $[216,217]$, and its toxicity and endocrine disrupting effects on living species has been abundantly evidenced in literature $[218,219]$. The effect of NOM on MCPA removal during photocatalytic degradation by TNA is investigated. The effect of $\mathrm{pH}$ and the effects of co-existing common inorganic anions are documented.

\subsection{Experimental methods}

\subsubsection{Materials}

Titanium foil ( $\geq 99.5 \%, 0.3 \mathrm{~mm}$ thick) was purchased from the Titaniumshop (The Netherlands). Ammonium sulphate ( $\geq 99.0 \%)$, ammonium fluoride $(\geq 98.0 \%)$, tert-Butanol ( $\geq 99.0 \%)$, formic acid ( $\geq 96 \%$ ) were obtained from Sigma-Aldrich (Germany). MCPA ( $\geq 98.0 \%$ ) was purchased from Dr Ehrenstorfer GmbH (Germany). All chemicals were used without further purification. Stock solution of MCPA was prepared at concentration of $200 \mathrm{mg} / \mathrm{L}$. Sodium bicarbonate ( $\geq 99.9 \%)$, Hydrochloric acid (1 mol/L), sodium sulphate ( $\geq 99.0 \%$ ), sodium chloride (100\%), sodium nitrate ( $\geq 99.0 \%)$, tri-sodium phosphate dodecahydrate $(\geq 98.0 \%)$ and sodium hydroxide aqueous solution ( $1 \mathrm{~mol} / \mathrm{L})$ were purchased from VWR (Belgium) and was used as received. Aquatic NOM Suwannee River NOM (SWR-NOM) (2R101N) and Upper Mississippi River NOM (UMR-NOM) (1R110N) were obtained from International Humic Substances Society (IHSS) and received as dry solid extracts. Stock solutions of the NOMs were prepared at concentration of $100 \mathrm{mg} / \mathrm{L}$. Ultrapure water from a Milli-Q Advantage A10 system (Merck Millipore, Darmstadt, Germany) was used for preparation of stock solutions and reaction solutions.

\subsubsection{Fabrication and characterization of the $\mathrm{TiO}_{2}$ nanotube array}

The TNA employed in this study was prepared with the electrochemical anodization method we previously reported [210]. The titanium foil was first degreased by successively ultra-sonication for 15 min in 2-propanol, acetone and Milli-Q water, and then dried in $\mathrm{N}_{2}$ atmosphere, as pretreatment. The electrochemical anodization was performed 


\section{Chapter 3}

in a two-electrode chemical cell connected to an EST150 DC power supply (Delta Elektronika, The Netherlands). The titanium foil with a size of $3 \mathrm{~cm} \times 4.5 \mathrm{~cm}$ was used as anode, and another titanium foil with a size of $4.5 \mathrm{~cm} \times 5 \mathrm{~cm}$ was used as cathode. The interval between the electrodes was $2 \mathrm{~cm}$. The titanium foil was anodized in $100 \mathrm{~mL}$ aqueous electrolyte $(0.15$

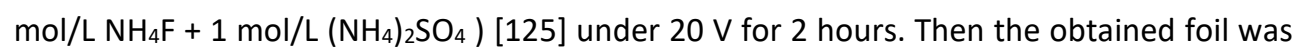
first rinsed and then further sonication washed with Milli-Q water to remove residue electrolyte and impurities on the surface. Subsequently, the treated Ti foil was annealed at $600{ }^{\circ} \mathrm{C}$ for $0.5 \mathrm{~h}$ in a furnace (Nabertherm, Germany) in air atmosphere and was cooled gradually back to ambient temperature after annealing. The surface morphologies of the TNAs electrode were characterized by scanning electron microscopy (SEM) and the chemical composition of the prepared TNAs was measured by EDX. The SEM/EDX analyses were performed using a JEOL JSM 6480 scanning electron microscope (JEOL Europe B.V., Nieuw-Vennep, The Netherlands) equipped with a EDX system. Raman spectra of the prepared TNA were recorded using a Horiba LabRAM spectrometer equipped with a mpc3000 laser (532.2 nm), an $800 \mathrm{~mm}$ focal length achromatic flat field monochromator (grating of 600 grooves $/ \mathrm{mm}$ ) and a Synapse multichannel air cooled $\left(-70^{\circ} \mathrm{C}\right) \mathrm{CCD}$ detector.

\subsubsection{Photocatalytic MCPA degradation experiments}

The photocatalytic experiments were conducted in a crystallizing dish with a 450 mW UV-LED light source module placed on the top. The UV-LED light source module consisted of a UV-LED (NCSU033B, NICHIA, Japan) and has peak emission wavelength of 365 $\mathrm{nm}$, and an aluminium plate served as heat dispenser. A schematic of the set-up is shown in Figure 3. 1. 


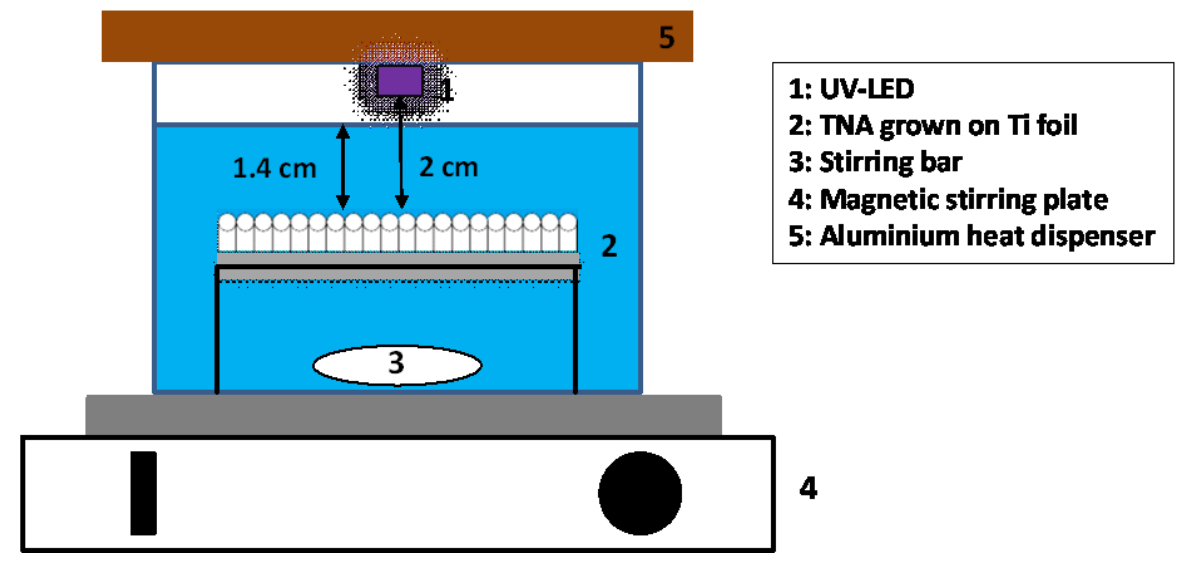

Figure 3. 1 Schematic representation of the experimental set-up

The photocatalytic MCPA degradation experiments were carried out with a reaction solution volume of $60 \mathrm{~mL}$, with the presence of TNA (size of $3 \mathrm{~cm} \times 3 \mathrm{~cm}$ ), at ambient temperature. The distance between the UV-LED and the surface of TNAs was $2 \mathrm{~cm}$, and the radiant power of the UV-LED at this distance was $18.6 \mathrm{~mW} / \mathrm{cm}^{2}$ measured by a THORLABS S150C radiant power meter (THORLABS, USA). Vigorous mixing was applied as soon as the reaction solution was added into the reactor. Before switching on the UV-LED, the reaction system was kept in dark for 1 hour to establish equilibrium of any possible adsorption of MCPA on the TNA surface. Then the photocatalytic experiments started, for the duration of $120 \mathrm{~min}$. At designated time intervals $1 \mathrm{~mL}$ samples were taken, and stored in dark at $4{ }^{\circ} \mathrm{C}$ till LC-MS/MS analysis. All experiments were carried out in duplicate. Except for experiments conducted in designated acidic or alkaline conditions, all other experiments were conducted with natural initial pH (circum-neutral: 6-7) and without $\mathrm{pH}$ adjustment. For experiments conducted in designated acidic or alkaline conditions, hydrochloric acid or sodium hydroxide aqueous solution were applied to adjust the $\mathrm{pH}$.

\subsubsection{MCPA concentration measurement procedures}

For MCPA concentration measurement, an Agilent LC-MS/MS system consisting of Agilent infinity 1260 LC-system (degasser, binary pump, auto sampler with cooled tray and column oven) and Agilent 6420 triple Quadrupole Mass Spectrometer with Electrospray ion 


\section{Chapter 3}

source was used. Detailed information of the analytical method used can be found in literature [220].

\subsection{Results and discussion}

\subsubsection{Characterization of the $\mathrm{TiO}_{2}$ nanotube array}

Figure 3. 2a shows the SEM top view image of the TNA, demonstrating the selforganised tubular structure layer, which has average pore size of around $80 \mathrm{~nm}$. The Raman spectrum of the TNA surface shown in Figure 3. $2 \mathrm{~b}$ demonstrates that the TNA layer has a crystallographic phase consisting of both anatase and rutile. Good photocatalytic performance of $\mathrm{TiO}_{2}$ contains both anatase and rutile has been documented in literature $[203,210]$.
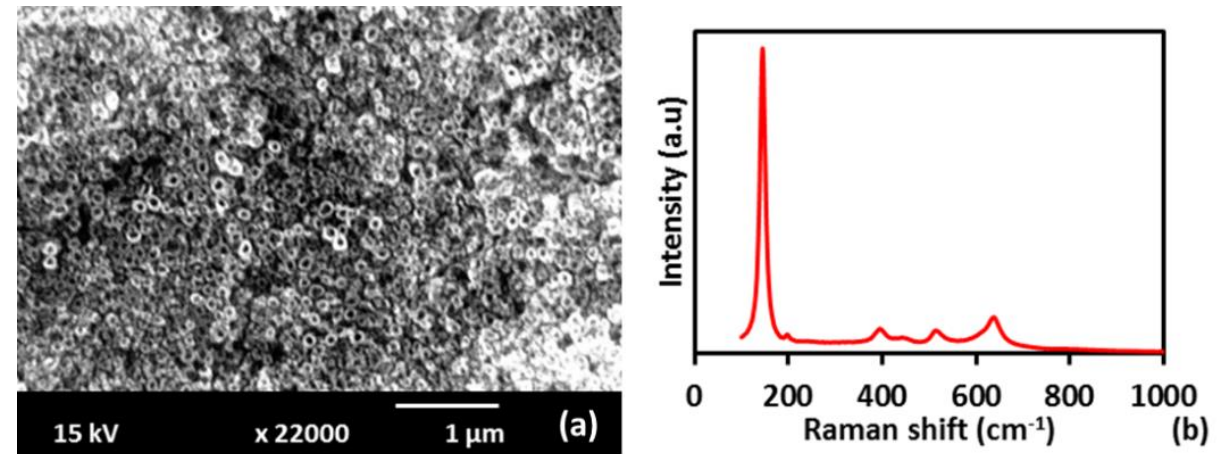

Figure 3. 2 Surface morphology (a) and the Raman spectrum (b) of the $\mathrm{TiO}_{2}$ nanotube array

\subsubsection{Effect of NOM on photocatalytic MCPA removal performance of TNA}

Experiments were carried out in the presence of two different types NOMs originating from two large rivers at varied concentrations ranging from $5 \mathrm{mg} / \mathrm{L}$ to $15 \mathrm{mg} / \mathrm{L}$, to examine the effect of NOMs on photocatalytic MCPA removal performance of TNA. 


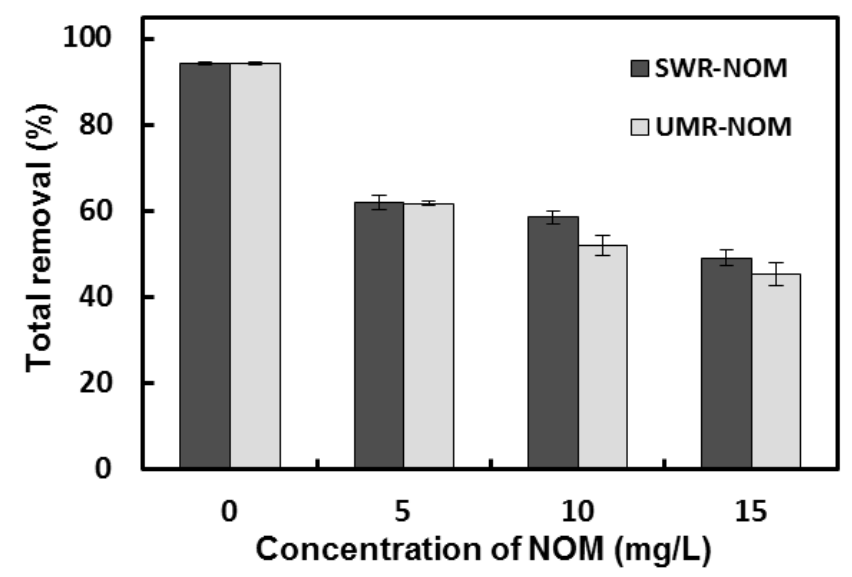

Figure 3. 3 Effect of NOMs on the photocatalytic MCPA removal performance of TNA. Conditions: natural pH ( 7), $[\mathrm{MCPA}]_{0}=1 \mathrm{mg} / \mathrm{L}, 120 \mathrm{~min}$ treatment.

The results (see Figure 3. 3) show that the presences of both NOMs impose significant detrimental effect on the removal of MCPA over TNA. The TNA based photocatalytic process enabled rather rapid MCPA degradation with a total removal of $94.3 \%$ after 120 min treatment in absence of NOMs. The presence of $5 \mathrm{mg} / \mathrm{L}$ NOM resulted in a drastic decrease in MCPA removal: the total removal of MCPA decreased to only $62.0 \%$ and $61.8 \%$, in the presence of SWR-NOM and UMR-NOM respectively. Increasing NOM concentrations led to a further decrease in MCPA removal. With elevated concentration of SWR-NOM to $10 \mathrm{mg} / \mathrm{L}$ and $15 \mathrm{mg} / \mathrm{L}$, the total removal of MCPA decreased to $58.5 \%$ and 49.1\%. For UMR-NOM, when its concentration increased to $10 \mathrm{mg} / \mathrm{L}$ and $15 \mathrm{mg} / \mathrm{L}$, the total removal of MCPA reduced to $52.0 \%$ and $45.2 \%$. It is also found that the two tested NOMs exert the same inhibitory effect on MCPA removal performance of the TNA, and no significant difference between the two NOMs can be found.

It should be noticed that, upon absorbing UV photons, NOMs can also initiate photosensitized degradation of organic pollutants $[178,180]$. Experiments were conducted to examine this photosensitization effect of NOMs, and the results (Figure 3. 4) show that the presence of SWR-NOM and UMR-NOM can indeed contribute to MCPA removal in the bulk liquid phase. However, the negative effect of both NOMs outweighs the 


\section{Chapter 3}

photosensitization effect; making the presence of NOMs exhibits detrimental effect on MCPA removal in the TNA based photocatalytic system.

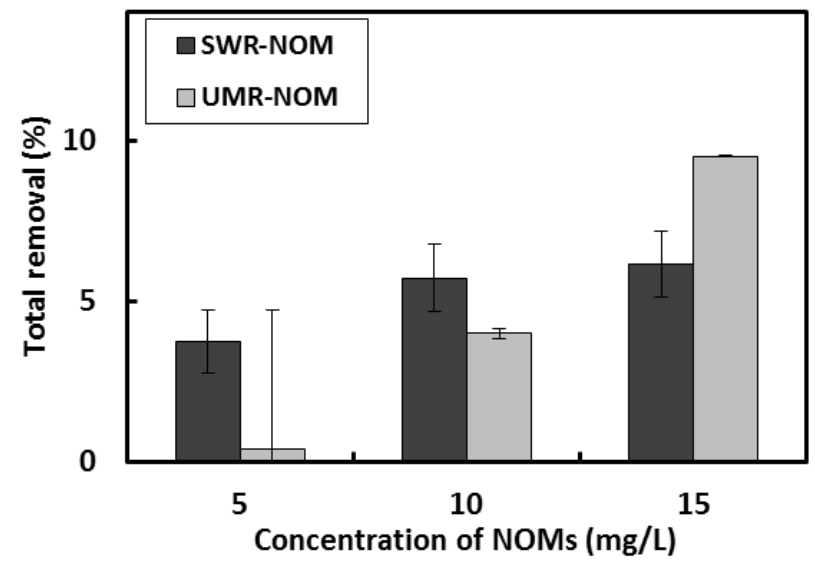

Figure 3. 4 Photosensitization effect of NOMs on MCPA removal. Conditions: natural pH ( 7), $[\mathrm{MCPA}]_{0}=1 \mathrm{mg} / \mathrm{L}, 120 \mathrm{~min}$ treatment, no TNA.

\subsubsection{The $\mathrm{pH}$ influences the effect of NOMs}

Previous studies have pointed out that the $\mathrm{pH}$ conditions impose impact on the adsorption of $\mathrm{NOMs}_{\text {on }} \mathrm{TiO}_{2}$ surfaces $[211,221]$. Considering the adsorption of NOMs on the catalysts surface is a crucial factor contributing to its inhibitory effect, it is of importance to elucidate the effect of $\mathrm{pH}$ on the detrimental effect of NOM on the micropollutant removal performance of TNA. Therefore, experiments were conducted to examine the effects of NOMs at varied concentrations under different $\mathrm{pH}$ conditions on MCPA removal over TNA. 

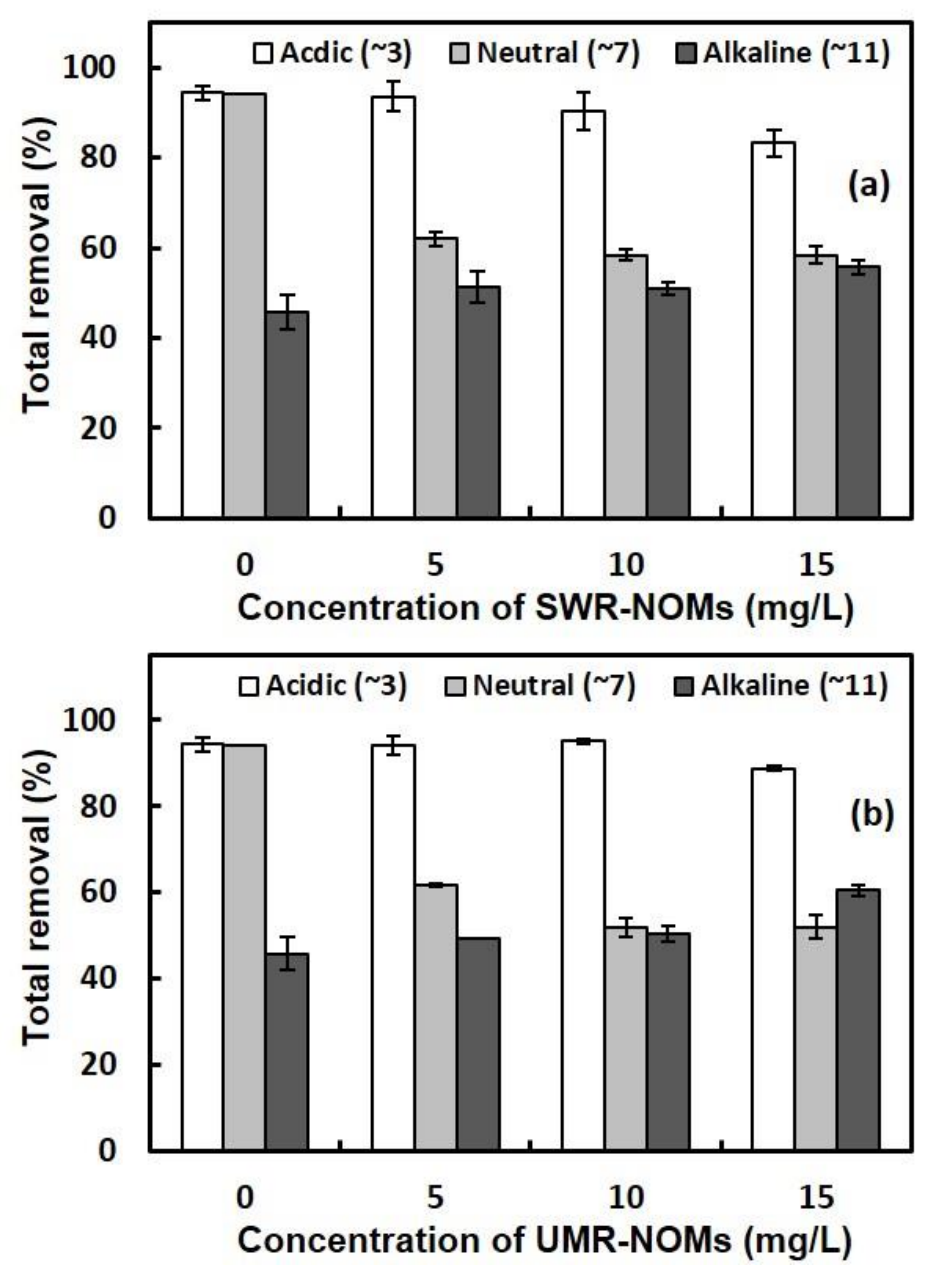

Figure 3. 5 Effects of NOMs on the photocatalytic MCPA removal performance of TNA, under different $\mathrm{pH}$ conditions

The results (Figure 3.5 ) suggest that the change in $\mathrm{pH}$ conditions would change the effect of NOMs significantly. At neutral pH, the presence of even low level of NOMs led to drastic decrease in MCPA removal. Although the alkaline $\mathrm{pH}$ itself induced a decrease in the removal target pollutant (as documented in our previous study [210]), the inhibitory effects of NOMs were not observed under alkaline conditions. On the contrary, a slight increase in the MCPA removal with increasing NOM concentration was observed. This finding can be attributed to two facts: (1) the alkaline conditions mitigate the competitive adsorption by NOMs [211, 221]; and (2) the NOMs still impose photosensitization effect which can 


\section{Chapter 3}

contribute to MCPA removal in the liquid phase (Figure 3. 6). More interestingly, although many previous studies have documented that the acidic $\mathrm{pH}$ would benefit the adsorption of NOMs [211,221], only minor inhibitory effects were observed in the presence of NOMs at high concentration level $(15 \mathrm{mg} / \mathrm{L}$ ) under acidic conditions. This can be attributed to strengthened adsorption of MCPA [115] on TNA surface and therefore strengthened oxidation by valence band holes and surface adsorbed hydroxyl radicals, as well as largely strengthened photosensitization effect of NOMs (Figure 3. 6).
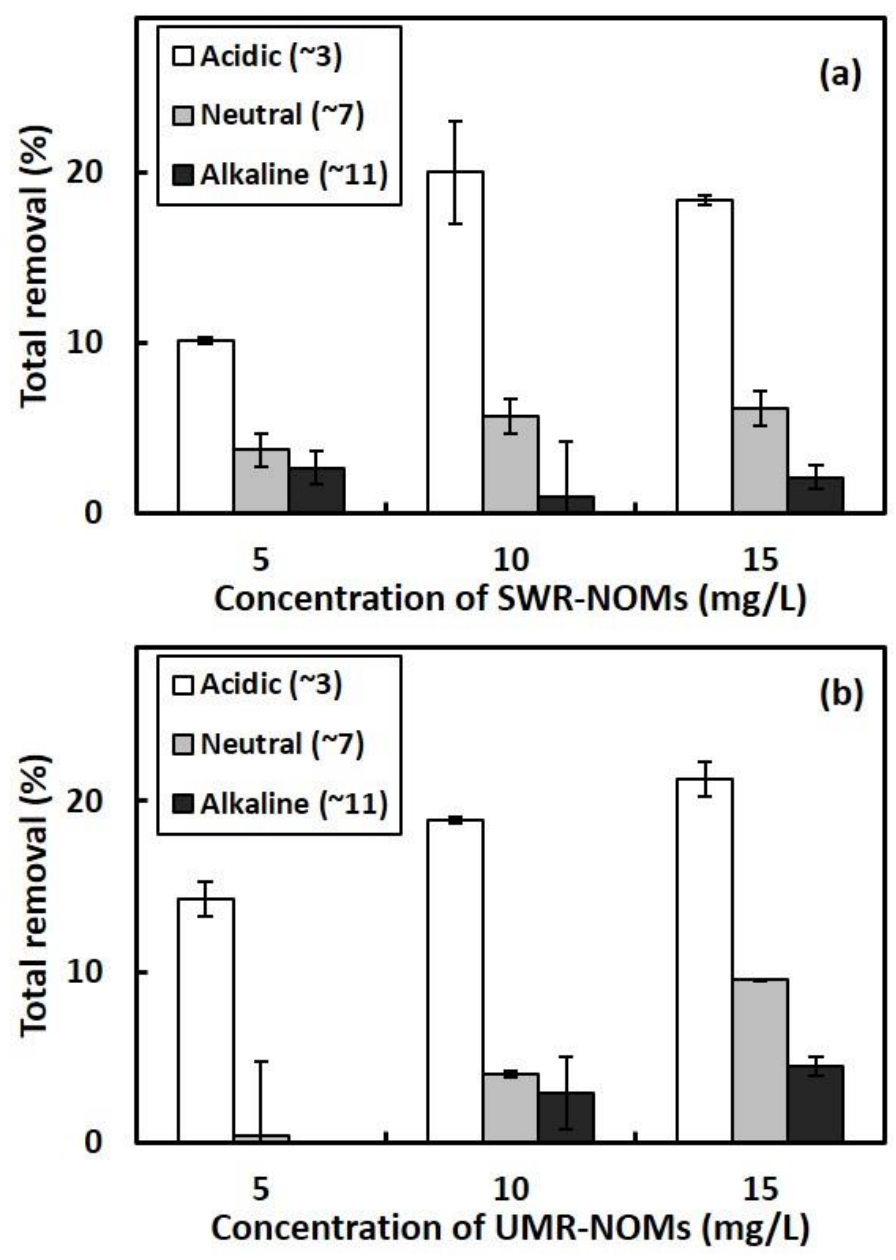

Figure 3. 6 Photosensitization effect of NOMs on MCPA removal at different pH conditions. Conditions: $[\mathrm{MCPA}]_{0}=1 \mathrm{mg} / \mathrm{L}, 120 \mathrm{~min}$ treatment, no TNA. 


\subsubsection{Role of co-existing inorganic anion species}

To study the impact of co-existing inorganic anions on NOM's inhibitory effect, experiments were performed to examine the photocatalytic MCPA removal performance at various NOMs concentrations with the presence of some most common inorganic anion species, i.e. phosphate, bicarbonate, sulfate, nitrate, chloride.

The results (see Figure 3. 7 and Figure 3. 8) suggest that both phosphate and bicarbonate can mitigate the inhibitory effect of NOMs. Without the presence of inorganic anions, the MCPA removal lowered from $94.3 \%$ to $45.2 \%$ and $49.1 \%$, with the presence of $15 \mathrm{mg} / \mathrm{L}$ UMR-NOM and SWR-NOM respectively. In the presence of $50 \mathrm{mg} / \mathrm{L}$ phosphate anions, even at highest NOMs concentration $(15 \mathrm{mg} / \mathrm{L})$, the inhibitory effects of NOMs were significantly reduced: $73.1 \%$ (in presence of UMR-NOM) and 62.5\% (presence of SWR-NOM) MCPA removal was achieved within the same treatment time span. For bicarbonate, such mitigation on NOMs' inhibitory effects was also observed, but at weaker level (Figure 3. 8). With the presence of $100 \mathrm{mg} / \mathrm{L}$ bicarbonate, $73.1 \%$ and 62.5\% MCPA removal was achieved with the presence of $15 \mathrm{mg} / \mathrm{L}$ SWR-NOM and UMR-NOM, respectively.

A similar phenomenon has been reported in $\mathrm{TiO}_{2}$ slurry system in a recent study conducted by Long et al. [142]. The authors observed that phosphate counteracts the inhibitory effect of humic acids (an important constituent of $\mathrm{NOM}$ ) on $\mathrm{TiO}_{2}$ nanoparticles based system, and attributed such phenomenon to reduced humic acid adsorption (and therefore direct ROS scavenging upon adsorption) and enhanced electron transfer via changing the adsorption site of humic acid, by the presence of phosphate. Moreover, in another study conducted by Zhao et al. [209] on phosphate surface modification of $\mathrm{TiO}_{2}$ nanoparticles, it was found that the adsorption of phosphate ions on the catalyst surface would lead to enhanced photocatalytic degradation of target pollutants by promoting the separation of $\mathrm{e}^{-} / \mathrm{h}^{+}$pairs via introducing an electrostatic field on the catalyst surface. 

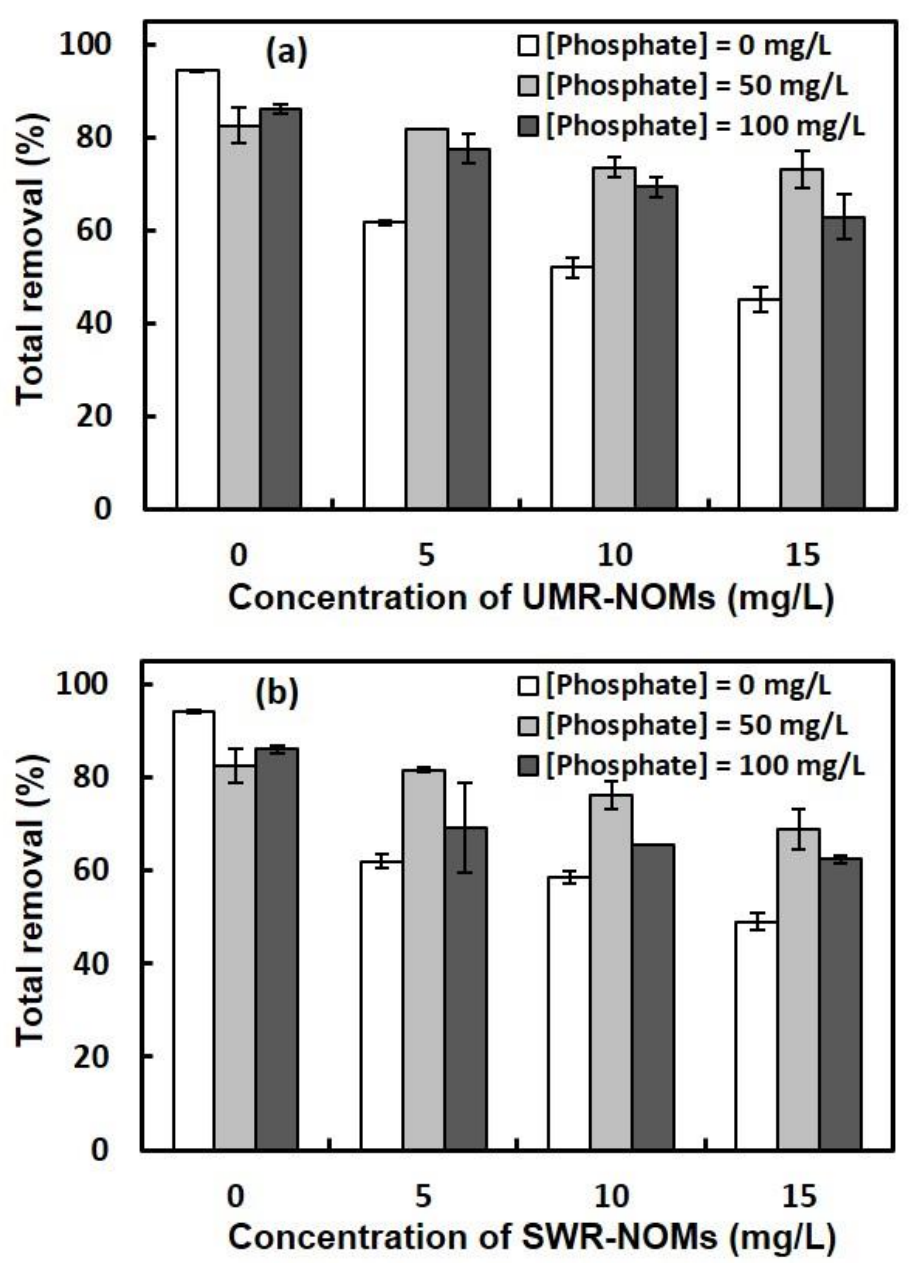

Figure 3. 7 Effects of NOMs on the photocatalytic MCPA removal performance of TNA, with the presence of phosphate anions. Conditions: natural pH ( 7), $[\mathrm{MCPA}]_{0}=1 \mathrm{mg} / \mathrm{L}$, 120 min treatment. 

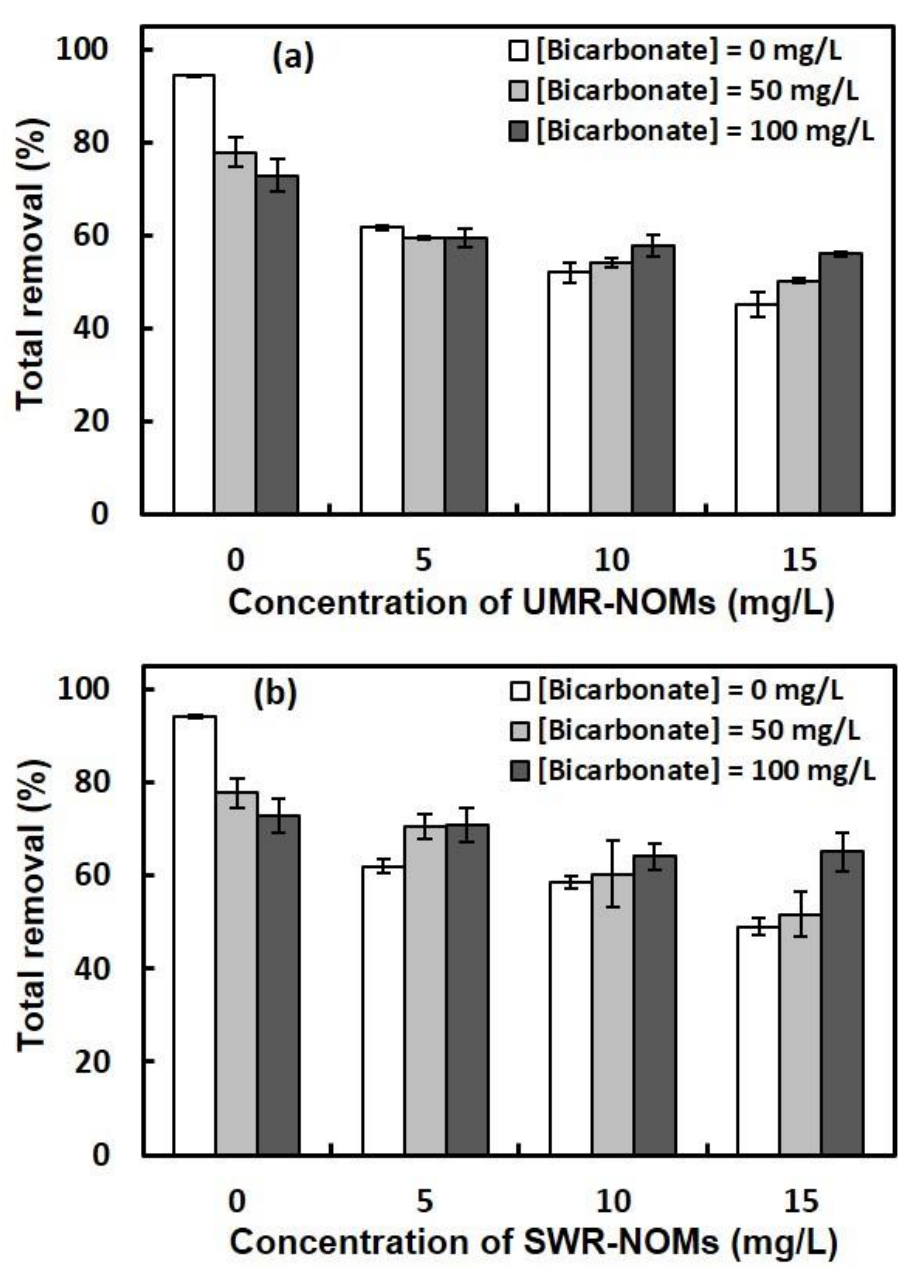

Figure 3. 8 Effects of NOMs on the photocatalytic MCPA removal performance of TNA, with the presence of bicarbonate anions. Conditions: natural pH ( 7), $[\mathrm{MCPA}]_{0}=1 \mathrm{mg} / \mathrm{L}$, 120 min treatment.

The effect of competitive adsorption between phosphate anions and NOMs was investigated using a transient photo-electrolysis technique and described by a doubleexponential model [222] (details of this method is described in Text S3.1). The timedependent photocurrent curves are shown in Figure 3. 9b. Upon UV irradiation, the photocurrent increased to a peak value and would then decay gradually to a steady state. The decay curves $(5 s-10 s)$ can fits well with a double-exponential model $\left(R^{2}>0.99\right)$, where the two exponential decay terms reveal both surface and interfacial photocatalytic 


\section{Chapter 3}

reactions (in this case, NOM oxidation). The relevant rate constants were calculated and shown in Table 3. 1. The obtained results suggest that the presence of phosphate anions can indeed reduce the NOM oxidation on TNA surface.

For bicarbonate, its role observed in the present work is rather different from a previously reported paper on $\mathrm{TiO}_{2}$ slurry systems. It is reported that the alkalinity impose significant inhibitory effects on the performance of $\mathrm{TiO}_{2}$ slurry system mainly due to formation of larger $\mathrm{TiO}_{2}$ aggregates [174]. However, such mechanism can be neglected in the present work because TNA, instead of $\mathrm{TiO}_{2}$ nanoparticles, was employed. In addition, quenching of conduction band electrons by bicarbonate can be a major factor that contributes to the mitigation effect of their presence on the NOMs` inhibitory effect. According to our previous study [210], bicarbonate anions can act as conduction band electrons quencher [208] upon adsorption on the $\mathrm{TiO}_{2}$ surface, which decreases the recombination of $\mathrm{e}^{-} / \mathrm{h}^{+}$pairs and can in turn enhance the generation of ROS. In addition, competitive adsorption of bicarbonate against NOMs was also observed, which can reduce the $\mathrm{h}^{+}$scavenging effect of NOMs (Figure 3. 1b). The photocurrent tests results support that the presence of bicarbonate anions can greatly reduce the surface related NOM oxidation: the surface oxidation related photocurrent decay rate constants were much lower with the presence of bicarbonate (Table 3. 1). 

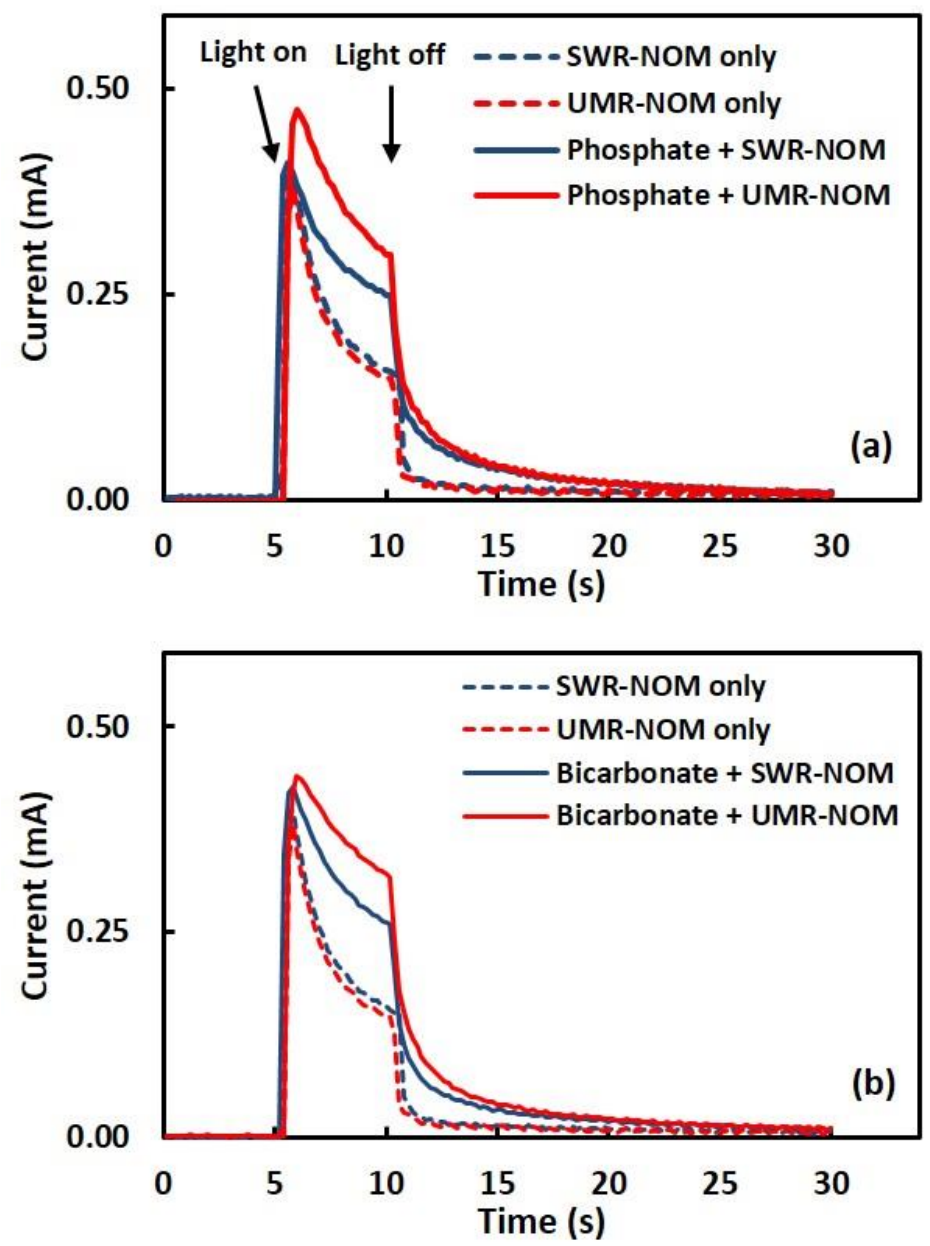

Figure 3. 9 Time dependent photocurrent generation by TNA. Conditions: $0.1 \mathrm{M} \mathrm{NaClO}_{4}$ electrolyte, $[\mathrm{NOM}]=15 \mathrm{mg} / \mathrm{L}$, [Anion] $=100 \mathrm{mg} / \mathrm{L}$. 
Chapter 3

Table 3. 1 Surface processes related photocurrent decay rate constants under different conditions

\begin{tabular}{cc}
\hline Conditions & $\mathrm{ks}\left(\mathrm{s}^{-1}\right)$ \\
\hline SWR-NOM only & 0.99 \\
UMR-NOM only & 1.00 \\
Phosphate + SWR-NOM & 0.70 \\
Phosphate + UMR-NOM & 0.31 \\
Bicarbonate + SWR-NOM & 0.65 \\
Bicarbonate + UMR-NOM & 0.26 \\
\hline
\end{tabular}

Unlike phosphate and bicarbonate, the presence of sulfate, nitrate, and chloride anions imposes no mitigation effect on NOMs` inhibitory effects (Figure S3. 2, Figure S3. 3 and Figure S3. 4). This is due to the fact that these anion species can likely enhance the adsorption of NOMs (especially the humic acid components) on the catalyst [223].

\subsection{Conclusions}

In the present work, we demonstrate the effects of two aquatic NOMs collected from large rivers, i.e. SWR-NOM and UMR-NOM, on the photocatalytic micropollutant removal performance of TNA. For the micropollutant tested herein, main findings can be concluded as below:

* Both types of tested NOMs impose inhibitory effect on MCPA removal performance of TNA, even at low concentration level;

* Acidic pH counteracts the inhibitory effect of NOM, because of enhanced interaction between MCPA and the TNA surface, and strengthened photosensitization effect of NOMs; Under alkaline conditions, NOMs impose a slightly positive impact on MCPA removal, due to photosensitization effect of NOMs and mitigated competitive adsorption of NOMs; 
* The presence of a certain level of phosphate and bicarbonate anions can mitigate the inhibitory effect of NOM, while the presence sulfate, nitrate and chloride does not have such mitigation effect.

* The transient photo-electrolysis measurement results demonstrate that the presence of bicarbonate and phosphate anions can reduce the $\mathrm{h}^{+}$scavenging effect of NOMs.

\subsection{Acknowledgements}

This work was performed in the cooperation framework of Wetsus, European Centre of Excellence for Sustainable Water Technology (www.wetsus.eu). Wetsus is cofunded by the Dutch Ministry of Economic Affairs and Ministry of Infrastructure and Environment, the Province of Fryslân, and the Northern Netherlands Provinces. The authors would like to thank the participants of the research theme "Advanced water treatment" for the fruitful discussions and their financial support. The authors gratefully thank Ton van der Zande and Mieke Kersaan-Haan for the realization of the instrumental analysis. 


\section{Chapter 3}

\section{Supplementary information}

\section{Text S3.1}

The transient photoelectrolysis measurement method in this study was adopted and modified from the transient photoelectrolysis technique previously developed by Jiang et al. [222]. The applied photoelectrolysis technique is aimed to study the photocatalytic oxidation kinetics of adsorbed organic compounds on $\mathrm{TiO}_{2}$ electrodes surfaces. In the present study, such measurement was performed in a two-electrode cell (Figure S3. 1), where the TNA electrode $(3 \mathrm{~cm} \times 3 \mathrm{~cm})$ was used as photoactive anode and a copper foil (Grade M2, $3 \mathrm{~cm} \times 4.5 \mathrm{~cm}$ ) was used as counter electrode and a rectangular quartz cube was used as reaction cell $(6 \mathrm{~cm} \times 6 \mathrm{~cm} \times 6 \mathrm{~cm})$. The distance between the two electrodes was set to be approx. $2 \mathrm{~cm}$. The radiant power of UV light that reached the surface of TNAs was $11.6 \mathrm{~mW} / \mathrm{cm}^{2}$. The transient photocurrent was recorded by a Vertex potentiostat (Vertex One, Ivium Technologies B.V, The Netherlands).

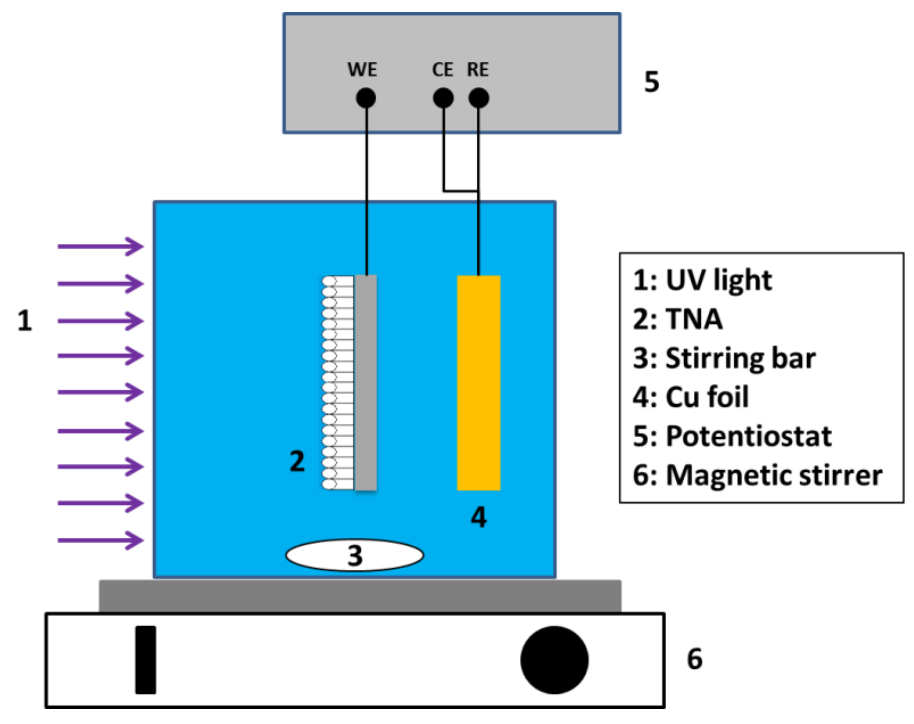

Figure S3. 1 The transient photoelectrolysis measurement set-up

Upon UV irradiation, the photocurrent increased to a peak value and would then decay gradually to a steady state. The decay curves can fit well with a double-exponential model (Equation S 3.1), where $I_{p h}$ refers to the measured photocurrent, $I_{0}$ is a constant that 
can be assigned to photocatalytic oxidation of water [142], t refers to the time lapsed from the beginning of photocurrent decay.

$$
I_{p h}=I_{0}+A e^{-k_{s} t}+B e^{-k_{i} t}
$$

The two exponential decay terms reveal both (fast) surface and (slow) interfacial photocatalytic organic compounds oxidation on the TNA, whose rates can be revealed by ks and $\mathrm{k}_{\mathrm{i}}$, respectively.
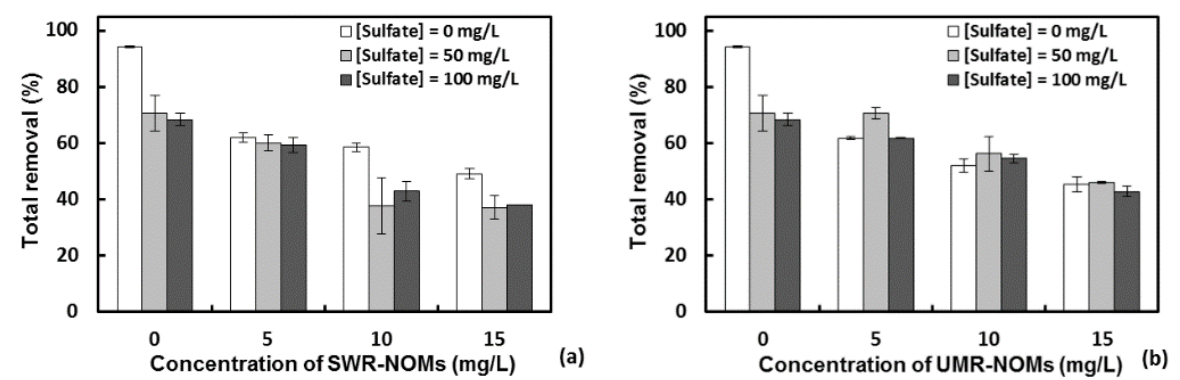

Figure S3. 2 Effects of NOMs on the photocatalytic MCPA removal performance of TNA, with the presence of sulfate anions. Conditions: natural pH ( 7), $[\mathrm{MCPA}]_{0}=1 \mathrm{mg} / \mathrm{L}, 120$ min treatment.
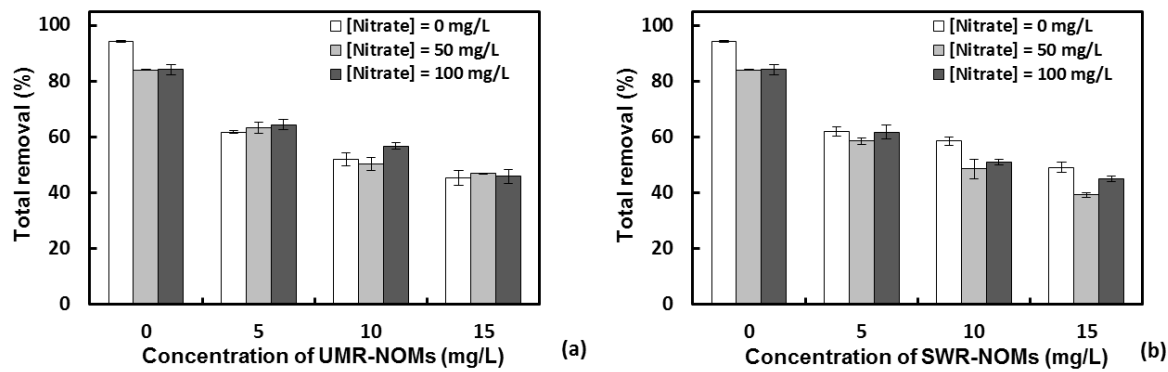

Figure S3. 3 Effects of NOMs on the photocatalytic MCPA removal performance of TNA, with the presence of nitrate anions. Conditions: natural pH ( 7), $[\mathrm{MCPA}]_{0}=1 \mathrm{mg} / \mathrm{L}, 120$ min treatment. 
Chapter 3
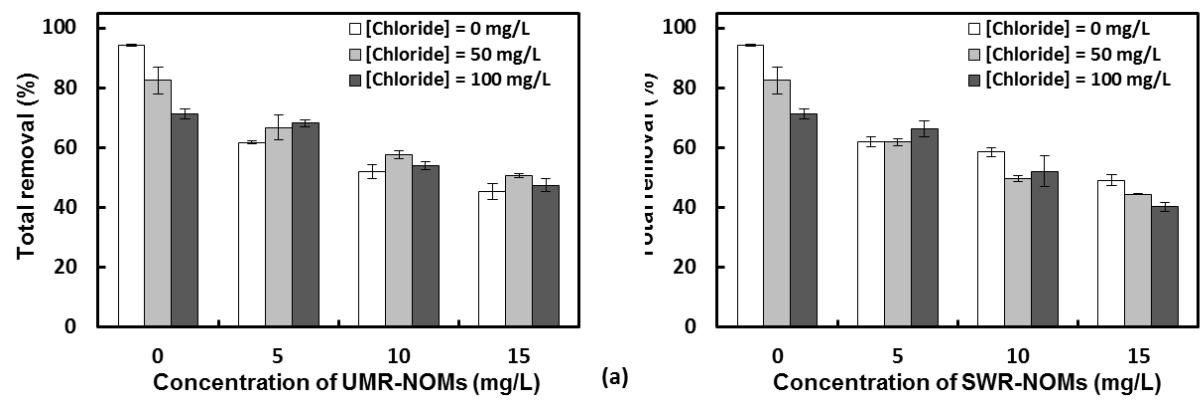

(b)

Figure S3. 4 Effects of NOMs on the photocatalytic MCPA removal performance of TNA, with the presence of chloride anions. Conditions: natural pH ( 7), [MCPA] $]_{0} 1 \mathrm{mg} / \mathrm{L}, 120$ min treatment. 


\section{Chapter 4}

\section{Significant enhancement of micropollutant photocatalytic degradation using a $\mathrm{TiO}_{2}$}

\section{nanotube array photoanode based}

\section{photocatalytic fuel cell}

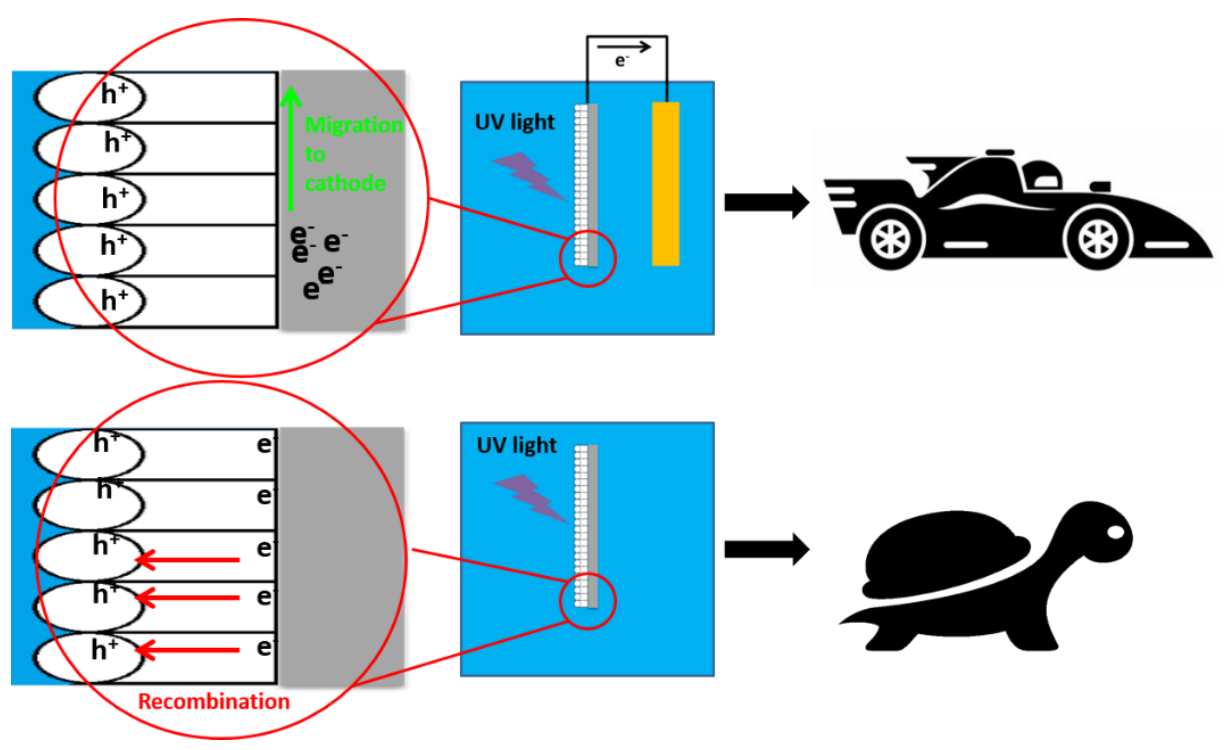

This Chapter has been published as:

Y. Ye, H. Bruning, X. Li, D. Yntema, H.H.M. Rijnaarts, Significant enhancement of micropollutant photocatalytic degradation using a TiO2 nanotube array photoanode based photocatalytic fuel cell, Chemical Engineering Journal 354 (2018) pp. 553-562 


\section{Chapter 4}

\section{Abstract}

This study evaluated the application of a membrane-free photocatalytic fuel cell composed of $\mathrm{a} \mathrm{TiO}_{2}$ nanotube array photoanode and a $\mathrm{Cu}$ cathode, i.e. TNA-Cu PFC system, for micropollutant removal from water. Significantly enhanced removal of a commonly present aqueous micropollutant 4-chloro-2-methylphenoxyacetic acid (MCPA) was obtained in this TNA-Cu PFC system: the TNA-Cu PFC system achieved better MCPA degradation compared to the conventional photocatalytic method using the same catalyst. In the TNA-Cu PFC system, the MCPA degradation was largely promoted under acidic conditions, indicating this as an important operational condition. The enhancement of MCPA degradation in the TNA-Cu PFC system involved better $\mathrm{e}^{-} / \mathrm{h}^{+}$separation and generation of other oxidants: in conventional photocatalytic process, hydroxyl radicals in liquid phase contributed to 93.7 \% MCPA degradation while only $2.4 \%$ MCPA degradation was mediated by other oxidants like $\cdot \mathrm{O}_{2}{ }^{-}, \mathrm{H}_{2} \mathrm{O}_{2}, \cdot \mathrm{HO}_{2}$; for MCPA degradation in the TNA-Cu PFC system, the contribution of hydroxyl radicals in the liquid phase decreased to $83.6 \%$, while contribution of other oxidants like $\cdot \mathrm{O}_{2}{ }^{-}, \mathrm{H}_{2} \mathrm{O}_{2}, \cdot \mathrm{HO}_{2}$ increased to $15.3 \%$. This change in MCPA degradation mechanisms was confirmed via degradation intermediates analysis by LC-MS/MS. The study on the effect of electrolyte concentration suggests that when operated under acidic conditions, addition of electrolyte is not required. The TNA-Cu PFC system was shown to work well in the presence of up to $15 \mathrm{mg} / \mathrm{L}$ natural organic matter (originating from two large rivers), high amounts of common inorganic ions, and even in WWTP effluent. The TNA-Cu PFC system also exhibited relatively good stability after several cycles of repeated use. The obtained results demonstrated that this is an adequate system for micropollutant removal from water at various places in the water cycle, i.e. as polisher of WWTP effluents before discharge or for cleaning intake water before producing drinking water. 


\subsection{Introduction}

In recent decades, emerging organic pollutants, including pharmaceutical compounds, herbicides, pesticides, and personal care products, are gaining increasing concerns because they are potentially hazardous to aquatic life and human health [18]. Among them, the synthetic carboxylic acid herbicide 4-chloro-2-methylphenoxyacetic acid (MCPA) has been detected in different water bodies [216, 217]. Toxicity and endocrine disrupting effects of MCPA on living species has been well studied, and abundantly evidenced in literature [218, 219]. However, owing to its poor biodegradability, conventional water treatment processes are usually insufficient for MCPA removal [224]. In addition, once the compound enters natural water bodies and becomes exposed to solar irradiation, more toxic compounds are generated from direct photolysis of MCPA [217].

Given this context, many water treatment techniques have been tested for cleaning MCPA contaminated water, and among them photocatalysis has received increasing research interests $[115,116]$, with a focus on $\mathrm{TiO}_{2}$. $\mathrm{TiO}_{2}$ is commonly used because it has relatively high quantum efficiency, easy accessibility, low toxicity, and high chemical/physical stability. $\mathrm{TiO}_{2}$ photocatalytic pollutants degradation processes involve production of electron/hole pairs $\left(\mathrm{e}^{-} / \mathrm{h}^{+}\right)$upon illumilation of $\mathrm{TiO}_{2}$ by radiant energy higher than its band gap energy (3.2 eV and $3.0 \mathrm{eV}$ for anatase and rutile respectively [104]), followed by formation of reactive oxygen species (ROS), e.g. hydroxyl radicals ( $\mathrm{OH})$, superoxide radical anions $\left(\cdot \mathrm{O}_{2}{ }^{-}\right)$, which has been extensively described in the literature [62, $103,110,111,113,114]$. A few attempts have been made to apply $\mathrm{TiO}_{2}$ for $\mathrm{MCPA}$ removal from water [116-118, 120, 225]. Most of the previous studies have been done in slurry systems with $\mathrm{TiO}_{2}$ nanoparticles suspensions. Although large surface area of $\mathrm{TiO}_{2}$ nanoparticles is beneficial for mass transfer, some major drawbacks are preventing the scale-up of such slurry systems: (1) costly liquid/solid separation process is required for retention and reuse of the catalyst particles; (2) light scattering by the catalyst particles; (3) the suspension of catalyst particles are unstable and easy to form aggregates in complex water matrices [144]. Therefore, immobilization of $\mathrm{TiO}_{2}$ should be considered. The electrochemical anodic produced $\mathrm{TiO}_{2}$ nanotube arrays (TNAs) is a promising alternative to conventional $\mathrm{TiO}_{2}$ suspensions. The TNAs exhibit multiple merits: (1) relatively large surface 


\section{Chapter 4}

area; (2) high chemical/mechanical stability; (3) oriented direction of electron transport which can reducing $\mathrm{e}^{-} / \mathrm{h}^{+}$pairs recombination; (4) relatively easy to make; (5) tunable morphologies. Examples of using of TNAs for organic pollutants elimination are available in literature [161-164, 210, 226, 227]. Up till now, very limited studies have been conducted to use TNAs for MCPA degradation [224].

Another major drawback of $\mathrm{TiO}_{2}$ photocatalytic (PC) processes is the fast recombination of $\mathrm{e}^{-} / \mathrm{h}^{+}$pairs, where a large amount of energy from absorbed photons is lost as heat, resulting in seriously reduced treatment efficiency [124]. Therefore, reducing the recombination of $\mathrm{e}^{-} / \mathrm{h}^{+}$pairs is a key, and efforts have been made on this aspect, e.g. integrating $\mathrm{TiO}_{2}$ with other active materials to form in-situ hetero-junction for better $\mathrm{e}^{-} / \mathrm{h}^{+}$ separation $[169,170]$, applying voltage bias - generally known as photoelectrocatalysis (PEC) - to separate $\mathrm{e}^{-/ h^{+}}$from the catalyst $[124,125]$. Recently a novel concept of PEC, known as photocatalytic fuel cell (PFC) has been introduced into the field of water treatment [171, 172, 228-231]. In PFC systems, the photo-induced electrons are able to migrate from $\mathrm{TiO}_{2}$ to the counter electrode through an external circuit driven by the photon-induced voltage between the two electrodes spontaneously. Hereby the photo-induced holes are left at the $\mathrm{TiO}_{2}$ surface triggering degradation of the target pollutants $[171,173]$. In this way, the recombination of $\mathrm{e}^{-/} / \mathrm{h}^{+}$pairs is reduced, and consequently enhanced target contaminants removal and simultaneous electricity production can be expected. The PFC concept is gaining increasing attention in the field of water treatment in recent years. Examples are available in literature reporting the use of PFC systems with various photoanodes and cathodes for treatment of different target pollutants, and enhanced degradation of organic pollutants compared with conventional photocatalysis [171, 173, 232]. However, very limited attempts have been made to apply PFC for micropollutant removal from water. In addition, little is known on micropollutants degradation mechanisms in PFC systems, effects of commonly present background water constituents, and applicability of PFC systems for micropollutant removal in real wastewater matrices.

In the present study we demonstrate enhanced MCPA degradation in a membranefree PFC system consisting of a TNA photoanode and a $\mathrm{Cu}$ foil acting as cathode (TNA-Cu PFC). Key influencing factors, i.e. initial $\mathrm{pH}$ and electrolyte concentration were investigated. 
In addition, to elucidate the mechanism of enhanced MCPA degradation in the TNA-Cu PFC system, contribution of different reactive oxygen species (ROS) were evaluated by adding specific scavengers and via analysis of degradation intermediates by LC-MS/MS. To test its applicability in a more complex water matrix, effects of natural organic matters (NOMs), commonly present inorganic ions, and real wastewater treatment plant (WWTP) effluent were studied. Furthermore, the stability of the system was evaluated over several reuse cycles.

\subsection{Materials and methods}

\subsubsection{Chemicals}

Ammonium sulphate ( $\geq 99.0 \%$ ), ammonium fluoride ( $\geq 98.0 \%)$, formic acid ( $\geq 96 \%)$, and tert-Butanol ( $\geq 99.0 \%$ ) were purchased from Sigma-Aldrich (Germany). Sulfuric acid (95\%), sodium hydroxide (1 mol/L aqueous solution), acetone ( $\geq 99.7 \%)$, sodium sulphate ( $\geq 99.0 \%$ ), and 2-propanol ( $\geq 99.9 \%$ ) were purchased from VWR (Belgium). MCPA ( $\geq 98.0 \%$ ) was purchased from Dr Ehrenstorfer GmbH (Germany). A stock solution of MCPA was prepared at concentration of $200 \mathrm{mg} / \mathrm{L}$. Dry solid extracts of two aquatic NOM Suwannee River NOM (2R101N) and Upper Mississippi River NOM (1R110N) were purchased from International Humic Substances Society (IHSS). $100 \mathrm{mg} / \mathrm{L}$ stock solutions of the two NOMs were prepared. Unless otherwise mentioned, all reaction solutions and stock solutions in this study were prepared with ultrapure water from a Milli-Q Advantage A10 system (Merck Millipore, Darmstadt, Germany).

\subsubsection{Preparation and characterization of TNA photoanode}

$\mathrm{TiO}_{2}$ nanotube arrays photoanode was prepared using a two-electrode electrochemical anodization cell connected to an EST150 DC power supply (Delta Elektronika, The Netherlands) as reported earlier by our group [210]. The anode was a $3 \mathrm{~cm}$ $\times 4.5 \mathrm{~cm}$ Titanium foil ( $\geq 99.5 \%, 0.3 \mathrm{~mm}$ thick), and the cathode was a $4.5 \mathrm{~cm} \times 5 \mathrm{~cm}$ Titanium plate (1 mm thick). The electrochemical anodization was performed in $100 \mathrm{~mL}$ aqueous electrolyte containing $0.15 \mathrm{M} \mathrm{NH} \mathrm{F}_{4}$ and $1 \mathrm{M}\left(\mathrm{NH}_{4}\right)_{2} \mathrm{SO}_{4}$ at $20 \mathrm{~V}$ for $2 \mathrm{~h}$, followed by thorough wash of the obtained TNAs and annealing at $600{ }^{\circ} \mathrm{C}$ for $0.5 \mathrm{~h}$. The surface morphologies of the TNAs electrode were characterized by scanning electron microscopy (SEM) and the 


\section{Chapter 4}

chemical composition of the prepared TNAs was measured by EDX. The SEM/EDX analyses were performed using a JEOL JSM 6480 scanning electron microscope (JEOL Europe B.V., Nieuw-Vennep, The Netherlands) equipped with a EDX system. Raman spectra of the prepared TNA were recorded using a Horiba LabRAM spectrometer equipped with a mpc3000 laser (532.2 nm), an $800 \mathrm{~mm}$ focal length achromatic flat field monochromator (grating of 600 grooves $/ \mathrm{mm}$ ) and a Synapse multichannel air cooled $\left(-70^{\circ} \mathrm{C}\right) \mathrm{CDD}$ detector.

\subsubsection{MCPA degradation experiments, apparatus and methods}

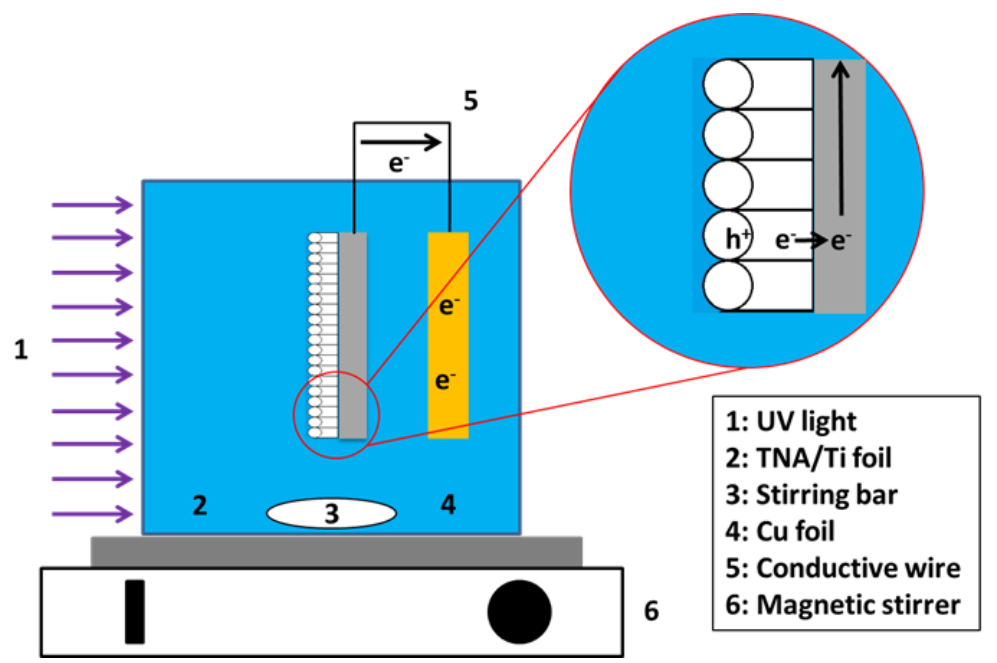

Figure 4. 1 Schematic of the experimental set-up

Figure 4.1 shows the schematic diagram of the experimental set-up of the TNA-Cu PFC system used for MCPA degradation. The experimental set-up was a two-electrode system, where the TNA electrode $(3 \mathrm{~cm} \times 3 \mathrm{~cm})$ was used as photoactive anode and a copper foil (Grade M2, $3 \mathrm{~cm} \times 4.5 \mathrm{~cm}$ ) was used as counter electrode and a rectangular quartz cube was used as reaction cell $(6 \mathrm{~cm} \times 6 \mathrm{~cm} \times 6 \mathrm{~cm})$. The distance between the two electrodes was set to be approx. $2 \mathrm{~cm}$. A UV-LED light source module was placed outside the reaction cell (on the side of TNA photoanode), which consisted of a $450 \mathrm{~mW}$ UV-LED emitting UV light with peak intensity at $365 \mathrm{~nm}$ (NCSU033B, NICHIA, Japan). The radiant power of the UV-LED that reached the surface of TNAs was $11.6 \mathrm{~mW} / \mathrm{cm}^{2}$, measured by a THORLABS S150C radiant power meter (THORLABS, USA). A reaction solution of $140 \mathrm{~mL}$ with $1.0 \mathrm{mg} / \mathrm{L}$ MCPA was used, containing, unless otherwise stated, $0.1 \mathrm{M} \mathrm{Na}_{2} \mathrm{SO}_{4}$ as supporting 
electrolyte and with unadjusted natural $\mathrm{pH}(\sim 6)$, unless specifically noted. For experiments using conventional photocatalysis method, only the copper foil cathode was removed from the system, while all other components of the set-up were kept unchanged. All MCPA degradation experiments were carried out in parallel duplicate, and average values of the duplicate were presented.

\subsubsection{Analytical procedures}

The output voltage and circuit current of the PFC system were measured by a Vertex potentiostat (Vertex One, Ivium Technologies B.V, The Netherlands) in the twoelectrode system. The open cell voltage $\left(V_{o c}\right)$ and short circuit current (Isc) were measured according to the method introduced in literature [232]. For MCPA concentrations and degradation by-products measurement, an Agilent LC-MS/MS system consisting of Agilent infinity 1260 LC-system (degasser, binary pump, auto sampler with cooled tray and column oven) and Agilent 6420 triple Quadrupole Mass Spectrometer with Electrospray ion source was used. More detailed information of the analytical method used can be found in our previous study [65].

\subsection{Results and discussion}

\subsubsection{Characterization of $\mathrm{TiO}_{2}$ nanotube arrays photoanode}

Surface morphology of the TNA photoanode is shown in Figure 4. 2a. It can be seen that the TNA anode is covered by a self-organised tubular structure layer, which has an average pore size of around $80 \mathrm{~nm}$. It has been reported that such a nanotubular structure results in oriented electron transport from $\mathrm{TiO}_{2}$ surface to Ti which can facilitate e-/h+ pairs ' separation in photoelectrocatalytic processes [233]. The Raman spectrum of the TNA anode surface shown in Figure 4. $2 \mathrm{~b}$ demonstrates that the TNA layer has a crystallographic phase consisting of both anatase and rutile. Good photocatalytic performance of $\mathrm{TiO}_{2}$ contains both anatase and rutile has been documented in literature [203, 210]. 

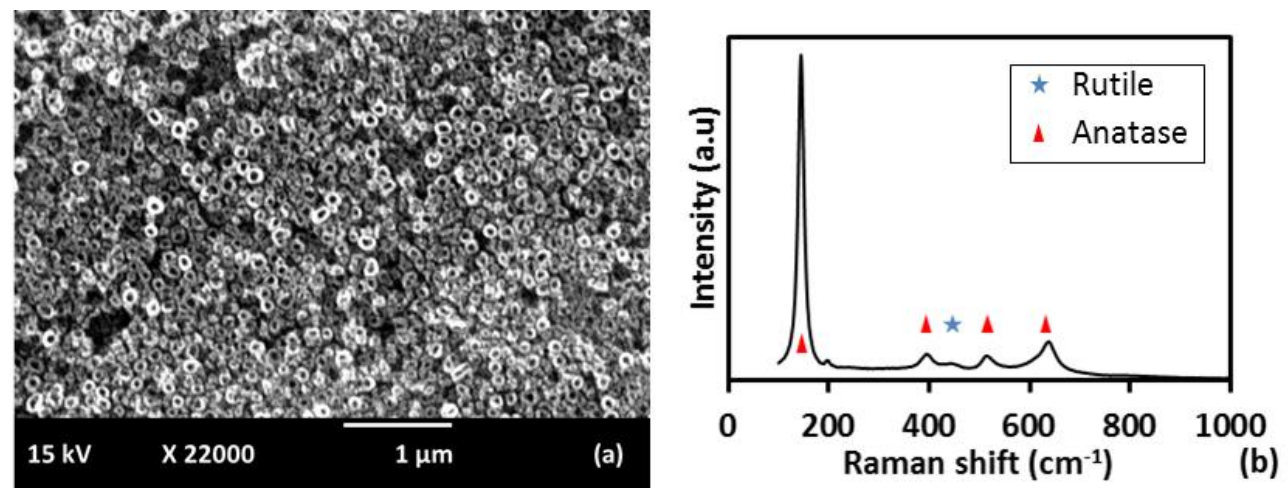

Figure 4. 2 SEM top view image (a) and Raman spectrum (b) of the TNA photoanode surface

\subsubsection{MCPA removal in the TNA-Cu PFC system}

\subsubsection{Enhanced MCPA removal in the TNA-Cu PFC system}

The efficacy of different methods, i.e. photocatalysis (PC), photocatalytic fuel cell (PFC), on MCPA removal was evaluated, and the results are shown in Figure 4. 3. Compared with TNA based photocatalysis, the MCPA removal by the PFC method was successfully enhanced: after 120 min of treatment, the PC method resulted in 52.2\% MCPA removal and the PFC method resulted in 62.0\% MCPA removal. The initial apparent rate constant $\left(k_{a p p}\right)$ for MCPA degradation was found $0.0058 \mathrm{~min}^{-1}$ for the PC system, and $0.0072 \mathrm{~min}^{-1}$ for the TNA-Cu PFC system. The obtained results suggest that MCPA degradation can be effectively enhanced by using the TNA-Cu PFC system. Successful enhancement of various organic pollutants degradation over photocatalysts by adding a cathode material has been reported in recent years. Li et al. (2014)[173] applied $\mathrm{TiO}_{2}$ (coated on Ti substrate) as photocatalyst and a platinized $\mathrm{Ti}$ as cathode to degrade Reactive Brilliant Red $\mathrm{X}-3 \mathrm{~B}$, a dye, from wastewater. They found that the colour removal after $80 \mathrm{~min}$ of treatment could reach $85 \%$ by the PFC method, while the conventional PC method without cathode connection could achieve only $55 \%$ colour removal under the same conditions. Such enhancement was due to better $\mathrm{e}^{-} / \mathrm{h}^{+}$separation achieved by electron transfer from catalyst to cathode assisted by the self-bias between the two electrodes, according to the authors [173]. The same phenomenon was reported by Du et al. (2014): enhanced photocatalytic methyl orange degradation was obtained when a Pt/C coated bio-cathode was applied, because better 
$\mathrm{e}^{-} / \mathrm{h}^{+}$separation was achieved by the bias between the $\mathrm{TiO}_{2}$ catalyst electrode and the biocathode.

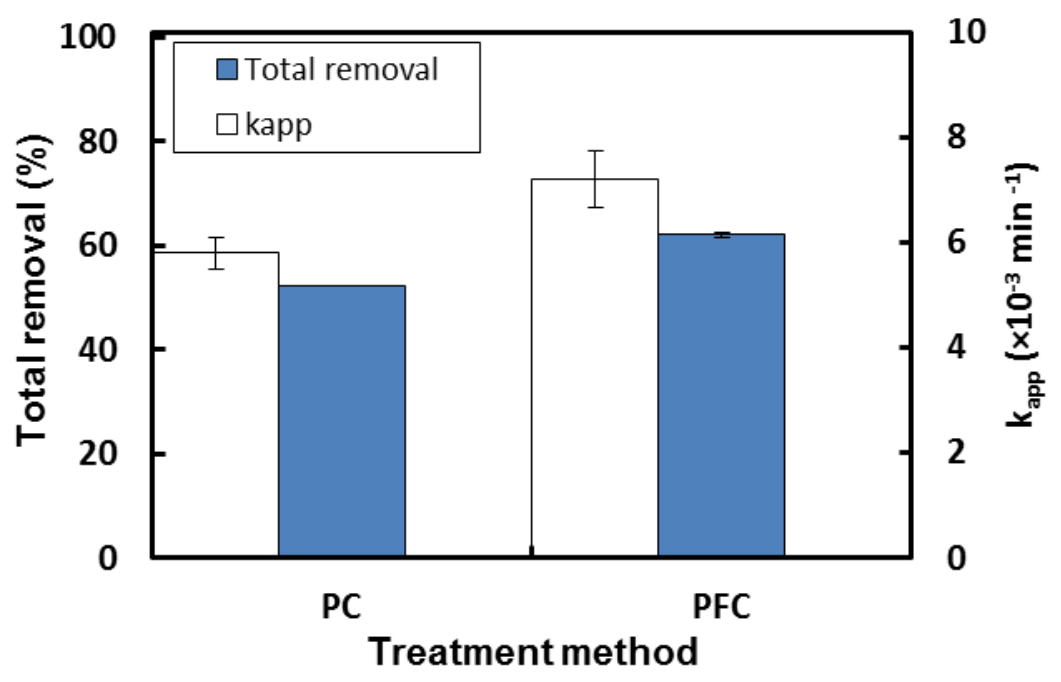

Figure 4. 3 Total removal (after $\mathbf{1 2 0}$ min treatment) and initial apparent degradation rate constant of MCPA by PC method and TNA-Cu PFC method. Conditions: $[M C P A]_{0}=1.0$ $\mathrm{mg} / \mathrm{L}$, natural pH ( 6), $0.1 \mathrm{M} \mathrm{Na}_{2} \mathrm{SO}_{4}$ as electrolyte.
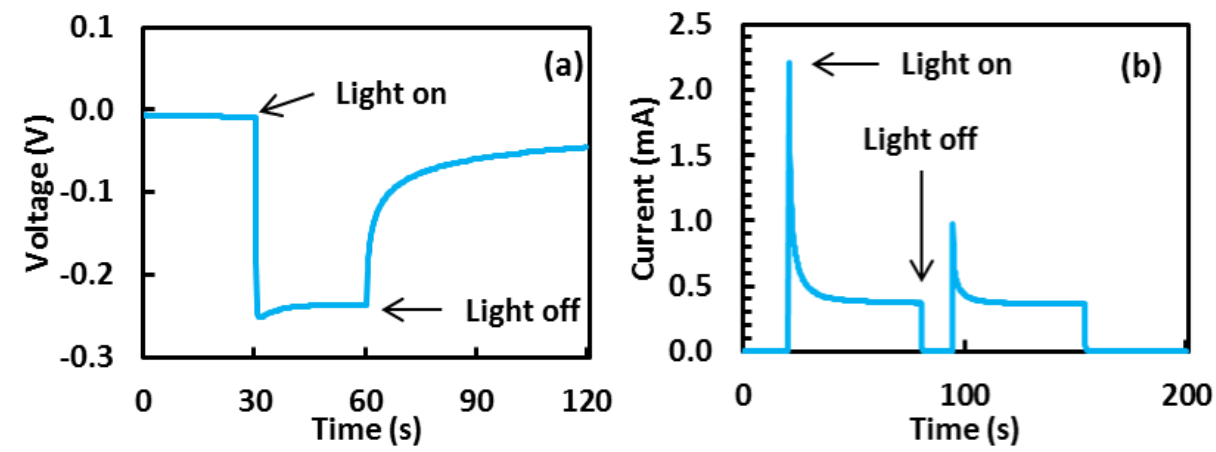

Figure 4. 4 Open cell voltage (Voc) (a) and short circuit current (Isc) (b) of the TNA-Cu PFC system. Conditions: $[\mathrm{MCPA}]_{0}=1.0 \mathrm{mg} / \mathrm{L}$, natural pH ( 6), $0.1 \mathrm{M} \mathrm{Na}_{2} \mathrm{SO}_{4}$ as electrolyte.

Figure 4. 4a shows that a $V_{o c}$ value of $0.24 \mathrm{~V}$ was generated in the TNA-CU PFC system upon UV illumination. The $V_{o c}$ is a measure of accumulation of photo-generated electrons in the TNA anode upon illumination, as previously explained by Chen et al., [234]. 


\section{Chapter 4}

As shown in Figure 4. 4b, upon UV irradiation, the TNA-Cu PFC system generated rapid photocurrent response, with a steady state value of about $0.37 \mathrm{~mA}$, which represent the photo-generated electrons migration from the TNA anode to the $\mathrm{Cu}$ cathode.

Experiments in tap water samples were conducted to evaluate the effect of natural water constituents (Table S4. 1 shows the characteristics of the tap water samples). In tap water samples, although MCPA degradation performances of both the conventional PC system and the TNA-Cu PFC system were reduced, the TNA-Cu PFC system still resulted in much more rapid MCPA degradation compared with that in the conventional PC system, as shown in Figure 4. 5. The open cell voltage of the TNA-Cu PFC system operated in tap water was measured to be nearly $0.35 \mathrm{~V}$, and the short circuit current was nearly $0.3 \mathrm{~mA}$ (Figure S4. 1).

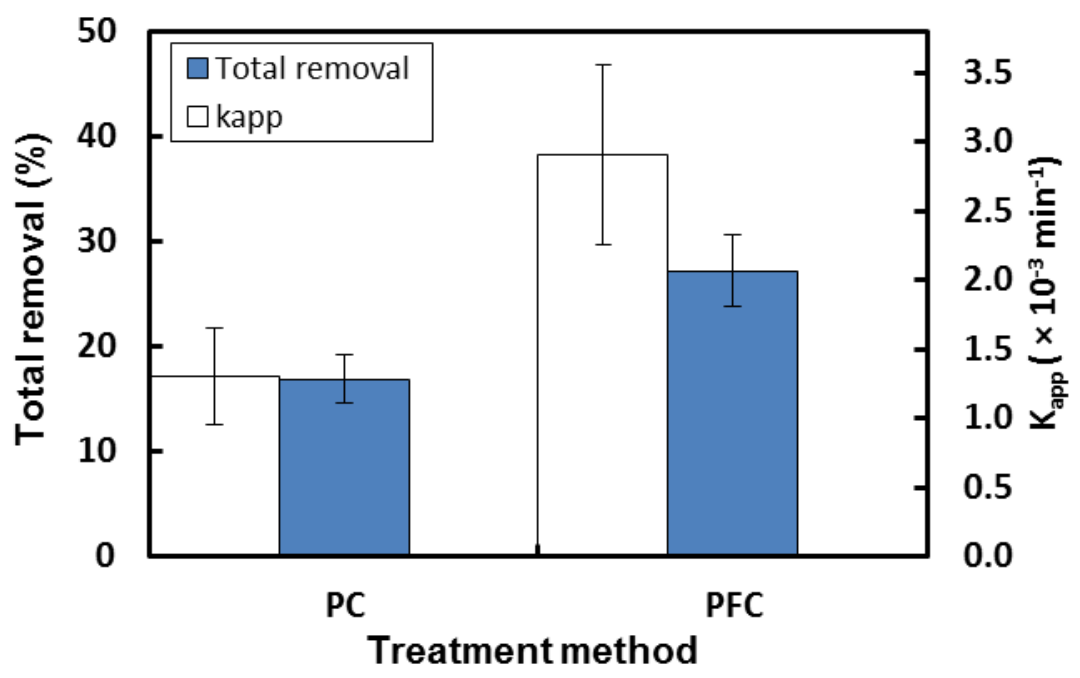

Figure 4. 5 Total removal (after $120 \mathrm{~min}$ treatment) and initial apparent degradation rate constant of MCPA in tap water samples by PC method and PFC method. Conditions:

$[\mathrm{MCPA}]_{0}=1.0 \mathrm{mg} / \mathrm{L}$, natural pH ( 6), no electrolyte addition.

The results presented herein demonstrate that, compared with the PC system, applying the TNA-Cu PFC system is a facile and effective way to enhance the removal of MCPA (and potentially also other micropollutants), and can simultaneously produce electricity for other low-power requiring applications. 


\subsubsection{Crucial role of acidic $\mathrm{pH}$ to promote MCPA degradation efficiency}

It has been documented that the $\mathrm{pH}$ of the reaction solution can affect the performance of various photochemical systems on degradation of organic pollutants [106, 235]. Therefore, degradation of MCPA was studied at different initial $\mathrm{pH}\left(\mathrm{pH}_{0}\right)$ levels (ranging from 3 to 11 ) to examine the effect of $\mathrm{pH}$ on the performance of the TNA-Cu PFC system. Control experiments were performed under the same conditions in the PC system. The solution $\mathrm{pH}$ was nearly constant during treatment, so the effect of $\mathrm{pH}$ fluctuation can be neglected.

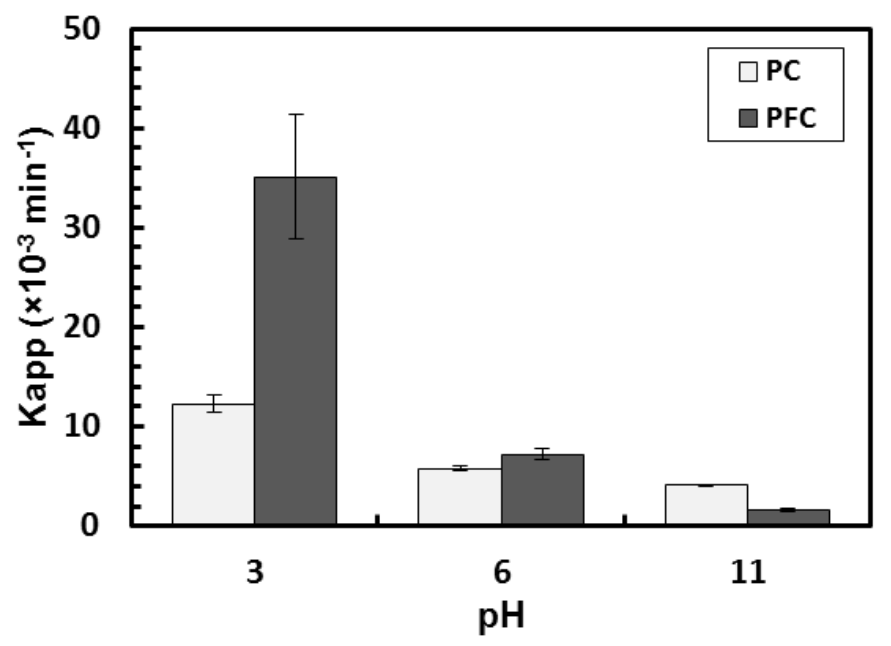

Figure 4. 6 Effect of $\mathrm{pH}$ on the initial apparent MCPA degradation rate constant $\left(\mathrm{k}_{\mathrm{app}}\right)$ by PC method and PFC method. Conditions: $[\mathrm{MCPA}]_{0}=1.0 \mathrm{mg} / \mathrm{L}, \mathrm{pH}_{0}$ adjusted by $\mathrm{H}_{2} \mathrm{SO}_{4}$ or $\mathrm{NaOH}$ solutions, $0.1 \mathrm{M} \mathrm{Na}_{2} \mathrm{SO}_{4}$ as electrolyte.

As shown in Figure 4. 6, better performance was achieved with lower $\mathrm{pH}$ level in both the PC system and the TNA-Cu PFC system. The obtained results (Figure 4. 6) show that acidic $\mathrm{pH}\left(\mathrm{pH}_{0}=3\right)$ plays a significant role in enabling effective degradation: (1) the fastest MCPA degradation in the PC system and the TNA-Cu PFC system were both achieved at acidic $\mathrm{pH}\left(\mathrm{pH}_{0}=3\right)$; (2) under same conditions at acidic $\mathrm{pH}, \mathrm{MCPA}$ degradation in the TNACu PFC system (with initial kapp of $0.0351 \mathrm{~min}^{-1}$ ) was almost three times faster than that in the PC system (with initial kapp of $0.0123 \mathrm{~min}^{-1}$ ). This demonstrates that acidic conditions are strongly favourable for the pollutant degradation by the TNA-Cu PFC system. This 


\section{Chapter 4}

finding is in line with previous studies with other pollutants. In a study on a dual photoelectrodes ferrous ions PFC system conducted by Zhao et al. [236], it was reported that lower $\mathrm{pH}$ would be beneficial for the $\mathrm{PFC}\left(\mathrm{Fe}^{2+}\right)$ system pollutant degradation performance, the same effect of low pH on a conventional PFC system was also reported. In another study conducted by the same authors Zhao et al. [232] on a Fenton-PFC system, the same findings were presented, and this phenomenon was attributed to two facts: (1) other oxidants, e.g. $\cdot \mathrm{O}_{2}{ }^{-}, \mathrm{H}_{2} \mathrm{O}_{2}, \cdot \mathrm{HO}_{2}$, can be more easily generated at the cathode under acidic conditions; (2)a positively charged TNA anode surface would be beneficial to degradation of organic pollutants on the catalyst surface. Liu et al. [237] studied diclofenac photoelectrocatalytic degradation in a persulfate assisted PEC (external voltage was supplied to drive electron transmission) system, and reported that diclofenac degradation decreased with an increase of $\mathrm{pH}_{0}$. This finding was explained by higher redox potential $\cdot \mathrm{SO}_{4}{ }^{-}$(derived from the $\mathrm{Na}_{2} \mathrm{SO}_{4}$ electrolyte) at acidic $\mathrm{pH}$ in their studied system. These explanations are also reasonable to our findings that acidic $\mathrm{pH}$ is favourable to MCPA degradation in both the PC system and the TNA-Cu PFC system. Moreover, in the present study, beneficial effect of acidic $\mathrm{pH}$ was even more profound in the TNA-Cu PFC system. Considering that the same $\mathrm{Na}_{2} \mathrm{SO}_{4}$ concentration and the same TNA was applied as photocatalyst in the PC system and the PFC system, the promotion of pollutant degradation on the TNA surface, and redox potential of $\cdot \mathrm{SO}_{4}^{-}$at same acidic conditions should be the same for both systems. Therefore, in the TNA-Cu PFC system, the increased generation of other oxidants is likely the crucial factor that contributes to the enhanced MCPA degradation performance under acidic conditions. Since the best MCPA degradation performance was achieved under acidic condition, the remaining experiments were performed with a $\mathrm{pH}_{0}$ value of 3 .

\subsubsection{Degradation mechanisms}

The working principle of PFC systems has been introduced in literature [173, 232]. Figure 4.7 is a schematic illustration of the TNA-Cu PFC working principle. Upon excitation by photons with energy larger than $\mathrm{TiO}_{2}$ band gap, electrons $\left(\mathrm{e}^{-}\right)$will migrate from the valence band to conduction band and leave positively charged holes $\left(\mathrm{h}^{+}\right)$in the valence band of the $\mathrm{TiO}_{2}$ material. The $\mathrm{h}^{+}$is a very powerful oxidant able to degrade organic pollutants adsorbed on the $\mathrm{TiO}_{2}$ surface. The $\mathrm{h}^{+}$can also react with water molecules $\mathrm{or}^{-} \mathrm{OH}^{-}$to generate 
hydroxyl radical $(\cdot \mathrm{OH})$, which is a well-known strong oxidant that rapidly degrade broad range of organics in various advanced oxidation processes. Part of the $\cdot \mathrm{OH}$ is adsorbed on the TNA surface $(\cdot \mathrm{OH}$ surface) and can readily degrade organic pollutants that diffused towards and adsorbed onto the TNA surface. The rest of the $\cdot \mathrm{OH}$ radicals can diffuse into the liquid phase $(\cdot \mathrm{OH}$ free) and react with organic pollutants there. Usually organic pollutants are degraded predominantly by $\mathrm{h}^{+}$and $\cdot \mathrm{OH}$ in photocatalytic processes $[148,210]$. The conduction band electrons can be quenched rigorously by various electron accepters in the reaction solution to generate water and/or other oxidant like $\cdot \mathrm{O}_{2}^{-}, \mathrm{H}_{2} \mathrm{O}_{2}, \cdot \mathrm{HO}_{2}$. In conventional photocatalytic systems, the conduction electrons can easily migrate back to valence band and recombine with the holes. This recombination of $\mathrm{h}^{+} / \mathrm{e}^{-}$pairs is a limiting factor of photocatalytic processes, because such recombination leads to energy loses as heat and less availability of $\mathrm{h}^{+}$for subsequent $\cdot \mathrm{OH}$ production and organic pollutant degradation. In a PFC system, by applying an external circuit and a cathode, the photo generated electrons will be more separated from the valence band holes via transferring to the cathode material through the external circuit. In this way, recombination of $\mathrm{h}^{+} / \mathrm{e}^{-}$pairs can be largely suppressed and more valence band holes are available for $\cdot \mathrm{OH}$ production and organic pollutant degradation, and more electrons are available for generation of other oxidant like $\cdot \mathrm{O}_{2}^{-}, \mathrm{H}_{2} \mathrm{O}_{2}, \cdot \mathrm{HO}_{2}$. Thus, enhanced organic pollutant degradation and electricity generation are achieved in the PFC system simultaneously.

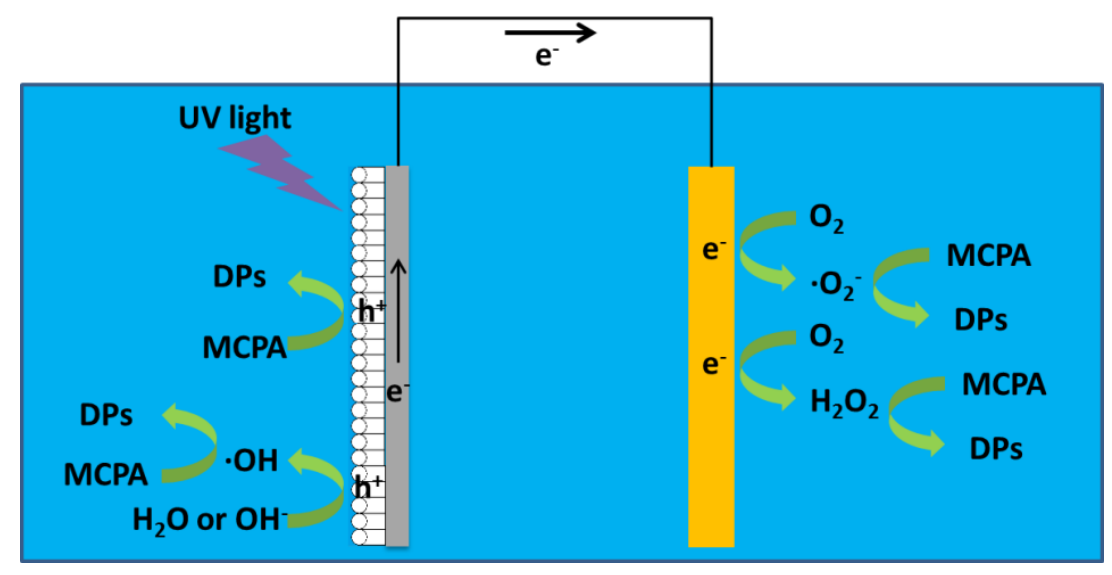

Figure 4. 7 Schematic of proposed degradation mechanism of MCPA in the PFC system 


\section{Chapter 4}

To elucidate contribution of the above mentioned degradation routes, experiments with addition of specific oxidant scavengers, i.e. formic acid (FA) and tertbutanol (TBA), were carried out in both the PC system and the TNA-Cu PFC system. Because formic acid can rigorously quench photo generated electron holes $\left(\mathrm{h}^{+}\right)$on the surface of TNA once they were produced, addition of formic acid in the reaction hindered not only the degradation pathway mediated by electron holes but also degradation pathways with participation of hydroxyl radicals [104]. Due to extreme low affinity to $\mathrm{TiO}_{2}$ surfaces, the tert-butanol only scavenges hydroxyl radicals in bulk liquid phase. The results (Figure 4. 8) show that the MCPA degradation in the PC system was decreased from $81.5 \%$ to $5.1 \%$ and 1.9 \% by adding tert-butanol and formic acid, respectively; while the MCPA degradation in the TNA-Cu system was decreased from $99.4 \%$ to $16.3 \%$ and $15.2 \%$ by adding tert-butanol and formic acid, respectively. 

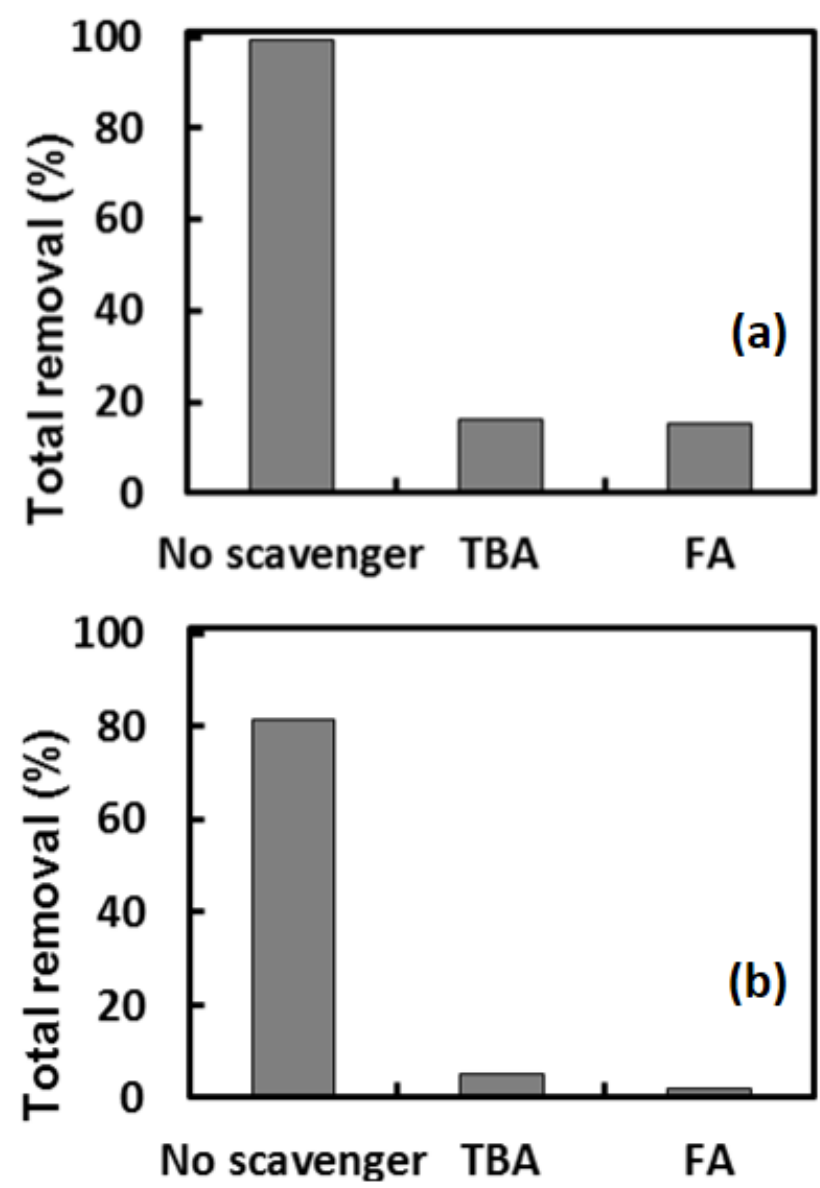

Figure 4. 8 Effect of different scavengers on the total removal of MCPA after $120 \mathrm{~min}$ treatment by PFC method (a) and PC method (b). Conditions: $[\mathrm{MCPA}]_{0}=1.0 \mathrm{mg} / \mathrm{L}, \mathrm{pH}_{0}=3$, $\left[\mathrm{Na}_{2} \mathrm{SO}_{4}\right]=0.1 \mathrm{M}$.

From the obtained results, the contribution of different reaction routes was understood. Figure 4. 9 illustrates the contribution of different oxidants to MCPA degradation in the PC system and the TNA-Cu PFC system. In the conventional PC system, reactions with $\cdot \mathrm{OH}$ free was the predominant MCPA degradation route (accounts for $93.7 \%$ MCPA degradation), while a very small portion of MCPA was degraded by $\mathrm{h}^{+}$and $\cdot \mathrm{OH}$ surface (accounts for $3.9 \%$ MCPA degradation), and other oxidant species, e.g. $\cdot \mathrm{O}_{2}{ }^{-}, \mathrm{H}_{2} \mathrm{O}_{2}, \cdot \mathrm{HO}_{2}$ (accounts for $2.4 \%$ MCPA degradation). However, in the TNA-Cu PFC system, although 


\section{Chapter 4}

primary MCPA degradation oxidant was still $\cdot \mathrm{OH}_{\text {free }}$ (accounts for $83.6 \% \mathrm{MCPA}$ degradation), the contribution of other oxidants (e.g. $\cdot \mathrm{O}_{2}{ }^{-}, \mathrm{H}_{2} \mathrm{O}_{2}, \cdot \mathrm{HO}_{2}$ ) became a significant part (accounts for 15.3 \% MCPA degradation). The same findings were also found under neutral pH (shown in Figure S4. 3). The increased contribution of other oxidants, e.g. $\cdot \mathrm{O}_{2}{ }^{-}, \mathrm{H}_{2} \mathrm{O}_{2}, \cdot \mathrm{HO}_{2}$, in the TNA-CU PFC system can be ascribed to reduced $\mathrm{e}^{-} / \mathrm{h}^{+}$recombination. In the TNA-Cu system, photo-generated electrons were transferred to the $\mathrm{Cu}$ cathode via external circuit by the system self-bias and therefore better separation of $\mathrm{h}^{+} / \mathrm{e}^{-}$pairs were achieved. Consequently, on the one hand more $\mathrm{h}^{+}$were available to trigger $\mathrm{h}^{+}$and $\cdot \mathrm{OH}$ mediated MCPA degradation, on the other hand, more $\mathrm{e}^{-}$were available to produce other oxidants like $\cdot \mathrm{O}_{2}^{-}, \mathrm{H}_{2} \mathrm{O}_{2}, \cdot \mathrm{HO}_{2}$ (shown in equations 1-3 below) and subsequent MCPA degradation. Such enhancement of micropollutant removal by better $\mathrm{h}^{+} / \mathrm{e}^{-}$separation and generation of oxidants like $\cdot \mathrm{O}_{2}^{-}$, $\mathrm{H}_{2} \mathrm{O}_{2}, \cdot \mathrm{HO}_{2}$ is in accordance with literature [232]. In a study performed by Zhao et al. (2017) on enhanced organic pollutant removal in a Fenton-PFC system, the involvement of $\cdot \mathrm{O}_{2}^{-}$, $\mathrm{H}_{2} \mathrm{O}_{2}, \cdot \mathrm{HO}_{2}$ was also proposed.

$$
\begin{aligned}
& \mathbf{O}_{2}+\mathbf{e}^{-} \rightarrow \cdot \mathrm{O}_{2}^{-} \\
& \mathbf{0}_{2}+2 \mathbf{H}^{+}+2 \mathbf{e}^{-} \rightarrow \mathbf{H}_{2} \mathbf{O}_{2} \\
& \cdot \mathrm{O}_{2}^{-}+\mathbf{e}^{-} \rightarrow \cdot \mathrm{HO}_{2}
\end{aligned}
$$

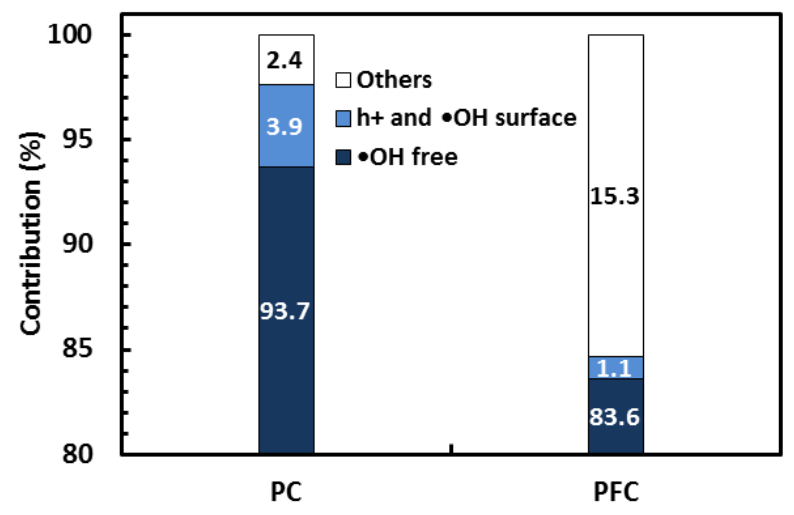

Figure 4. 9 Contribution of different oxidants on MCPA degradation by PC method and PFC method. Conditions: $[\mathrm{MCPA}]_{0}=1.0 \mathrm{mg} / \mathrm{L}, \mathrm{pH}_{0}=3,\left[\mathrm{Na}_{2} \mathrm{SO}_{4}\right]=0.1 \mathrm{M}$. 

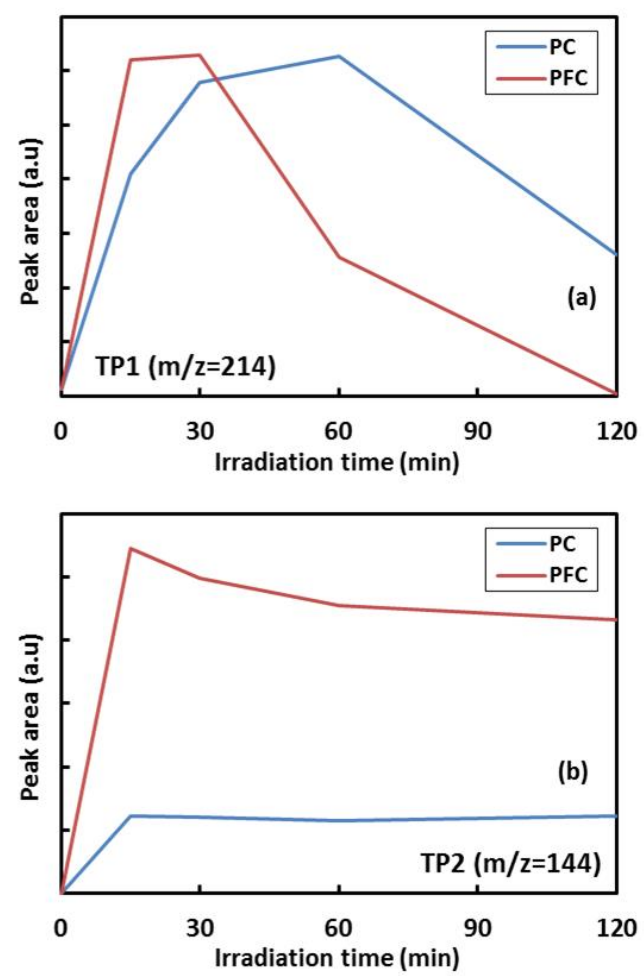

Figure 4. 10 MCPA degradation transformation products formation during PC treatment and PFC treatment. Conditions: $[\mathrm{MCPA}]_{0}=1.0 \mathrm{mg} / \mathrm{L}, \mathrm{pH}_{0}=3,\left[\mathrm{Na}_{2} \mathrm{SO}_{4}\right]=0.1 \mathrm{M}$.

The difference in MCPA degradation in PC and PFC systems was further confirmed via transformation products analysis by LC-MS/MS. Two main transformation products (TP) were identified and their tentative structures were proposed based on literature: one with a molecular weight of 214 and retention time of $2.4 \mathrm{~min}$ (TP1) which is likely to be resulted from addition of hydroxyl group to the MCPA molecule by hydroxyl radical attack [120], and the other with a molecular weight of 144 and retention time of $0.84 \mathrm{~min}$ (TP2) [120]. The results show that evolution of two main transformation products during different treatment varied (Figure 4. 10): TP1 formation and degradation in the PFC system were much more rapid than that in the PC system, and much more TP2 was formed in the PFC system than in the PC system. 


\subsubsection{Effect of operational parameters and water constituents on MCPA removal}

\subsubsection{Effect of electrolyte concentration}

Electrolyte concentration is known as an important operational parameter in photocatalytic systems and photoelectrocatalytic systems (including photocatalytic fuel cell systems) $[232,238]$. MCPA experiments were conducted in both conventional PC system and the TNA-Cu system with different $\mathrm{Na}_{2} \mathrm{SO}_{4}$ concentrations, namely $0 \mathrm{M}, 0.01 \mathrm{M}, 0.1 \mathrm{M}$, and $0.5 \mathrm{M}$ (resulted in conductivity ranging from 0.48 to $59.48 \mathrm{mS} / \mathrm{cm}$ ), at acidic conditions $\left(\mathrm{pH}_{0}=3\right)$.

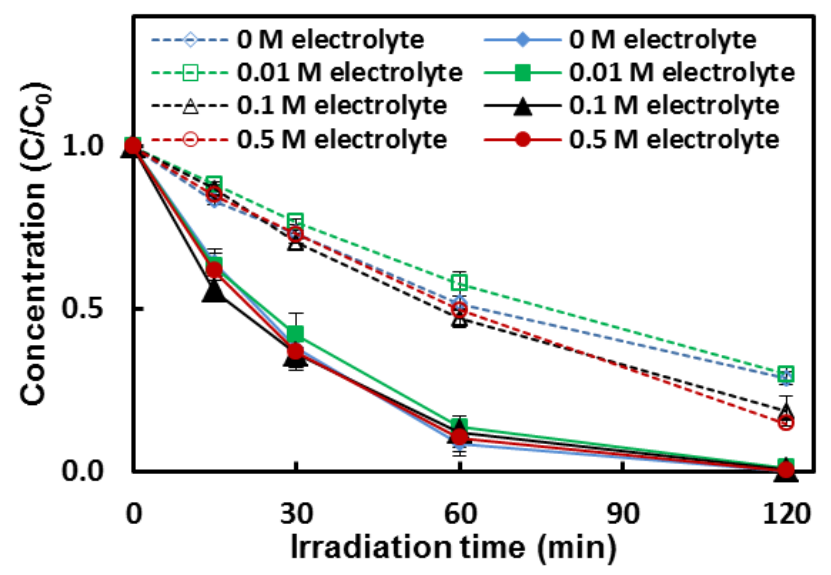

Figure 4. 11 Effect of electrolyte concentration on the MCPA removal by PC method (dashed line) and PFC method (solid line). Conditions: $[\mathrm{MCPA}]_{0}=1.0 \mathrm{mg} / \mathrm{L}, \mathrm{pH}_{0}=3$.

The results (Figure 4. 11) show that electrolyte concentration in the tested range imposes no significant impact on the MCPA degradation performance of both systems. Even without addition of $\mathrm{Na}_{2} \mathrm{SO}_{4}$, MCPA degradation in the TNA-Cu PFC system was still greatly enhanced compared with that in the PC system: degradation of MCPA in the TNA-Cu PFC system $\left(k_{a p p}=0.0389 \mathrm{~min}^{-1}\right)$ was nearly 3.6 times faster than that in the PC system $\left(k_{a p p}=\right.$ $0.0109 \mathrm{~min}^{-1}$ ). This reveals that, under acidic conditions, the reaction solution conductivity is not a limiting factor on the separation of $\mathrm{h}^{+} / \mathrm{e}^{-}$pairs, and consequently oxidants production and MCPA degradation were not affected by varied electrolyte concentration. Therefore, unless otherwise stated, remaining experiments were conducted without $\mathrm{Na}_{2} \mathrm{SO}_{4}$ addition. 


\subsubsection{Effect of background organic matters}

Various types of natural organic matter (NOMs) are commonly present in various water bodies and are known to be able to interfere with many AOPs [177]. Given this context, to evaluate the applicability of the TNA-Cu PFC system in real life application, it is of interest to study the effect of NOMs and document the allowable NOMs concentrations range. Therefore, experiments were carried out in the presence of different reference NOMs at varied concentrations (i.e. $5 \mathrm{mg} / \mathrm{L}, 15 \mathrm{mg} / \mathrm{L}$ ).

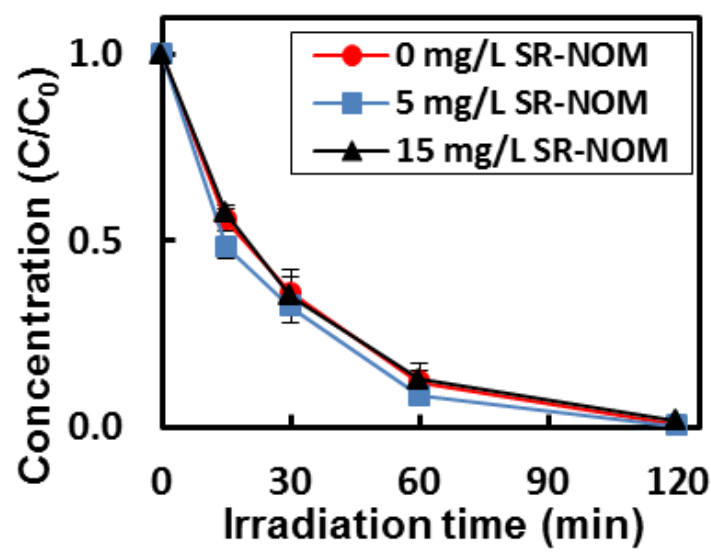

(a)

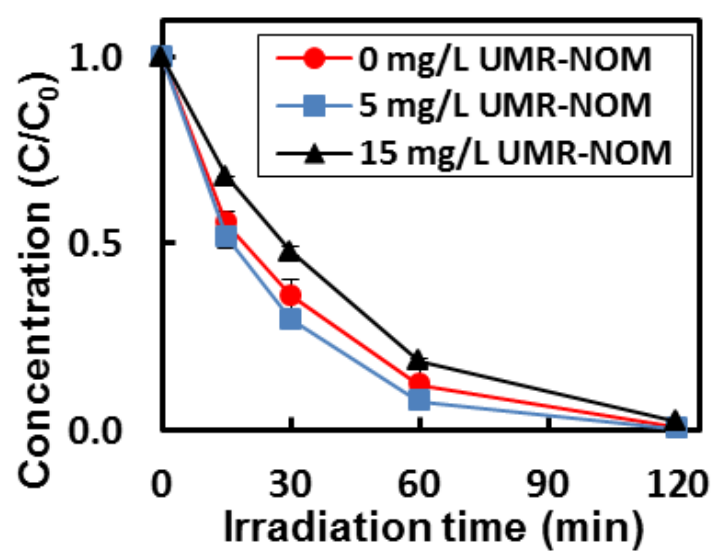

(b)

Figure 4. 12 Effect of different natural organic matters on the MCPA removal in the TNACu PFC system: (a) Suwannee River NOM; (b) Upper Mississippi River NOM. Conditions: $[\mathrm{MCPA}]_{0}=1.0 \mathrm{mg} / \mathrm{L}, \mathrm{pH}_{0}=3$, no addition of electrolyte.

The results (Figure 4. 12) indicate that the presence of NOMs has no significant detrimental effect on MCPA degradation performance of the TNA-Cu PFC system: the 


\section{Chapter 4}

presence of up to $15 \mathrm{mg} / \mathrm{L}$ Suwannee River NOM (SR-NOM) had no noteworthy effect on MCPA degradation (Figure 4. 12a); while the presence of up to $15 \mathrm{mg} / \mathrm{L}$ Upper Mississippi River NOM (UMR-NOM) only caused a slight decrease in the MCPA degradation rate (Figure 4. 12b). NOMs usually interfere with photocatalytic system by blocking the active site of the catalyst surface, and competing for reactive oxidant species against target pollutants [142, 143]. Therefore, the null effect of NOMs on the TNA-Cu system could be attributed to two facts: (1) under acidic condition, adsorption of NOMs onto the TNA surface and the subsequent blocking of the active site is greatly suppressed; (2) in the TNA-Cu system, contribution of more selective oxidants like $\cdot \mathrm{O}_{2}{ }^{-}$can compensate the $\cdot \mathrm{OH}$ scavenging effect of NOMs.

\subsubsection{Effect of inorganic ions}

Besides organic matter, many commonly present inorganic species, e.g. nitrate ions, chloride ions, calcium ions, are also known to be able to interfere with $\mathrm{TiO}_{2}$ based photocatalytic processes $[106,166,239]$. Therefore, experiments were conducted in presence of various concentration levels of these inorganic ions to evaluate their impact on MCPA degradation performance of the TNA-Cu PFC system.

The results (Figure 4. 13) suggest that both nitrate ions and calcium ions had no detrimental effect on the MCPA degradation performance of the TNA-Cu PFC system. As reported in previous studies, chloride ions can strongly adsorb onto $\mathrm{TiO}_{2}$ surface and block the active site under acidic conditions [239]. Interestingly, in contrast to conventional photocatalytic systems (example can be seen in Figure S4. 4) the presence of chloride ions could even significantly promote MCPA degradation in the TNA-Cu PFC system. This finding could be attributed to the fact that chlorine based strong oxidants (e.g. $\mathrm{ClO}^{-}$) could be produced in the TNA-Cu PFC system, and stimulates MCPA degradation. 


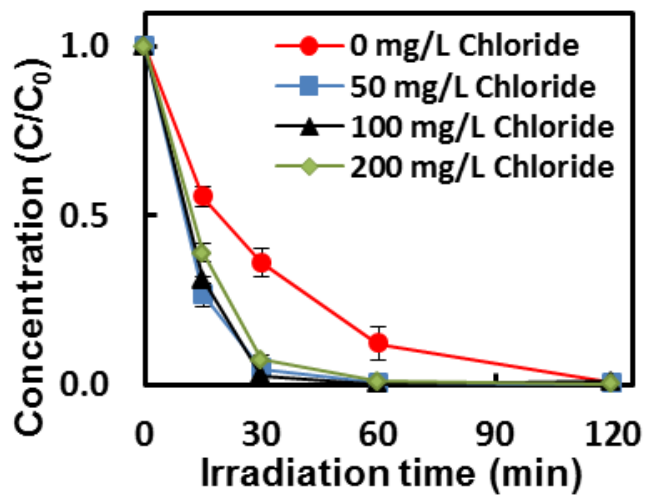

(a)

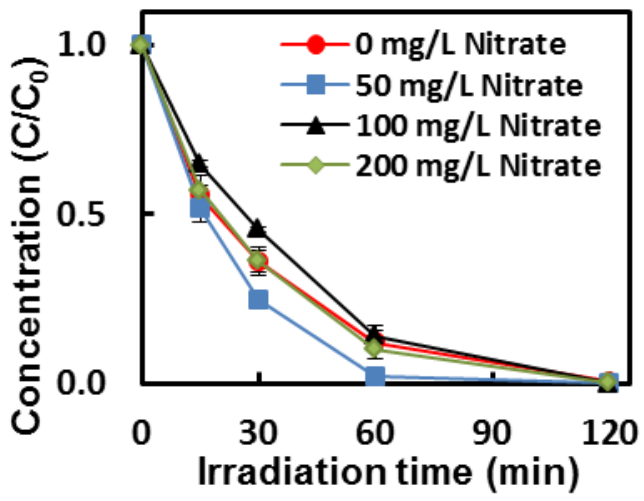

(b)

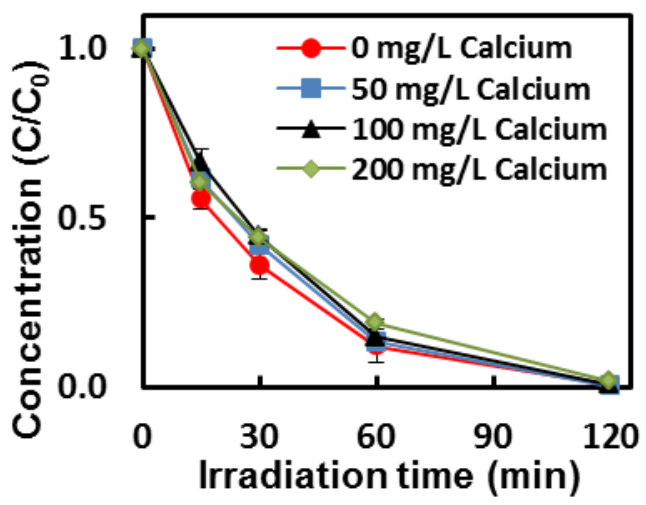

(c)

Figure 4. 13 Effect of common inorganic ions on the MCPA removal in the TNA-Cu PFC system: (a) Chloride ions; (b) Nitrate ions; (c) Calcium ions. Conditions: $[\mathrm{MCPA}]_{0}=1.0$

$$
\mathrm{mg} / \mathrm{L}, \mathrm{pH}_{0}=3 \text {. }
$$

In a study conducted by Lee, et al. employing a $\mathrm{ZnO} / \mathrm{Zn}$ based PFC system for removal of an azo dye Reactive Green 19 (RG 19), it is reported that the presence of chloride 


\section{Chapter 4}

ions could promote the oxidation of RG 19 . The same finding was very recently reported by another research group employing graphite-PVC composite based electrochemical oxidation system for diclofenac degradation [240]. The formation of hypochlorite ions was described in the following equations:

$$
\begin{aligned}
& 2 \mathrm{Cl}^{-}-\mathrm{e}^{-} \rightarrow \mathrm{Cl}_{2} \\
& \mathrm{Cl}_{2}+\mathrm{H}_{2} \mathrm{O} \rightarrow \mathrm{HOCl}+\mathrm{H}^{+}+\mathrm{Cl}^{-} \\
& \mathrm{HOCl} \rightarrow \mathrm{H}^{+}+\mathrm{OCl}^{-}
\end{aligned}
$$

The results presented herein indicate that the TNA-Cu PFC system has the potential to overcome interference of common inorganic ions in complex water matrices.

\subsubsection{MCPA removal and simultaneous electricity generation in real wastewater treatment plant effluent}

In order to better evaluate applicability of TNA-Cu PFC system in real wastewater treatment implications, MCPA removal experiments were also done in wastewater treatment plant effluent (WWTPE) sample. The WWTPE was collected from the secondary biological treatment facility (activated sludge method) of a municipal wastewater treatment plant located in Sneek (Friesland, The Netherlands), characteristics of the WWTPE sample were shown in Table 4. 1. MCPA was spiked into the WWTPE sample to an initial concentration of $1.0 \mathrm{mg} / \mathrm{L}$ and the initial $\mathrm{pH}$ of reaction solution was adjusted to 3 by adding

\begin{tabular}{|c|c|c|c|}
\hline Parameters & Concentration (mg/L) & Parameters & Concentration $(\mathrm{mg} / \mathrm{L})$ \\
\hline COD & 39.1 & IC & 55 \\
\hline $\mathrm{PO}_{4}{ }^{3-}-\mathrm{P}$ & 0.68 & $\mathrm{Ca}^{2+}$ & $>20$ \\
\hline $\mathrm{SO}_{4}{ }^{2-}$ & $>40$ & $\mathrm{~K}^{+}$ & 13.4 \\
\hline TC & 75 & $\mathrm{Mg}^{2+}$ & 13.82 \\
\hline TOC & 20 & $\mathrm{NH}_{4}{ }^{+}$ & 0.36 \\
\hline
\end{tabular}
$\mathrm{H}_{2} \mathrm{SO}_{4}$.

Table 4. 1 Characteristics of the WWTP effluent 
As demonstrated in Figure 4. 14, MCPA degradation in WWTPE by conventional PC method resulted in an initial $k_{\text {app }}$ value of $0.0108 \mathrm{~min}^{-1}$ and a total removal of $75.2 \%$ after 120 min treatment. When the TNA-Cu PFC system was applied, MCPA underwent more rapid degradation (1.5 times faster) with an increased initial kapp value of $0.0157 \mathrm{~min}^{-1}$ and a total removal of $87.6 \%$ after 120 min treatment. Meanwhile, an open cell voltage of around $0.12 \mathrm{~V}$ and a photocurrent of around $0.25 \mathrm{~mA}$ were obtained in the TNA-Cu PFC system when operated in WWTPE (Figure 4. 15). The obtained results suggest that applying the TNA-Cu PFC system is a facile and effective way to enhance MCPA removal from complex water matrix, and it is possible to produce electricity which can be used for other processes.

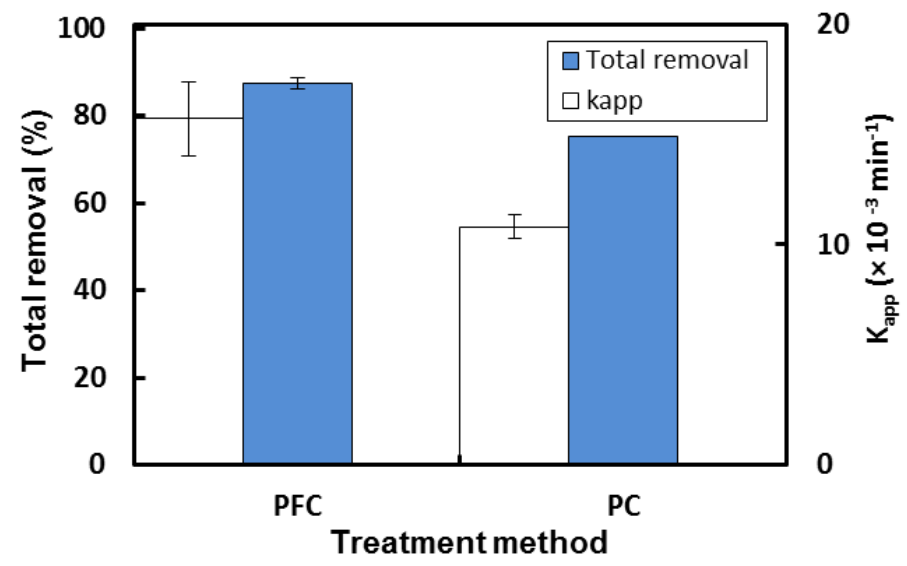

Figure 4. 14 Total removal (after $120 \mathrm{~min}$ treatment) and initial apparent degradation rate constant of MCPA in WWTP effluent by PC method and PFC method. Conditions: $[\mathrm{MCPA}]_{0}=1.0 \mathrm{mg} / \mathrm{L}, \mathrm{pH}_{0}$ adjusted to 3 by $\mathrm{H}_{2} \mathrm{SO}_{4}$. 
Chapter 4
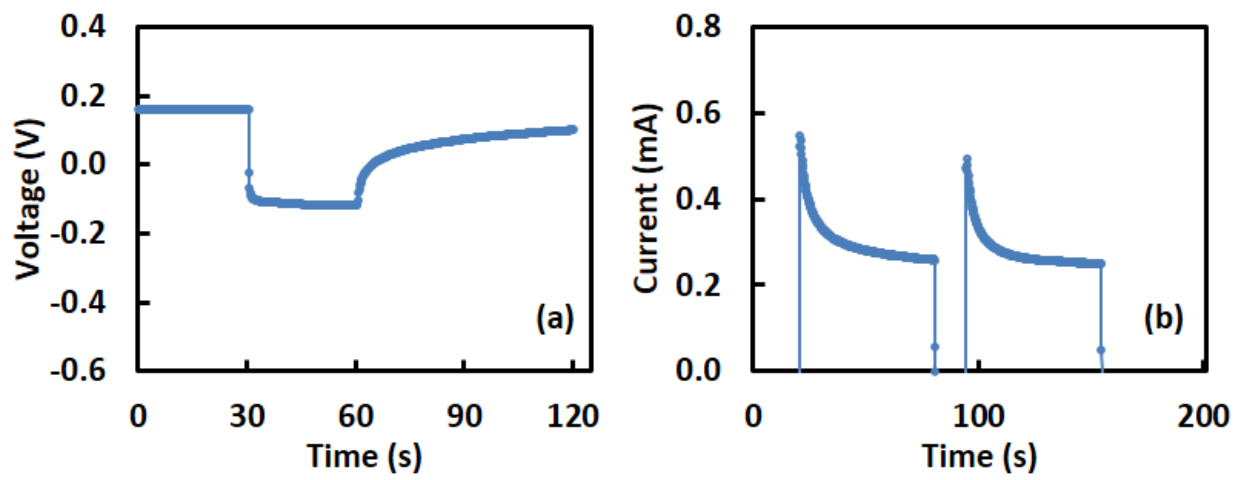

Figure 4. 15 Open cell voltage (a) and short circuit current (b) of the TNA-Cu PFC system. Conditions: $[M C P A]_{0}=1.0 \mathrm{mg} / \mathrm{L}$, in WWTP effluent, $\mathrm{pH}_{0}$ adjusted to 3 by $\mathrm{H}_{2} \mathrm{SO}_{4}$.

\subsubsection{Stability of the TNA-Cu PFC system on MCPA removal}

The stability of the TNA-Cu system was examined, in terms of its MCPA degradation performance over 6 operation cycles. For each cycle, the treatment conditions were kept identical: with initial MCPA concentration of around $1 \mathrm{mg} / \mathrm{L}$, with initial $\mathrm{pH}$ of 3 , without addition of electrolyte. Before each subsequent run, the system was washed by Milli-Q water and dried in $\mathrm{N}_{2}$ stream at ambient temperature. The results (Figure 4. 16) indicate that MCPA degradation in the TNA-Cu PFC system did not change significantly after repeated treatment cycles. The total removal of each run was nearly constant. The obtained results reveal relatively good stability and reusability of the TNA-Cu PFC system.

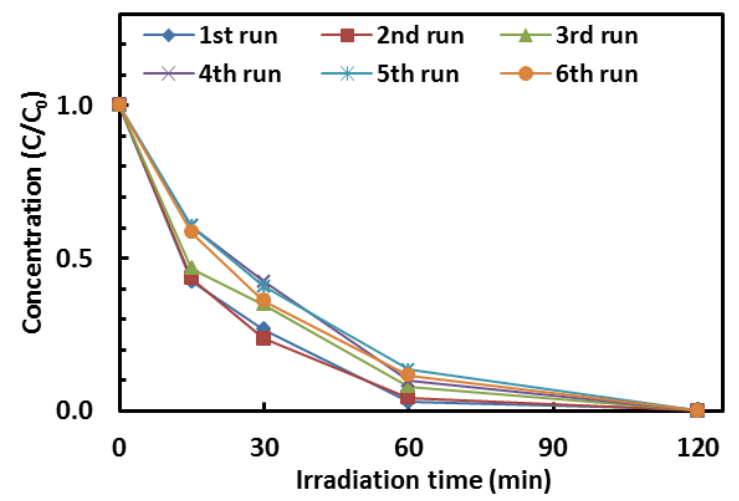

Figure 4. 16 MCPA degradation in the TNA-Cu PFC system during repeated runs.

Conditions: $[\mathrm{MCPA}]_{0}=1.0 \mathrm{mg} / \mathrm{L}, \mathrm{pH}_{0}=3$, no addition of electrolyte. 


\subsection{Conclusions}

In this study, we demonstrate that TNA-Cu PFC system is a simple and effective method to enhance MCPA photocatalytic degradation from aqueous streams compared to TNA based photocatalysis system under the same operation conditions. The main outcomes of the present work are summarized as below:

* Under the same operation conditions, enhanced MCPA removal can be obtained by simply connecting the TNA to a Cu cathode to form a PFC system, even in tap water samples and wastewater treatment plant effluent.

* Acidic pH is a crucial operational parameter of the TNA-Cu PFC system: the enhancement of MCPA degradation in the TNA-CU PFC system was largely promoted under acidic conditions.

* The enhancement of MCPA degradation in the TNA-Cu PFC system involved better $\mathrm{e}^{-} / \mathrm{h}^{+}$separation and generation of other oxidants: in the TNA-Cu PFC system, contribution of other oxidants like $\cdot \mathrm{O}_{2}^{-}, \mathrm{H}_{2} \mathrm{O}_{2}, \cdot \mathrm{HO}_{2}$ increased.

* For the acidic TNA-Cu PFC system studied in the present work, conductivity of the reaction solution was not a limiting factor. Even without addition of extra supporting electrolyte, enhanced MCPA removal can be obtained in the acidic TNA-Cu PFC system.

* Commonly present background water constituents like NOMs, nitrate ions, chloride ions, and calcium ions impose no detrimental effect on the MCPA degradation in the acidic TNA-Cu PFC system.

* The acidic TNA-Cu PFC system shows good stability over repeated treatment cycles.

\subsection{Acknowledgements}

This work was performed in the cooperation framework of Wetsus, European Centre of Excellence for Sustainable Water Technology (www.wetsus.eu). Wetsus is cofunded by the Dutch Ministry of Economic Affairs and Ministry of Infrastructure and Environment, the Province of Fryslân, and the Northern Netherlands Provinces. The authors would like to thank the participants of the research theme "Advanced water treatment" for the fruitful discussions and their financial support. The authors gratefully thank Ton van der 
Chapter 4

Zande and Mieke Kersaan-Haan for realizing the instrumental analysis, and Ernst Panstra, John Ferwerda, and Jan Tuinstra for the technical support. 
Supplementary information

Table S4. 1 Characteristics of the tap water

\begin{tabular}{ccc}
\hline Parameters & Unit & Values \\
\hline $\mathbf{C l}^{-}$ & $\mathrm{mg} / \mathrm{l}$ & 35.6 \\
$\mathrm{NO}_{2}{ }^{-}$ & $\mathrm{mg} / \mathrm{l}$ & $<0.05$ \\
$\mathrm{NO}_{3}{ }^{-}$ & $\mathrm{mg} / \mathrm{l}$ & 11.2 \\
$\mathbf{P O}^{3-}$ & $\mathrm{mg} / \mathrm{l}$ & $<0.05$ \\
$\mathrm{SO}_{4}{ }^{2-}$ & $\mathrm{mg} / \mathrm{l}$ & 0.18 \\
$\mathbf{T C}$ & $\mathrm{mg} / \mathrm{l}$ & 57.7 \\
$\mathrm{NPOC}^{-}$ & $\mathrm{mg} / \mathrm{l}$ & 4 \\
$\mathrm{IC}$ & $\mathrm{mg} / \mathrm{l}$ & 53.7 \\
$\mathrm{Ca}^{2+}$ & $\mu \mathrm{g} / \mathrm{l}$ & 27700 \\
$\mathrm{Cu}^{2+}$ & $\mu \mathrm{g} / \mathrm{l}$ & 273 \\
$\mathbf{K}^{+}$ & $\mu \mathrm{g} / \mathrm{l}$ & 2030 \\
$\mathbf{M g}^{2+}$ & $\mu \mathrm{g} / \mathrm{l}$ & 9650 \\
$\mathrm{Na}^{+}$ & $\mu \mathrm{g} / \mathrm{l}$ & 76900 \\
\hline
\end{tabular}
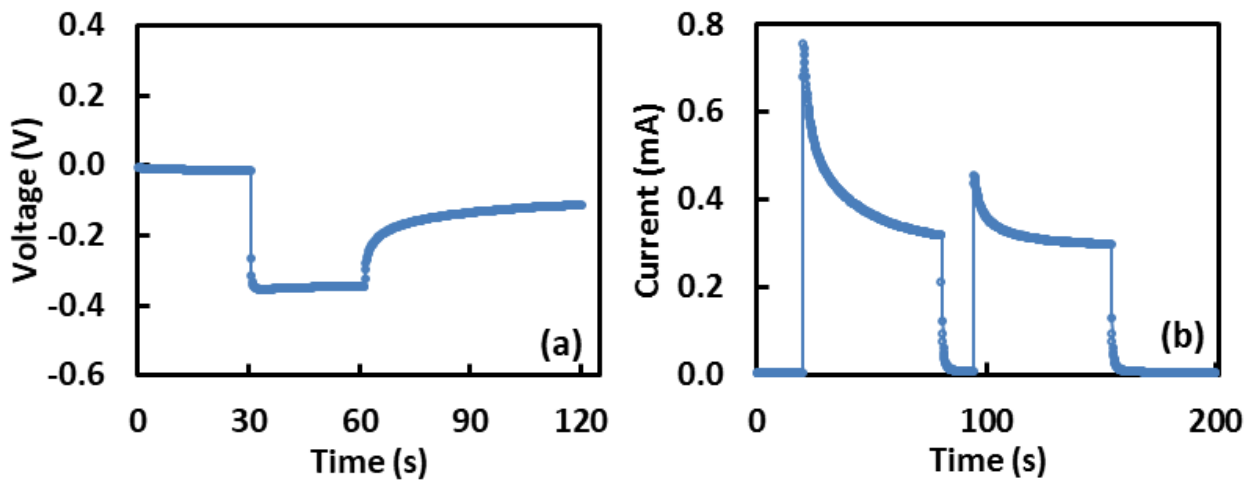

Figure S4. 1 Open cell voltage (a) and short circuit current (b) of the TNA-Cu PFC system operated in tap water. Conditions: [MCPA]0 $=1.0 \mathrm{mg} / \mathrm{L}$, in tap water. 


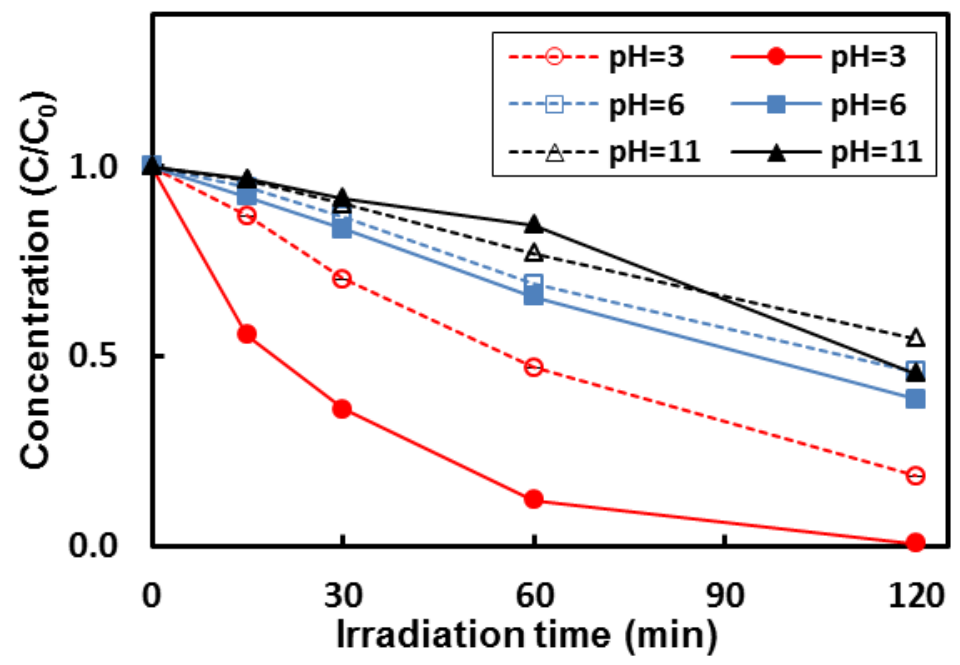

Figure S4. 2 MCPA concentration profiles during degradation by PC method (dashed line) and PFC method (solid line) at different pH levels. Conditions: [MCPA] $=1.0 \mathrm{mg} / \mathrm{L}, \mathrm{pH}$ adjusted by $\mathrm{H} 2 \mathrm{SO} 4$ or $\mathrm{NaOH}$ solutions, $0.1 \mathrm{M} \mathrm{Na2SO} 4$ as electrolyte.

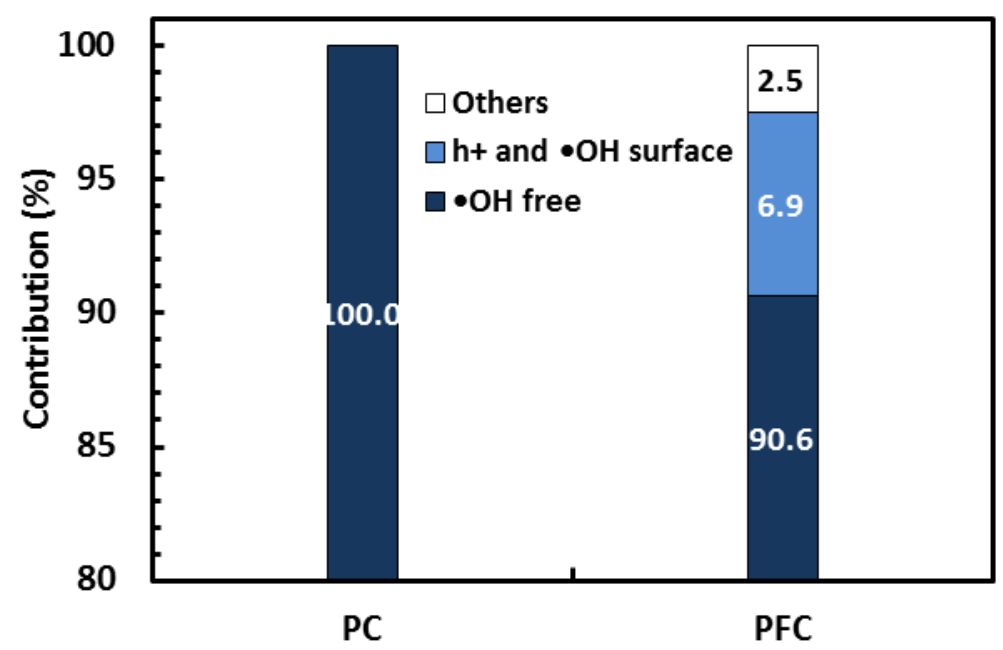

Figure S4. 3 Contribution of different reactive species on MCPA degradation by PC method and PFC method. Natural pH ( 6), [Na2SO4]=0.1 M. 
Chapter 4

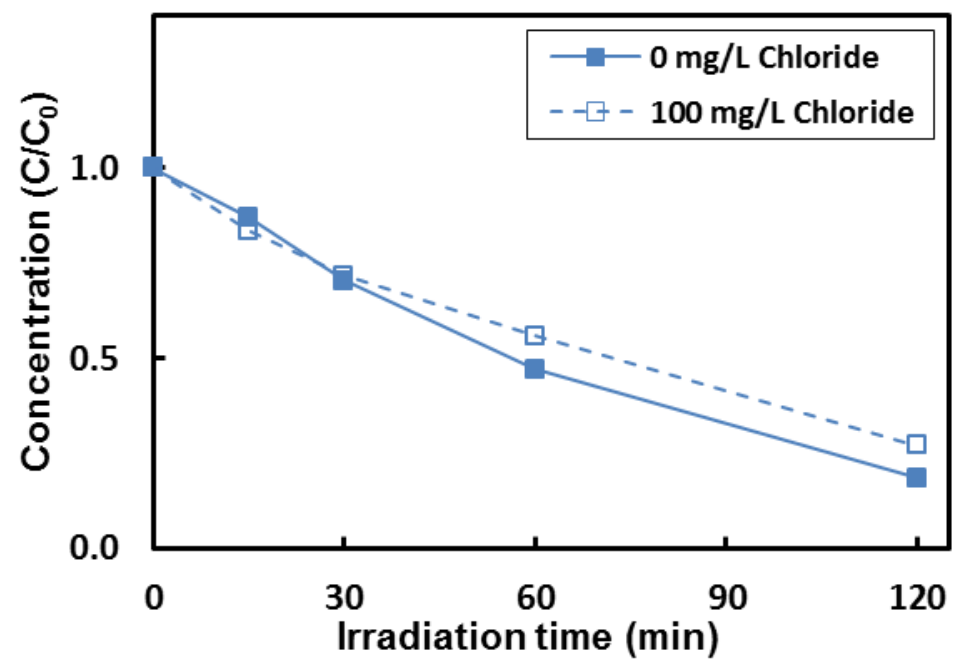

Figure S4. 4 Effect of chloride ions on the MCPA removal in the PC system. Conditions: $[\mathrm{MCPA}]_{0}=1.0 \mathrm{mg} / \mathrm{L}, \mathrm{pH}_{0}=3$. 


\section{Chapter 5}

\section{Homogeneous photosensitized degradation}

\section{of pharmaceuticals by using red light LED as}

\section{light source and methylene blue as}

\section{photosensitizer}

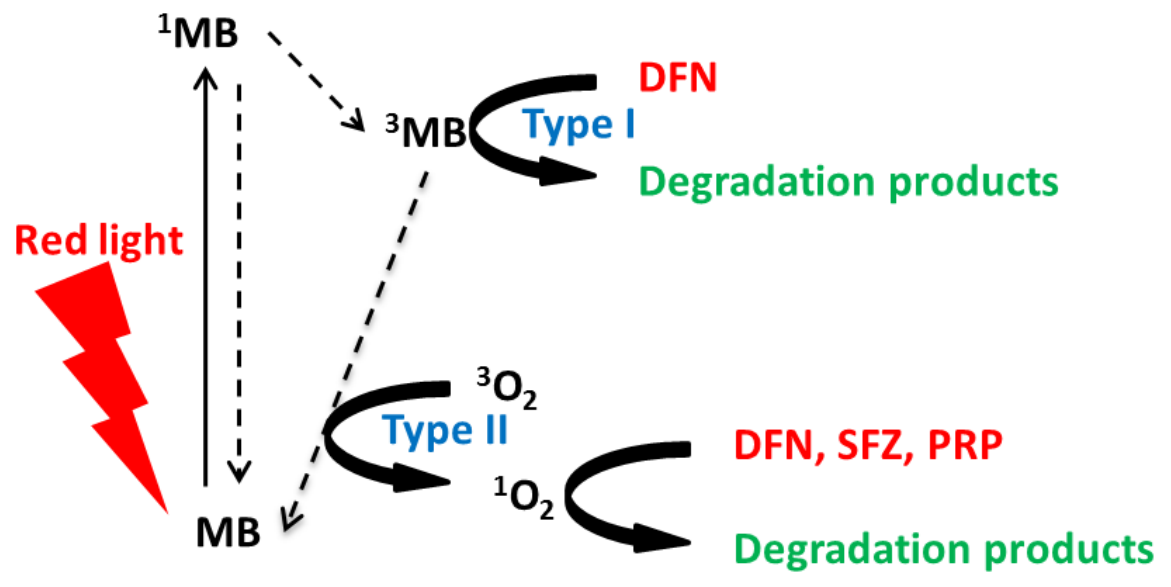

This Chapter has been published as:

Y. Ye, H. Bruning, D. Yntema, M. Mayer, H. Rijnaarts, Homogeneous photosensitized degradation of pharmaceuticals by using red light LED as light source and methylene blue as photosensitizer, Chemical Engineering Journal 316 (2017) 872-881 


\section{Chapter 5}

\section{Abstract}

Research on employing advanced oxidation processes (AOPs) for pharmaceuticals removal is gaining interests. However, detrimental effects of background water constituents in complex water matrices are limiting their implementation. In this study, we report red light induced methylene blue photosensitization ( $\mathrm{MB} /$ Red-light) as a promising alternative for pharmaceuticals removal from wastewater, because of its potential to overcome detrimental effect of background water constituents as experienced in other AOP technologies. In this study, the efficacy of $\mathrm{MB} /$ Red-light on degradation of four pharmaceutical compounds, i.e. diclofenac (DFN), metoprolol (MTP), propranolol (PRP) and sulfamethoxazole (SFZ), was investigated. The MB/Red-light photosensitization enabled degradation of three model compounds, i.e. DFN, PRP and SFZ. Degradation rates followed the order of DFN $>$ PRP $>$ SFZ. Singlet oxygen was found to be crucial in pharmaceuticals degradation, and another additional mechanism, i.e. a direct reaction with triplet $\mathrm{MB}$, also contributed to DFN degradation. The presence of two DFN degradation mechanisms were confirmed by UV-vis light absorbance spectra measurement as well as the identification of degradation products by LC-MS/MS. Effects of three common back ground water constituents were examined to assess the applicability of $\mathrm{MB} /$ red-light system in complex water matrices, which suggests that the $\mathrm{MB} /$ red-light system has great potential to be used in real wastewater. Higher $\mathrm{pH}$ was found to impose positive impact on the efficacy of the proposed system. Last but not least, red light LED is an optimum light source for the proposed MB/Red-light system, because the light emission spectrum of the LED used in this study fits well with the light absorption spectrum of the photosensitizer-methylene blue. 


\subsection{Introduction}

Various micropollutants including pharmaceuticals have been found to be present at various concentration levels in the aqueous environment [7, 14, 27]. As reported by Klavarioti, M et al. [190], they were not only detected in ground and surface water but also drinking water resources according to a broad range of studies [5-11]. Studies have indicated that the presence of pharmaceuticals in the environment imposes hazards to aquatic life at different levels, from algae to fish, even at low concentrations [27]. The problem is extending from individual compounds to mixtures of a wide range of compounds that appear nowadays in water resources at very low concentrations, and their possible synergist toxicity effects on ecological and human biological systems is not fully understood [241]. However, the presence of these chemicals in surface, ground, and drinking water resources reveals the fact that conventional wastewater treatment processes and drinking water production processes, e.g. biological processes, sedimentation, filtration, coagulation/flocculation, have insufficient efficacy in removing these wide range of compounds $[64,190]$. Therefore, more advanced treatment processes are required.

In recent years, vast efforts have been directed to develop efficient removal techniques for such purpose, such as advanced oxidation processes (AOPs) as $\mathrm{UV} / \mathrm{H}_{2} \mathrm{O}_{2}[55-$ 59], UV/TiO 2 [60-62], Fenton and Photo-Fenton[63, 83], where a strong oxidizing species hydroxyl radical is the primary oxidant. The strong oxidizing power of the hydroxyl radical exhibits great potential in various applications, including organic pollutant removal, disinfection etc. However, it is also well documented that, in relatively complex water matrixes, the background water constituents, including inorganic salts (e.g. phosphate, bicarbonate, sulphate, etc.) and natural organic matters (NOMs), impose large detrimental effect on the performance of such hydroxyl radical mediated processes [166]. For instance, in AOPs involving the use of solid phase catalysts, anions like phosphate and bicarbonate remain strongly adsorbed onto catalysts surfaces and can therefore deactivate the site where it adsorbed $[96,166]$. Furthermore, inorganic anions like phosphate and bicarbonate are also able to act as hydroxyl radical scavengers. A study conducted by J. Brame et al. [177] reported that the presence of phosphate ions even at low concentrations could cause significant decrease in the performance of $\mathrm{UV} / \mathrm{H}_{2} \mathrm{O}_{2}$ process, which was attributed to 


\section{Chapter 5}

scavenging of hydroxyl radicals by phosphate ions. NOMs interfere with performance of various existing AOPs by two main mechanisms. The first one is absorption of UV light, which is well known as "inner filter effect". NOMs are usually able to absorb light in the UV and near-UV range. The presence of NOMs in water would decrease the availability of UV light for semiconductors $/ \mathrm{H}_{2} \mathrm{O}_{2}$ and any other species needed to produce hydroxyl radicals, in the cases of all UV related AOPs. The other reason that NOM could reduce the performance of most existing AOPs is that NOMs can act as hydroxyl radical scavenger. In the same study conducted by J. Brame et al. [177], significant inhibitory effect of NOM on the degradation of pharmaceuticals in $\mathrm{UV} / \mathrm{H}_{2} \mathrm{O}_{2}$ process was reported. Viewing the aforementioned facts, recently studies have been carried out to investigate the efficacy of employing photosensitization induced by visible light and solid photosensitizers for organic chemical removal in various water matrices $[64,129,130]$, where singlet oxygen $\left({ }^{1} \mathrm{O}_{2}\right)$ was considered to be the primary oxidant. ${ }^{1} \mathrm{O}_{2}$ is an energetically rich form of molecular oxygen, which has about $1 \mathrm{eV}$ higher energy and is more reactive than ground state oxygen[127]. As an oxidant which has a higher oxidizing potential (about $1 \mathrm{~V}$ higher [127]) than ground state oxygen, its application in organic pollutant elimination has been investigated [178-181]. However, involvement of solid photosensitizers is still a drawback. Because ${ }^{1} \mathrm{O}_{2}$ has a very short life time, the presence of ${ }^{1} \mathrm{O}_{2}$ is limited to a thin layer close to the solid phase; only adsorbed pollutants can be well degraded [182]. Moreover, inorganic ions and NOMs in water matrices can still reduce the process efficiency by blocking the active site of solid sensitizers. In this manner, homogeneous singlet oxygen mediated processes can be an alternative, because the aforementioned problems associated with heterogeneous system can be avoided. Methylene blue (MB) has great potential to be utilized as homogeneous photosensitizer for such purpose. It has strong light absorption in visible range which can avoid the inner filter effect of NOMs in complex water matrices, has a relatively high quantum yield of singlet oxygen formation [183], and has a long history of safe use in human treatment without major side effect. The red light induced MB photosensitization process for singlet oxygen formation is reported to have a broad application field including disinfection in not only wastewater treatment but also blood products [184-189], where singlet oxygen was generated to kill pathogens, as well as medical therapy, where red light induced MB photosensitized singlet oxygen was directed to selectively kill tumour cells for 
cancer therapy [187]. Nevertheless, no attempt has been made to employ $M B$ as homogeneous photosensitizer for pharmaceuticals removal.

Light source is another important factor for photon-involved processes, because it imposes great influence on the operational and maintenance costs. Conventional light sources include mercury lamps (for UV involved systems), fluorescent lamps and Xe-arc lamps (for visible light involved systems), etc. Recently, light emitting diodes (LED) are gaining increasing interests in view of their advantages: short warm-up time, no hazardous materials disposal, long life time, compatibility, narrow light emission spectra, etc [62, 242]. Attempt has been made to utilize LED as light sources in photo-oxidation processes very recently $[79,242,243]$. However, to the best of the authors` knowledge, no studies have been conducted to investigate the use of red light LED for photodegradation of pharmaceuticals.

In the present study, the overall objective is to investigate the feasibility of applying red light LED as light source and MB as photosensitizer for photodegradation of pharmaceuticals. The model compounds in this study include diclofenac (DFN), sulfamethoxazole (SFZ), metoprolol (MTP), and propranolol (PRP). They are classified in the group of pharmaceuticals most commonly present in the environment [7], and have diverse chemical structures. The degradation kinetics of the model compounds was presented. The degradation mechanisms were discussed. Effects of different background water constituents, i.e. phosphate ions, bicarbonate ions and NOM, were studied to assess the applicability of the proposed method in real life application. Effect of $\mathrm{pH}$ on the efficacy of $\mathrm{MB} /$ Red-light system was also studied.

\subsection{Materials and methods}

\subsubsection{Materials}

Diclofenac (DFN), sulfamethoxazole (SFZ), metoprolol (MTP), and propranolol (PRP) were purchased from Sigma-Aldrich (Germany) and were used as received. Stock solutions of each pharmaceutical compound were prepared at concentrations of $200 \mathrm{mg} / \mathrm{L}$ for each of these model compounds. $\mathrm{Na}_{2} \mathrm{CO}_{3}$ and $\mathrm{NaHCO}_{3}$ were purchased from VWR (Belgium) and were used as received. $\mathrm{Na}_{2} \mathrm{HPO}_{4}$ and $\mathrm{NaH}_{2} \mathrm{PO}_{4}$ were purchased from Boom BV (The 


\section{Chapter 5}

Netherlands) and were used as received. Pony Lake (Antarctica) Fulvic Acid Reference natural organic matter (NOM) was obtained from the International Humic Substances Society (USA) and a NOM stock solution at $100 \mathrm{mg} / \mathrm{L}$ was prepared. The photosensitizer methylene blue was purchased from Boom BV (The Netherlands) and a MB stock solution at $200 \mathrm{mg} / \mathrm{L}$ was prepared. Ultrapure water from a Milli-Q Advantage A10 system (Merck Millipore, Darmstadt, Germany) was used for preparation of all stock solutions and reaction solutions.

\subsubsection{Red-light LED and experimental setup}

The red-light LED (LZ4-00R200) was purchased from LED-ENGIN, Inc. USA. Typical light emission of the LED is in the range from around $600 \mathrm{~nm}$ to $700 \mathrm{~nm}$, with peak wave length in between $655 \mathrm{~nm}$ and $670 \mathrm{~nm}$. The red-light LED was mounted onto a cooling plate for heat dissipation, and attached a lens to concentrate the light irradiation and avoid direct contact of the LED with the reactor to prevent heating up of the reaction solution. The LED was powered by an EST150 DC power supply (Delta Elektronika, The Netherlands). The input current of the LED was set to be $0.8 \mathrm{~A}$, which gave an input electrical power of $7.7 \mathrm{~W}$. The red-light LED was placed under the bottom of the reactor. The radiant power density entering the reactor was measured to be $1.77 \mathrm{~W}$, by using a THORLABS S120C photodiode power sensor (THORLABS, USA). Under the applied irradiation conditions, the photon flux was calculated to be around $9.77 \mu \mathrm{mol} / \mathrm{s}$.

A glass cylinder was used as reactor ( $270 \mathrm{~mm}$ height and $60 \mathrm{~mm}$ diameter). The reactor was wrapped with aluminium paper to avoid interference of ambient light. (Figure S5. 1 in the supplementary information shows the schematic of the set-up.)

\subsubsection{Photodegradation experiments}

Experiments were divided into different groups. The group name and experimental conditions of photochemical experiments can be found in Table 5. 1. Pharmaceutical stock solutions were spiked into ultrapure water (MilliQ) to achieve the desired initial pharmaceutical concentrations $(1.0 \mathrm{mg} / \mathrm{L})$ in reaction solutions $(500 \mathrm{~mL})$. Stock MB solution was added to the reaction solution to achieve initial MB concentration of $1.5 \mathrm{mg} / \mathrm{L}$, except for Group B where no MB was added. Photochemical experiments were carried out at 
ambient temperature. Except the experiments investigating effect of $\mathrm{pH}$ (phosphate buffer system was used to achieve and maintain the designated $\mathrm{pH}$ ), the $\mathrm{pH}$ of all other experiments was unadjusted and circum-neutral, with $\mathrm{pH}$ value in the range of 6-7, and no significant change in $\mathrm{pH}$ was observed during reaction. $\mathrm{N}_{2}$ or air was continuously supplied from the bottom of the reactor, to maintain the desired dissolved oxygen (DO) concentration and to facilitate mixing. The DO concentration of the reaction solution was monitored using a DO probe (Oxymax COS22/22D, Endress+Hauser). With continuous air supply, the reaction solutions were maintained air-equilibrated, while with $\mathrm{N}_{2}$ supply the DO was maintained at $0 \mathrm{mg} / \mathrm{L}$. During the experiments, $5 \mathrm{~mL}$ samples were taken at designated times and the total irradiation time of each run was 120 minutes. All experiments were carried out in duplicates.

Table 5. 1 Overview of the experimental conditions of photodegradation experiments

\begin{tabular}{ccccc}
\hline Group name & Pharmaceuticals & MB & Red-light & Gas supply \\
\hline A & DFN, MTP,PRP, SFZ & + & ON & Air \\
B & DFN, MTP,PRP, SFZ & - & ON & Air \\
C & DFN, MTP,PRP, SFZ & + & OFF & Air \\
D & DFN, MTP,PRP, SFZ & + & ON & $\mathrm{N}_{2}$ \\
E & - & + & ON & $\mathrm{N}_{2}$ \\
F & DFN & + & ON & Air \\
G & DFN & + & ON & $\mathrm{N}_{2}$ \\
\hline
\end{tabular}

\subsubsection{Singlet oxygen quenching experiments}

Singlet oxygen quenching experiments were conducted to study the role of ${ }^{1} \mathrm{O}_{2}$ in photodegradation of model compounds in the $\mathrm{MB} /$ Red-light system. A well-known ${ }^{1} \mathrm{O}_{2}$ quencher sodium azide $\left(\mathrm{NaN}_{3}\right)$ was added to reaction solutions $(0.5 \mathrm{~g} / \mathrm{L})$. All other experimental conditions and procedures were identical with Group A. 


\section{Chapter 5}

\subsubsection{Effect of background water constituents}

Three common water constituents, i.e. phosphate ions, bicarbonate/carbonate ions, and NOM, were used to study their effect on performance of MB/Red-light system. Effects of these chemicals were studied individually. The $\mathrm{pH}$ was circum-neutral in the range of 6 to 7. All other experimental conditions were kept identical with Group A.

\subsubsection{Effect of $\mathrm{pH}$}

Experiments were carried out to investigate the influence of solution $\mathrm{pH}$ on the efficacy of photosensitized degradation of pharmaceuticals, with $\mathrm{pH}$ of 4.6 (acidic condition), 7 (neutral condition), and 9 (alkaline condition). The initial MB concentration of $1.5 \mathrm{mg} / \mathrm{L}$ was applied, the initial pharmaceutical concentrations were kept at $1.0 \mathrm{mg} / \mathrm{L} .50 \mathrm{mM} \mathrm{H}_{2} \mathrm{PO}_{4}^{-}$ $/ \mathrm{HPO}_{4}{ }^{2-}$ buffer system was used to achieve and maintain designated $\mathrm{pH}$. The experiments were conducted under air equilibrated condition.

\subsubsection{Analytical methods}

Absorption spectra of samples in the wavelength range from $250 \mathrm{~nm}$ to $700 \mathrm{~nm}$ were measured with a UV/Vis spectrophotometer (Shimadzu UV-1800). Total organic carbon (TOC) level was measured for Group $A$ to examine the efficacy of $M B /$ red-light system in mineralization of pharmaceuticals, by a TOC-L CPH analyser equipped with an ASI$\mathrm{L}$ autosampler, as described elsewhere [70]. For pharmaceuticals concentrations, samples were analysed using an Agilent LC-MS/MS system consisting of Agilent infinity 1260 LCsystem (degasser, binary pump, auto sampler with cooled tray and column oven) and Agilent 6420 triple Quadrupole Mass Spectrometer with Electrospray ion source. The compounds were separated using a Phenomenex Gemini Phenyl-Hexyl column (150x3 mm, particle size $5 \mu \mathrm{m}$ ) and a gradient of acetonitrile ( 5 to $90 \%$ ) and Ammonium Formate buffer in water. The compounds were detected and quantified on the 6420-QQQ-MS using compound specific multiple Dynamic MRM transitions. Detailed information of the analytical method used can be found in literature [220]. 


\subsection{Results and discussion}

\subsubsection{Degradation of pharmaceutical compounds by $M B /$ Red-light} photosensitization

Two groups of photochemical experiments were conducted: (Group A) photosensitized degradation in the presence of $M B$ and red light, under air-equilibrated conditions; and (Group B) direct photolysis under red light irradiation. Dark control experiments (Group C) were carried out to evaluate the stability of the solutions and to rule out the loss of model compounds by physical processes like stripping and adsorption. Experiments with each individual model compound were also performed to exclude the possible competition among the model compounds, and the results show no significant difference (Figure S5. 2).

Table 5. 2 Time-based pseudo-first-order kinetic constants and total removal of pharmaceuticals after $120 \mathrm{~min} \mathrm{MB/Red-light} \mathrm{treatment}$

\begin{tabular}{cccc}
\hline Compound & $\mathbf{k}_{\text {app }}\left(\mathbf{m i n}^{-1}\right)$ & $\mathbf{R}^{\mathbf{2}}$ & Total removal \\
\hline Diclofenac & 0.0572 & 0.9958 & $98.94 \%$ \\
Propranolol & 0.0170 & 0.9904 & $79.98 \%$ \\
Sulfamethoxazole & 0.0022 & 0.9896 & $15.70 \%$ \\
Metoprolol & 0.0003 & 0.6661 & $0.23 \%$ \\
\hline
\end{tabular}

The concentrations $\left(C / C_{0}\right)$ of model compounds as a function of irradiation time were plotted as shown in Figure 5. 1. The results demonstrate that three of the model compounds were degraded via photosensitized degradation. The total removal and kinetics rate constants were found to vary among the different compounds (see Figure 5. 1a, and Table 5. 2). Degradation of pharmaceuticals in the proposed MB/Red-light system fits the pseudo-first-order kinetic model, but to avoid confounding effects of degradation products, only initial degradation rate constants were examined in this study. Only initial degradation data (0 - $15 \mathrm{~min}$ ) was used to calculate time-based pseudo-first order rate constants ( $k_{\text {app }}$ ) in this study [177]. The applied conditions enabled rapid degradation of DFN and PRP (with 


\section{Chapter 5}

time-based pseudo-first order rate constant $k_{a p p}$ of $0.0572 \mathrm{~min}^{-1}$ and $0.0170 \mathrm{~min}^{-1}$, respectively), with total removal of $98.94 \%$ and $79.98 \%$ after 120 min treatment, respectively. Degradation of SFZ was much slower $\left(k_{a p p}=0.0022 \mathrm{~min}^{-1}\right)$, with a total removal of $15.70 \%$. No detectible degradation of MTP was obtained under the applied conditions.

Furthermore, the UV-vis spectra of the reaction solution at different times were determined as shown in Figure 5. 2. The results show that the UV-vis spectrum of reaction solution changed significantly after 120 min irradiation of red light. The spectrum curve at 0 min shows two major peaks in both the UV range (attributed to the presence of MB and pharmaceuticals) and the red light range (attributed to the presence of $M B$ ). Both peaks decreased dramatically after $120 \mathrm{~min}$, reflecting the degradation of pharmaceuticals and MB. Light absorption in the range of $300 \mathrm{~nm}$ to $550 \mathrm{~nm}$ experienced slight increase, revealing formation of some degradation products. Previous studies on singlet oxygen mediated micropollutant removal have pointed out that singlet oxygen mediated processes do not lead to total mineralization of the target pollutants [129], which is in accordance with our results of TOC measurement (Figure S5. 3), but it should also be noticed that even uncomplete oxidation of target pollutants by singlet oxygen often lead to drastic reduction in their biological or estrogenic activities [129].

Model compounds were exposed to red light irradiation, so direct photolysis might also contribute to their degradation. However, the results of Group B (shown in Figure 5. 1b) clearly rule out the contribution of direct photolysis on degradation of the pharmaceuticals: without addition of $\mathrm{MB}$, none of the model compounds were degraded under red light irradiation. This can be attributed to the fact that the model compounds in this study have no light absorption in the red light range (Figure S5. 4). Moreover, results of dark control experiments (shown in Figure 5. 1c) showed no change in model compounds concentrations. Hence, the degradation of model compounds was subjected to red light induced MB photosensitization process. 
Chapter 5
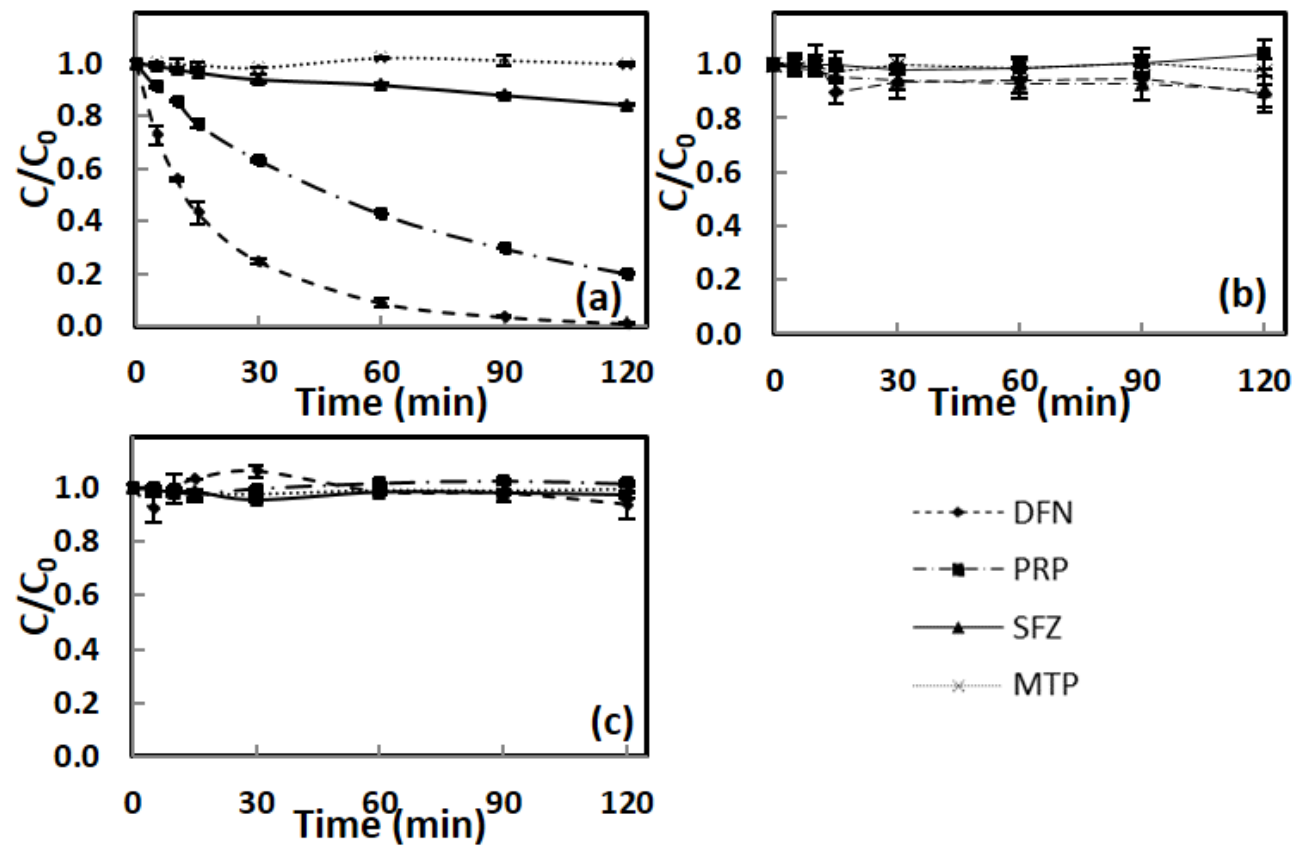

Figure 5. 1 Pharmaceutical compounds degradation profile under different conditions as a function of time: (a) Group A, (b) Group B, (c) Group C.

$[D F N]_{0}=[M T P]_{0}=[P R P]_{0}=[S F Z]_{0}=1.0 \mathrm{mg} / \mathrm{L},[\mathrm{MB}]_{0}=1.5 \mathrm{mg} / \mathrm{L}$ (except Group B), pH unadjusted.

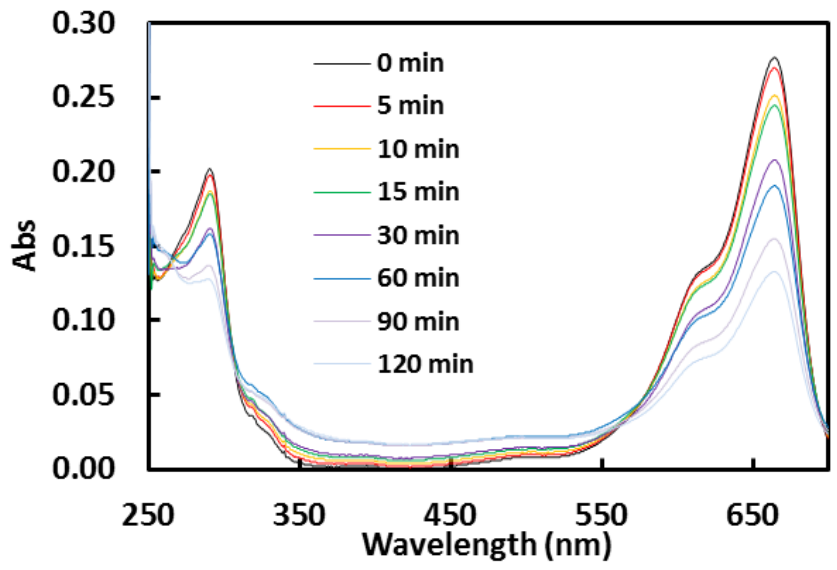

Figure 5. 2 Absorption spectra change observed in Group A. 


\section{Chapter 5}

\subsubsection{Crucial role of singlet oxygen in degradation of propranolol and sulfamethoxazole}

The possible mechanisms of the red-light induced MB photosensitization has been proposed elsewhere [186, 244]. MB molecule can be activated to the excited singlet state by absorbing light (mainly in red light range as shown in Figure S5. 5), as reported by L.S. Peloi et al. [187]. The generated singlet state of MB is unstable and can rapidly either decay to its ground state or give the excited triplet state $\mathrm{MB}\left({ }^{3} \mathrm{MB}\right)$, with comparable probability. ${ }^{3} \mathrm{MB}$ can react with dissolved oxygen (DO) to form ${ }^{1} \mathrm{O}_{2}$ (energy transfer, $\sim 10^{9} \mathrm{M}^{-1} \mathrm{sec}^{-1}$ )[186] or superoxide anion radical (electron transfer, $<10^{7} \mathrm{M}^{-1} \mathrm{sec}^{-1}$ )[186] which is defined as Type II photosensitization; alternatively ${ }^{3} \mathrm{MB}$ directly reacts with substrates via electron transfer, which is defined as Type I photosensitization.

Experiments (Group D) were carried out to investigate the involvement of DO in degradation of the model compounds, and the experimental conditions were identical with that of Group A except that the gas supply was switched to $N_{2}$. With continuous $N_{2}$ supply, the DO concentration was maintained at $0 \mathrm{mg} / \mathrm{L}$. Therefore the Type II photosensitization pathway was completely inhibited because of the absence of oxygen in the liquid phase as energy acceptor for ${ }^{1} \mathrm{O}_{2}$ formation or as electron acceptor for superoxide anion radical formation. The results (as shown in Figure 5. 3) indicate that degradations of PRP and SFZ are dependent on the presence of DO, suggesting that DO plays a crucial role in their degradation.

As aforementioned, $\mathrm{DO}$ in the $\mathrm{MB} /$ Red-light can participate the degradation processes via two possible routes, thus formation of superoxide radical via electron transfer with excited $\mathrm{MB}$ molecules or formation of ${ }^{1} \mathrm{O}_{2}$ via energy transfer with excited $\mathrm{MB}$. In the presence of molecular oxygen, the production of ${ }^{1} \mathrm{O}_{2}$ is approximately 100 times faster [186] compared with production of superoxide anion. Studies have reported that in methylene blue photosensitization, in the presence of oxygen, the involvement of the superoxide anion radical is only a side-reaction, while the ${ }^{1} \mathrm{O}_{2}$ mediated oxidation is usually the dominant pathway [245, 246]. Results of ${ }^{1} \mathrm{O}_{2}$ quenching experiments (Figure 5. 4) support the hypothesis that ${ }^{1} \mathrm{O}_{2}$ is the dominant oxidizing species for degradation of PRP and SFZ: with addition of $0.5 \mathrm{~g} / \mathrm{L} \mathrm{NaN}_{3}$ (a well-known singlet oxygen quencher), degradation of PRP and 
SFZ experienced drastic decrease. Therefore, it can be concluded that DO is a crucial factor in degradation of PRP and SFZ in the $\mathrm{MB} /$ Red-light process, and ${ }^{1} \mathrm{O}_{2}$ is the dominant reactive oxygen species (ROS) responsible for their degradation.

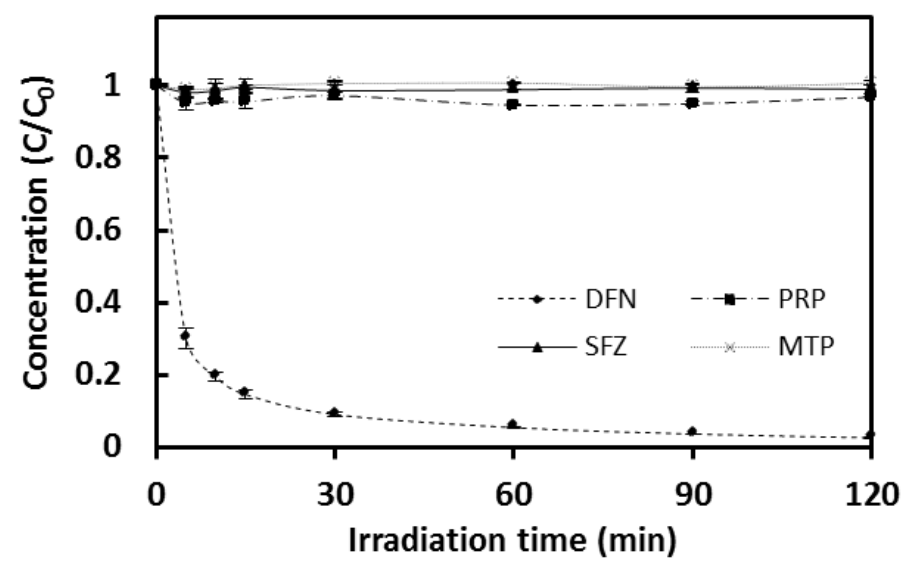

Figure 5. 3 Pharmaceutical compounds degradation profile as a function of time under anaerobic conditions (Group D). $[\mathrm{DFN}]_{0}=[\mathrm{MTP}]_{0}=[\mathrm{PRP}]_{0}=[\mathrm{SFZ}]_{0}=1.0 \mathrm{mg} / \mathrm{L},[\mathrm{MB}]_{0}=1.5$ $\mathrm{mg} / \mathrm{L}, \mathrm{pH}$ unadjusted.

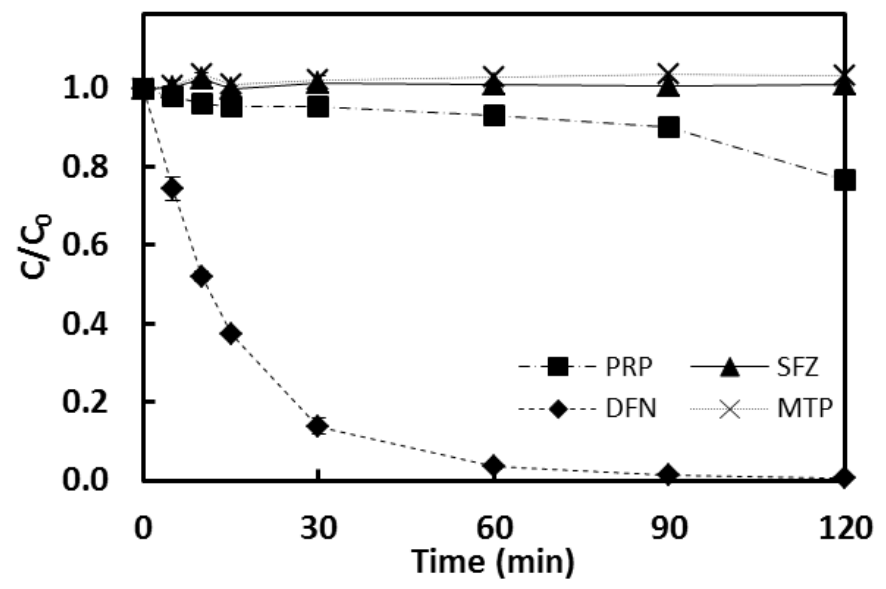

Figure 5. 4 Effect of addition of singlet oxygen quencher $(0.5 \mathrm{~g} / \mathrm{L} \mathrm{NaN})$ on photosensitized degradation of model compounds. $[\mathrm{DFN}]_{0}=[\mathrm{MTP}]_{0}=[\mathrm{PRP}]_{0}=[\mathrm{SFZ}]_{0}=1.0$ $\mathrm{mg} / \mathrm{L},[\mathrm{MB}]_{0}=1.5 \mathrm{mg} / \mathrm{L}, \mathrm{pH}$ unadjusted. 


\section{Chapter 5}

\subsubsection{Degradation mechanisms of diclofenac}

\subsubsection{Involvement of Type I photosensitization for diclofenac degradation}

Unlike SFZ and PRP, rapid degradation of DFN took place even when DO concentration is $0 \mathrm{mg} / \mathrm{L}$ (Figure 5. 3). Combining the fact that quenching of ${ }^{1} \mathrm{O}_{2}$ did not cause any noticeable decrease in its degradation (Figure 5. 4), it is clear that Type I photosensitization which involves direct reaction between the photosensitizer $\mathrm{MB}$ and target pollutants can also contribute to degradation of pharmaceuticals, depending on the properties of specific pharmaceuticals. Theoretically, the photochemical properties of MB enable non- ${ }^{1} \mathrm{O}_{2}$ reaction route. In addition to Type II photosensitization involves DO, when the triplet excited state of MB is formed, it can also undergo Type I photosensitization by directly reacting with substrates, DFN in this case, through electron transfer. Therefore ${ }^{1} \mathrm{O}_{2}$ is not compulsory for the photosensitized degradation of DFN, thus Type I photosensitization is also of importance for DFN degradation.

Under anaerobic conditions, MB itself is non-degradable under red light irradiation (Figure S5. 6), supporting the hypothesis that DFN degradation under anaerobic conditions resulted from the reaction between $\mathrm{MB}$ and DFN. Under air-equilibrated conditions, the degradation of DFN follow pseudo-first-order reaction with a good correlation factor $\left(R^{2}=0.997\right)$ (Figure 5. 1a), and the decreased MB concentration (Figure S5. 7) did not cause significant change in the specific DFN degradation rate, as reaction proceeded; while under anaerobic conditions DFN degradation rate was largely affected (Figure 5. 3) by the decreased MB concentration (Figure S5. 7).

Moreover, the results suggest that when DO is present in the reaction solution, under the applied conditions, the ${ }^{1} \mathrm{O}_{2}$ mediated pathway is the dominant pathway responsible for DFN degradation. The ${ }^{1} \mathrm{O}_{2}$ mediated type II pathway is slower than the type I photosensitization which involves direct electron transfer between ${ }^{3} \mathrm{MB}$ and DFN; when no molecular oxygen is available as energy acceptor to quench ${ }^{3} \mathrm{MB}$, Type I photosensitization becomes the primary reaction pathway for DFN. One research [246] reported the same finding that electron transfer mechanism might be much faster than ${ }^{1} \mathrm{O}_{2}$ mediated pathway. The initial reaction rate in the absence of DO was much higher than the initial reaction rate in DO saturated condition, but the difference in specific reaction rate 
decreased during the process because of decreasing specific reaction rate when [DO] $=0$ $\mathrm{mg} / \mathrm{L}$. The total removal for DFN after $2 \mathrm{~h}$ reaction was the same when [DO] $=0 \mathrm{mg} / \mathrm{L}$ and DO was maintained saturated. With increasing irradiation time, decreased MB concentrations were observed in both runs (Figure S5. 7). The decreased difference in reaction rate could be attributed to the decreased $M B$ concentration. On the one hand, under the applied air-equilibrated conditions, ${ }^{1} \mathrm{O}_{2}$ mediated pathway was predominant in DFN degradation, and its reaction rate was not affected by the decreased MB concentration because the ${ }^{1} \mathrm{O}_{2}$ production remained sufficient even at the decreased $\mathrm{MB}$ concentration; on the other hand, under the applied conditions when [DO] $=0 \mathrm{mg} / \mathrm{L}$, the Type I photosensitization reaction was subjected to not only the DFN concentration but also the $\mathrm{MB}$ concentration, and its reaction rate decreased when $\mathrm{MB}$ concentration decreased. With lower MB concentrations, less ${ }^{3} \mathrm{MB}$ could be produced, and thus less ${ }^{3} \mathrm{MB}$ was available for oxidative degradation of DFN.

${ }^{1} \mathrm{O}_{2}$ mediated pathway and Type I photosensitization are parallel pathways for $\mathrm{MB} /$ Red-light photosensitized degradation of DFN. DO concentration plays an import role determining the involvement of each reaction pathway. The research using pyrylium salt as photosensitizer conducted by Miranda et al., (2000)[246] found that the electron transfer pathway can be enhanced by oxygen. However, in our research, contradicting results were obtained. On the contrary to pyrylium salt photosensitized process, electron transfer pathway (Type I photosensitization) appears to be negatively affected by oxygen: under the applied conditions, a saturated $\mathrm{DO}$ condition made ${ }^{1} \mathrm{O}_{2}$ mediated pathway the dominant pathway in the degradation of DFN, while a DO concentration of $0 \mathrm{mg} / \mathrm{L}$ made Type I photosensitization to be the dominant. One hypothesis could be that, under aerobic conditions, the DO molecules can rapidly quench energy from ${ }^{3} \mathrm{MB}$ to form ${ }^{1} \mathrm{O}_{2}$, resulting in much less available ${ }^{3} \mathrm{MB}$ for direct reaction with DFN. In addition, reaction between DFN and ${ }^{1} \mathrm{O}_{2}$ is slower than the direct reaction between ${ }^{3} \mathrm{MB}$ and $\mathrm{DFN}$, so the overall DFN degradation rate was reduced in the presence of $D O$. 


\section{Chapter 5}

\subsubsection{Difference between diclofenac degradation products formation under aerobic conditions and anaerobic conditions}

To further confirm the presence of two different degradation routes of DFN, $\mathrm{MB} /$ Red-light photosensitized DFN degradation experiments were conducted under aerobic conditions (air-equilibrated, Group F) and anaerobic conditions (Group G) respectively. The results show that, after $120 \mathrm{~min}$, light absorption spectrum of the aerobic reaction solution (Figure 5. 5b) differed significantly from that of the anaerobic reaction solution (Figure 5. $5 a)$. In the wavelength range of $300 \mathrm{~nm}$ to $500 \mathrm{~nm}$, light absorption of the aerobic reaction solution slightly increased after treatment (comparing to Figure 5. $5 \mathrm{c}$ and Figure 5. 5d), revealing the formation of degradation products which has light absorption in this range, whereas light absorption of the anaerobic reaction solution decreased significantly to near baseline. This is a clear indication of the presence of two different routes for DFN degradation.

Degradation products (DP) of DFN under aerobic conditions (air-equilibrated, Group F) and anaerobic conditions (Group G) were investigated by LC-MS/MS. The results show that under aerobic conditions, several degradation products with different $\mathrm{m} / \mathrm{z}$ ratios were observed at different retention times (RT): three DPs with the same $\mathrm{m} / \mathrm{z}$ ratio of 202 were observed at RT of $1.40 \mathrm{~min}, 1.73 \mathrm{~min}$ and $1.83 \mathrm{~min}$ respectively; one DP with $\mathrm{m} / \mathrm{z}$ of 232 and RT of $6.27 \mathrm{~min}$; one DP with $\mathrm{m} / \mathrm{z}$ of 234 and RT of $2.09 \mathrm{~min}$; one DP with $\mathrm{m} / \mathrm{z}$ of 247 and RT of 7.82; one DP with $\mathrm{m} / \mathrm{z}$ of 250 and RT of 6.49 ; two DPs with the same $\mathrm{m} / \mathrm{z}$ ratio of 260 were observed at different RT of $5.67 \mathrm{~min}$ and $6.70 \mathrm{~min}$ respectively; one DP with $\mathrm{m} / \mathrm{z}$ of 262 and RT of 6.19; five DPs with the same $\mathrm{m} / \mathrm{z}$ ratio of 266 were observed at RT of 5.02 min, $5.14 \mathrm{~min}, 6.92 \mathrm{~min}, 7.10 \mathrm{~min}$ and $9.19 \mathrm{~min}$, respectively. It should be pointed out that due to lack of more advanced apparatus, exact structures of the observed DPs could not be confirmed herein, but tentative structures of DPs were assigned by comparing our findings with literature. Tentative structures of some of those degradation products were assigned and shown in Figure 5. 6. These transformation products are in agreement with previous studies where singlet oxygen was reported to be predominant oxidant $[6,241]$, suggesting the involvement of singlet oxygen in diclofenac degradation under aerobic conditions. 

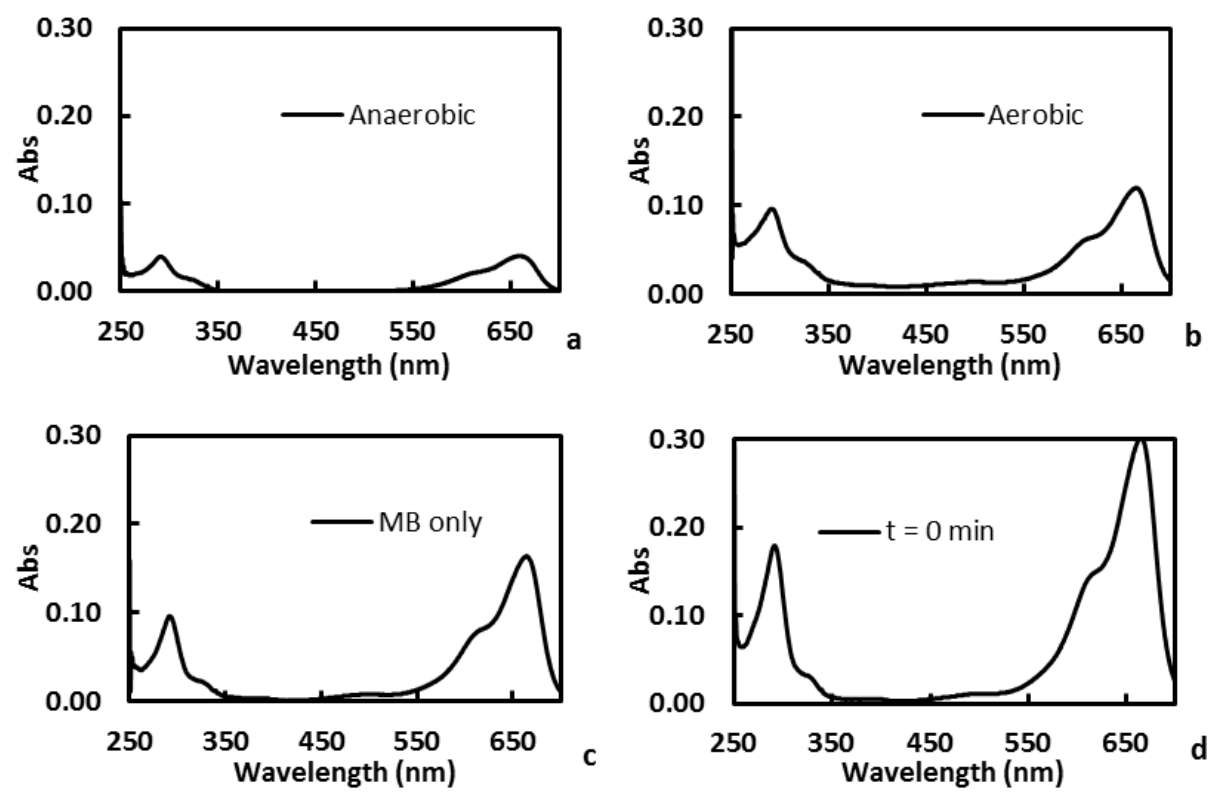

Figure 5. 5 Absorption spectra of the reaction solutions: a) absorption spectrum of the anaerobic reaction solution after 120 min treatment, b) absorption spectrum of the aerobic reaction solution after $\mathbf{1 2 0}$ min treatment, c) absorption spectrum of MB concentration after $120 \mathrm{~min}$ aerobic treatment, d) absorption spectrum of reaction solution before treatment. [DFN $]_{0}=1.0 \mathrm{mg} / \mathrm{L},[\mathrm{MB}]_{0}=1.5 \mathrm{mg} / \mathrm{L}, \mathrm{pH}$ unadjusted.

Under anaerobic conditions, only two of those degradation products were observed: the DP with $\mathrm{m} / \mathrm{z}$ of 202 and RT of $1.40 \mathrm{~min}$, and the DP with $\mathrm{m} / \mathrm{z}$ of 266 and RT of $5.14 \mathrm{~min}$. In addition, abundances of those two DP formed under anaerobic conditions were significantly lower than their abundances under aerobic conditions. The results confirm that diclofenac undergoes different degradation mechanisms when under aerobic conditions and anaerobic conditions. 

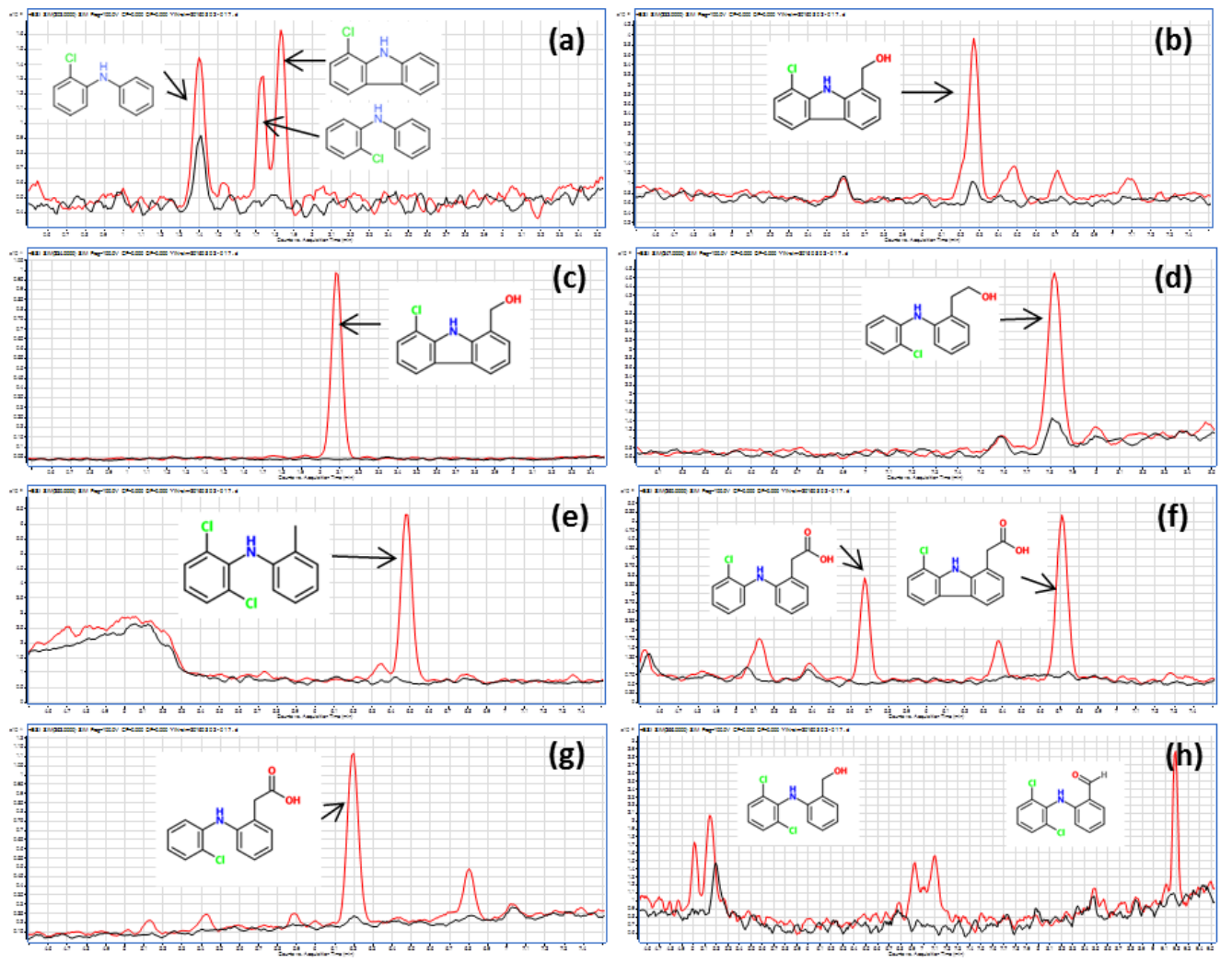

Figure 5. 6 Comparison of LC-MS/MS chromatograms of different diclofenac degradation products formation after $120 \mathrm{~min} \mathrm{MB/Red-light} \mathrm{treatment} \mathrm{under} \mathrm{aerobic} \mathrm{conditions}$ (red) and anaerobic conditions (black): (a)DPs with $\mathrm{m} / \mathrm{z}$ ratio of 202 ; (b) DPs with $\mathrm{m} / \mathrm{z}$ ratio of 232 ; (c) DPs with $\mathrm{m} / \mathrm{z}$ ratio of 234 ; (d) DPs with $\mathrm{m} / \mathrm{z}$ ratio of 247 ; (e) DPs with $\mathrm{m} / \mathrm{z}$ ratio of 250; (f) DPs with $\mathrm{m} / \mathrm{z}$ ratio of 260 ; (g) DPs with $\mathrm{m} / \mathrm{z}$ ratio of 262 ; (h) DPs with

$\mathrm{m} / \mathrm{z}$ ratio of 266 . X-axis stands for retention time, $y$-axis stands for signal intensity.

\subsubsection{Effect of background water constituents}

From the perspective of implementation, it is of importance to understand whether or not the background water constituents impose inhibitory effect on the efficiency of the proposed MB/Red-light photosensitized degradation processes, because vast literatures have reported that efficiency of ROS based degradation processes (e.g. $\mathrm{UV} / \mathrm{H}_{2} \mathrm{O}_{2}$, Fenton, Photo-Fenton, etc.) can be greatly hindered in the presence of background water constituents [96, 166, 243]. Therefore the effect of some typical background water constituents was studied in the present study, as a preliminary 
assessment of the potential application of $\mathrm{MB} /$ Red-light system in real wastewater. Experiments were carried out to examine the performance of $\mathrm{MB} /$ Red-light process in the presence of background water constituents, which are known to have great inhibitory effect on performance of existing AOPs like UV/TiO 2 , Fenton and Photo-Fenton, $\mathrm{UV} / \mathrm{H}_{2} \mathrm{O}_{2}[96,166]$. The experimental conditions were kept identical to that of Group A, three common water constituents were added separately to certain concentration level: $\mathrm{PO}_{4}{ }^{3-}(200 \mathrm{mg} / \mathrm{L}), \mathrm{HCO}_{3}{ }^{-}$ (200 mg/L), and NOM (10 mg/L).
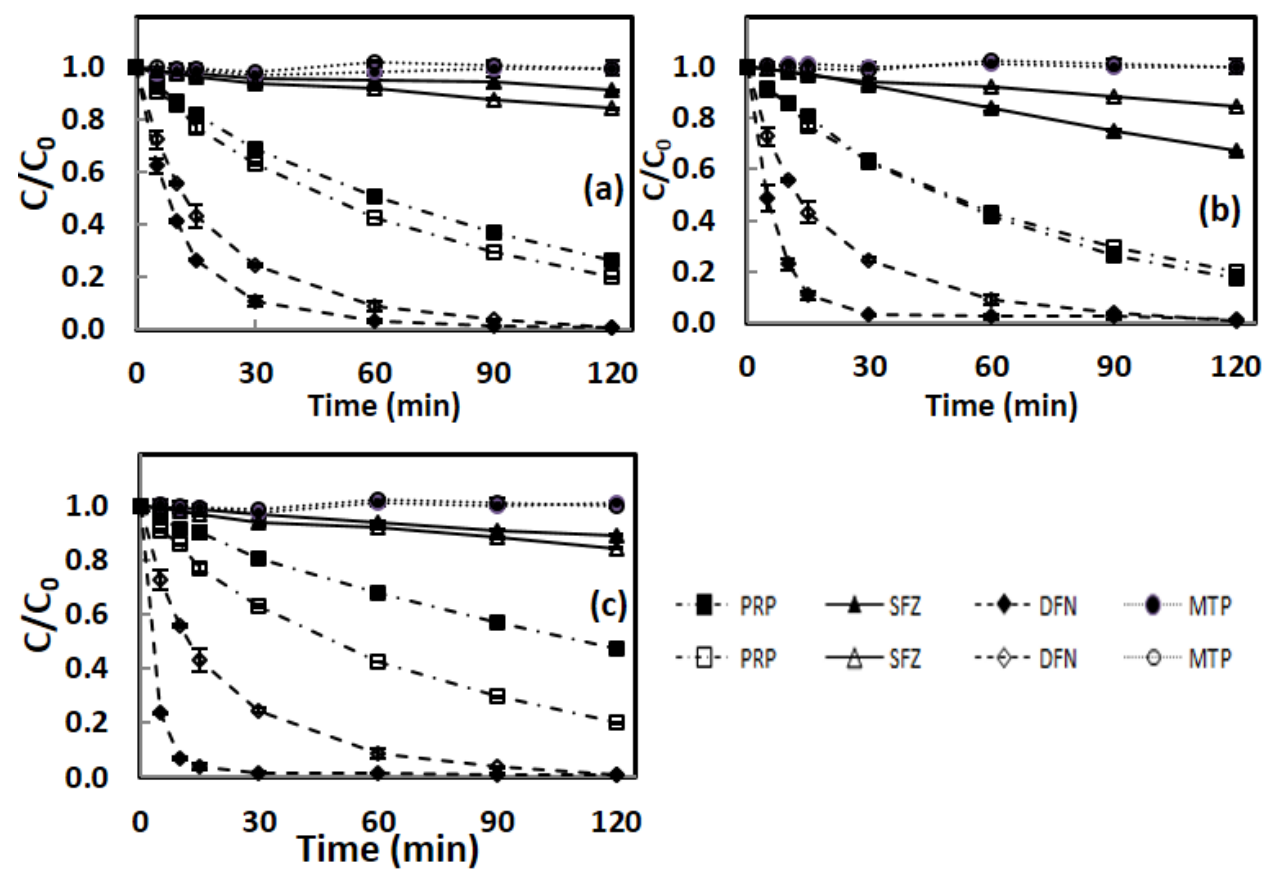

Figure 5. 7 Effect of the presence of: a) NOM $(10 \mathrm{mg} / \mathrm{L})$, b) bicarbonate ions $(200 \mathrm{mg} / \mathrm{L})$, and c) phosphate ions $(200 \mathrm{mg} / \mathrm{L})$ on the performance of $\mathrm{MB} / \mathrm{Red}$-light system.

$[D F N]_{0}=[M T P]_{0}=[P R P]_{0}=[S F Z]_{0}=1.0 \mathrm{mg} / \mathrm{L},[\mathrm{MB}]_{0}=1.5 \mathrm{mg} / \mathrm{L}, \mathrm{pH}$ was circum-neutral (6-7).

Background water constituents added (solid marks) or absent (open marks).

The results (Figure 5. 7, Table S5. 1) suggest that our proposed MB/Red-light process has potential to overcome inhibitory effect of background water constituents in complex water matrices. The results (Figure 5. 7a) show that the presence of up to $10 \mathrm{mg} / \mathrm{L}$ NOM does not pose significant adverse impact on the removal efficiency of all three 


\section{Chapter 5}

degradable model compounds. NOM can interfere with ROS based degradation processes by two major mechanisms: 1) scavenging of ROS and 2) competing for light irradiation (so called "inner filter effect"). NOM is a mixture of organic matters, so it is reactive towards many ROSs (e.g. hydroxyl radicals). NOM has strong light absorption in UV and near-UV range [177], so in some UV involved AOPs the presence of NOM usually lead to decrease in availability of UV light for ROS generation and therefore decrease in process performance. However, the aforementioned two factors are unlikely to take place in MB/Red-light processes. As documented in literature [177], only very small fraction of NOM contains electron-rich moieties. The dominant ROS in $\mathrm{MB} /$ Red-light system is ${ }^{1} \mathrm{O}_{2}$, which is very electrophilic and tend to react with electron-rich sites. Consequently, the scavenging of ROS by NOM in MB/Red-light system can be neglected. In addition, the "inner filter effect" caused by NOMs can be avoided in MB/Red-light process NOM has nearly no light absorption in the red light range (Figure S5. 8). Moreover, the presence of NOMs even slightly increased the degradation of DFN, which could be resulted from interaction among DFN, NOMs and MB. Further investigation is required to fully understand its mechanism.

Bicarbonate ions are most common water constituents and are the main constituents responsible for the alkalinity of a water body [243]. The results (Figure 5. 7b) show that $200 \mathrm{mg} / \mathrm{L}$ bicarbonate ions had no inhibitory effect on the degradation of model compounds. On the contrary, such inorganic ions impose severe inhibitory effect towards many existing AOPs, for instance S. Verma and M. Sillanpaa [243] that direct UV photolysis of anatoxin-a was greatly inhibited in the presence of $50 \mathrm{mg} / \mathrm{L}$ carbonate ions, and was totally inhibited with carbonate ions concentration higher than $100 \mathrm{mg} / \mathrm{L}$, by scavenging of radical species by carbonate ions. More interestingly, slight increase in pharmaceuticals degradation was observed with addition of $200 \mathrm{mg} / \mathrm{L}$ bicarbonate ions. This is due to the formation of carbonate radicals, which could be produced via several reactions including quenching of the triplet excited photosensitizer [247, 248]. Such radicals have relatively high oxidizing power $\left(\mathrm{E}^{0}=1.78 \mathrm{~V}\right.$ [247]), allowing rapid degradation of various organics in water. The generation of such radical species was a result of quenching of the triplet excited MB molecules by carbonate/bicarbonate ions, as described as bellow: 


$$
\begin{aligned}
& { }^{3} \mathrm{MB}+\mathrm{HCO}_{3}^{-} \rightarrow \mathrm{H}^{+}+\cdot \mathrm{MB}^{-}+\cdot \mathrm{CO}_{3}^{-} \\
& { }^{3} \mathrm{MB}+\mathrm{CO}_{3}^{2-} \rightarrow \bullet \mathrm{MB}^{-}+\cdot \mathrm{CO}_{3}^{-}
\end{aligned}
$$

The presence of $200 \mathrm{mg} / \mathrm{L} \mathrm{PO}_{4}{ }^{3-}$ imposes positive impact on DFN degradation and negative impact on degradation of PRP and SFZ (Figure 5. 7c). A study conducted by Knowles et al.[249] documented that phosphate ions can accelerate the protonation of the triplet $\mathrm{MB}$ and the protonated triplet $\mathrm{MB}$ has higher reactivity with electron donors, which leading to accelerated redox reactions between $\mathrm{MB}$ and electron donors. As aforementioned that DFN can undergo Type I photosensitization, i.e. redox reaction via electron transfer, it is rationale to attribute the promoted degradation of DFN to the accelerated Type I photosensitization pathway by the presence of phosphate ions. Our finding that high level phosphate ions has slight inhibitory effect on the degradation of PRP and SFZ is on the contrary to J. Brame et al.'s finding that phosphate ions have negligible adverse impact on singlet oxygen mediated degradation processes [177]. Considering the fact that scavenging of singlet oxygen by phosphate ions is unlikely to happen due to relatively low oxidizing power of singlet oxygen [177], and the fact that Type I photosensitization was promoted by the present of phosphate ions, the decrease in PRP and SFZ degradation is likely a result of reduced singlet oxygen formation caused by protonation of triplet MB [250].

\subsubsection{Effect of $\mathrm{pH}$}

Experiments were carried out to investigate the influence of solution $\mathrm{pH}$ on the total removal efficiency of three degradable pharmaceuticals in the MB/Red-light system (i.e. DFN, PRP, and SFZ), with pH of 4.6 (acidic condition), 7 (neutral condition), and 9 (alkaline condition). 

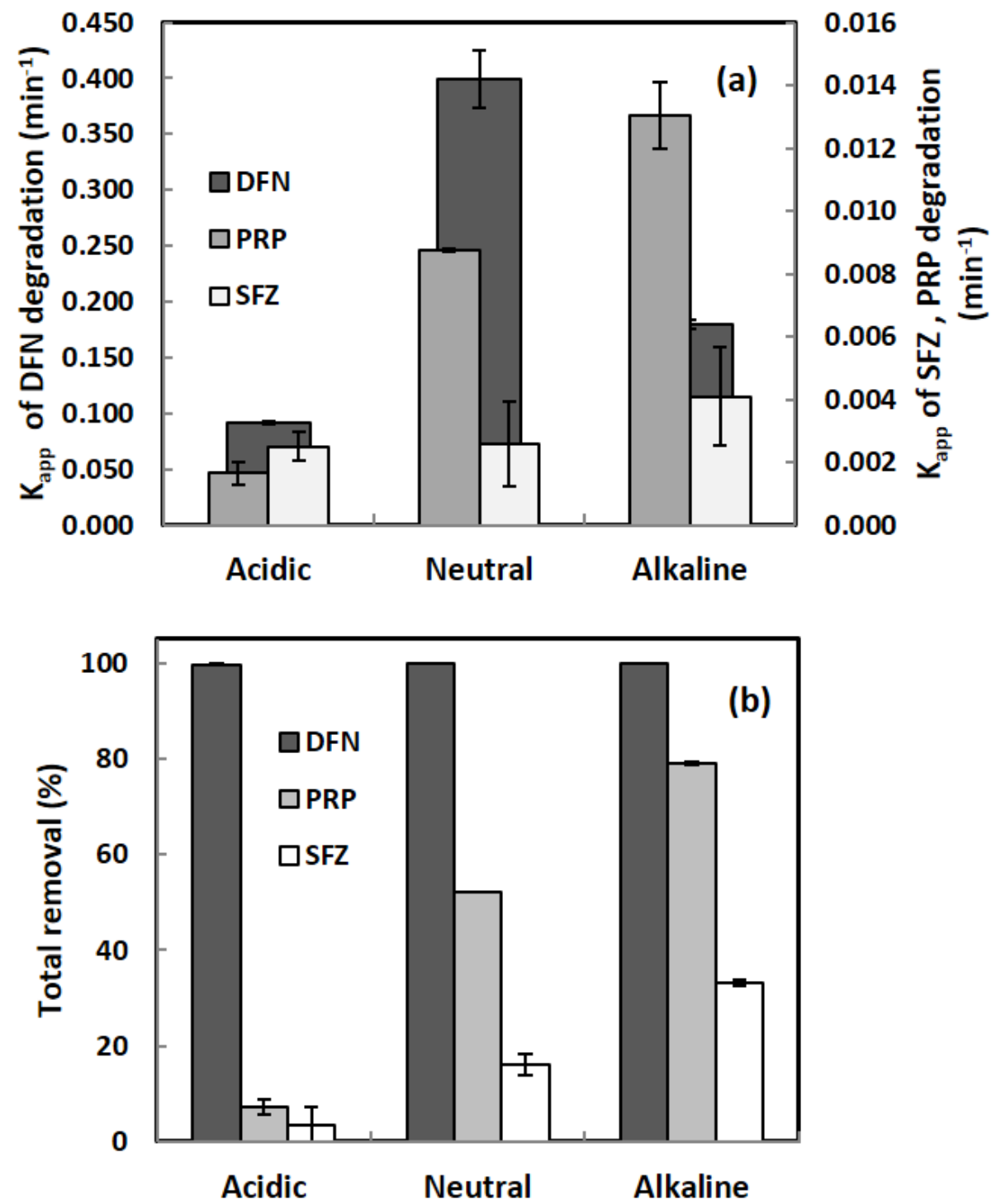

Figure 5. 8 Effect of pH on the MB/Red-light photosensitized pharmaceuticals degradation: a) kinetic constants of pharmaceuticals degradation, b) total removal of pharmaceuticals after $120 \mathrm{~min}$ treatment. $[\mathrm{DFN}]_{0}=[\mathrm{PRP}]_{0}=[\mathrm{SFZ}]_{0}=1.0 \mathrm{mg} / \mathrm{L},[\mathrm{MB}]_{0}=1.5$ $\mathrm{mg} / \mathrm{L}$, air equilibrated.

The results (Figure 5. 8) demonstrate that neutral and alkaline $\mathrm{pH}$ could promote $\mathrm{MB} /$ Red-light photosensitized PRP and SFZ degradation in terms of both initial degradation 
rate constants and total removal. Due to its high reactivity towards singlet oxygen and triplet MB, total removal of DFN reached near $100 \%$ even under acidic conditions. It should also be noticed that, neutral and alkaline $\mathrm{pH}$ is favourable for DFN degradation, regarding degradation rate. DFN experienced more rapid degradation under neutral and alkaline conditions. Moreover, the highest DFN degradation rate was obtained under neutral conditions, which can be attributed to accelerated direct reaction between MB and DFN (Type I photosensitization) under neutral conditions by the presence of phosphate ions as aforementioned in section 5.3.4. The positive effect of increased $\mathrm{pH}$ on the degradation of pharmaceuticals can be -as a likely explanation- partly attributed to deprotonation of the pharmaceutical compounds at higher $\mathrm{pH}$. At higher $\mathrm{pH}$, the model compounds were more present in deprotonated forms and therefore were more electron rich. Consequently, the attack by electrophilic oxidizing species (i.e. singlet oxygen) was facilitated. Other studies on singlet oxygen mediated micro-pollutant elimination processes have also reported the same finding [129]. Lee et al. [129] studied the photosensitized degradation of some emerging organic pollutants by using $\mathrm{C60} /$ silica under visible light irradiation. These authors investigated the influence of $\mathrm{pH}$ on degradation of some phenolic compounds, and found that although alkaline conditions can significantly favour the degradation of acetaminophen, bisphenol A, and 4-chlorophenol. Those authors attributed it to the increased deprotonation of those compounds and therefore increased reactivity towards electrophilic singlet oxygen. Another important factor should be taken into account is that $\mathrm{pH}$ exerts influence on MB transient states as well. A study conducted by Chen et al. [250] clearly pointed out that singlet oxygen production can be significantly promoted in basic $\mathrm{MB}$ solutions. Those authors found that the triplet $\mathrm{MB}$ molecules are more present in deprotonated form $\left({ }^{3} \mathrm{MB}^{+}\right)$under alkaline conditions which has higher quantum yield of singlet oxygen than that of protonated form triplet $\mathrm{MB}\left({ }^{3} \mathrm{MBH}^{2+}\right)$. Therefore, the promoted degradation of PRP and SFZ under alkaline conditions was resulted from two factors: 1) promoted deprotonation of the target pollutants and therefore promoted reactivity towards singlet oxygen; and 2) promoted singlet oxygen production under alkaline conditions. 


\section{Chapter 5}

\subsubsection{Red-LED as superior light source for the MB/Red-light system}

The red light LED used in this study has a light emission spectrum in the wavelength range of $600 \mathrm{~nm}$ to $700 \mathrm{~nm}$, which fit in the major light absorption area of MB (Figure S5. 5). The maximum light emission of the LED is around $660 \mathrm{~nm}$ which is coincident with the maximum light absorption of MB molecules, thereby ensuring good energy transfer from the electrical phase to the photosensitizer. When other light sources (such as incandescent, daylight or fluorescent) are used, much of the emitted spectrum (Figure S5. 9) does not coincide with the absorption peak of methylene blue, and will hence be left unused. Together with that, light energy increases with decreasing wavelength making this effect even stronger. As explained, LEDs transform electrical energy with relatively high efficiency into the photon domain at the right wavelength, making them the ideal light source for this application.

\subsection{Conclusions}

This study shows the proof of principle of using red light LED as light source and $\mathrm{MB}$ as photosensitizer for pharmaceuticals removal from water. Three of the four tested model compounds were degraded under the applied conditions. The degradation rates varied among the compounds. MB/Red-light degradation of DFN and PRP exhibited a rapid degradation (0.0572 $\mathrm{min}^{-1}$ and $0.0170 \mathrm{~min}^{-1}$, respectively) with total removal of $98.94 \%$ and $79.98 \%$ after 120 min treatment, respectively, but the degradation of SFZ was much slower $\left(k_{\text {app }}=0.0022 \mathrm{~min}^{-1}\right)$. Singlet oxygen plays a crucial role for PRP and SFZ degradation. For DFN degradation, Type I photosensitization involving direct reaction with MB was also of importance, in addition to Type II photosensitization involving DO. Three common background water constituents have no significant adverse impact on the process performance, suggesting that the proposed $\mathrm{MB} /$ Red-light system has great potential to overcome inhibitory effects of background water constituents in complex water matrices often encountered in waste water and waste water effluents. This is advantageous over many other existing AOP technologies. Effect of $\mathrm{pH}$ on the efficacy of $\mathrm{MB} /$ Red-light system has been studied, and the results suggest that higher $\mathrm{pH}$ exerts significant positive impact. Last but not least, the red light LED used in the present study was found to be the optimum light source for the proposed MB/Red-light system, because the light emitting spectrum of 
the LED fits well with the light absorption of $\mathrm{MB}$ in the red light range. For further research, more advanced measurement should be performed to confirm structures of the DPs of target pollutants. Systematic and comprehensive toxicity tests should be performed to examine efficacy of the proposed $\mathrm{MB} /$ Red-light process in reducing eco-toxicity risk of pharmaceutical containing wastewater. From practical point of view, effect of operational parameters should also be studied in future work to provide better understanding for process optimization.

\subsection{Acknowledgements}

This work was performed in the cooperation framework of Wetsus, European Centre of Excellence for Sustainable Water Technology (www.wetsus.eu). Wetsus is cofunded by the Dutch Ministry of Economic Affairs and Ministry of Infrastructure and Environment, the Province of Fryslân, and the Northern Netherlands Provinces. The authors would like to thank the participants of the research theme "Advanced water treatment" for the fruitful discussions and their financial support. The authors gratefully acknowledge Ton van der Zande for the realization of the instrumental analysis, and Sofia SemitsoglouTsiapou, Gerwin Steen, Cong Bao and Yang Lei for the fruitful discussion. 
Chapter 5

\section{Supplementary information}

(1)

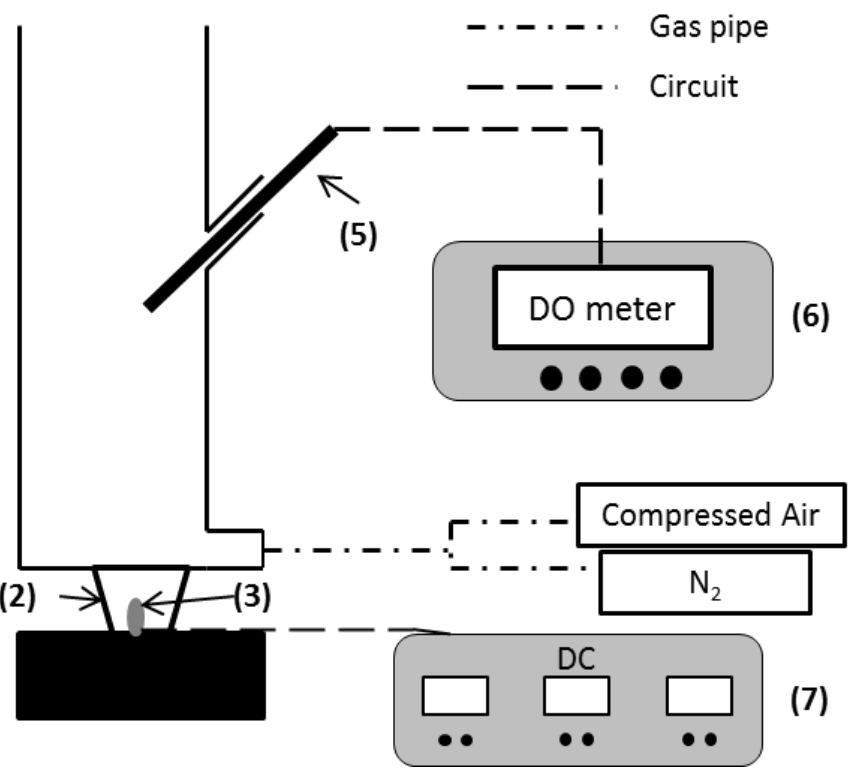

Figure S5. 1 Schematic of the set-up; (1) glass cylinder reactor, (2) lens, (3) red light LED, (4) cooling plate, (5) dissolved oxygen sensor, (6) dissolved oxygen meter, (7) DC power supply.

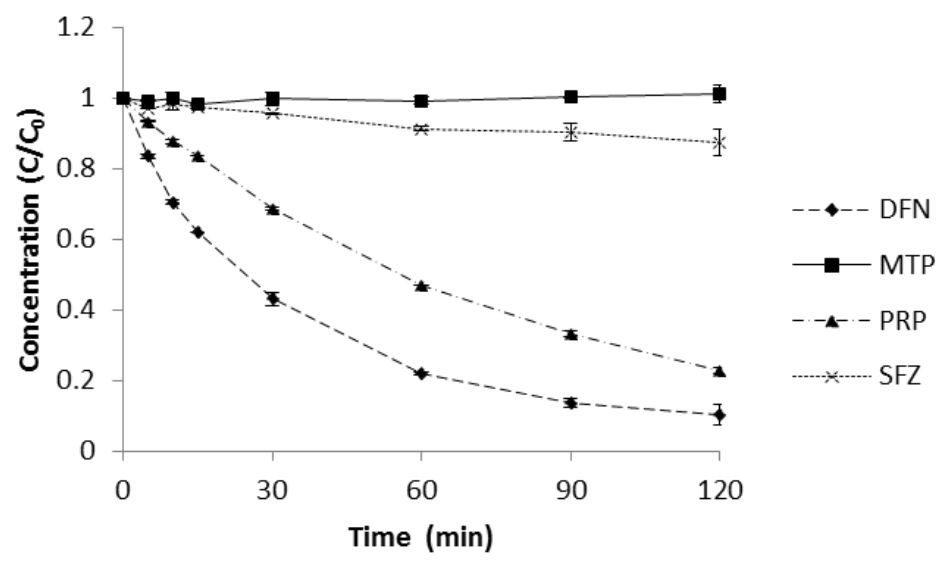

Figure S5. $2 \mathrm{MB} /$ red-light photosensitized degradation of individual model compound as a function of time. $[D F N]_{0}=[M T P]_{0}=[P R P]_{0}=[S F Z]_{0}=1.0 \mathrm{mg} / \mathrm{L},[\mathrm{MB}]_{0}=1.5 \mathrm{mg} / \mathrm{L}, \mathrm{pH}$ unadjusted. 
Chapter 5

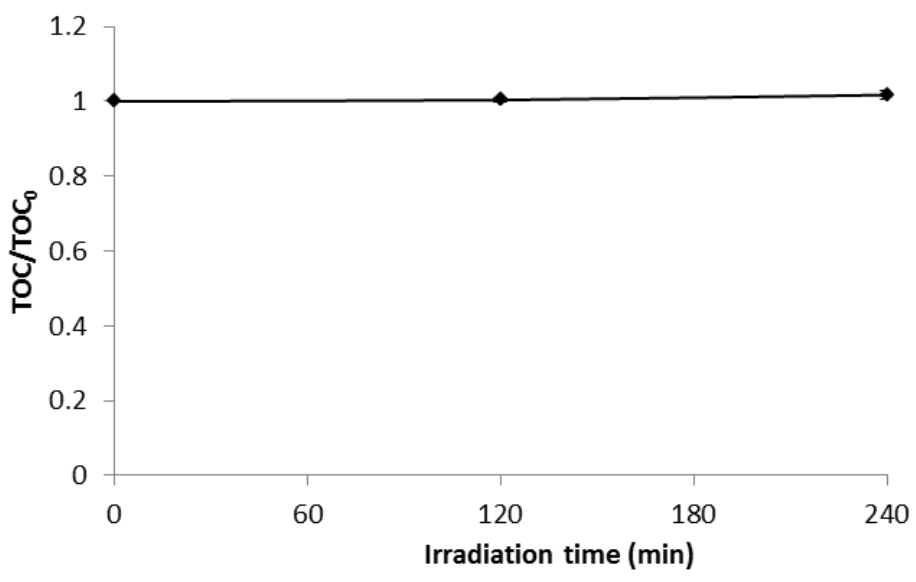

Figure S5. 3 TOC level profile as a function of time during MB/red-light photosensitized degradation of pharmaceuticals. $[\mathrm{DFN}]_{0}=[\mathrm{MTP}]_{0}=[\mathrm{PRP}]_{0}=[\mathrm{SFZ}]_{0}=1.0 \mathrm{mg} / \mathrm{L},[\mathrm{MB}]_{0}=1.5$ $\mathrm{mg} / \mathrm{L}, \mathrm{pH}$ unadjusted.

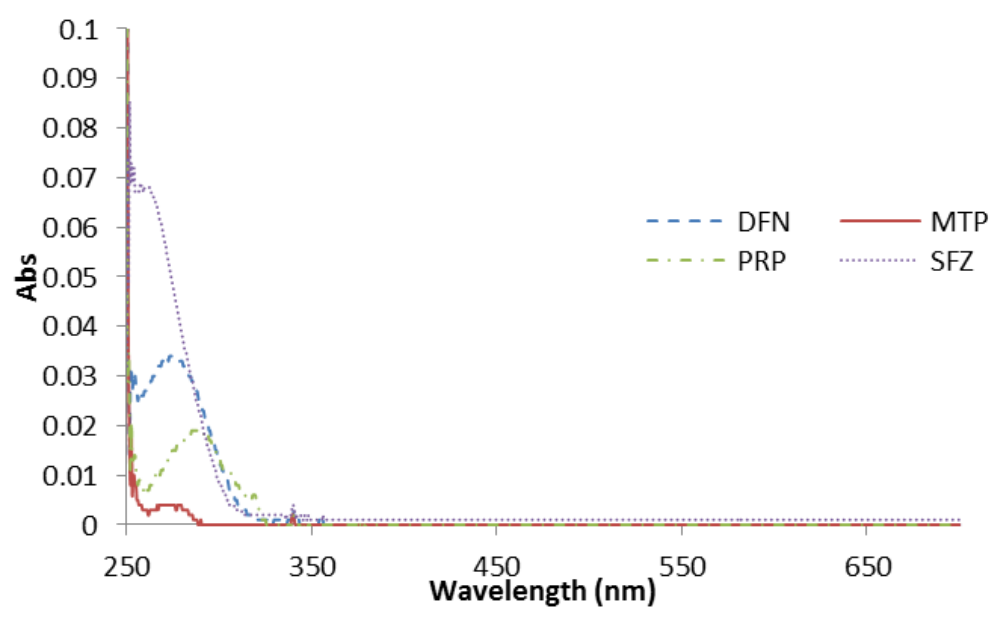

Figure S5. 4 Absorption spectra of model compounds aqueous solutions (250nm 700nm) 
Chapter 5

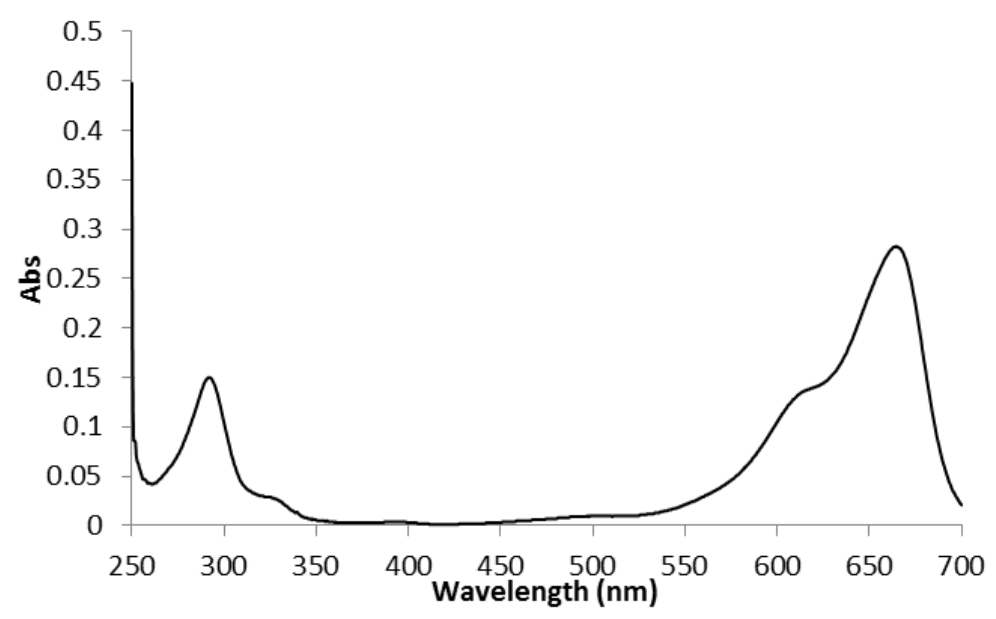

Figure S5. 5 Absorption spectrum of MB aqueous solution (250nm - 700nm)

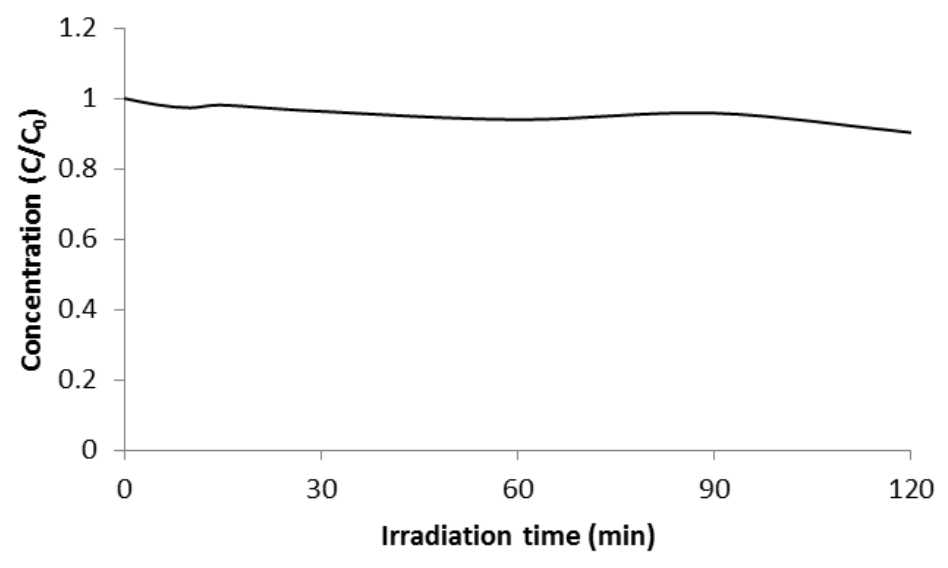

Figure S5. $6 \mathrm{MB}$ concentration profile as a function of time, without presence of any pharmaceutical compound, under red light irradiation and anaerobic conditions 
Chapter 5

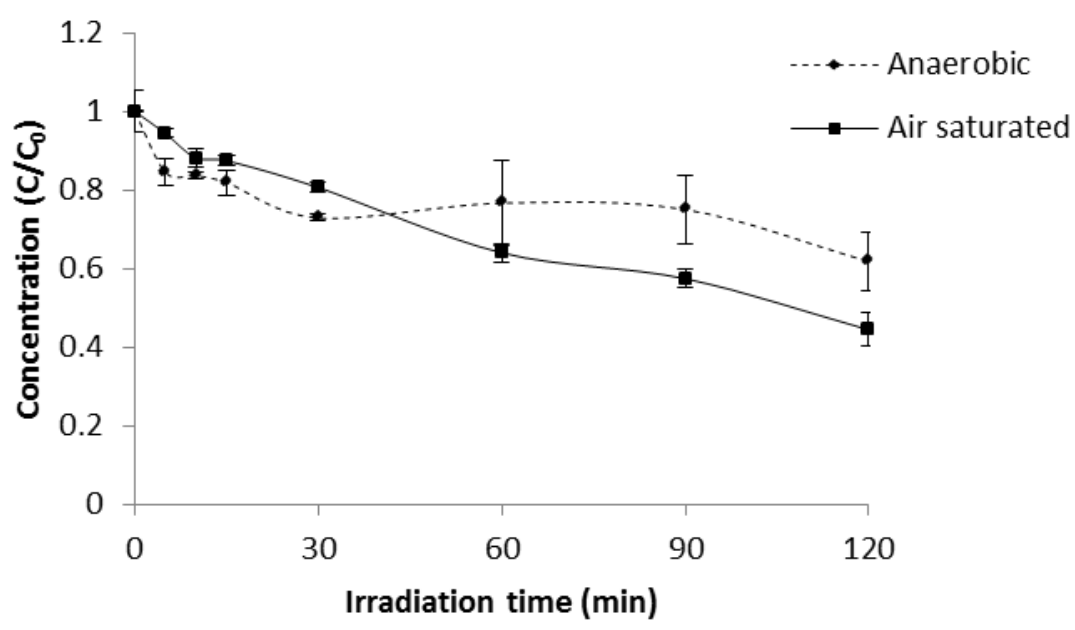

Figure S5. 7 MB concentration as a function of time, under aerobic (Group A) and anaerobic conditions (Group D) 


\section{Chapter 5}

Table S5. 1 Time-based pseudo-first-order kinetic constants and removal of pharmaceuticals after $120 \mathrm{~min} \mathrm{MB/Red-light} \mathrm{treatment} \mathrm{in} \mathrm{presence} \mathrm{of} \mathrm{different}$ background water constituents

\begin{tabular}{|c|c|c|c|c|}
\hline Matrix & Compound & $k_{\text {app }}\left(\min ^{-1}\right)$ & $\mathbf{R}^{2}$ & Total removal (\%) \\
\hline \multirow[t]{4}{*}{$10 \mathrm{mg} / \mathrm{L} \mathrm{NOM}$} & Diclofenac & 0.089 & 0.999 & 98.97 \\
\hline & Propranolol & 0.013 & 0.997 & 73.40 \\
\hline & Sulfamethoxazole & 0.002 & 0.359 & 8.72 \\
\hline & Metoprolol & 0.001 & 0.314 & 0.25 \\
\hline \multirow{4}{*}{$\begin{array}{c}200 \mathrm{mg} / \mathrm{L} \\
\text { carbonate/bicarbonate } \\
\text { ions }\end{array}$} & Diclofenac & 0.148 & 1.000 & 98.86 \\
\hline & Propranolol & 0.015 & 0.987 & 82.93 \\
\hline & Sulfamethoxazole & 0.002 & 0.999 & 32.97 \\
\hline & Metoprolol & -0.001 & 0.966 & 0.04 \\
\hline \multirow[t]{4}{*}{$200 \mathrm{mg} / \mathrm{L}$ phosphate ions } & Diclofenac & 0.236 & 0.960 & 98.96 \\
\hline & Propranolol & 0.008 & 0.933 & 52.54 \\
\hline & Sulfamethoxazole & 0.001 & 0.944 & 10.86 \\
\hline & Metoprolol & 0.001 & 0.748 & 0.71 \\
\hline
\end{tabular}


Chapter 5

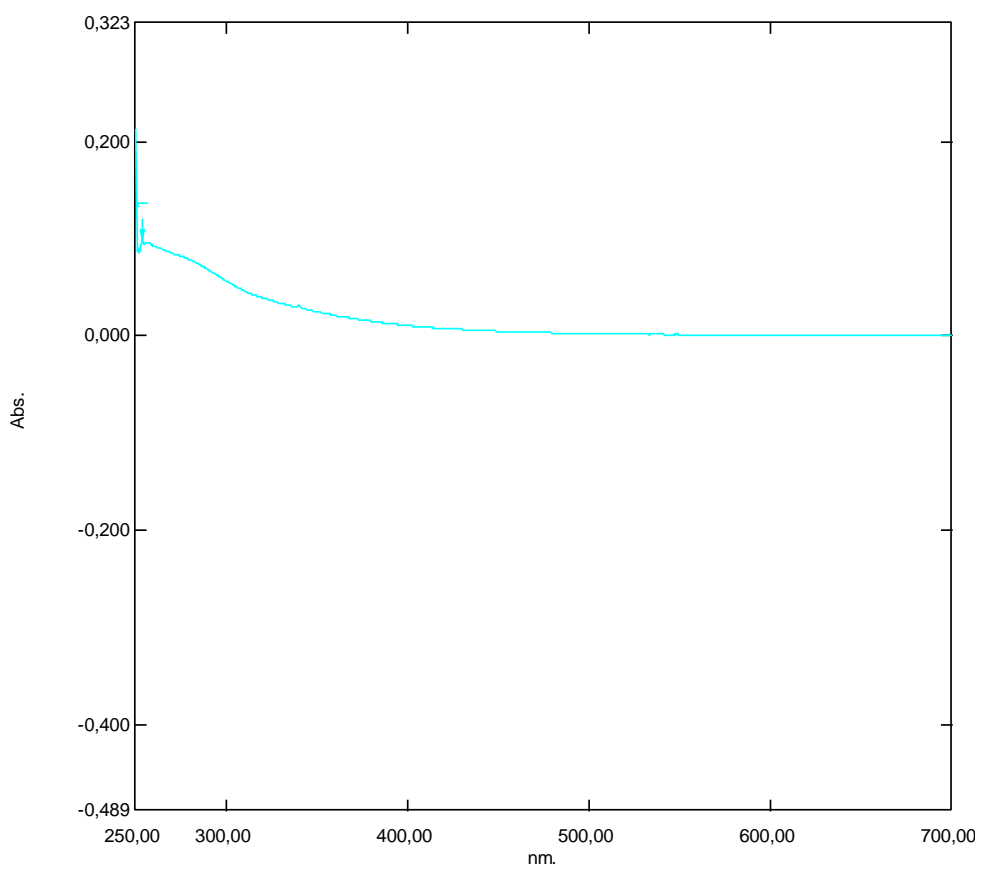

Figure S5. 8 Absorption spectrum of NOM aqueous solution (250nm - 700nm)
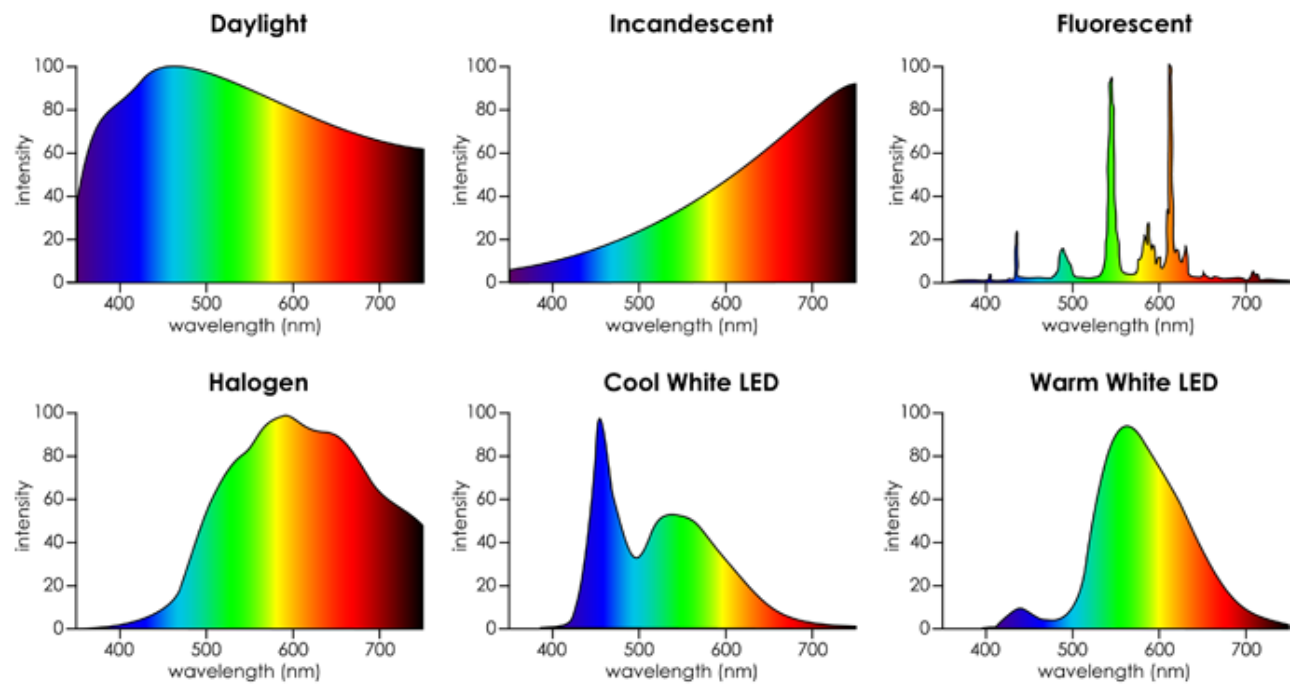

Figure S5. 9 Light emission spectra of typical light sources (Source:

http://www.lightingschool.eu/portfolio/understanding-the-light// 


\section{Chapter 6}

\section{Operational parameters affecting MB/Red-}

\section{light photosensitized degradation of}

\section{pharmaceuticals}

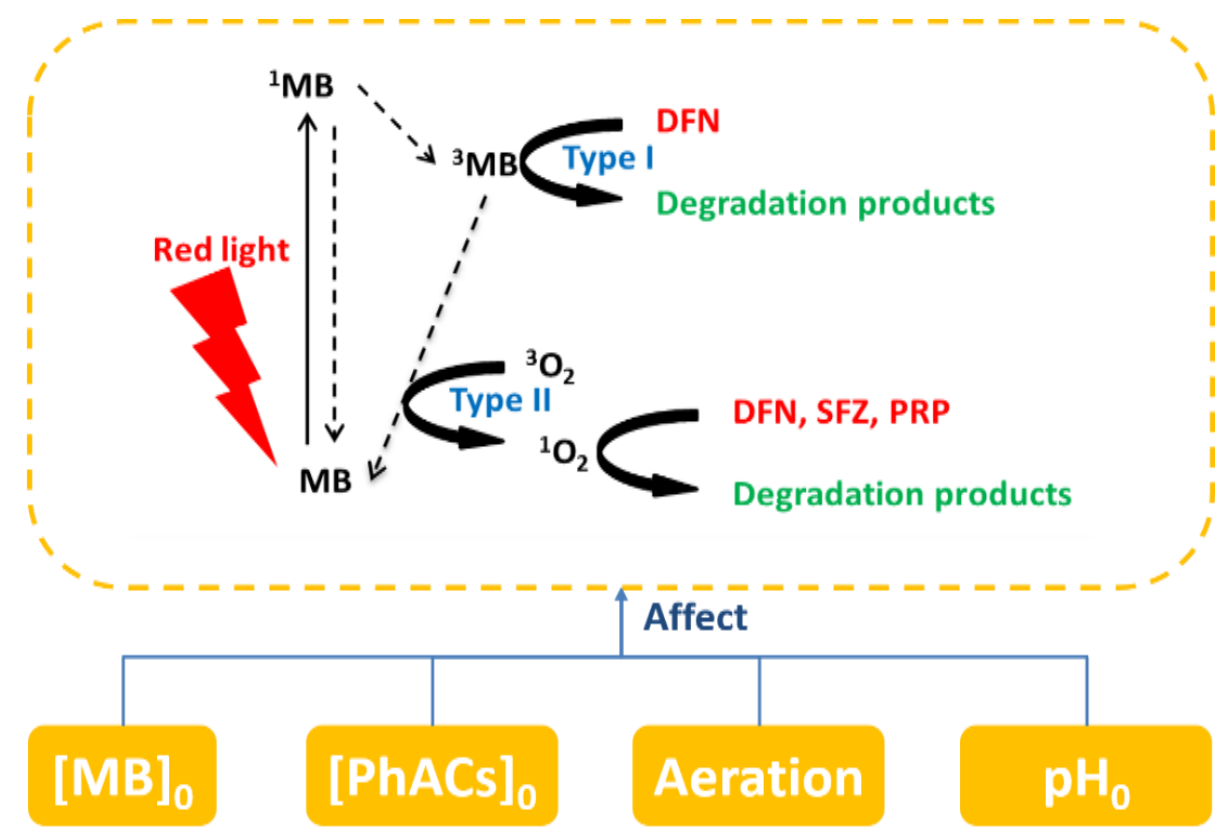

This Chapter has been published as:

Y. Ye, Y. Luo, H. Bruning, D. Yntema, H. Rijnaarts, Operational parameters affecting MB/Redlight photosensitized degradation of pharmaceuticals, Journal of Photochemistry \& Photobiology, A: Chemistry 348 (2017) pp. 96-101 


\section{Chapter 6}

\section{Abstract}

The methylene blue photosensitization under red light irradiation ( $\mathrm{MB} /$ Red-light) is a promising and powerful tool for removal of pharmaceuticals from wastewater. To further develop this new technology, the present work aimed at studying the effect of operational parameters on the performance of $\mathrm{MB} /$ Red-light pharmaceuticals removal processes. Three pharmaceuticals, i.e. diclofenac (DFN), propranolol (PRP), and sulfamethoxazole (SFZ), were used as model compounds, and degradation rate constants and total compound removal were examined. The three operational parameters studied were initial $M B$ concentration $(0-5.0 \mathrm{mg} / \mathrm{L})$, initial pharmaceutical concentration $(0.1-2.0 \mathrm{mg} / \mathrm{L})$, aeration rate $(0-5.0 \mathrm{~L} / \mathrm{min})$, and for DFN also the $\mathrm{pH}$. The results show that degradation of pharmaceuticals was promoted with increasing initial MB concentration at values of [MB] below $0.5 \mathrm{mg} / \mathrm{l}$, and leveled off to constant values at [MB] values higher than $2.0 \mathrm{mg} / \mathrm{l}$. Initial pharmaceutical concentration and aeration rate were found to have no significant impact. Moreover, rapid degradation of pharmaceuticals can take place even at low initial dissolved oxygen concentrations $(2.0 \mathrm{mg} / \mathrm{L}$, i.e. situations without aeration). In order to better understand the effect of $\mathrm{pH}$ on the $\mathrm{MB} /$ Red-light pharmaceutical degradation processes, DFN was chosen for more detailed investigation, with identification of the degradation products formed under neutral and alkaline conditions identified by LC$\mathrm{MS} / \mathrm{MS}$. The $\mathrm{pH}$ was found to play an important role on the transformation pathways and formation of degradation products. 


\subsection{Introduction}

In recent decades, pharmaceuticals have been found present at various concentration levels in the aqueous environment $[7,14,27]$. As reported by Klavarioti et al. [190], pharmaceuticals were detected in not only ground and surface water but also drinking water according to a broad range of studies [5-11]. Due to lack of effective regulation on acceptable emissions of the pharmaceutical compounds to natural water systems, as well as growing evidence of the associated potential hazardous impacts on the environment and human health, concerns are growing rapidly. Studies have indicated that the presence of pharmaceuticals in the environment imposes hazards to aquatic life at different levels, from algae to fish, even at low concentrations [27]. The presence of pharmaceuticals in surface, ground, and drinking water reveals the low efficacy of conventional wastewater treatment processes and drinking water production processes, e.g. biological processes, sedimentation, filtration, coagulation/flocculation, in removing the micro-pollutants $[64,190]$. Therefore, to cope with the problem associated with pharmaceuticals in water, more advanced treatment processes are required.

In our recent work [65], we addressed the feasibility of using red-light induced methylene blue photosensitization (MB/Red-light) to degrade diclofenac (DFN), sulfamethoxazole (SFZ) and propranolol (PRP) in aqueous solutions, where the primary oxidizing species is singlet oxygen $\left({ }^{1} \mathrm{O}_{2}\right)$. The $\mathrm{MB} /$ Red-light photosensitization resulted in rapid elimination of DFN and PRP within 2 hours, with total removal of $99.7 \%$ and $83.4 \%$ respectively; the degradation of SFZ under identical conditions was much slower with lower total removal (21.8\%). The MB/Red-light photosensitized degradation of pharmaceuticals has several advantages: (1) unlike the solid photosensitizers, MB is well dissolved in water, therefore the photosensitized produced ${ }^{1} \mathrm{O}_{2}$ has a better chance to react with pharmaceuticals in water phase; (2) the methylene blue is degraded during the process; (3) the "inner filter effect", which means absorption of light by natural organic matters (NOMs), is less because light absorbance by NOMs in the red light range is much less than that in the UV and near UV range [177].

For realizing the implementation of the $\mathrm{MB} /$ Red-light process, there is a need to optimize the $\mathrm{MB} /$ Red-light process to judge the feasibility and facilitate its real life 


\section{Chapter 6}

applications. Although in our recent study, the authors reported that neutral and alkaline $\mathrm{pH}$ could favor the MB/Red-light pharmaceuticals removal processes [65]. However, the effect of other operational parameters on the MB/Red-light pharmaceutical compounds degradation process has not been studied yet. Therefore, the objective of the present work was to investigate the effect of various operational parameters, i.e. initial MB concentration, initial pharmaceutical concentration, and aeration rate on the $\mathrm{MB} /$ Red-light pharmaceutical removal process, in terms of degradation rate constants and total removal. Moreover, in order to better understand the effect of $\mathrm{pH}$ on the $\mathrm{MB} /$ Red-light pharmaceutical degradation processes, DFN was chosen for more detailed investigation, with identification of the degradation products formed under neutral and alkaline conditions identified by LCMS/MS.

\subsection{Materials and Methods}

\subsubsection{Materials}

Diclofenac (DFN), sulfamethoxazole (SFZ), metoprolol (MTP), and propranolol (PRP) were purchased from Sigma-Aldrich (Germany) and were used as received. Stock solutions of each pharmaceutical compound were prepared at concentrations of $200 \mathrm{mg} / \mathrm{L}$ for each compound. The photosensitizer methylene blue was purchased from Boom BV (The Netherlands), and a MB stock solution at $200 \mathrm{mg} / \mathrm{L}$ was prepared. Sodium dihydrogen phosphate and di-sodium hydrogen phosphate dehydrate $(\geq 99.5 \%$, purchased from Boom BV, The Netherlands) were used to prepare the buffer solutions. All stock solutions and reaction solutions were prepared using ultrapure water from a Milli-Q Advantage A10 system (Merck Millipore, Darmstadt, Germany).

\subsubsection{Experimental procedures}

Photosensitized experiments were conducted in a set-up which has been described in details elsewhere [65]. In each run, $500 \mathrm{~mL}$ reaction solutions were prepared with designated initial MB concentration, initial pharmaceutical concentration, and $\mathrm{pH}$. For the experiments studying the effect of $\mathrm{pH}, 50 \mathrm{mM}$ phosphate buffer was used to maintain the designated $\mathrm{pH}$ value, while all other experiments were conducted in natural $\mathrm{pH}$ (circumneutral) and no buffer was used. Aeration rate was set to designated values. The red-LED 
(type LZ4-00R200 from LED-ENGIN) was mounted onto a cooling plate under the bottom of the reactor. The red light LED has an emission spectral between $600 \mathrm{~nm}$ to $700 \mathrm{~nm}$ with a maximum emission at $660 \mathrm{~nm}$. The red-light LED was placed under the bottom of the reactor. The radiant power density entering the reactor was measured to be $1.77 \mathrm{~W}$, by using a THORLABS S120C photodiode power sensor (THORLABS, USA). Under the applied irradiation conditions, the photon flux was calculated to be around $9.77 \mu \mathrm{mol} / \mathrm{s}$. During the experiments, samples were taken at designated times and the total irradiation time of each run was 120 minutes. All experiments were carried out in ultrapure water (MilliPore MilliQ), and in duplicates.

\subsubsection{Sample analysis}

The MB concentration was measured with a UV/Vis spectrophotometer (Shimadzu UV-1800) at $664.5 \mathrm{~nm}$. Pharmaceutical concentrations were analyzed using an Agilent LCMS/MS system consisting of Agilent infinity 1260 LC-system (degasser, binary pump, auto sampler with cooled tray and column oven) and Agilent 6420 triple Quadrupole Mass Spectrometer with Electrospray ion source. The compounds were separated using a Phenomenex Gemini Phenyl-Hexyl column (150x3 mm, particle size $5 \mu \mathrm{m})$ and a gradient of acetonitrile ( 5 to $90 \%$ ) and AmmoniumFormate buffer in water. The compounds were detected and quantified on the 6420-QQQ-MS using compound specific multiple Dynamic MRM transitions. Detailed information of the analytical method used can be found in literature [220].

\subsection{Results and Discussion}

\subsubsection{Effect of initial MB concentration}

To investigate the influence of the initial MB concentration on the degradation rate and total removal of pharmaceuticals, experiments were carried out with varied initial MB concentrations. The initial pharmaceuticals concentrations were kept at around $1.0 \mathrm{mg} / \mathrm{L}$, the $\mathrm{pH}$ was unadjusted, and the experiments were carried out under air equilibrated conditions.

To avoid the interference of produced transformation products on the kinetics, data of the first 15 minutes were used to calculate the pseudo-first-order apparent rate 


\section{Chapter 6}

constants ( $k_{a p p}$ ) according to the equation described in literature [90]. Moreover, considering the fact that the formation of intermediates may influence the degradation rate, total removal values of pharmaceuticals after 120 minutes was calculated as an indication of efficacy within the time frame of treatment in the tested systems. The results are shown in Figure 6. 1a. In general, a certain initial MB concentration was needed to initiate the degradation of pharmaceuticals. With initial MB concentration lower than $0.01 \mathrm{mg} / \mathrm{L}$, none of the tested pharmaceuticals had noticeable degradation. With initial MB concentration of $0.1 \mathrm{mg} / \mathrm{L}$, degradation of DFN and PRP was obtained but at rather low degradation rate, while no degradation of SFZ was observed. By increasing initial MB concentration from 0.1 $\mathrm{mg} / \mathrm{L}$ to $0.5 \mathrm{mg} / \mathrm{L}$, a significant increase in pharmaceuticals degradation was obtained. SFZ experienced slow degradation when initial MB concentration was higher than $0.5 \mathrm{mg} / \mathrm{L}$, and the degradation of SFZ did not change significantly, when the initial MB concentration changed from $0.5 \mathrm{mg} / \mathrm{L}$ to $5.0 \mathrm{mg} / \mathrm{L}$. PRP degradation took place when initial $\mathrm{MB}$ concentration was higher than $0.1 \mathrm{mg} / \mathrm{L}$. With increased initial MB concentration till 1.0 $\mathrm{mg} / \mathrm{L}$, PRP degradation experienced a significant increase. However, with further increased initial $\mathrm{MB}$ concentration, from $1.0 \mathrm{mg} / \mathrm{L}$ to $5.0 \mathrm{mg} / \mathrm{L}$, no significant increase in PRP degradation rate constant was obtained. The DFN degradation was obtained when initial MB concentration was higher than $0.1 \mathrm{mg} / \mathrm{L}$. The DFN did not degrade significantly at initial $\mathrm{MB}$ concentrations lower than $0.1 \mathrm{mg} / \mathrm{L}$, and degraded rapidly when initial $\mathrm{MB}$ concentration was higher than $1 \mathrm{mg} / \mathrm{L}$. DFN degradation rate constant increased significantly with increased initial MB concentration till $1.5 \mathrm{mg} / \mathrm{L}$. With further increased initial MB concentration from $1.5 \mathrm{mg} / \mathrm{L}$ to $5.0 \mathrm{mg} / \mathrm{L}$, the DFN degradation rate constant was nearly constant.

For the pharmaceuticals total removal after 120 minutes treatment, initial MB concentration also influenced, as shown in Figure 6. 1b. In general, the total removal of pharmaceuticals increased by increasing initial MB concentration at low range. Like the degradation kinetics, the influence of initial MB concentration on the total removal is also compound-specific. The total removal of SFZ was negligible when initial MB concentration was lower than $0.1 \mathrm{mg} / \mathrm{L}$. With increased initial MB concentration from $0.1 \mathrm{mg} / \mathrm{L}$ to $0.5 \mathrm{mg} / \mathrm{L}$, the total removal of SFZ was significantly increased; while further increased initial MB concentration higher than $0.5 \mathrm{mg} / \mathrm{L}$ did not cause significant change in SFZ total removal. 
For PRP, no removal was obtained with initial MB concentration lower than $0.1 \mathrm{mg} / \mathrm{L}$. By increasing the initial MB concentration from $0.01 \mathrm{mg} / \mathrm{L}$ to $1.0 \mathrm{mg} / \mathrm{L}$, the total removal of PRP was drastically increased to 78.1\%; however, with further increased initial MB concentration from $1.0 \mathrm{mg} / \mathrm{L}$ to $5.0 \mathrm{mg} / \mathrm{L}$, the change in PRP total removal became negligible. DFN had no removal when initial MB concentration was lower than $0.1 \mathrm{mg} / \mathrm{L}$. DFN removal efficiency experienced a sharp increase to $81.1 \%$, by increasing the initial MB concentration till $0.5 \mathrm{mg} / \mathrm{L}$. With further increased initial MB concentration, from $0.5 \mathrm{mg} / \mathrm{L}$ to $1.5 \mathrm{mg} / \mathrm{L}$, the DFN removal efficiency still slightly increased (from $81.1 \%$ to $98.3 \%$ ), and no significant change was obtained when Initial MB concentration was increased from $1.5 \mathrm{mg} / \mathrm{L}$ to 5.0 $\mathrm{mg} / \mathrm{L}$.

To conclude, as demonstrated by the results, degradation of all model compounds, in terms of both degradation rate and total removal, were increased by increasing initial MB concentration in relatively low initial MB concentration level, and became independent of initial $M B$ concentration with further increased initial $M B$ concentration when the optimal initial MB concentration of $1.5 \mathrm{mg} / \mathrm{L}$ was reached. A similar phenomenon has been well documented by A. Achilleos et al. [107], who investigated the addition of $\mathrm{H}_{2} \mathrm{O}_{2}$ in UV-A/TiO photo-catalytic degradation of DFN. They found that an optimal H2O2 concentration exists above which further increased $\mathrm{H}_{2} \mathrm{O}_{2}$ concentration would no longer promote the degradation process. Those authors attributed this to the fact that $\mathrm{H}_{2} \mathrm{O}_{2}$ act as both radical production enhancer and radical scavenger, and the scavenging effect of $\mathrm{H}_{2} \mathrm{O}_{2}$ increased dramatically with increased $\mathrm{H}_{2} \mathrm{O}_{2}$ concentration. Other researchers have reported the similar phenomenon when applying MB as photosensitizer for disinfection and solar water treatment $[187,188,251]$, and they explained it in terms of hindrance in light penetration and self-aggregation of $\mathrm{MB}$ by elevated $\mathrm{MB}$ concentration. In this study, the consumption of $\mathrm{MB}$ did not increase with further increased initial $\mathrm{MB}$ concentration (Figure 6. 1c); indicating that scavenging effect of $\mathrm{MB}$ had no increase with initial $\mathrm{MB}$ concentration higher than $1.5 \mathrm{mg} / \mathrm{L}$. Therefore, the hindrance in light penetration and self-aggregation of $\mathrm{MB}$ may play an important role when $\mathrm{MB}$ concentration exceeds the optimal range. 
Chapter 6
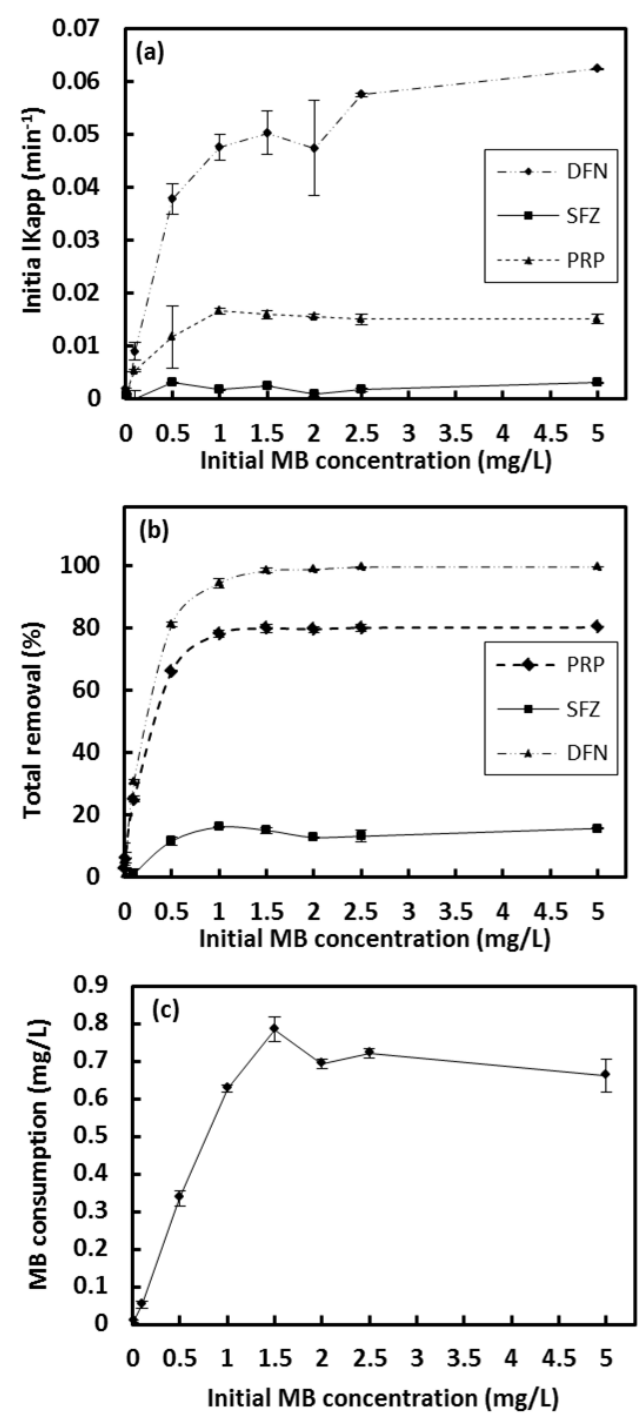

Figure 6. 1 Effect of initial MB concentration on the initial degradation rate constants of pharmaceuticals (a), total removal after 120 minutes (b), and MB consumption (c). Initial pharmaceutical concentration $=1.0 \mathrm{mg} / \mathrm{L}, \mathrm{pH}$ unadjusted, air equilibrated.

\subsubsection{Effect of initial pharmaceutical concentration}

To investigate the influence of initial pharmaceutical concentration on the photosensitized degradation of pharmaceuticals, in terms of degradation rate and total removal, experiments were carried out with varied initial pharmaceutical concentrations. 
The optimal initial MB concentration of $1.5 \mathrm{mg} / \mathrm{L}$ (see section 6.3.3.1), was applied, the $\mathrm{pH}$ was unadjusted, and the experiments were carried out in air equilibrated condition.

The obtained results are shown in Figure 6. 2. The results indicate, unlike the Initial $\mathrm{MB}$ concentration, that the varied initial pharmaceutical concentration had no significant influence on pharmaceutical degradation. By decreasing the initial pharmaceutical concentration by a factor of 20 , from $2.0 \mathrm{mg} / \mathrm{L}$ to $0.1 \mathrm{mg} / \mathrm{L}$, the degradation rate constant Kapp had no significant change (see Figure 6. 2a). Regarding the total removal, initial pharmaceutical concentration had no significant effect as well, as shown in Figure 6. 2b. With decreased initial pharmaceutical concentrations from $2.0 \mathrm{mg} / \mathrm{L}$ to $0.1 \mathrm{mg} / \mathrm{L}$, the total removal of model compounds were nearly constant. This finding can be attributed to the following facts: (1) the availability of reactive species was constant under the same applied irradiation conditions and MB loading; (2) when initial pharmaceuticals concentration was $2.0 \mathrm{mg} / \mathrm{L}$, reactive species were already at excess level, resulting in pseudo-first order degradation kinetics of pharmaceuticals. When lower initial pharmaceuticals concentrations were applied, the reactive species to target pollutant ratio was further increased, therefore the pharmaceuticals degradation still followed the same pseudo-first order kinetics model. Such results suggest that although concentrations of such pharmaceuticals are usually at relatively low level in waste streams, the proposed MB/Redlight photosensitized pharmaceuticals degradation process can still be effective on site.

Regarding MB consumption (Figure 6. 2c), it showed a significant increase $(95 \%$ confidence interval) with increased initial pharmaceutical concentration. It reveals that, besides pharmaceuticals degradation by ${ }^{1} \mathrm{O}_{2}$, direct reactions among pharmaceuticals and $\mathrm{MB}$ can also occur simultaneously, which is most likely to be the reaction between DFN and excited MB, according to our previous work [65]. 
Chapter 6

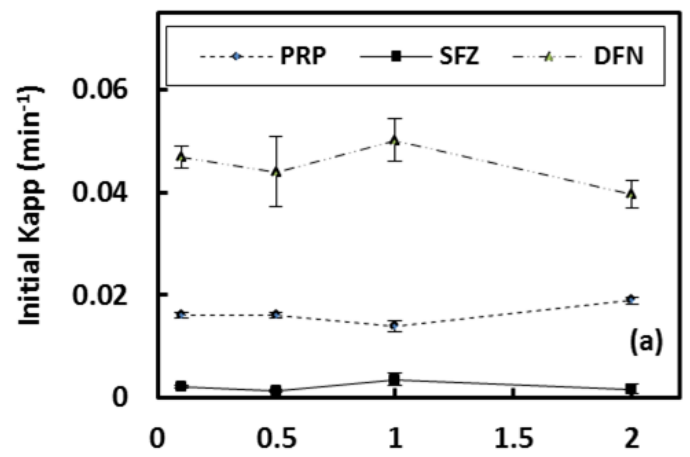

Initial pharmaceuticals concentration $(\mathrm{mg} / \mathrm{L})$

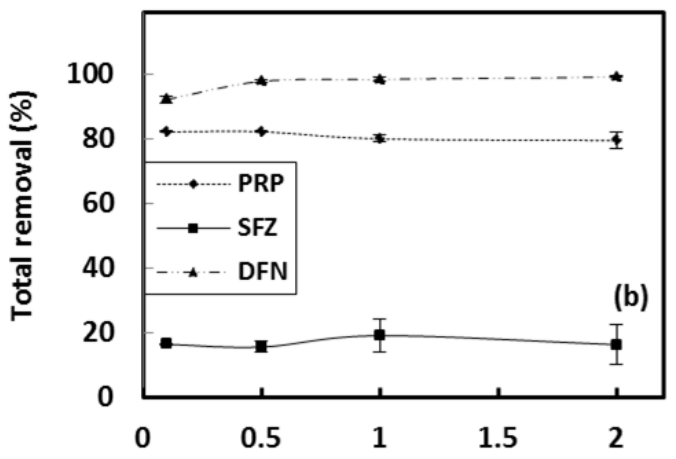

Initial pharmaceutical concentration $(\mathrm{mg} / \mathrm{L})$

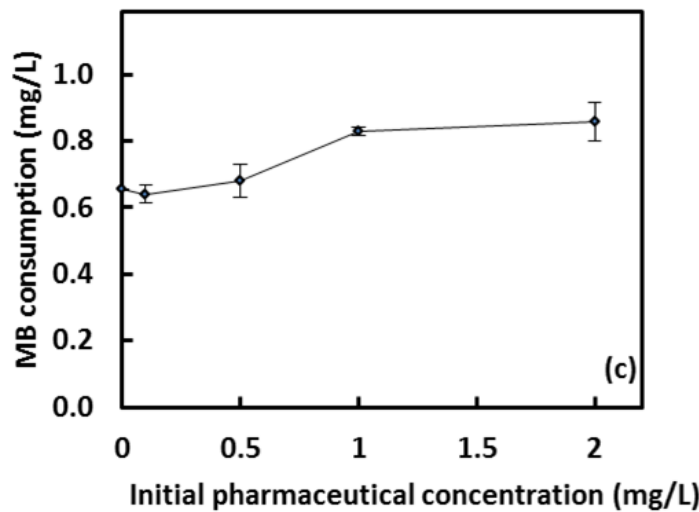

Figure 6. 2 Effect of initial pharmaceutical concentration on the initial degradation rate constants of pharmaceuticals (a), total removal after 120 minutes (b), and MB consumption (c). Initial MB concentration $=1.5 \mathrm{mg} / \mathrm{L}$, $\mathrm{pH}$ unadjusted, air equilibrated. 


\subsubsection{Effect of aeration rate}

Experiments with varied aeration rate were carried out to investigate the influence of aeration rate on the photosensitized degradation of pharmaceuticals. The optimized initial MB concentration of $1.5 \mathrm{mg} / \mathrm{L}$ was applied, the initial pharmaceutical concentration was $1.0 \mathrm{mg} / \mathrm{L}$, and $\mathrm{pH}$ was unadjusted. Air flow rate was varied from 0.0 to $5.0 \mathrm{~L} / \mathrm{min}$. Two groups of experiments were carried out, with initial dissolved oxygen concentration of 8.5 $\mathrm{mg} / \mathrm{L}$ or $2.0 \mathrm{mg} / \mathrm{L}$ respectively.

The results demonstrate that the varied air flow rate had no significant influence on degradation of pharmaceuticals, when the initial dissolved oxygen concentration was 8.5 $\mathrm{mg} / \mathrm{L}$ (Figure S6. 1). Degradation of PRP and SFZ was constant with the varied aeration rate from 0.0 to $5.0 \mathrm{~L} / \mathrm{min}$. Varied air flow rate from 0.5 to $5.0 \mathrm{~L} / \mathrm{min}$ did not affect DFN degradation, while slightly decreased degradation rate constants and total removal were obtained when no aeration was applied. Meanwhile, the dissolved oxygen concentration did not drop dramatically, so the decrease in degradation of DFN can be attributed to the limitation of mixing when no aeration was applied. Figure 6.3 shows the results with initial dissolved oxygen concentration of $2.0 \mathrm{mg} / \mathrm{L}$. With lower initial dissolved oxygen concentration $(2.0 \mathrm{mg} / \mathrm{L})$, degradation rate constants of PRP and SFZ were still constant when aeration rate varied from 0.0 to $5.0 \mathrm{~L} / \mathrm{min}$. The DFN degradation rate constants were independent on the increased aeration rate from 0.5 to $5.0 \mathrm{~L} / \mathrm{min}$, but much higher degradation rate constant $\left(0.085 \mathrm{~min}^{-1}\right)$ was obtained when no aeration was applied.

This could be explained by the two different types of MB photosensitization. It has been proposed by previous studies [186] that MB photosensitization involves two possible mechanisms. By absorbing energy from photons, MB molecules can be activated to triplet excited state (the more stable excited state of MB). Subsequently, the triplet excited state $M B$ can directly react with substrates via electron/hydrogen transfer, which is defined as Type I photosensitization. Alternatively, Type II photosensitization could take place: when ground state oxygen molecules are available, energy transfer between triplet MB and those ground state oxygen molecules would take place to generate an energy-rich form of oxygen, i.e. singlet oxygen, and then the singlet oxygen can react with substrates. Our previous work has demonstrated that $\mathrm{MB} /$ Red-light photosensitized degradation of DFN can be a result of 


\section{Chapter 6}

both mechanisms. Under identical other conditions, degradation of DFN via Type I processes is faster than that via Type II processes. In a previous section of the present work (section 6.3.3.2), we found that under aerobic conditions DFN degradation underwent both Type I and Type II processes, as can be explained as follows. When low initial dissolved oxygen concentration $(2.0 \mathrm{mg} / \mathrm{L})$ and no aeration were applied, the dissolved oxygen concentration in reaction solution remained at low level, and therefore the availability of oxygen molecules for quenching excited state MB decreased. As a consequence, more DFN molecules could undergo degradation involving the faster Type I process, so the overall degradation of DFN experienced a strong increase.
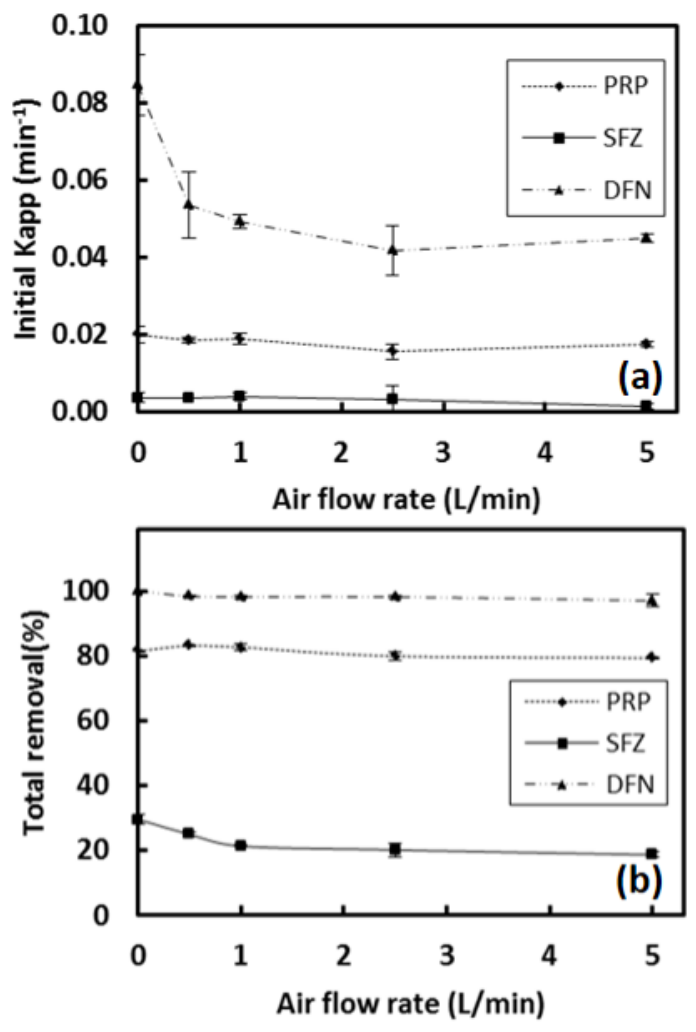

Figure 6. 3 Effect of air flow rate on the initial degradation rate constants of pharmaceuticals (a), total removal after 120 minutes (b). Initial MB concentration = $1.5 \mathrm{mg} / \mathrm{L}$, initial pharmaceutical concentration $=1 \mathrm{mg} / \mathrm{L}, \mathrm{pH}$ unadjusted, initial dissolved oxygen concentration $2 \mathrm{mg} / \mathrm{L}$. 


\subsubsection{Effect of $\mathrm{pH}$ on the transformation pathways of diclofenac}

In a recent study conducted by the authors, we reported that the $\mathrm{pH}$ imposes a significant impact on the $\mathrm{MB} /$ red-light sensitized pharmaceuticals degradation [65]. Higher $\mathrm{pH}$ greatly favoured the degradation of SFZ and PRP, both the degradation rate constants and total removal of those two compounds increased with increasing $\mathrm{pH}$. It is also found that, although the degradation of DFN was promoted under both neutral and alkaline conditions, highest DFN degradation rate was obtained under neutral conditions. It is of interest to have a better understanding of the effect of $\mathrm{pH}$ on DFN degradation. Therefore, experiments were conducted to examine this aspect via identifying DFN transformation products under different $\mathrm{pH}$ conditions by LC-MS/MS.

Several peaks at different retention times (RT) were observed by LC-MS/MS. Based on retention times and $\mathrm{m} / \mathrm{z}$ ratios observed, the results suggest that several smaller and more polar compounds were formed as a result of DFN degradation. In this study we focused on the main transformation products. The overview of all main transformation products detected in this study, together with their retention times and mass to charge ratios $(\mathrm{m} / \mathrm{z})$ are summarized in Table 6.1 , and the tentative structures are shown in Table S6. 2. The tentative transformation products are numbered in the order of their retention times.

The results demonstrate a significant role of $\mathrm{pH}$ on the formation of DFN transformation products (as shown in Table 6. 1). Five TPs were formed under both neutral and alkaline conditions. Under alkaline conditions, formation of TP4 was nearly two times higher than its formation under neutral conditions (Figure S6. 3). Under alkaline conditions, the hydroxyl ions in the reaction solution were much more abundant than that under neutral conditions. After decarboxylation of DFN the hydroxylation, the follow-up step to form TP4, i.e. addition of hydroxyl group, was promoted; as a consequence more TP4 was formed. TP6, TP8, and TP10, were also formed in more abundant amount under alkaline conditions compared with neutral conditions. 
Table 6. 1 Overview of the main DFN transformation products

\begin{tabular}{|c|c|c|c|c|}
\hline $\begin{array}{c}\text { Transformation } \\
\text { products }\end{array}$ & $m / z$ & $\mathrm{RT}(\min )$ & $\begin{array}{l}\text { Formation under } \\
\text { neutral conditions }\end{array}$ & $\begin{array}{l}\text { Formation under } \\
\text { Alkaline conditions }\end{array}$ \\
\hline TP1 & 234 & 1.76 & - & $\mathrm{V}$ \\
\hline TP2 & 234 & 2.61 & - & v \\
\hline TP3 & 244 & 3.97 & $v$ & - \\
\hline TP4 & 266 & 4.00 & v & v \\
\hline TP5 & 234 & 4.44 & $v$ & v \\
\hline TP6 & 266 & 4.49 & $v$ & v \\
\hline TP7 & 260 & 4.98 & v & - \\
\hline TP8 & 247 & 5.13 & v & v \\
\hline TP9 & 232 & 6.14 & - & v \\
\hline TP10 & 260 & 8.00 & v & v \\
\hline
\end{tabular}

TP1, TP2, and TP9 were only formed under alkaline conditions, while TP3 and TP7 were only formed under neutral conditions. The transformation from TP4 to TP1 and the transformation from DFN to TP2 can only be achieved under alkaline conditions. The routes leading to formation of TP9 from TP4 and TP10 are also only active under alkaline conditions. The transformation route from DFN to TP7 and the route from TP10 to TP3 are only active under neutral conditions. 


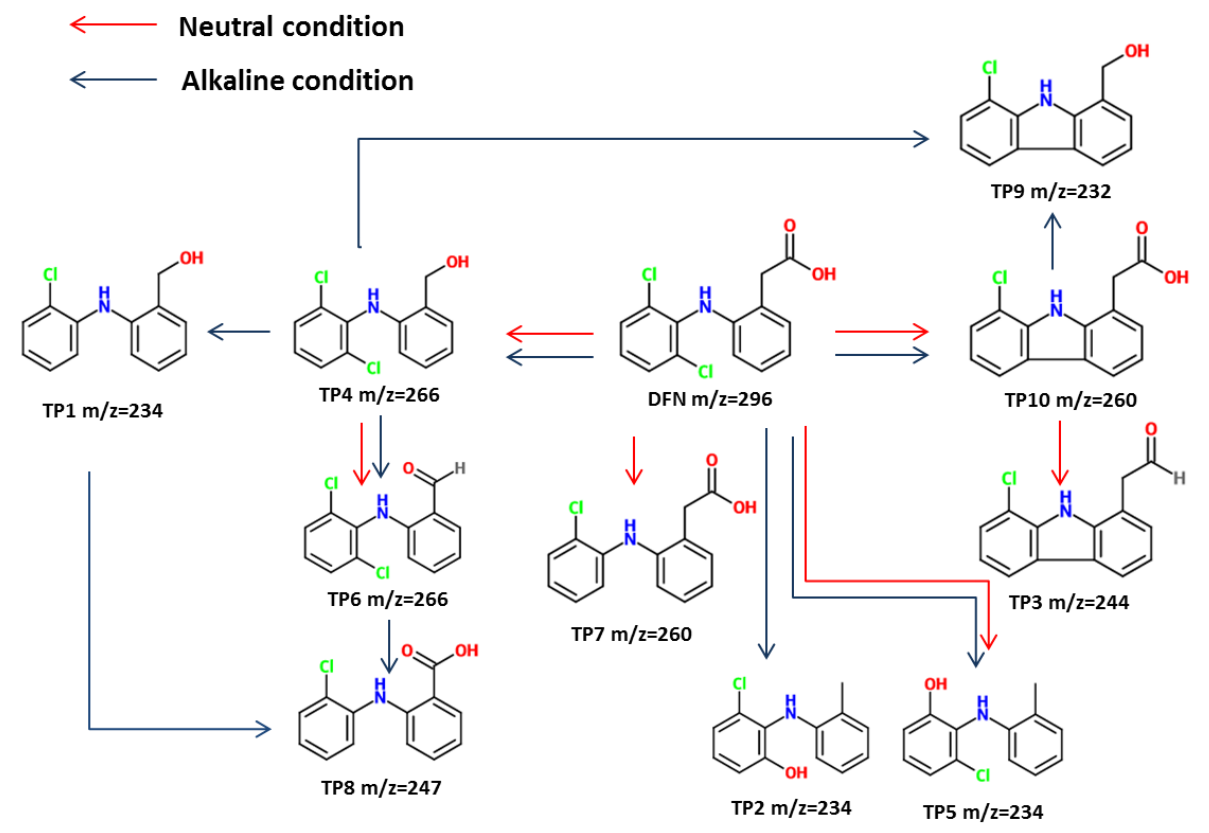

Scheme 6. 1 Proposed transformation pathways of DFN degradation

From the results obtained herein, we propose the following transformation pathways for DFN degradation. Diclofenac can lose one of its chlorine atom and a hydrogen atom, followed by structural rearrangement, leading to close of the middle ring and resulting the formation of a mono-halogenated carbazole structure (TP10, $\mathrm{m} / \mathrm{z}=260$ ). This structure and the process have been reported in several previous studies [91, 241, 252, 253]. The transformation from DFN to TP10 was greatly promoted under alkaline conditions. Subsequently, under alkaline conditions, substitution of carboxylic group by hydroxyl group took place and led to formation of TP9 $(\mathrm{m} / \mathrm{z}=232)$. Some authors proposed the transformation route of TP10 to TP9 to be a multi-step process, where the 1-chloro-9methyl-9H-carbazole was formed as the intermediate by decarboxylation of TP10 as the first step, followed by further transformation of this intermediate to TP9 with the participation of oxygen [241]. On the contrary, this intermediate was not observed in our study; the possible explanation is that this intermediate is not detectable in the measurement methods applied in the present study. Alternatively, under alkaline conditions, TP3 $(\mathrm{m} / \mathrm{z}=244)$ can also form from TP10, by reduction of the carboxylic group to aldehyde group, which has been described elsewhere [253]. 


\section{Chapter 6}

Instead of loss of a chlorine atom as the first step, DFN can undergo decarboxylation with addition of a hydroxyl group to form TP4 $(\mathrm{m} / \mathrm{z}=266)$ as the starting point of other consecutive processes. Although this transformation could take place under both neutral and alkaline conditions, the alkaline condition is more favourable. Subsequently, TP4 can undergo further transformation via three routes. Firstly, under alkaline conditions, TP9 were formed from TP4 by loss of one chlorine atom and a hydrogen atom. Alternatively, oxidation of the hydroxyl group in TP4 took place and led to formation of TP6 $(\mathrm{m} / \mathrm{z}=266)$, which has reduced hydrophilic character (longer retention time). Further transformation of TP6 led to formation of TP8 $(\mathrm{m} / \mathrm{z}=247)$, by further oxidation of the aldehyde group to carboxylic group and loss of a chlorine atom under alkaline conditions. The second route is in agreement with a previous study conducted by Martinez et al. [6], where intermediates of DFN degradation in a heterogeneous photocatalysis system were studied. Singlet oxygen was thought to play a vital role according to their study. A study conducted by Agüera et al. aiming at the use of TOF-MS analysis on phototransformation products of DFN reported the formation of TP6 [253], and another study conducted by Bartels and von Tümpling [254] examining the solar radiation influence on the decomposition process of DFN in surface waters reported the formation of TP8. In the third route, mono-halogenation took place as first step to form TP1 (m/z $=234)$, followed by oxidation of the hydroxyl group to carboxylic group to form TP8.

Alternatively, TP7 was formed from DFN by loss of a chlorine atom and addition of a hydrogen atom to the same spot, under neutral conditions. Loss of one chlorine atom led to an increase in hydrophilicity of TP7, which has shorter retention time compared with DFN. The tentative structure of TP7 has been described previously by other researchers [252] and those authors explained the formation of such compound by electron reduction. In addition, two isomers share the same $\mathrm{m} / \mathrm{z}$ of 234 , i.e. TP2 and TP5, were formed from the parent compound DFN by mono-dehalogenation and addition of a hydroxyl group. 


\subsection{Conclusions}

The effect of operational parameters on the pharmaceuticals degradation during $\mathrm{MB} /$ Red-light treatment was studied in the present study. The conclusions can be drawn from this study are summarized as follows:

* The degradation rate constants and total removal of pharmaceuticals were greatly affected by MB concentration. Aeration rate and initial pharmaceutical concentration had no significant impact on degradation of pharmaceuticals.

* Degradation rate constants and total removal of pharmaceuticals increased with increasing initial $\mathrm{MB}$ concentrations from $0.1 \mathrm{mg} / \mathrm{L}$ to $1.5 \mathrm{mg} / \mathrm{L}$, but further increased initial MB concentration from $1.5 \mathrm{mg} / \mathrm{L}$ to $5 \mathrm{mg} / \mathrm{L}$ had no significant impact.

* Initial pharmaceutical concentrations had no negative impact, suggesting that the proposed $\mathrm{MB} /$ Red-light process can still be effective in real waste streams where concentration levels of pharmaceuticals are relatively low.

* Even at low initial dissolved oxygen concentration, aeration is not an essential factor to achieve rapid degradation of the model-compounds. This result exhibits potential for energy and costs saving in real applications, because aeration requires additional costs and in-put energy.

* The $\mathrm{pH}$ conditions impose a significant impact on the transformation pathways of DFN.

\subsection{Acknowledgements}

This work was performed in the cooperation framework of Wetsus, European Centre of Excellence for Sustainable Water Technology (www.wetsus.eu). Wetsus is cofunded by the Dutch Ministry of Economic Affairs and Ministry of Infrastructure and Environment, the Province of Fryslân, and the Northern Netherlands Provinces. The authors would like to thank the participants of the research theme "Advanced water treatment" for the fruitful discussions and their financial support. The authors gratefully acknowledge Ton van der Zande for the realization of the instrumental analysis, and Sofia SemitsoglouTsiapou for the fruitful discussions. 
Chapter 6

Supplementary information

Table S6. 1 Chemical structure of the model compounds

Compound Structure


Chapter 6

Table S6. 2 Overview of the main transformation products of DFN

Product Product


Chapter 6
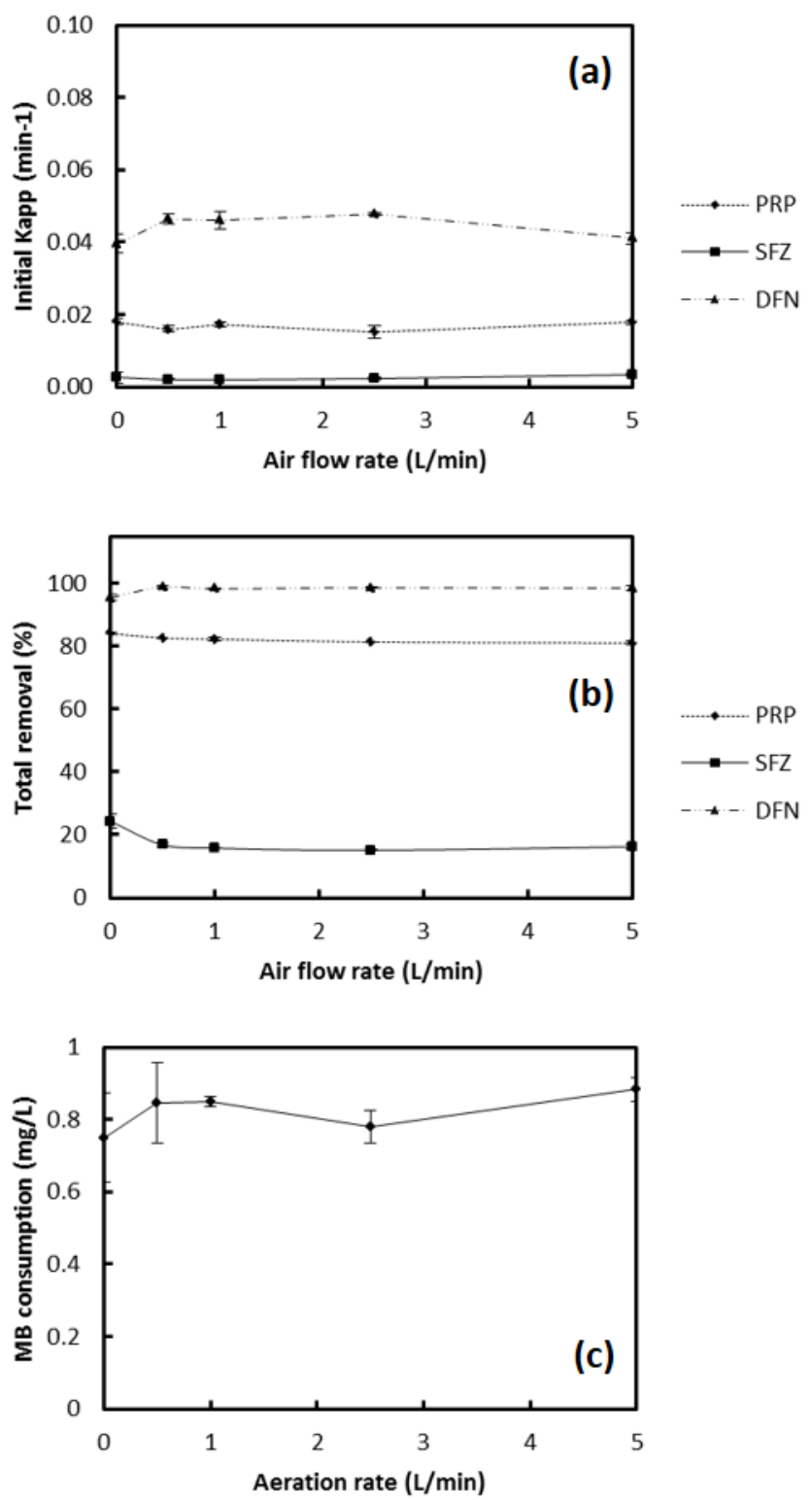

Figure S6. 1 Effect of air flow rate on the initial degradation rate constants of pharmaceuticals (a), total removal after 120 minutes (b), and MB consumption (c). Initial $\mathrm{MB}$ concentration $=1.5 \mathrm{mg} / \mathrm{L}$, initial pharmaceutical concentration $=1 \mathrm{mg} / \mathrm{L}, \mathrm{pH}$ unadjusted, initial DO is $8.5 \mathrm{mg} / \mathrm{L}$. 
Chapter 6
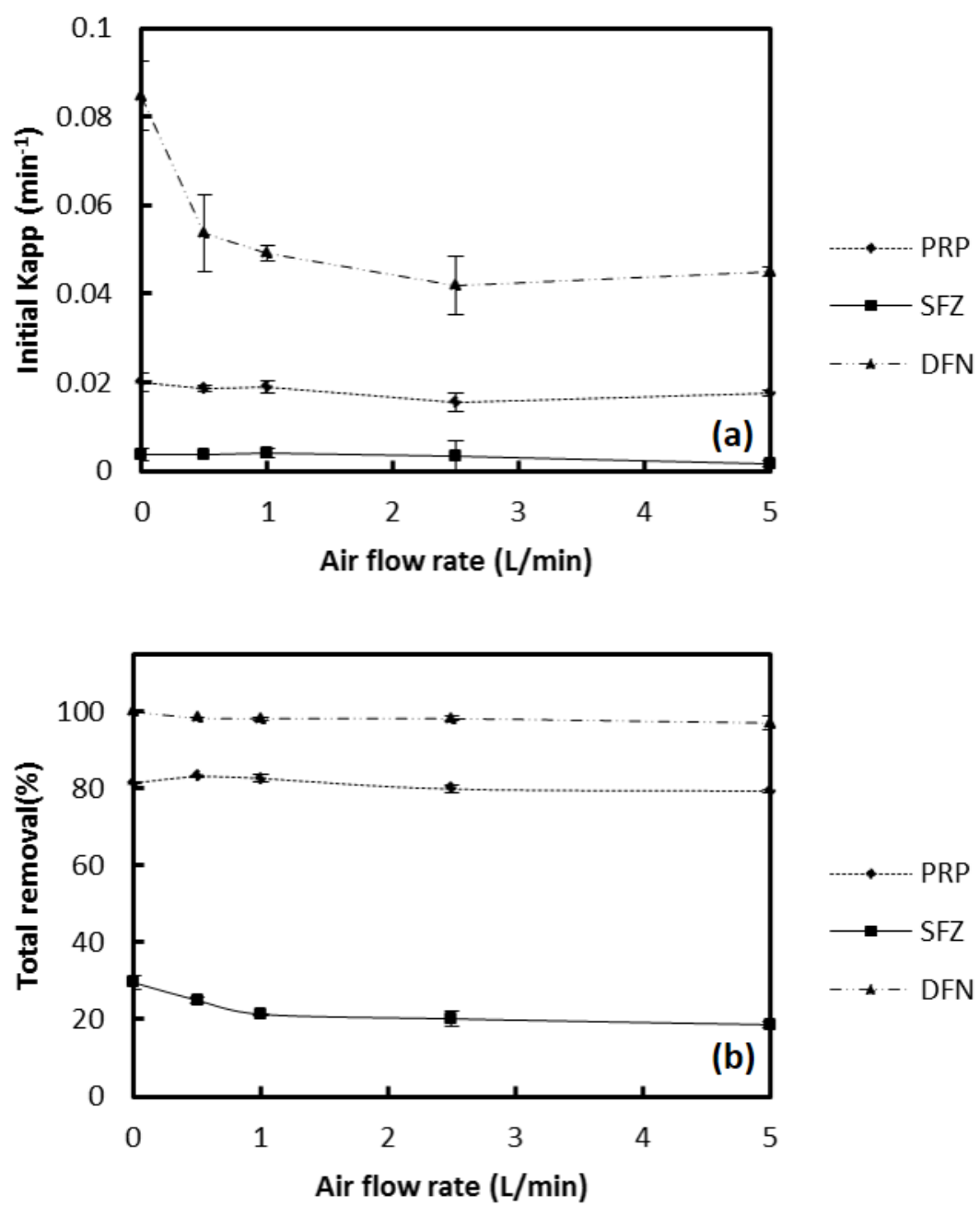

Figure S6. 2 Effect of air flow rate on the initial degradation rate constants of pharmaceuticals (a), total removal after 120 minutes (b). Initial MB concentration = $1.5 \mathrm{mg} / \mathrm{L}$, initial pharmaceutical concentration $=1 \mathrm{mg} / \mathrm{L}$, $\mathrm{pH}$ unadjusted, initial dissolved oxygen concentration $2 \mathrm{mg} / \mathrm{L}$. 
Chapter 6

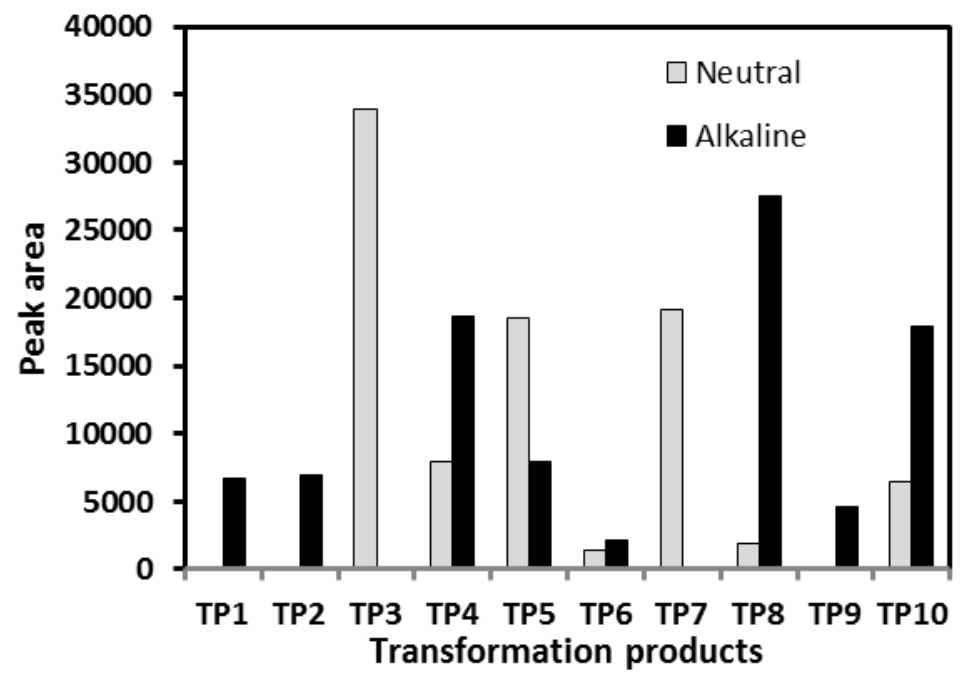

Figure S6. 3 Formation of TPs under different $\mathrm{pH}$ conditions. Peak area is used as the indication of abundance of the TPs. 


\section{Chapter 7}

General discussion

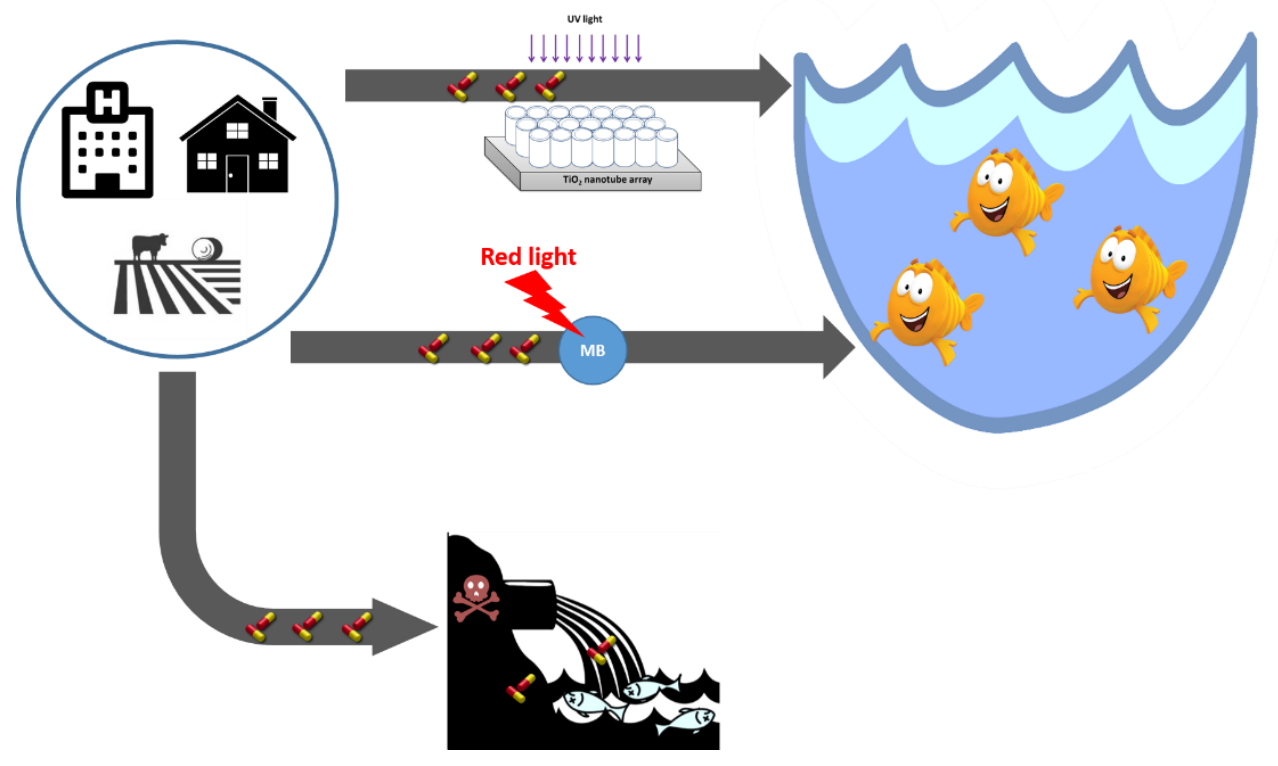




\section{Chapter 7}

\subsection{Introduction}

The occurrence of micropollutants in the environment, the risks associated with their presence, and the insufficient efficacy of conventional WWTPs on their removal is demanding for more efficient treatment techniques to safe-guard the quality of the aquatic environment and water supply. Given this context, the general objective of this thesis is to contribute to a better understanding and further development of photochemical treatment processes for micropollutant removal from aqueous streams. Emphasis of the experimental work of this thesis is given to two topics: (1) Further development of UV/TiO 2 technique by application of TNA as immobilized catalyst; (2) Development of alternative photochemical water treatment technique for micropollutant removal from wastewater. The main research outcomes of previous chapters are summarized in this chapter. Accordingly, challenges and opportunities in future research and application are identified.

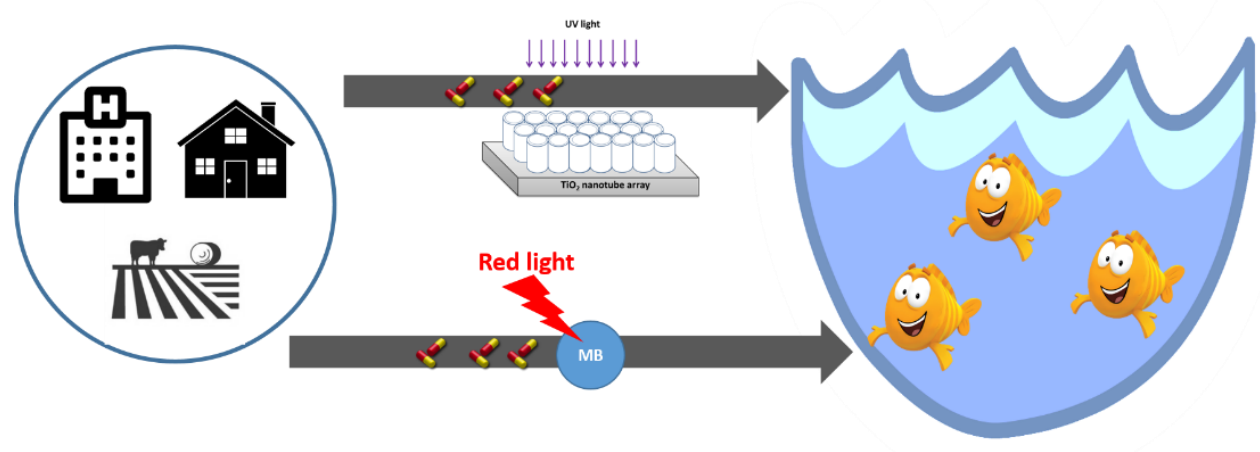

Figure 7. 1 Photochemical micropollutant removal techniques studied in this thesis

\subsection{Main outcomes of this thesis}

\subsubsection{Use of $\mathrm{TiO}_{2}$ nanotube array as immobilized photocatalyst}

The application of $\mathrm{TiO}_{2}$ for pollutants elimination has received enormous research interests [110-114]. Many research works at lab-scale have been carried out on removal of micropollutants from water using suspension of $\mathrm{TiO}_{2}$ nanoparticles $[61,107,141-143]$. The use of $\mathrm{TiO}_{2}$ nanoparticles is beneficial for fundamental research at lab-scale due to their high surface area which allows for good mass transfer, but not favourable in real life implications [151]. Firstly, although $\mathrm{TiO}_{2}$ is considered to be non-toxic to humans, leaked 
$\mathrm{TiO}_{2}$ nanoparticles in the environment have been considered to impose adverse impacts to living species $[257,258]$. Thus, the effluent should be treated in solid liquid separation device, e.g. membrane filtration, to achieve separation and retention of the $\mathrm{TiO}_{2}$ nanoparticles. Secondly, the catalyst particles are unstable in complex water matrices. For instance, it has been reported that $\mathrm{TiO}_{2}$ nanoparticles tend to form aggregates in water with increased levels of alkalinity [144]. Given this context, immobilized $\mathrm{TiO}_{2}$ would be more favoured in full scale implementations than suspended $\mathrm{TiO}_{2}$ nanoparticles. The $\mathrm{TiO}_{2}$ nanotube array (TNA) is a promising form of immobilized $\mathrm{TiO}_{2}$ due to its facile fabrication procedures, tuneable properties, and relatively large surface area compared to other form of immobilized $\mathrm{TiO}_{2}$. Although a few studies have been conducted to apply TNA for micropollutant removal [165], numerous knowledge gaps still exist. For instance, few studies documented the effect of TNA tube length on micropollutant removal [165], but effects of other TNA properties (e.g. tube diameter, crystallographic composition) on micropollutant removal have not been well studied. Moreover, the influence of water quality parameters and water matrices still demand investigation. In this thesis, the application of TNA for photocatalytic micropollutant removal was evaluated (Chapter $\mathbf{2}$ and Chapter 3).

In Chapter 2, the surface morphology and crystallographic composition of the TNA were found to impose crucial influence on its photocatalytic micropollutant removal performance: For TNAs with given tube diameter, the degradation of the model micropollutant was favoured when a mixture of anatase and rutile was present in the TNAs structure; for TNA with certain crystallographic composition, rapid micropollutant degradation was obtained when the nanotubes diameter reached certain threshold value. Unlike the $\mathrm{TiO}_{2}$ nanoparticles, TNA is stable in a broad $\mathrm{pH}$ range $(3-11)$. The primary micropollutant degradation route in TNA based system involves hydroxyl radical attack and takes place in the liquid phase. The presence of some common inorganic ions, e.g. bicarbonate, phosphate, were found to have no notable adverse impact on the TNA based system, while the presence of NOMs were found to be inhibitory.

Such inhibitory effect of NOMs has been well documented in literature for $\mathrm{TiO}_{2}$ nanoparticle based systems. For instance, Brame et al. reported the presence of NOMs 


\section{Chapter 7}

inhibited the performance of $\mathrm{TiO}_{2}$ slurry system, and their mechanistic study suggested that the competitive adsorption by NOMs and reactive oxygen species (ROS) scavenging were the most influential mechanisms [212]. TNA have different properties (e.g. specific surface area, hydrophilicity) compared to $\mathrm{TiO}_{2}$ nanoparticles, which change the interactions between TNA and the background water constituents, but little is known about the effect of NOMs on TNA based photocatalytic system in literature. In addition, the presence of NOM in water bodies is associated with the presence of inorganics. For example, phosphate, sulfate, bicarbonate, chloride, etc., are the most commonly present inorganic anion species in a broad range of water matrices. The photocatalytic removal of micropollutants by $\mathrm{TiO}_{2}$ photocatalytic processes can also be affected by those co-existing inorganic anions by competitive adsorption and interaction with ROS [168, 174, 215]. In this context, the presence of co-existing inorganic anions may impose an impact on the effect of NOMs on the photocatalytic system. In a recent study by Long et al., reduced inhibitory effect of humic acids on photocatalytic performance of $\mathrm{TiO}_{2}$ particles by the presence of phosphate was reported [142]. However, the combined effects of NOMs and other commonly present inorganic anions have not been well documented in literature. Therefore, the effect of NOMs, and the combined effects of NOMs and co-existing inorganic ions on the micropollutant removal over TNA were studied in depth in Chapter 3. The results show that, although NOMs in the bulk liquid phase can undergo photosensitization upon absorption of UV light, which is beneficial for removal of some micropollutant, the overall effect of NOMs on the micropollutant removal over TNA is detrimental. Such overall inhibitory effect could be mitigated under acidic or alkaline conditions. The acidic $\mathrm{pH}$ strengthened both the interaction between TNA and target contaminants and the photosensitization effect of NOMs; while alkaline conditions reduced NOMs adsorption on the TNA surface. The presences of bicarbonate and phosphate anions also mitigate the inhibitory effect of NOMs, by reducing $\mathrm{h}^{+}$scavenging of NOMs.

\subsubsection{Photocatalytic fuel cell as inspiration to improve micropollutant removal performance}

In $\mathrm{TiO}_{2}$ photocatalysis, upon radiation of proper photon energy, $\mathrm{e}^{-}$and $\mathrm{h}^{+}$are generated, which can induce production of ROS and subsequently the degradation of 
micropollutants. However, many of the $\mathrm{e}^{-} / \mathrm{h}^{+}$pairs do not participate in such reactions but recombine directly, generating only heat (which is a loss of energy). The fast recombination of $\mathrm{e}^{-} / \mathrm{h}^{+}$pairs leads to a reduced quantum yield efficiency of $\mathrm{TiO}_{2}$ photocatalysis. In this thesis, a strategy that can significantly improve the efficiency of $\mathrm{TiO}_{2}$ photocatalytic micropollutant removal was evaluated. The obtained results (Chapter 4 ) suggest that, by simply connecting the TNA to a copper cathode (to form a PFC system), the potential difference between the two electrodes can effectively extract the photo generated electrons from the TNA to the copper cathode and better micropollutant removal could be obtained. Such enhancement was observed in a broad pH range $(3-11)$, and the highest enhancement was obtained under acidic $\mathrm{pH}$ conditions. Application of the PFC system under neutral $\mathrm{pH}$ conditions in tap water resulted in twice as fast degradation of the model micropollutant (MCPA) compared to the conventional photocatalysis system, when the same TNA was used as catalyst and all other operational parameters were kept identical. Under optimal pH conditions, good micropollutant removal efficiency could be obtained even for WWTP effluent: at the optimal pH, MCPA removal kinetics in the PFC system was about 1.5 times faster than that in the conventional photocatalysis system. Mechanistic studies demonstrated that the enhancement in micropollutant removal performance can be attributed to two phenomena: (1) better $\mathrm{e}^{-} / \mathrm{h}^{+}$separation; (2) production and participation of more ROS.

\subsubsection{Application of $\mathrm{MB} /$ Red-light as alternative micropollutant removal technique}

The primary ROS in most existing photochemical micropollutant removal techniques, e.g. $\mathrm{UV} / \mathrm{Cl}_{2}, \mathrm{UV} / \mathrm{O}_{3}, \mathrm{UV} / \mathrm{HOCl}, \mathrm{UV} / \mathrm{ClO}_{2}, \mathrm{UV} / \mathrm{H}_{2} \mathrm{O}_{2}$, photo-Fenton, and $\mathrm{UV} / \mathrm{TiO}_{2}$ are radicals like $\cdot \mathrm{OH}$ and $\cdot \mathrm{Cl}$. The strong oxidizing power of these radicals enabled the applications of such techniques for various purposes, including organic pollutant removal, disinfection etc. However, the high oxidizing power also makes those ROS less selective: it is well documented that the presence of background dissolved organic matter can significantly hinder the removal of target micropollutants [174] due to quenching of radicals. Viewing this, studies have been carried out to employ photosensitization processes, where more selective ROS (i.e. ${ }^{1} \mathrm{O}_{2}$ ) is produced, for organic pollutants removal in various water 


\section{Chapter 7}

matrices $[64,129,130]$. Among various photosensitization processes, the red light induced methylene blue photosensitization (MB/Red-light) is a feasible alternative for micropollutant removal purpose. $\mathrm{MB}$ has strong light absorption in the visible range (red light) which can avoid the "inner filter" effect of NOMs in complex water matrices, has a relatively high quantum yield of ${ }^{1} \mathrm{O}_{2}$ formation [183], and has a long history of safe use in human treatment without major side effect. It has been applied for disinfection in not only wastewater treatment but also blood products [184-189], where singlet oxygen was generated to kill pathogens, as well as medical therapy, where red light induced MB photosensitized singlet oxygen was directed to selectively kill tumour cells for cancer therapy [187]. Nevertheless, its micropollutant removal efficacy has not been examined before. Therefore, the application of red light induced methylene blue photosensitization (MB/Red-light) process for micropollutant removal was evaluated (Chapter $\mathbf{5}$ and Chapter 6). It is found that the MB/Red-light photosensitization is capable for micropollutant removal, but this method also showed selectivity to certain kind of micropollutants: among the four tested model micropollutants, diclofenac, propranolol and sulfamethoxazole were able to be removed by $\mathrm{MB} /$ Red-light, while metoprolol was persist. Singlet oxygen was found to be the crucial ROS, while another additional mechanism, i.e. a direct reaction with triplet $\mathrm{MB}$, could also contribute to removal of some target pollutants. Common back ground water constituents (bicarbonate, phosphate, and dissolved natural organic matter) were found to have no noticeable detrimental effect, which suggests that the $M B /$ red-light system has great potential to be used in real wastewater. Higher $\mathrm{pH}$ was found to impose positive impact on the efficacy of the $\mathrm{MB} /$ red-light system. $\mathrm{MB}$ degradation was observed along with the target pollutants during the MB/Red-light treatment process. The MB dosage was found to impose an impact on the micropollutant removal performance.

\subsection{Outlook}

\subsubsection{Move towards full scale application}

This thesis contributes to a better understanding of the application of TNA for photocatalytic micropollutant removal and development of $\mathrm{MB} /$ Red-light as an alternative micropollutant removal technique. For full scale applications, efforts are needed. Thus, some directions for possible further research work are suggested in this section. 


\subsubsection{Stability of TNA after long time exposure to complex water matrices and to complex hydraulic conditions}

The use of TNA enables good separation and reuse of the photocatalyst in real life implementations. The obtained results in this thesis (Chapter $\mathbf{2}$ ) indicated its stability after several times of repeated use. However, in full scale water treatment practices, the photocatalyst would be exposed to the water constituents in much longer time. Some back ground water constituents are known to be able to interfere with the photocatalysts by adsorption. For instance, the commonly present background organic matter in water bodies imposes significant inhibitory effect on the micropollutant removal performance of $\mathrm{TiO}_{2}$ photocatalytic techniques, because they can screen light, scavenge ROS, and block the active site of the $\mathrm{TiO}_{2}$ surface $[212,235]$. Up till now, most of the studies on the effects of background water constituents focused on direct effects, and did not give enough attention to long-term effects on the stability of the catalysts. Only a very recent work presented by Peng et al. suggested that the reactivity of $\mathrm{TiO}_{2}$ nanoparticles could change significantly after long exposure to natural water in ambient environment (without artificial UV light radiation), because: (1) a NOM layer can be formed on and cap the $\mathrm{TiO}_{2}$ particles surface via adsorption; (2) the adsorbed NOM layer can act as ROS scavenger and subsequently reduce the concentration of ROS in the bulk liquid phase [143]. Still, the experimental approach applied in that study can hardly represent the situation in full scale implementations: in full scale applications, the photocatalysts are continuously exposed to not only NOMs but also to light irradiation, and are continuously generating ROS, thus the development of the NOM layer might differ from that observed in the work of Peng et al. Therefore, it would be of great interest to investigate the stability of TNA in long time exposure to complex water matrices under operation. Moreover, the mechanical stability of TNA under different hydraulic conditions should also be documented. For instance, the hydraulic retention time (HRT) is an important parameter that affects the dimension of the reactor, which is a measure of the flow velocity. In real life applications, large catalyst surface area in a limited volume would be appreciated, so compact reactor with short HRT would be more favourable. However, the higher flow velocity usually results in bigger shear force at the catalyst surface. Therefore, it is important to examine the mechanical stability of TNA and document the bearable hydraulic conditions. These works would assist the water treatment 


\section{Chapter 7}

professionals to better understand the applicability of TNA in full scale applications, and assist the process design and cost estimation.

\subsubsection{By-products formation and toxicity test}

This thesis studied the $\mathrm{MB} /$ Red-light as an alternative micropollutant removal technique, which relies on the selective oxidation ability of ${ }^{1} \mathrm{O}_{2}$. Although its weaker oxidizing power (compared to radicals like $\cdot \mathrm{OH}, \cdot \mathrm{Cl}$ ) enabled its selectivity, it is difficult to achieve total mineralization of the target pollutants, thus the target pollutants are only partly degraded to other organic by-products (Chapter 5). Moreover, although $\mathrm{TiO}_{2}$ photocatalysis is able to achieve total mineralization of various organic pollutants, it is not economically favourable, so in most cases the $\mathrm{TiO}_{2}$ photocatalysis is also only aimed at transformation of the target pollutant. Therefore, before full scale application, in order to safe guard the environment and water supply, it is necessary to study the by-products formation and the change in total toxicity of the treated water with treatment time. Such information can help to evaluate and control the risks, and assist the process design for delivering of safer effluents.

\subsubsection{Exploring the limits of $M B /$ Red-light technique}

This thesis demonstrated the potential of $\mathrm{MB} /$ Red-light technique on micropollutant removal. To apply this technique in practice, some crucial knowledge gaps still exist. In Chapter 5, only four model micropollutants were tested in the proposed $\mathrm{MB} /$ Red-light technique, and it was found that only two of them were rapidly removed within the applied treatment time span, while one of them was found to be persistent. This is due to the selectivity of the primary oxidant $\left({ }^{1} \mathrm{O}_{2}\right)$ in the $\mathrm{MB} /$ Red-light system. According to literature, ${ }^{1} \mathrm{O}_{2}$ is reactive towards electron-rich moieties including polycyclic aromatic rings, benzene rings activated with electro-donating substituents, and conjugated double bonds [129]. Thus, more micropollutants with different structures should be studied to gain a bigger vision on the applicability of the MB/Red-light technique. Secondly, the effect of NOMs on MB/Red-light technique was studied in the presence of $10 \mathrm{mg} / \mathrm{L} \mathrm{NOM}$. In some wastewater streams, the background organic matter concentration can be even higher. Thus it is important to document the highest bearable background organic matter concentration level. 


\subsubsection{Process optimization and reactor design}

For full scale applications, there are still some important issues that need to be addressed. First of all, the experiments were conducted in batch mode in this thesis, and process and reactor optimization have not been explored, so the energetic efficiency was not optimal (Table 7. 1). Process optimization is needed to lower the energy consumption of the techniques studied in this thesis. More comprehensive studies on the effect of operational parameters are wanted to provide adequate information for process optimization. For both the application of TNA and the MB/Red-light technique, the effects of some operational parameters have been briefly investigated, but the approach in this thesis was single factor approach. Thus, the possible synergetic effects of multiple operational parameters could not be elucidated from the work reported in this thesis. Therefore, considering process optimization for full scale applications, more sophisticated experimental designs (e.g. response surface methodology) should be adopted to better understand the correlations between the micropollutant removal performance and the operational parameters.

For an effective photochemical process the distribution of light is an essential parameter. Thus, another important approach to improve the system performance is the optimization of reactor design to obtain good light distribution. One possible design is the flat UV/TNA reactor consisting of a UV-LED array and TNA. The flat reactor design can enable short radiation pathway that can minimize the "inner filter" effect of back ground organic matter. The UV-LED array can be constructed with large amount of low power UV-LEDs to gain more homogeneous light distribution within the reactor to maximize the light harvesting by TNA. A schematic representation of the flat LED reactor design is shown in Figure 7. 2. 
Table 7. 1 Radiant energy demand of the studied techniques

\begin{tabular}{|c|c|c|c|}
\hline Treatment technique & Target pollutant & Water matrices & Lowest RE/O* \\
\hline & MCPA & Pure water & 10.03 \\
\hline & MCPA & Tap water & 94.95 \\
\hline \multirow[t]{4}{*}{ UV/TNA } & MCPA & WWTP effluent & 11.43 \\
\hline & MTP & Pure water & 17.89 \\
\hline & MTP & Tap water & 37.89 \\
\hline & MCPA & Pure water & 3.52 \\
\hline \multirow[t]{3}{*}{ TNA-Cu PFC } & MCPA & Tap water & 42.56 \\
\hline & MCPA & WWTP effluent & 7.86 \\
\hline & DFN & Pure water & 0.34 \\
\hline \multirow[t]{2}{*}{ MB/Red-light } & PRP & Pure water & 10.46 \\
\hline & SFZ & Pure water & 33.98 \\
\hline
\end{tabular}

*Lowest RE/O: radiant energy required to achieve $90 \%$ removal of the pollutant $\left(\mathrm{kWh} / \mathrm{m}^{3}\right)$ under the most optimal conditions applied in this study.

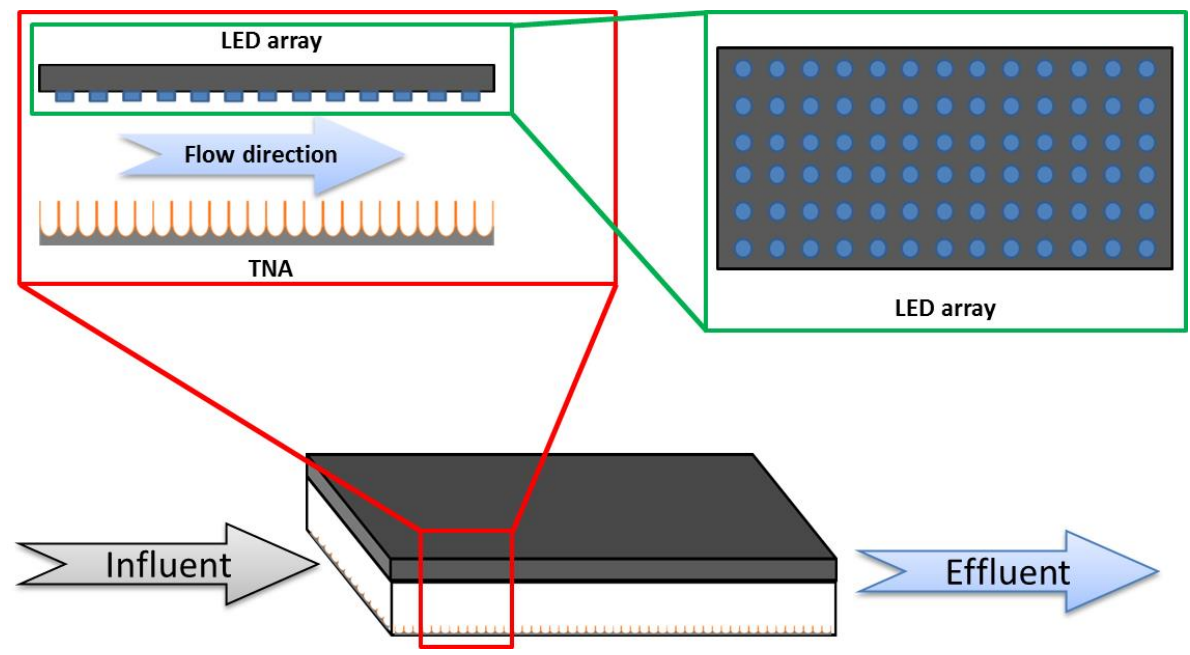

Figure 7. 2 Schematic of the flat LED array reactor 
Another possible design is the fluidized UV-LED bed reactor (Figure 7. 3). This concept was firstly proposed by Kuipers et al. [242]. In such kind of reactor, the LEDs are moving freely inside the reactor and wirelessly powered by induction, which can optimize the light distribution and penetration, and energy consumption. The TNA can fit well with this concept: TNA can be fixed to the UV-LED to construct the UV-LED/TNA unit that can moving freely inside the reactor. To examine the reactor designs proposed herein, firstly lab-scale reactor should be constructed and tested to obtain knowledge on the effects of important design parameters (e.g. HRT, flow rate etc.). Then a pilot scale reactor should be studied to evaluate the performance in real wastewater (for wastewater treatment purposes) or raw water (for tap water treatment purposes), to accumulate knowledge for better operation in full scale and further optimizing the operational parameters.

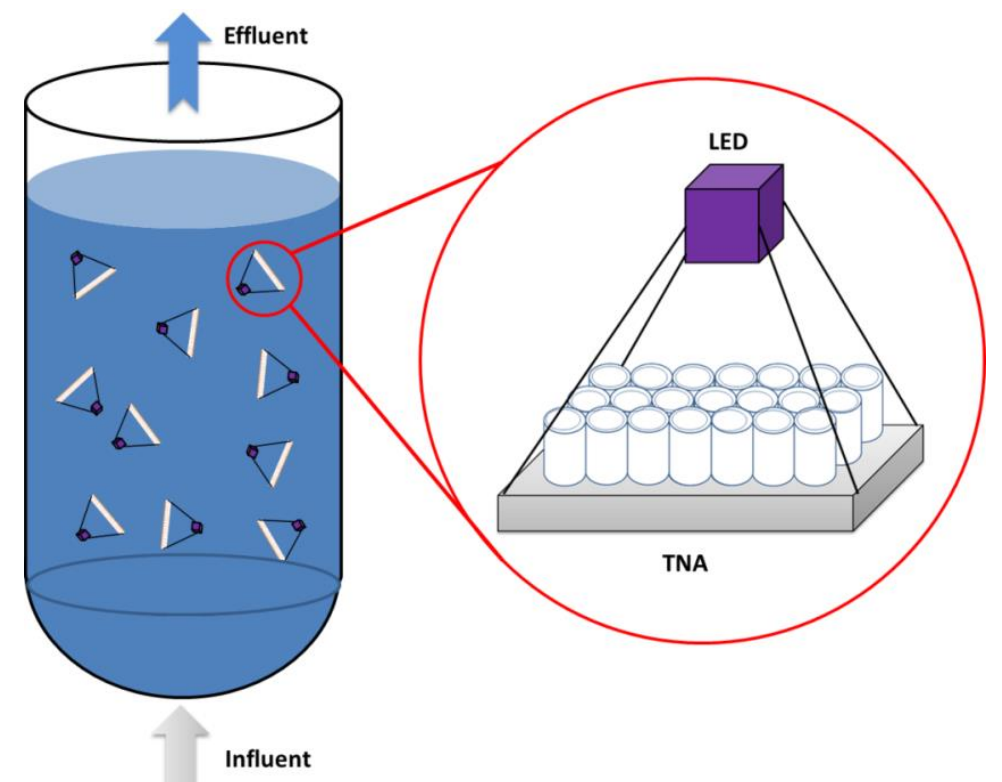

Figure 7. 3 Schematic of the fluidized LED bed reactor

\subsubsection{Use of solar light as light source}

From the economic perspectives, the artificial light sources are not very favourable. For instance, although the solid state lighting technology (LED) greatly increased efficiencies to convert electrical energy to light, the energetic efficiency is still low. For instance, for the red light LED used in this thesis it only converts around $22 \%$ input electrical power to radiant 


\section{Chapter 7}

power; and for the UV-LED used in this thesis it only converts around $26 \%$ input electrical power to radiant power. For conventional UV light sources like low pressure mercury gas discharge lamps and medium pressure mercury gas discharge lamps, their energetic efficiencies are around $32 \%$ and $12 \%$, respectively. Thus, more than half of the input electricity does not contribute to the pollutant removal. To overcome the need of artificial light sources, the use of solar light would be highly favourable.

For the $\mathrm{TiO}_{2}$ photocatalysis techniques, due to the wide band gap (3.0 eV for rutile and $3.2 \mathrm{eV}$ for anatase), the $\mathrm{TiO}_{2}$ absorbs light only in UV region ( $\lambda \leq 400 \mathrm{~nm}$ ). However, UV light accounts for only about $5 \%$ of the total solar spectrum, while more than $40 \%$ solar radiation are in the visible region [259]. Therefore, modification of $\mathrm{TiO}_{2}$ may be interesting, to extend its light absorption from UV region to visible region, in order to improve its photoresponse under solar light radiation. In recent years, many attempts have been made for this purpose and various strategies have been adopted, including doping of boron [125], nitrogen [260], carbon [112], sulphur [261-263], and formation of heterojunction with narrow band gap semiconductors [264]. Such modification methods could effectively enhance the visible light absorption of $\mathrm{TiO}_{2}$. However, the change in band structures usually leads to change in the primary ROS when applied to photocatalytic applications. According to Fotiou et al., the primary ROS in visible light photocatalysis changed from hydroxyl radicals to less powerful superoxide radical [112]. Thus, further research efforts are needed to obtain comprehensive understanding on the use of visible light active $\mathrm{TiO}_{2}$ for micropollutant removal. For instance, it is necessary to explore the applicability of visible light active $\mathrm{TiO}_{2}$ for different micropollutants. It is also important to investigate the performance of visible light photocatalysis in real complex water matrices under real solar light radiation. Process and reactor design is another essential topic that needs to be explored, to ensure maximized efficiency of solar light utilization and optimized system performance.

For the $\mathrm{MB} /$ Red-light photosensitization technique, due to the light absorbance property of the photosensitizer ( $\mathrm{MB}$ has good light absorption in the visible region, especially in the red range), it has great potential to employ solar light as light source. Further research works can be performed to explorer the performance of $\mathrm{MB}$ 
photosensitized micropollutant removal under sun light. Special focus can be given to process and reactor design, to lower the energy input for this technique and improve the micropollutant removal performance.

\subsubsection{Better utilization of photogenerated electrons in photocatalytic processes - integrating water treatment with resource recovery}

The use of PFC system can enhance the photocatalytic micropollutant removal process by facilitating the $\mathrm{e}^{-} / \mathrm{h}^{+}$separation (Chapter 4 ). The longer life time of the photogenerated electrons result in production of additional ROS which would also contribute to the enhancement of micropollutant removal efficiency. Apart from that, it has been documented that the redox potential of the electrons produced in $\mathrm{TiO}_{2}$ photocatalysis is sufficient to trigger reduction of $\mathrm{CO}_{2}$ to produce organic compounds. The application of $\mathrm{TiO}_{2}$ photocatalysis for $\mathrm{CO}_{2}$ reduction has received increasing attention $[265,266]$. The $\mathrm{CO}_{2}$ reduction by $\mathrm{TiO}_{2}$ photocatalysis relies on the photogenerated electrons, some feasible reaction pathways are listed below:

$$
\begin{aligned}
& \mathrm{CO}_{2}+2 \mathrm{H}^{+}+2 \mathrm{e}^{-} \rightarrow \mathrm{HCOOH} \\
& \mathrm{CO}_{2}+6 \mathrm{H}^{+}+6 \mathrm{e}^{-} \rightarrow \mathrm{CH}_{3} \mathrm{OH}+\mathrm{H}_{2} \mathrm{O} \\
& \mathrm{CO}_{2}+8 \mathrm{H}^{+}+8 \mathrm{e}^{-} \rightarrow \mathrm{CH}_{4}+2 \mathrm{H}_{2} \mathrm{O}
\end{aligned}
$$

Among them, the formations of methane and methanol are thermodynamically more favourable (-0.24 V vs. NHE and $-0.38 \mathrm{~V}$ vs. NHE for methane and methanol production, respectively) than the formation of formic acid (-0.61 V vs. NHE). However, from the kinetics point of view, the formations of methane and methanol are more difficult than the formation of formic acid, because more electrons are required for the formation of methane and methanol [266].

In $\mathrm{TiO}_{2}$ photocatalytic $\mathrm{CO}_{2}$ reduction processes, $\mathrm{h}^{+}$and $\cdot \mathrm{OH}$, which are the primary oxidant for organic pollutants removal processes, are detrimental and need avoidance. Thus, the pollutants removal processes and the $\mathrm{CO}_{2}$ reduction processes are theoretically compatible in the PFC system. It would be highly appreciable to couple such two processes, to make the best use of the input photon energy. A schematic representation of this concept 


\section{Chapter 7}

is shown in Figure 7. 4. In such concept, the anode and the cathode compartment can be spatially separated by a membrane to avoid any possible interference between the two processes. In the anode compartment, the photogenerated $\mathrm{h}^{+}$and $\cdot \mathrm{OH}$ are employed for organic micropollutant removal or disinfection; whereas the photogenerated electrons are directed to the cathode and be utilized in $\mathrm{CO}_{2}$ reduction. One of the possible application scenarios of the cathode $\mathrm{CO}_{2}$ reduction is upgrading of biogas from the anaerobic digestion processes. Further research efforts can be taken to investigate the possible synergetic effect of integrating such two processes to obtain optimized micropollutant removal performance and maximized $\mathrm{CO}_{2}$ conversion.

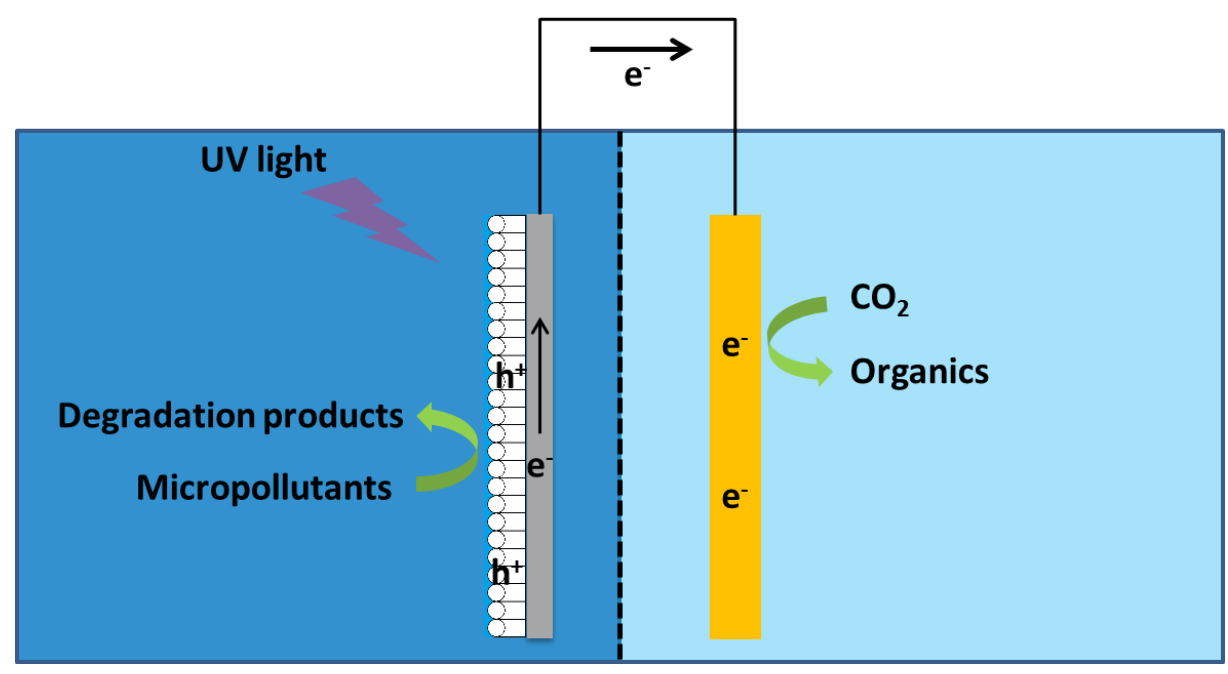

Figure 7. 4 Schematic representation of the concept of coupling micropollutant removal processes with resource in PFC system

\subsection{Concluding remarks}

To safe guard the aquatic environment and safe water supply, there is an urgent need for implementation of effective and economically feasible micropollutant removal technologies. This thesis contributes to a better understanding and further development of photochemical treatment processes for micropollutant removal from aqueous streams. The application of $\mathrm{TiO}_{2}$ nanotube array (TNA) as immobilized photocatalyst for micropollutant removal was studied, and the red light induced methylene blue photosensitization (MB/Red-light) as an alternative micropollutant removal technique was investigated. The 
results suggest that TNA is a promising immobilized photocatalyst. By applying it in photocatalytic fuel cell system, the removal of micropollutants can be further improved. The $M B /$ Red-light technique was found to be an effective alternative technique for degrading micropollutants in water. A big advantage of this method is the selectivity of the produced ROS. Additionally, the use of red light results in higher penetration depth in water with particles and dissolved molecules, generally the status of wastewater (effluents) in practice. Thus, effective micropollutant removal can be expected even in complex water matrices with high level of background water constituents. Nevertheless, research efforts are still needed for scaling up of the studied techniques, so in this Chapter, further research topics are suggested, namely: (1) long term stability of TNA; (2) by-products formation and toxicity; (3) limits of the MB/Red-light technique; (4) process optimization and reactor design. In addition, to improve the sustainability of micropollutant removal techniques, two possible directions are recommended: (1) use of solar light; (2) integrating micropollutant removal with resource (e.g. methane, methanol) recovery processes. 
Bibliography

\section{Bibliography}




\section{Bibliography}

[1] R.P. Schwarzenbach, B.I. Escher, K. Fenner, T.B. Hofstetter, C.A. Johnson, U. von Gunten, B. Wehrli, The Challenge of Micropollutants in Aquatic Systems, Science 313 (2006) 1072-1077.

[2] E. Oftadeh, M. Shourian, B. Saghafian, Evaluation of the Bankruptcy Approach for Water Resources Allocation Conflict Resolution at Basin Scale, Iran's Lake Urmia Experience, Water Resources Management 30 (2016) 3519-3533.

[3] L. Zhang, J. De Vrieze, T.L.G. Hendrickx, W. Wei, H. Temmink, H. Rijnaarts, G. Zeeman, Anaerobic treatment of raw domestic wastewater in a UASB-digester at $10^{\circ} \mathrm{C}$ and microbial community dynamics, Chemical Engineering Journal 334 (2018) 2088-2097.

[4] O.G. Gonzalo, I. Ruiz, M. Soto, Integrating pretreatment and denitrification in constructed wetland systems, Science of the total environment 584-585 (2017) 1300-1309.

[5] M. Sánchez-Polo, J. Rivera-Utrilla, G. Prados-Joya, R. Ocampo-Pérez, Metronidazole photodegradation in aqueous solution by using photosensitizers and hydrogen peroxide, Journal of Chemical Technology \& Biotechnology 87 (2012) 1202-1208.

[6] C. Martínez, M. Canle L, M.I. Fernández, J.A. Santaballa, J. Faria, Aqueous degradation of diclofenac by heterogeneous photocatalysis using nanostructured materials, Applied Catalysis B: Environmental 107 (2011) 110-118.

[7] A. Nikolaou, S. Meric, D. Fatta, Occurrence patterns of pharmaceuticals in water and wastewater environments, Analytical and bioanalytical chemistry 387 (2007) 1225-1234.

[8] C. Tixier, H.P. Singer, S. Oellers, S.R. Müller, Occurrence and fate of carbamazepine, clofibric acid, diclofenac, ibuprofen, ketoprofen, and naproxen in surface waters, Environmental science \& technology 37 (2003) 1061-1068. 
[9] R. Andreozzi, M. Raffaele, P. Nicklas, Pharmaceuticals in STP effluents and their solar photodegradation in aquatic environment, Chemosphere 50 (2003) 13191330.

[10] T.E. Doll, F.H. Frimmel, Fate of pharmaceuticals--photodegradation by simulated solar UV-light, Chemosphere 52 (2003) 1757-1769.

[11] T.A. Ternes, M. Meisenheimer, D. McDowell, F. Sacher, H.-J. Brauch, B. HaistGulde, G. Preuss, U. Wilme, N. Zulei-Seibert, Removal of pharmaceuticals during drinking water treatment, Environmental science \& technology 36 (2002) 38553863.

[12] T.E. Félix-Cañedo, J.C. Durán-Álvarez, B. Jiménez-Cisneros, The occurrence and distribution of a group of organic micropollutants in Mexico City's water sources, Science of the total environment 454-455 (2013) 109-118.

[13] A. Jurado, E. Vàzquez-Suñé, J. Carrera, M. López de Alda, E. Pujades, D. Barceló, Emerging organic contaminants in groundwater in Spain: A review of sources, recent occurrence and fate in a European context, Science of the total environment 440 (2012) 82-94.

[14] P. Schipper, M. Vissers, A.v.d. Linden, Pesticides in groundwater and drinking water wells: overview of the situation in the Netherlands, Water Science and Technology 57 (2008) 1277-1286.

[15] S.D. Richardson, T.A. Ternes, Water Analysis: Emerging Contaminants and Current Issues, Analytical Chemistry 86 (2014) 2813-2848.

[16] A. Pal, K.Y.-H. Gin, A.Y.-C. Lin, M. Reinhard, Impacts of emerging organic contaminants on freshwater resources: Review of recent occurrences, sources, fate and effects, Science of The Total Environment 408 (2010) 6062-6069.

[17] Y. Luo, W. Guo, H.H. Ngo, L.D. Nghiem, F.I. Hai, J. Zhang, S. Liang, X.C. Wang, A review on the occurrence of micropollutants in the aquatic environment and their fate and removal during wastewater treatment, Science of the total environment 473-474 (2014) 619-641. 


\section{Bibliography}

[18] M.O. Barbosa, N.F.F. Moreira, A.R. Ribeiro, M.F.R. Pereira, A.M.T. Silva, Occurrence and removal of organic micropollutants: An overview of the watch list of EU Decision 2015/495, Water Research 94 (2016) 257-279.

[19] P. Verlicchi, A. Galletti, M. Petrovic, D. Barceló, Hospital effluents as a source of emerging pollutants: an overview of micropollutants and sustainable treatment options, Journal of Hydrology 389 (2010) 416-428.

[20] B. Kasprzyk-Hordern, R.M. Dinsdale, A.J. Guwy, The removal of pharmaceuticals, personal care products, endocrine disruptors and illicit drugs during wastewater treatment and its impact on the quality of receiving waters, Water Research 43 (2009) 363-380.

[21] E. De Laurentiis, S. Chiron, S. Kouras-Hadef, C. Richard, M. Minella, V. Maurino, C. Minero, D. Vione, Photochemical Fate of Carbamazepine in Surface Freshwaters: Laboratory Measures and Modeling, Environmental science \& technology 46 (2012) 8164-8173.

[22] I. Ferrer, C.E. Heine, E.M. Thurman, Combination of LC/TOF-MS and LC/ion trap MS/MS for the identification of diphenhydramine in sediment samples, Analytical Chemistry 76 (2004) 1437-1444.

[23] J. Margot, L. Rossi, D.A. Barry, C. Holliger, A review of the fate of micropollutants in wastewater treatment plants, Wiley Interdisciplinary Reviews: Water 2 (2015) 457-487.

[24] D.J. Lapworth, N. Baran, M.E. Stuart, R.S. Ward, Emerging organic contaminants in groundwater: A review of sources, fate and occurrence, Environmental Pollution 163 (2012) 287-303.

[25] M.I. Farré, S. Pérez, L. Kantiani, D. Barceló, Fate and toxicity of emerging pollutants, their metabolites and transformation products in the aquatic environment, TrAC Trends in Analytical Chemistry 27 (2008) 991-1007. 
[26] M.D. Hernando, M. Mezcua, A.R. Fernández-Alba, D. Barceló, Environmental risk assessment of pharmaceutical residues in wastewater effluents, surface waters and sediments, Talanta 69 (2006) 334-342.

[27] P. Verlicchi, M. Al Aukidy, E. Zambello, Occurrence of pharmaceutical compounds in urban wastewater: Removal, mass load and environmental risk after a secondary treatment-A review, Science of The Total Environment 429 (2012) 123-155.

[28] C. Bredhult, B.-M. Bäcklin, M. Olovsson, Effects of some endocrine disruptors on the proliferation and viability of human endometrial endothelial cells in vitro, Reproductive Toxicology 23 (2007) 550-559.

[29] M. Helmestam, E. Davey, A. Stavreus-Evers, M. Olovsson, Bisphenol A affects human endometrial endothelial cell angiogenic activity in vitro, Reproductive Toxicology 46 (2014) 69-76.

[30] M. Forte, L. Mita, L. Cobellis, V. Merafina, R. Specchio, S. Rossi, D.G. Mita, L. Mosca, M.A. Castaldi, M. De Falco, V. Laforgia, S. Crispi, Triclosan and bisphenol a affect decidualization of human endometrial stromal cells, Molecular and Cellular Endocrinology 422 (2016) 74-83.

[31] S. Rodriguez-Mozaz, S. Chamorro, E. Marti, B. Huerta, M. Gros, A. SànchezMelsió, C.M. Borrego, D. Barceló, J.L. Balcázar, Occurrence of antibiotics and antibiotic resistance genes in hospital and urban wastewaters and their impact on the receiving river, Water Research 69 (2015) 234-242.

[32] D. Das, R.J. Cuthbert, R.D. Jakati, V. Prakash, Diclofenac is toxic to the Himalayan Vulture Gyps himalayensis, Bird Conservation International 21 (2011) 72-75.

[33] J.L. Oaks, M. Gilbert, M.Z. Virani, R.T. Watson, C.U. Meteyer, B.A. Rideout, H. Shivaprasad, S. Ahmed, M.J.I. Chaudhry, M. Arshad, Diclofenac residues as the cause of vulture population decline in Pakistan, Nature 427 (2004) 630. 


\section{Bibliography}

[34] P. Gao, M. Munir, I. Xagoraraki, Correlation of tetracycline and sulfonamide antibiotics with corresponding resistance genes and resistant bacteria in a conventional municipal wastewater treatment plant, Science of the total environment 421-422 (2012) 173-183.

[35] D. Calderón-Preciado, V. Matamoros, J.M. Bayona, Occurrence and potential crop uptake of emerging contaminants and related compounds in an agricultural irrigation network, Science of the total environment 412-413 (2011) 14-19.

[36] O.A. Jones, J.N. Lester, N. Voulvoulis, Pharmaceuticals: a threat to drinking water?, Trends in Biotechnology 23 (2005) 163-167.

[37] S. Webb, T. Ternes, M. Gibert, K. Olejniczak, Indirect human exposure to pharmaceuticals via drinking water, Toxicology Letters 142 (2003) 157-167.

[38] B.W. Schwab, E.P. Hayes, J.M. Fiori, F.J. Mastrocco, N.M. Roden, D. Cragin, R.D. Meyerhoff, V.J. D'Aco, P.D. Anderson, Human pharmaceuticals in US surface waters: A human health risk assessment, Regulatory Toxicology and Pharmacology 42 (2005) 296-312.

[39] A. Verliefde, E. Cornelissen, G. Amy, B. Van der Bruggen, H. van Dijk, Priority organic micropollutants in water sources in Flanders and the Netherlands and assessment of removal possibilities with nanofiltration, Environmental Pollution 146 (2007) 281-289.

[40] S. Kim, D.S. Aga, Potential Ecological and Human Health Impacts of Antibiotics and Antibiotic-Resistant Bacteria from Wastewater Treatment Plants, Journal of Toxicology and Environmental Health, Part B 10 (2007) 559-573.

[41] Q.T. Jiang, T.K.M. Lee, K. Chen, H.L. Wong, J.S. Zheng, J.P. Giesy, K.K.W. Lo, N. Yamashita, P.K.S. Lam, Human health risk assessment of organochlorines associated with fish consumption in a coastal city in China, Environmental Pollution 136 (2005) 155-165. 
[42] L. Cizmas, V.K. Sharma, C.M. Gray, T.J. McDonald, Pharmaceuticals and personal care products in waters: occurrence, toxicity, and risk, Environmental Chemistry Letters 13 (2015) 381-394.

[43] A.D. Correia, S. Freitas, M. Scholze, J.F. Goncalves, P. Booij, M.H. Lamoree, E. Mañanós, M.A. Reis-Henriques, Mixtures of Estrogenic Chemicals Enhance Vitellogenic Response in Sea Bass, Environmental Health Perspectives 115 (2007) $115-121$.

[44] J.V. Brian, C.A. Harris, M. Scholze, T. Backhaus, P. Booy, M. Lamoree, G. Pojana, N. Jonkers, T. Runnalls, A. Bonfà, A. Marcomini, J.P. Sumpter, Accurate Prediction of the Response of Freshwater Fish to a Mixture of Estrogenic Chemicals, Environmental Health Perspectives 113 (2005) 721-728.

[45] P.A. Neale, S. Ait-Aissa, W. Brack, N. Creusot, M.S. Denison, B. Deutschmann, K. Hilscherová, H. Hollert, M. Krauss, J. Novák, T. Schulze, T.-B. Seiler, H. Serra, Y. Shao, B.I. Escher, Linking in Vitro Effects and Detected Organic Micropollutants in Surface Water Using Mixture-Toxicity Modeling, Environmental science \& technology 49 (2015) 14614-14624.

[46] J.Y.M. Tang, S. McCarty, E. Glenn, P.A. Neale, M.S.J. Warne, B.I. Escher, Mixture effects of organic micropollutants present in water: Towards the development of effect-based water quality trigger values for baseline toxicity, Water Research 47 (2013) 3300-3314.

[47] C.o.t.E. Union, Directive 2013/39/EU of the European Parliament and of the Council of 12 August 2013 amending Directives 2000/60/EC and 2008/105/EC as regards priority substances in the field of water policy Text with EEA relevance, in: E. Parliament (Ed.), 2013.

[48] W. Liu, N.B. Sutton, H.H.M. Rijnaarts, A.A.M. Langenhoff, Pharmaceutical removal from water with iron- or manganese-based technologies: A review, Critical Reviews in Environmental Science and Technology 46 (2016) 1584-1621. 


\section{Bibliography}

[49] A.T. Besha, A.Y. Gebreyohannes, R.A. Tufa, D.N. Bekele, E. Curcio, L. Giorno, Removal of emerging micropollutants by activated sludge process and membrane bioreactors and the effects of micropollutants on membrane fouling: A review, Journal of Environmental Chemical Engineering 5 (2017) 2395-2414.

[50] A.Y.C. Tong, R. Braund, D.S. Warren, B.M. Peake, TiO2-assisted photodegradation of pharmaceuticals - a review, Cent Eur J Chem 10 (2012) 9891027.

[51] Y. He, Removal of pharmaceutically active compounds in constructed wetlands: mechanisms and application, Wageningen University, Wageningen, 2017.

[52] W. Liu, Anaerobic manganese- or iron-mediated pharmaceutical degradation in water, Wageningen University, Wageningen, 2018.

[53] H.A. de Wilt, Pharmaceutical removal: synergy between biological and chemical processes for wastewater treatment, Wageningen University, Wageningen, 2018.

[54] L. Kovalova, H. Siegrist, U. von Gunten, J. Eugster, M. Hagenbuch, A. Wittmer, R. Moser, C.S. McArdell, Elimination of Micropollutants during Post-Treatment of Hospital Wastewater with Powdered Activated Carbon, Ozone, and UV, Environmental Science \& Technology 47 (2013) 7899-7908.

[55] D. Vogna, R. Marotta, A. Napolitano, R. Andreozzi, M. d'Ischia, Advanced oxidation of the pharmaceutical drug diclofenac with UV/H2O2 and ozone, Water Research 38 (2004) 414-422.

[56] J.C. Kruithof, P.C. Kamp, B.J. Martijn, UV/H2O2Treatment: A Practical Solution for Organic Contaminant Control and Primary Disinfection, Ozone: Science \& Engineering 29 (2007) 273-280.

[57] I. Kim, N. Yamashita, H. Tanaka, Performance of UV and UV/H2O2 processes for the removal of pharmaceuticals detected in secondary effluent of a sewage treatment plant in Japan, Journal of Hazardous Materials 166 (2009) 1134-1140. 
[58] I. Kim, N. Yamashita, H. Tanaka, Photodegradation of pharmaceuticals and personal care products during UV and UV/H2O2 treatments, Chemosphere 77 (2009) 518-525.

[59] F. Yuan, C. Hu, X. Hu, J. Qu, M. Yang, Degradation of selected pharmaceuticals in aqueous solution with UV and UV/H2O2, Water Research 43 (2009) 1766-1774. [60] J.Q. Chen, Z.J. Hu, N.X. Wang, Photocatalytic mineralization of glyphosate in a small-scale plug flow simulation reactor by UV/TiO2, J Environ Sci Heal B 47 (2012) 579-588.

[61] P. Calza, V. Sakkas, C. Medana, C. Baiocchi, A. Dimou, E. Pelizzetti, T. Albanis, Photocatalytic degradation study of diclofenac over aqueous TiO2 suspensions, Applied Catalysis B: Environmental 67 (2006) 197-205.

[62] J. Kuipers, Distributed light sources for photocatalytic water treatment, Wageningen University, Wageningen, 2014.

[63] L.A. Pérez-Estrada, S. Malato, W. Gernjak, A. Agüera, E.M. Thurman, I. Ferrer, A.R. Fernández-Alba, Photo-Fenton Degradation of Diclofenac: Identification of Main Intermediates and Degradation Pathway, Environmental science \& technology 39 (2005) 8300-8306.

[64] E. Díez-Mato, F.C. Cortezón-Tamarit, S. Bogialli, D. García-Fresnadillo, M.D. Marazuela, Phototransformation of model micropollutants in water samples by photocatalytic singlet oxygen production in heterogeneous medium, Applied Catalysis B: Environmental 160-161 (2014) 445-455.

[65] Y. Ye, H. Bruning, D. Yntema, M. Mayer, H. Rijnaarts, Homogeneous photosensitized degradation of pharmaceuticals by using red light LED as light source and methylene blue as photosensitizer, Chemical Engineering Journal 316 (2017) 872-881.

[66] X. Jin, S. Peldszus, P.M. Huck, Reaction kinetics of selected micropollutants in ozonation and advanced oxidation processes, Water Research 46 (2012) 6519-6530. 


\section{Bibliography}

[67] B.A. Wols, C.H.M. Hofman-Caris, D.J.H. Harmsen, E.F. Beerendonk, Degradation of 40 selected pharmaceuticals by UV/H2O2, Water Research 47 (2013) 5876-5888.

[68] L.L.S. Silva, J.C.S. Sales, J.C. Campos, D.M. Bila, F.V. Fonseca, Advanced oxidative processes and membrane separation for micropollutant removal from biotreated domestic wastewater, Environmental Science and Pollution Research 24 (2017) 6329-6338.

[69] Z. Shu, A. Singh, N. Klamerth, K. McPhedran, J.R. Bolton, M. Belosevic, M. Gamal El-Din, Pilot-scale UV/H2O2 advanced oxidation process for municipal reuse water: Assessing micropollutant degradation and estrogenic impacts on goldfish (Carassius auratus L.), Water Research 101 (2016) 157-166.

[70] S. Semitsoglou-Tsiapou, M.R. Templeton, N.J. Graham, L.H. Leal, B.J. Martijn, A. Royce, J.C. Kruithof, Low pressure UV/H 202 treatment for the degradation of the pesticides metaldehyde, clopyralid and mecoprop-Kinetics and reaction product formation, Water Research 91 (2016) 285-294.

[71] X. Ma, C. Zhang, J. Deng, Y. Song, Q. Li, Y. Guo, C. Li, Simultaneous Degradation of Estrone, $17 \beta$-Estradiol and $17 \alpha$-Ethinyl Estradiol in an Aqueous UV/H2O2 System, International Journal of Environmental Research and Public Health 12 (2015) 12016. [72] S. Giannakis, F.A. Gamarra Vives, D. Grandjean, A. Magnet, L.F. De Alencastro, C. Pulgarin, Effect of advanced oxidation processes on the micropollutants and the effluent organic matter contained in municipal wastewater previously treated by three different secondary methods, Water Research 84 (2015) 295-306.

[73] J.C. Kruithof, P.C. Kamp, B.J. Martijn, UV/H2O2 Treatment: A Practical Solution for Organic Contaminant Control and Primary Disinfection, Ozone: Science \& Engineering 29 (2007) 273-280.

[74] N.M. Vieno, H. Härkki, T. Tuhkanen, L. Kronberg, Occurrence of pharmaceuticals in river water and their elimination in a pilot-scale drinking water treatment plant, Environmental science \& technology 41 (2007) 5077-5084. 
[75] R. Andreozzi, V. Caprio, A. Insola, R. Marotta, Advanced oxidation processes (AOP) for water purification and recovery, Catal Today 53 (1999) 51-59.

[76] Y. Valcárcel, F. Martínez, S. González-Alonso, Y. Segura, M. Catalá, R. Molina, J.C. Montero-Rubio, N. Mastroianni, M. López de Alda, C. Postigo, D. Barceló, Drugs of abuse in surface and tap waters of the Tagus River basin: Heterogeneous photoFenton process is effective in their degradation, Environment International 41 (2012) 35-43.

[77] A.R. Ribeiro, O.C. Nunes, M.F.R. Pereira, A.M.T. Silva, An overview on the advanced oxidation processes applied for the treatment of water pollutants defined in the recently launched Directive 2013/39/EU, Environment International 75 (2015) 33-51.

[78] I. Carra, J.L. García Sánchez, J.L. Casas López, S. Malato, J.A. Sánchez Pérez, Phenomenological study and application of the combined influence of iron concentration and irradiance on the photo-Fenton process to remove micropollutants, Science of the total environment 478 (2014) 123-132.

[79] I. Carra, J.A. Sánchez Pérez, S. Malato, O. Autin, B. Jefferson, P. Jarvis, Application of high intensity UVC-LED for the removal of acetamiprid with the photo-Fenton process, Chemical Engineering Journal 264 (2015) 690-696.

[80] I.N. Dias, B.S. Souza, J.H.O.S. Pereira, F.C. Moreira, M. Dezotti, R.A.R. Boaventura, V.J.P. Vilar, Enhancement of the photo-Fenton reaction at near neutral $\mathrm{pH}$ through the use of ferrioxalate complexes: A case study on trimethoprim and sulfamethoxazole antibiotics removal from aqueous solutions, Chemical Engineering Journal 247 (2014) 302-313.

[81] M.J. Lima, C.G. Silva, A.M.T. Silva, J.C.B. Lopes, M.M. Dias, J.L. Faria, Homogeneous and heterogeneous photo-Fenton degradation of antibiotics using an innovative static mixer photoreactor, Chemical Engineering Journal 310 (2017) 342-351. 


\section{Bibliography}

[82] N. López, S. Plaza, A. Afkhami, P. Marco, J. Giménez, S. Esplugas, Treatment of Diphenhydramine with different AOPs including photo-Fenton at circumneutral $\mathrm{pH}$, Chemical Engineering Journal 318 (2017) 112-120.

[83] J.J. Pignatello, E. Oliveros, A. MacKay, Advanced Oxidation Processes for Organic Contaminant Destruction Based on the Fenton Reaction and Related Chemistry, Critical Reviews in Environmental Science and Technology 36 (2006) 184.

[84] N. Klamerth, S. Malato, A. Agüera, A. Fernández-Alba, G. Mailhot, Treatment of Municipal Wastewater Treatment Plant Effluents with Modified Photo-Fenton As a Tertiary Treatment for the Degradation of Micro Pollutants and Disinfection, Environmental science \& technology 46 (2012) 2885-2892.

[85] M. Umar, H.A. Aziz, M.S. Yusoff, Trends in the use of Fenton, electro-Fenton and photo-Fenton for the treatment of landfill leachate, Waste Management 30 (2010) 2113-2121.

[86] R.P. Schwarzenbach, P.M. Gschwend, D.M. Imboden, Direct Photolysis, Environmental Organic Chemistry, John Wiley \& Sons, Inc.2005, pp. 611-654.

[87] R.P. Schwarzenbach, P.M. Gschwend, D.M. Imboden, Indirect Photolysis: Reactions with Photooxidants in Natural Waters and in the Atmosphere, Environmental Organic Chemistry, John Wiley \& Sons, Inc.2005, pp. 655-686.

[88] K. Song, M. Mohseni, F. Taghipour, Application of ultraviolet light-emitting diodes (UV-LEDs) for water disinfection: A review, Water Research 94 (2016) 341349.

[89] H.Y. Kim, T.-H. Kim, S. Yu, Photolytic degradation of sulfamethoxazole and trimethoprim using UV-A, UV-C and vacuum-UV (VUV), Journal of Environmental Science and Health, Part A 50 (2015) 292-300.

[90] D. Fatta-Kassinos, M.I. Vasquez, K. Kümmerer, Transformation products of pharmaceuticals in surface waters and wastewater formed during photolysis and 
advanced oxidation processes - Degradation, elucidation of byproducts and assessment of their biological potency, Chemosphere 85 (2011) 693-709.

[91] O.S. Keen, E.M. Thurman, I. Ferrer, A.D. Dotson, K.G. Linden, Dimer formation during UV photolysis of diclofenac, Chemosphere 93 (2013) 1948-1956.

[92] S. Sanches, A. Penetra, A. Rodrigues, V.V. Cardoso, E. Ferreira, M.J. Benoliel, M.T. Barreto Crespo, J.G. Crespo, V.J. Pereira, Removal of pesticides from water combining low pressure UV photolysis with nanofiltration, Separation and purification Technology 115 (2013) 73-82.

[93] L. Paredes, F. Omil, J.M. Lema, M. Carballa, What happens with organic micropollutants during UV disinfection in WWTPs? A global perspective from laboratory to full-scale, Journal of Hazardous Materials 342 (2018) 670-678.

[94] W. Yang, H. Zhou, N. Cicek, Treatment of Organic Micropollutants in Water and Wastewater by UV-Based Processes: A Literature Review, Critical Reviews in Environmental Science and Technology 44 (2014) 1443-1476.

[95] A. Fujishima, K. Honda, Electrochemical Photolysis of Water at a Semiconductor Electrode, Nature 238 (1972) 37.

[96] N. Daneshvar, S. Aber, M.S. Dorraji, A. Khataee, M. Rasoulifard, Photocatalytic degradation of the insecticide diazinon in the presence of prepared nanocrystalline ZnO powders under irradiation of UV-C light, Separation and purification Technology 58 (2007) 91-98.

[97] J. Han, Y. Liu, N. Singhal, L. Wang, W. Gao, Comparative photocatalytic degradation of estrone in water by $\mathrm{ZnO}$ and $\mathrm{TiO} 2$ under artificial UVA and solar irradiation, Chemical Engineering Journal 213 (2012) 150-162.

[98] C. Martínez, M. Canle L, M.I. Fernández, J.A. Santaballa, J. Faria, Kinetics and mechanism of aqueous degradation of carbamazepine by heterogeneous photocatalysis using nanocrystalline $\mathrm{TiO} 2, \mathrm{ZnO}$ and multi-walled carbon nanotubes-anatase composites, Applied Catalysis B: Environmental 102 (2011) 563-571. 


\section{Bibliography}

[99] E. Regulska, D.M. Brus, P. Rodziewicz, S. Sawicka, J. Karpinska, Photocatalytic degradation of hazardous Food Yellow 13 in $\mathrm{TiO} 2$ and $\mathrm{ZnO}$ aqueous and river water suspensions, Catal Today 266 (2016) 72-81.

[100] A. Rey, P. García-Muñoz, M.D. Hernández-Alonso, E. Mena, S. GarcíaRodríguez, F.J. Beltrán, WO3-TiO2 based catalysts for the simulated solar radiation assisted photocatalytic ozonation of emerging contaminants in a municipal wastewater treatment plant effluent, Applied Catalysis B: Environmental 154 (2014) 274-284.

[101] Z. Liu, X. Zhang, S. Nishimoto, T. Murakami, A. Fujishima, Efficient photocatalytic degradation of gaseous acetaldehyde by highly ordered TiO2 nanotube arrays, Environmental science \& technology 42 (2008) 8547-8551.

[102] L.A. Ioannou, E. Hapeshi, M.I. Vasquez, D. Mantzavinos, D. Fatta-Kassinos, Solar/TiO2 photocatalytic decomposition of $\beta$-blockers atenolol and propranolol in water and wastewater, Solar Energy 85 (2011) 1915-1926.

[103] R.P. Cavalcante, R.F. Dantas, B. Bayarri, O. González, J. Giménez, S. Esplugas, A. Machulek Junior, Synthesis and characterization of B-doped TiO2 and their performance for the degradation of metoprolol, Catal Today 252 (2015) 27-34.

[104] R.P. Cavalcante, R.F. Dantas, B. Bayarri, O. González, J. Giménez, S. Esplugas, A. Machulek Junior, Photocatalytic mechanism of metoprolol oxidation by photocatalysts $\mathrm{TiO} 2$ and $\mathrm{TiO} 2$ doped with $5 \% \mathrm{~B}$ : Primary active species and intermediates, Applied Catalysis B: Environmental 194 (2016) 111-122.

[105] C.S. Chiou, J.L. Shie, C.Y. Chang, C.C. Liu, C.T. Chang, Degradation of di-n-butyl phthalate using photoreactor packed with $\mathrm{TiO} 2 \mathrm{immobilized} \mathrm{on} \mathrm{glass} \mathrm{beads,} \mathrm{Journal}$ of Hazardous Materials 137 (2006) 1123-1129.

[106] M.H. Habibi, A. Hassanzadeh, S. Mahdavi, The effect of operational parameters on the photocatalytic degradation of three textile azo dyes in aqueous TiO2 suspensions, Journal of Photochemistry and Photobiology A: Chemistry 172 (2005) 89-96. 
[107] A. Achilleos, E. Hapeshi, N.P. Xekoukoulotakis, D. Mantzavinos, D. FattaKassinos, Factors affecting diclofenac decomposition in water by UV-A/TiO2 photocatalysis, Chemical Engineering Journal 161 (2010) 53-59.

[108] M. Canle L, J.A. Santaballa, E. Vulliet, On the mechanism of TiO2photocatalyzed degradation of aniline derivatives, Journal of Photochemistry and Photobiology A: Chemistry 175 (2005) 192-200.

[109] J. Chen, D.F. Ollis, W.H. Rulkens, H. Bruning, Photocatalyzed oxidation of alcohols and organochlorides in the presence of native $\mathrm{TiO} 2$ and metallized $\mathrm{TiO} 2$ suspensions. Part (I): Photocatalytic activity and pH influence, Water Research 33 (1999) 661-668.

[110] H. Choi, S.R. Al-Abed, D.D. Dionysiou, E. Stathatos, P. Lianos, Chapter 8 TiO2Based Advanced Oxidation Nanotechnologies for Water Purification and Reuse, in: I.C. Escobar, A.I. Schäfer (Eds.) Sustainability Science and Engineering, Elsevier2010, pp. 229-254.

[111] D.D. Dionysiou, A.P. Khodadoust, A.M. Kern, M.T. Suidan, I. Baudin, J.-M. Laîné, Continuous-mode photocatalytic degradation of chlorinated phenols and pesticides in water using a bench-scale TiO2 rotating disk reactor, Applied Catalysis B: Environmental 24 (2000) 139-155.

[112] T. Fotiou, T.M. Triantis, T. Kaloudis, K.E. O'Shea, D.D. Dionysiou, A. Hiskia, Assessment of the roles of reactive oxygen species in the UV and visible light photocatalytic degradation of cyanotoxins and water taste and odor compounds using C-TiO2, Water Research 90 (2016) 52-61.

[113] O. Sacco, V. Vaiano, C. Han, D. Sannino, D.D. Dionysiou, Photocatalytic removal of atrazine using $\mathrm{N}$-doped TiO2 supported on phosphors, Applied Catalysis B: Environmental 164 (2015) 462-474.

[114] S. Zheng, W. Jiang, Y. Cai, D.D. Dionysiou, K.E. O'Shea, Adsorption and photocatalytic degradation of aromatic organoarsenic compounds in $\mathrm{TiO} 2$ suspension, Catal Today 224 (2014) 83-88. 


\section{Bibliography}

[115] E.O. Oseghe, P.G. Ndungu, S.B. Jonnalagadda, Photocatalytic degradation of 4-chloro-2-methylphenoxyacetic acid using W-doped TiO2, Journal of Photochemistry and Photobiology A: Chemistry 312 (2015) 96-106.

[116] A. Topalov, B. Abramović, D. Molnár-Gábor, J. Csanádi, O. Arcson, Photocatalytic oxidation of the herbicide (4-chloro-2-methylphenoxy)acetic acid (MCPA) over TiO2, Journal of Photochemistry and Photobiology A: Chemistry 140 (2001) 249-253.

[117] A. Zertal, D. Molnár-Gábor, M.A. Malouki, T. Sehili, P. Boule, Photocatalytic transformation of 4-chloro-2-methylphenoxyacetic acid (MCPA) on several kinds of TiO2, Applied Catalysis B: Environmental 49 (2004) 83-89.

[118] D.V. Šojić, V.N. Despotović, N.D. Abazović, M.I. Čomor, B.F. Abramović, Photocatalytic degradation of selected herbicides in aqueous suspensions of doped titania under visible light irradiation, Journal of Hazardous Materials 179 (2010) 4956.

[119] R.P. Cavalcante, R.F. Dantas, H. Wender, B. Bayarri, O. González, J. Giménez, S. Esplugas, A. Machulek Jr, Photocatalytic treatment of metoprolol with B-doped TiO2: Effect of water matrix, toxicological evaluation and identification of intermediates, Applied Catalysis B: Environmental 176-177 (2015) 173-182.

[120] K. Djebbar, A. Zertal, T. Sehili, Photocatalytic Degradation of 2,4Dichlorophenoxyacetic Acid and 4-Chloro-2-Methylphenoxyacetic Acid in Water by using TiO2, Environmental Technology 27 (2006) 1191-1197.

[121] M.J. Benotti, B.D. Stanford, E.C. Wert, S.A. Snyder, Evaluation of a photocatalytic reactor membrane pilot system for the removal of pharmaceuticals and endocrine disrupting compounds from water, Water Research 43 (2009) 15131522.

[122] Y. He, N.B. Sutton, H.H.H. Rijnaarts, A.A.M. Langenhoff, Degradation of pharmaceuticals in wastewater using immobilized $\mathrm{TiO} 2$ photocatalysis under simulated solar irradiation, Applied Catalysis B: Environmental 182 (2016) 132-141. 
[123] J. Fenoll, P. Sabater, G. Navarro, G. Pérez-Lucas, S. Navarro, Photocatalytic transformation of sixteen substituted phenylurea herbicides in aqueous semiconductor suspensions: Intermediates and degradation pathways, Journal of Hazardous Materials 244-245 (2013) 370-379.

[124] S. Garcia-Segura, E. Brillas, Applied photoelectrocatalysis on the degradation of organic pollutants in wastewaters, Journal of Photochemistry and Photobiology C: Photochemistry Reviews 31 (2017) 1-35.

[125] G.G. Bessegato, J.C. Cardoso, M.V.B. Zanoni, Enhanced photoelectrocatalytic degradation of an acid dye with boron-doped TiO 2 nanotube anodes, Catal Today 240 (2015) 100-106.

[126] T.E.o.E. Britannica, Photosensitization, Encyclopædia Britannica, inc., 2017.

[127] M.C. DeRosa, R.J. Crutchley, Photosensitized singlet oxygen and its applications, Coordination Chemistry Reviews 233 (2002) 351-371.

[128] I.E. Kochevar, R.W. Redmond, [2] Photosensitized production of singlet oxygen, Methods in Enzymology, Academic Press2000, pp. 20-28.

[129] J. Lee, S. Hong, Y. Mackeyev, C. Lee, E. Chung, L.J. Wilson, J.-H. Kim, P.J. Alvarez, Photosensitized oxidation of emerging organic pollutants by tetrakis C60 aminofullerene-derivatized silica under visible light irradiation, Environmental science \& technology 45 (2011) 10598-10604.

[130] H. Kim, W. Kim, Y. Mackeyev, G.-S. Lee, H.-J. Kim, T. Tachikawa, S. Hong, S. Lee, J. Kim, L.J. Wilson, T. Majima, P.J.J. Alvarez, W. Choi, J. Lee, Selective Oxidative Degradation of Organic Pollutants by Singlet Oxygen-Mediated Photosensitization: Tin Porphyrin versus C60 Aminofullerene Systems, Environmental science \& technology 46 (2012) 9606-9613.

[131] M. Gmurek, M. Foszpańczyk, M. Olak-Kucharczyk, D. Gryglik, S. Ledakowicz, Photosensitive chitosan for visible-light water pollutant degradation, Chemical Engineering Journal 318 (2017) 240-246. 


\section{Bibliography}

[132] W.-L. Wang, Q.-Y. Wu, N. Huang, T. Wang, H.-Y. Hu, Synergistic effect between

UV and chlorine (UV/chlorine) on the degradation of carbamazepine: Influence factors and radical species, Water Research 98 (2016) 190-198.

[133] W.-L. Wang, Q.-Y. Wu, Z.-M. Li, Y. Lu, Y. Du, T. Wang, N. Huang, H.-Y. Hu, Lightemitting diodes as an emerging UV source for UV/chlorine oxidation: Carbamazepine degradation and toxicity changes, Chemical Engineering Journal 310 (2017) 148-156.

[134] X. Kong, J. Jiang, J. Ma, Y. Yang, W. Liu, Y. Liu, Degradation of atrazine by UV/chlorine: Efficiency, influencing factors, and products, Water Research 90 (2016) 15-23.

[135] I. Kim, H. Tanaka, Use of ozone-based processes for the removal of pharmaceuticals detected in a wastewater treatment plant, Water Environment Research 82 (2010) 294-301.

[136] S. Irmak, O. Erbatur, A. Akgerman, Degradation of $17 \beta$-estradiol and bisphenol $\mathrm{A}$ in aqueous medium by using ozone and ozone/UV techniques, Journal of Hazardous Materials 126 (2005) 54-62.

[137] I. Kim, H. Tanaka, Energy consumption for PPCPs removal by $\mathrm{O} 3$ and O3/UV, Ozone: Science \& Engineering 33 (2011) 150-157.

[138] C. Sichel, C. Garcia, K. Andre, Feasibility studies: UV/chlorine advanced oxidation treatment for the removal of emerging contaminants, Water Research 45 (2011) 6371-6380.

[139] Y. Yang, X. Lu, J. Jiang, J. Ma, G. Liu, Y. Cao, W. Liu, J. Li, S. Pang, X. Kong, C. Luo, Degradation of sulfamethoxazole by UV, UV/H2O2 and UV/persulfate (PDS): Formation of oxidation products and effect of bicarbonate, Water Research 118 (2017) 196-207.

[140] H. Dong, Z. Qiang, J. Hu, J. Qu, Degradation of chloramphenicol by UV/chlorine treatment: Kinetics, mechanism and enhanced formation of halonitromethanes, Water Research 121 (2017) 178-185. 
[141] B.F. Abramović, D.V. Šojić, V.B. Anderluh, N.D. Abazović, M.I. Čomor, Nitrogen-doped TiO2 suspensions in photocatalytic degradation of mecoprop and (4-chloro-2-methylphenoxy)acetic acid herbicides using various light sources, Desalination 244 (2009) 293-302.

[142] M. Long, J. Brame, F. Qin, J. Bao, Q. Li, P.J.J. Alvarez, Phosphate Changes Effect of Humic Acids on TiO2 Photocatalysis: From Inhibition to Mitigation of ElectronHole Recombination, Environmental science \& technology 51 (2017) 514-521.

[143] H. Peng, Y. Chen, L. Mao, X. Zhang, Significant changes in the photo-reactivity of TiO2 in the presence of a capped natural dissolved organic matter layer, Water Research 110 (2017) 233-240.

[144] O. Autin, J. Hart, P. Jarvis, J. MacAdam, S.A. Parsons, B. Jefferson, The impact of background organic matter and alkalinity on the degradation of the pesticide metaldehyde by two advanced oxidation processes: UV/H2O2 and UV/TiO2, Water Research 47 (2013) 2041-2049.

[145] M. Dijkstra, A. Michorius, H. Buwalda, H. Panneman, J. Winkelman, A. Beenackers, Comparison of the efficiency of immobilized and suspended systems in photocatalytic degradation, Catal Today 66 (2001) 487-494.

[146] M. Dijkstra, H. Panneman, J. Winkelman, J. Kelly, A. Beenackers, Modeling the photocatalytic degradation of formic acid in a reactor with immobilized catalyst, Chemical Engineering Science 57 (2002) 4895-4907.

[147] G. Mascolo, R. Comparelli, M.L. Curri, G. Lovecchio, A. Lopez, A. Agostiano, Photocatalytic degradation of methyl red by TiO2: Comparison of the efficiency of immobilized nanoparticles versus conventional suspended catalyst, Journal of Hazardous Materials 142 (2007) 130-137.

[148] M.J. Arlos, M.M. Hatat-Fraile, R. Liang, L.M. Bragg, N.Y. Zhou, S.A. Andrews, M.R. Servos, Photocatalytic decomposition of organic micropollutants using immobilized TiO2 having different isoelectric points, Water Research 101 (2016) 351-361. 


\section{Bibliography}

[149] S. Murgolo, V. Yargeau, R. Gerbasi, F. Visentin, N. El Habra, G. Ricco, I. Lacchetti, M. Carere, M.L. Curri, G. Mascolo, A new supported TiO2 film deposited on stainless steel for the photocatalytic degradation of contaminants of emerging concern, Chemical Engineering Journal 318 (2017) 103-111.

[150] A. Manassero, M.L. Satuf, O.M. Alfano, Photocatalytic degradation of an emerging pollutant by TiO2-coated glass rings: a kinetic study, Environmental Science and Pollution Research 24 (2017) 6031-6039.

[151] M.H. Alhaji, K. Sanaullah, A. Khan, A. Hamza, A. Muhammad, M.S. Ishola, A.R.H. Rigit, S.A. Bhawani, Recent developments in immobilizing titanium dioxide on supports for degradation of organic pollutants in wastewater- A review, International Journal of Environmental Science and Technology 14 (2017) 20392052.

[152] V. Zwilling, E. Darque - Ceretti, A. Boutry - Forveille, D. David, M.-Y. Perrin, M. Aucouturier, Structure and physicochemistry of anodic oxide films on titanium and TA6V alloy, Surface and Interface Analysis 27 (1999) 629-637.

[153] V. Zwilling, M. Aucouturier, E. Darque-Ceretti, Anodic oxidation of titanium and TA6V alloy in chromic media. An electrochemical approach, Electrochimica Acta 45 (1999) 921-929.

[154] S.P. Albu, A. Ghicov, J.M. Macak, P. Schmuki, $250 \mu \mathrm{m}$ long anodic TiO2 nanotubes with hexagonal self - ordering, physica status solidi (RRL)-Rapid Research Letters 1 (2007).

[155] R. Beranek, H. Hildebrand, P. Schmuki, Self-organized porous titanium oxide prepared in $\mathrm{H} 2 \mathrm{SO}$ 4/HF electrolytes, Electrochemical and solid-state letters 6 (2003) B12-B14.

[156] J.M. Macak, H. Tsuchiya, L. Taveira, S. Aldabergerova, P. Schmuki, Smooth anodic TiO2 nanotubes, Angewandte Chemie International Edition 44 (2005) 74637465. 
[157] Z. Ma, J. Gao, X. Wu, Y. Xie, H. Yuan, Y. Shi, Preparation of Well-Aligned TiO2 Nanotubes with High Length-Diameter Aspect Ratio by Anodic Oxidation Method, Journal of nanoscience and nanotechnology 18 (2018) 5810-5816.

[158] S. Ozkan, A. Mazare, P. Schmuki, Critical parameters and factors in the formation of spaced TiO2 nanotubes by self-organizing anodization, Electrochimica Acta 268 (2018) 435-447.

[159] P. Roy, S. Berger, P. Schmuki, TiO2 nanotubes: synthesis and applications, Angewandte Chemie International Edition 50 (2011) 2904-2939.

[160] J. Macak, H. Tsuchiya, A. Ghicov, K. Yasuda, R. Hahn, S. Bauer, P. Schmuki, TiO 2 nanotubes: self-organized electrochemical formation, properties and applications, Current Opinion in Solid State and Materials Science 11 (2007) 3-18.

[161] C.B. Marien, T. Cottineau, D. Robert, P. Drogui, TiO2 Nanotube arrays: Influence of tube length on the photocatalytic degradation of Paraquat, Applied Catalysis B: Environmental 194 (2016) 1-6.

[162] Y.R. Smith, A. Kar, V. Subramanian, Investigation of physicochemical parameters that influence photocatalytic degradation of methyl orange over TiO2 nanotubes, Industrial \& Engineering Chemistry Research 48 (2009) 10268-10276. [163] J.M. Macak, M. Zlamal, J. Krysa, P. Schmuki, Self - Organized TiO2 Nanotube Layers as Highly Efficient Photocatalysts, small 3 (2007) 300-304.

[164] T.S. Natarajan, K. Natarajan, H.C. Bajaj, R.J. Tayade, Energy Efficient UV-LED Source and TiO2 Nanotube Array-Based Reactor for Photocatalytic Application, Industrial \& Engineering Chemistry Research 50 (2011) 7753-7762.

[165] M.K. Arfanis, P. Adamou, N.G. Moustakas, T.M. Triantis, A.G. Kontos, P. Falaras, Photocatalytic degradation of salicylic acid and caffeine emerging contaminants using titania nanotubes, Chemical Engineering Journal 310 (2017) 525-536. 


\section{Bibliography}

[166] M.N. Chong, B. Jin, C.W.K. Chow, C. Saint, Recent developments in photocatalytic water treatment technology: A review, Water Research 44 (2010) 2997-3027.

[167] N. Rioja, S. Zorita, F.J. Peñas, Effect of water matrix on photocatalytic degradation and general kinetic modeling, Applied Catalysis B: Environmental 180 (2016) 330-335.

[168] A.-G. Rincón, C. Pulgarin, Effect of pH, inorganic ions, organic matter and $\mathrm{H} 2 \mathrm{O} 2$ on E. coli K12 photocatalytic inactivation by TiO2: Implications in solar water disinfection, Applied Catalysis B: Environmental 51 (2004) 283-302.

[169] J. Zhu, S. Wang, J. Wang, D. Zhang, H. Li, Highly active and durable Bi2O3/TiO2 visible photocatalyst in flower-like spheres with surface-enriched Bi2O3 quantum dots, Applied Catalysis B: Environmental 102 (2011) 120-125.

[170] X. Yao, L. Chen, M. Liu, D. Feng, C. Wang, F. Lu, W. Wang, X. Wang, Y. Cheng, H. Liu, H. Chen, W. Wang, Rational design of Si/TiO2 heterojunction photocatalysts: Transfer matrix method, Applied Catalysis B: Environmental 221 (2018) 70-76.

[171] S. Xie, K. Ouyang, Degradation of refractory organic compounds by photocatalytic fuel cell with solar responsive WO3/FTO photoanode and airbreathing cathode, Journal of Colloid and Interface Science 500 (2017) 220-227.

[172] Y. Liu, J. Li, B. Zhou, X. Li, H. Chen, Q. Chen, Z. Wang, L. Li, J. Wang, W. Cai, Efficient electricity production and simultaneously wastewater treatment via a high-performance photocatalytic fuel cell, Water Research 45 (2011) 3991-3998.

[173] K. Li, H. Zhang, T. Tang, Y. Xu, D. Ying, Y. Wang, J. Jia, Optimization and application of TiO2/Ti-Pt photo fuel cell (PFC) to effectively generate electricity and degrade organic pollutants simultaneously, Water Research 62 (2014) 1-10.

[174] O. Autin, J. Hart, P. Jarvis, J. MacAdam, S.A. Parsons, B. Jefferson, The impact of background organic matter and alkalinity on the degradation of the pesticide metaldehyde by two advanced oxidation processes: UV/H2O2 and UV/TiO2, Water Research 47 (2013) 2041-2049. 
[175] O. Autin, J. Hart, P. Jarvis, J. MacAdam, S.A. Parsons, B. Jefferson, Comparison of UV/TiO2 and UV/H2O2 processes in an annular photoreactor for removal of micropollutants: Influence of water parameters on metaldehyde removal, quantum yields and energy consumption, Applied Catalysis B: Environmental 138 (2013) 268275.

[176] I. Carra, J.A. Sánchez Pérez, S. Malato, O. Autin, B. Jefferson, P. Jarvis, Performance of different advanced oxidation processes for tertiary wastewater treatment to remove the pesticide acetamiprid, Journal of Chemical Technology \& Biotechnology 91 (2016) 72-81.

[177] J. Brame, M. Long, Q. Li, P. Alvarez, Trading oxidation power for efficiency: Differential inhibition of photo-generated hydroxyl radicals versus singlet oxygen, Water Research 60 (2014) 259-266.

[178] Y.-P. Chin, P.L. Miller, L. Zeng, K. Cawley, L.K. Weavers, Photosensitized degradation of bisphenol A by dissolved organic matter, Environmental science \& technology 38 (2004) 5888-5894.

[179] F. Vargas, M.V. Hisbeth, J.K. Rojas, Photolysis and photosensitized degradation of the diuretic drug acetazolamide, Journal of Photochemistry and Photobiology A: Chemistry 118 (1998) 19-23.

[180] H. Xu, W.J. Cooper, J. Jung, W. Song, Photosensitized degradation of amoxicillin in natural organic matter isolate solutions, Water Research 45 (2011) 632-638.

[181] L. Wang, H. Xu, W.J. Cooper, W. Song, Photochemical fate of beta-blockers in NOM enriched waters, Science of the total environment 426 (2012) 289-295.

[182] E. Díez-Mato, F. Cortezón-Tamarit, S. Bogialli, D. García-Fresnadillo, M. Marazuela, Phototransformation of model micropollutants in water samples by photocatalytic singlet oxygen production in heterogeneous medium, Applied Catalysis B: Environmental 160 (2014) 445-455. 


\section{Bibliography}

[183] R.W. Redmond, J.N. Gamlin, A compilation of singlet oxygen yields from biologically relevant molecules, Photochemistry and Photobiology 70 (1999) 391475.

[184] Q. Huang, W.-L. Fu, B. Chen, J.-F. Huang, X. Zhang, Q. Xue, Inactivation of dengue virus by methylene blue/narrow bandwidth light system, Journal of Photochemistry and Photobiology B: Biology 77 (2004) 39-43.

[185] M. Wainwright, Methylene blue derivatives-suitable photoantimicrobials for blood product disinfection?, International journal of antimicrobial agents 16 (2000) 381-394.

[186] R.A. Floyd, J.E. Schneider, D.P. Dittmer, Methylene blue photoinactivation of RNA viruses, Antiviral Research 61 (2004) 141-151.

[187] L.S. Peloi, R.R. Soares, C.E. Biondo, V.R. Souza, N. Hioka, E. Kimura, Photodynamic effect of light-emitting diode light on cell growth inhibition induced by methylene blue, Journal of biosciences 33 (2008) 231-237.

[188] A.T. Cooper, D.Y. Goswami, Evaluation of methylene blue and rose bengal for dye sensitized solar water treatment, Journal of solar energy engineering 124 (2002) 305-310.

[189] C. McCullagh, P.K. Robertson, Photosensitized destruction of Chlorella vulgaris by methylene blue or nuclear fast red combined with hydrogen peroxide under visible light irradiation, Environmental science \& technology 40 (2006) 24212425.

[190] M. Klavarioti, D. Mantzavinos, D. Kassinos, Removal of residual pharmaceuticals from aqueous systems by advanced oxidation processes, Environment International 35 (2009) 402-417.

[191] D.B. Huggett, B.W. Brooks, B. Peterson, C.M. Foran, D. Schlenk, Toxicity of Select Beta Adrenergic Receptor-Blocking Pharmaceuticals (B-Blockers) on Aquatic Organisms, Archives of Environmental Contamination and Toxicology 43 (2002) 229-235. 
[192] J.M. Poyatos, M.M. Muñio, M.C. Almecija, J.C. Torres, E. Hontoria, F. Osorio, Advanced Oxidation Processes for Wastewater Treatment: State of the Art, Water, Air, and Soil Pollution 205 (2009) 187-204.

[193] J.C. Cardoso, T.M. Lizier, M.V.B. Zanoni, Highly ordered TiO 2 nanotube arrays and photoelectrocatalytic oxidation of aromatic amine, Applied Catalysis B: Environmental 99 (2010) 96-102.

[194] Y. Zhao, N. Hoivik, K. Wang, Recent advance on engineering titanium dioxide nanotubes for photochemical and photoelectrochemical water splitting, Nano Energy 30 (2016) 728-744.

[195] X. Nie, J. Chen, G. Li, H. Shi, H. Zhao, P.K. Wong, T. An, Synthesis and characterization of $\mathrm{TiO}_{2}$ nanotube photoanode and its application in photoelectrocatalytic degradation of model environmental pharmaceuticals, Journal of Chemical Technology and Biotechnology 88 (2013) 1488-1497.

[196] H.-F. Zhuang, C.-J. Lin, Y.-K. Lai, L. Sun, J. Li, Some Critical Structure Factors of Titanium Oxide Nanotube Array in Its Photocatalytic Activity, Environmental science \& technology 41 (2007) 4735-4740.

[197] W.-K. Jo, R.J. Tayade, New Generation Energy-Efficient Light Source for Photocatalysis: LEDs for Environmental Applications, Industrial \& Engineering Chemistry Research 53 (2014) 2073-2084.

[198] T.S. Natarajan, M. Thomas, K. Natarajan, H.C. Bajaj, R.J. Tayade, Study on UVLED/TiO2 process for degradation of Rhodamine $B$ dye, Chemical Engineering Journal 169 (2011) 126-134.

[199] H.-W. Chen, Y. Ku, A. Irawan, Photodecomposition of o-cresol by UV-LED/TiO2 process with controlled periodic illumination, Chemosphere 69 (2007) 184-190.

[200] K. Natarajan, T.S. Natarajan, H.C. Bajaj, R.J. Tayade, Photocatalytic reactor based on UV-LED/TiO2 coated quartz tube for degradation of dyes, Chemical Engineering Journal 178 (2011) 40-49. 


\section{Bibliography}

[201] K. Yasuda, P. Schmuki, Control of morphology and composition of selforganized zirconium titanate nanotubes formed in (NH 4) 2 SO 4/NH 4 F electrolytes, Electrochimica Acta 52 (2007) 4053-4061.

[202] J. Macak, H. Hildebrand, U. Marten-Jahns, P. Schmuki, Mechanistic aspects and growth of large diameter self-organized TiO 2 nanotubes, Journal of Electroanalytical Chemistry 621 (2008) 254-266.

[203] H.-c. Liang, X.-z. Li, Effects of structure of anodic TiO2 nanotube arrays on photocatalytic activity for the degradation of 2,3-dichlorophenol in aqueous solution, Journal of Hazardous Materials 162 (2009) 1415-1422.

[204] S. Sreekantan, R. Hazan, Z. Lockman, Photoactivity of anatase-rutile TiO2 nanotubes formed by anodization method, Thin Solid Films 518 (2009) 16-21.

[205] D.W. Newton, R.B. Kluza, pKa values of medicinal compounds in pharmacy practice, Annals of Pharmacotherapy 12 (1978) 546-554.

[206] W.W.-P. Lai, M.-H. Hsu, A.Y.-C. Lin, The role of bicarbonate anions in methotrexate degradation via UV/TiO2: Mechanisms, reactivity and increased toxicity, Water Research 112 (2017) 157-166.

[207] G.V. Buxton, C.L. Greenstock, W.P. Helman, A.B. Ross, Critical Review of rate constants for reactions of hydrated electrons, hydrogen atoms and hydroxyl radicals ( $\cdot \mathrm{OH} / \cdot \mathrm{O}-$ in Aqueous Solution, Journal of Physical and Chemical Reference Data 17 (1988) 513-886.

[208] Y. Ku, W.-H. Lee, W.-Y. Wang, Photocatalytic reduction of carbonate in aqueous solution by UV/TiO2 process, Journal of Molecular Catalysis A: Chemical 212 (2004) 191-196.

[209] D. Zhao, C. Chen, Y. Wang, H. Ji, W. Ma, L. Zang, J. Zhao, Surface modification of TiO2 by phosphate: effect on photocatalytic activity and mechanism implication, The Journal of Physical Chemistry C 112 (2008) 5993-6001.

[210] Y. Ye, Y. Feng, H. Bruning, D. Yntema, H.H.M. Rijnaarts, Photocatalytic degradation of metoprolol by TiO2 nanotube arrays and UV-LED: Effects of catalyst 
properties, operational parameters, commonly present water constituents, and photo-induced reactive species, Applied Catalysis B: Environmental 220 (2018) 171181.

[211] S.L. Gora, S.A. Andrews, Adsorption of natural organic matter and disinfection byproduct precursors from surface water onto $\mathrm{TiO} 2$ nanoparticles: $\mathrm{pH}$ effects, isotherm modelling and implications for using $\mathrm{TiO} 2$ for drinking water treatment, Chemosphere 174 (2017) 363-370.

[212] J. Brame, M. Long, Q. Li, P. Alvarez, Inhibitory effect of natural organic matter or other background constituents on photocatalytic advanced oxidation processes: Mechanistic model development and validation, Water Research 84 (2015) 362-371. [213] M. Erhayem, M. Sohn, Stability studies for titanium dioxide nanoparticles upon adsorption of Suwannee River humic and fulvic acids and natural organic matter, Science of the total environment 468-469 (2014) 249-257.

[214] C.S. Uyguner-Demirel, N.C. Birben, M. Bekbolet, Elucidation of background organic matter matrix effect on photocatalytic treatment of contaminants using TiO2: A review, Catal Today 284 (2017) 202-214.

[215] C. Guillard, E. Puzenat, H. Lachheb, A. Houas, J.-M. Herrmann, Why inorganic salts decrease the $\mathrm{TiO} 2$ photocatalytic efficiency, International Journal of Photoenergy 7 (2005) 1-9.

[216] I. Harrison, R.U. Leader, J.J.W. Higgo, G.M. Williams, A study of the degradation of phenoxyacid herbicides at different sites in a limestone aquifer, Chemosphere 36 (1998) 1211-1232.

[217] D. Vione, S. Khanra, R. Das, C. Minero, V. Maurino, M. Brigante, G. Mailhot, Effect of dissolved organic compounds on the photodegradation of the herbicide MCPA in aqueous solution, Water Research 44 (2010) 6053-6062.

[218] F. Orton, I. Lutz, W. Kloas, E.J. Routledge, Endocrine Disrupting Effects of Herbicides and Pentachlorophenol: In Vitro and in Vivo Evidence, Environmental science \& technology 43 (2009) 2144-2150. 


\section{Bibliography}

[219] S. Tufi, P.N.H. Wassenaar, V. Osorio, J. de Boer, P.E.G. Leonards, M.H. Lamoree, Pesticide Mixture Toxicity in Surface Water Extracts in Snails (Lymnaea stagnalis) by an in Vitro Acetylcholinesterase Inhibition Assay and Metabolomics, Environmental science \& technology 50 (2016) 3937-3944.

[220] A. Butkovskyi, A.W. Jeremiasse, L. Hernandez Leal, T. van der Zande, H. Rijnaarts, G. Zeeman, Electrochemical Conversion of Micropollutants in Gray Water, Environmental science \& technology 48 (2014) 1893-1901.

[221] P. Mwaanga, E.R. Carraway, M.A. Schlautman, Preferential sorption of some natural organic matter fractions to titanium dioxide nanoparticles: influence of $\mathrm{pH}$ and ionic strength, Environmental Monitoring and Assessment 186 (2014) 88338844.

[222] D. Jiang, H. Zhao, S. Zhang, R. John, Kinetic study of photocatalytic oxidation of adsorbed carboxylic acids at $\mathrm{TiO} 2$ porous films by photoelectrolysis, Journal of Catalysis 223 (2004) 212-220.

[223] Y. Xiaoju, B. Ruiling, Y. Shuili, Effect of inorganic ions on the photocatalytic degradation of humic acid, Russian Journal of Physical Chemistry A 86 (2012) 13181325.

[224] E.K. Radwan, L. Yu, G. Achari, C.H. Langford, Photocatalytic ozonation of pesticides in a fixed bed flow through UVA-LED photoreactor, Environmental Science and Pollution Research 23 (2016) 21313-21318.

[225] S. Irmak, E. Kusvuran, O. Erbatur, Degradation of 4-chloro-2-methylphenol in aqueous solution by UV irradiation in the presence of titanium dioxide, Applied Catalysis B: Environmental 54 (2004) 85-91.

[226] C. Pablos, J. Marugán, R. van Grieken, P. Dunlop, J. Hamilton, D. Dionysiou, J. Byrne, Electrochemical Enhancement of Photocatalytic Disinfection on Aligned TiO2 and Nitrogen Doped TiO2 Nanotubes, Molecules 22 (2017) 704. 
[227] R.A.R. Monteiro, F.V.S. Lopes, R.A.R. Boaventura, A.M.T. Silva, V.J.P. Vilar, Synthesis and characterization of $\mathrm{N}$-modified titania nanotubes for photocatalytic applications, Environmental Science and Pollution Research 22 (2015) 810-819.

[228] P. Lianos, Production of electricity and hydrogen by photocatalytic degradation of organic wastes in a photoelectrochemical cell: The concept of the Photofuelcell: A review of a re-emerging research field, Journal of Hazardous Materials 185 (2011) 575-590.

[229] P. Lianos, Review of recent trends in photoelectrocatalytic conversion of solar energy to electricity and hydrogen, Applied Catalysis B: Environmental 210 (2017) 235-254.

[230] I. Tantis, M. Antonopoulou, I. Konstantinou, P. Lianos, Coupling of electrochemical and photocatalytic technologies for accelerating degradation of organic pollutants, Journal of Photochemistry and Photobiology A: Chemistry 317 (2016) 100-107.

[231] M. Antoniadou, D.I. Kondarides, D.D. Dionysiou, P. Lianos, Quantum Dot Sensitized Titania Applicable as Photoanode in Photoactivated Fuel Cells, The Journal of Physical Chemistry C 116 (2012) 16901-16909.

[232] K. Zhao, Q. Zeng, J. Bai, J. Li, L. Xia, S. Chen, B. Zhou, Enhanced organic pollutants degradation and electricity production simultaneously via strengthening the radicals reaction in a novel Fenton-photocatalytic fuel cell system, Water Research 108 (2017) 293-300.

[233] Y. Zhao, N. Hoivik, K. Wang, Recent advance on engineering titanium dioxide nanotubes for photochemical and photoelectrochemical water splitting, Nano Energy (2016).

[234] Q. Chen, H. Liu, Y. Xin, X. Cheng, Coupling immobilized TiO2 nanobelts and Au nanoparticles for enhanced photocatalytic and photoelectrocatalytic activity and mechanism insights, Chemical Engineering Journal 241 (2014) 145-154. 


\section{Bibliography}

[235] S.L. Gora, S.A. Andrews, Adsorption of natural organic matter and disinfection byproduct precursors from surface water onto $\mathrm{TiO} 2$ nanoparticles: $\mathrm{pH}$ effects, isotherm modelling and implications for using $\mathrm{TiO} 2$ for drinking water treatment, Chemosphere 174 (2017) 363-370.

[236] K. Zhao, J. Bai, Q. Zeng, Y. Zhang, J. Li, L. Li, L. Xia, B. Zhou, Efficient wastewater treatment and simultaneously electricity production using a photocatalytic fuel cell based on the radical chain reactions initiated by dual photoelectrodes, Journal of Hazardous Materials 337 (2017) 47-54.

[237] S. Liu, X. Zhao, H. Zeng, Y. Wang, M. Qiao, W. Guan, Enhancement of photoelectrocatalytic degradation of diclofenac with persulfate activated by $\mathrm{Cu}$ cathode, Chemical Engineering Journal 320 (2017) 168-177.

[238] Y. Liu, J. Li, B. Zhou, S. Lv, X. Li, H. Chen, Q. Chen, W. Cai, Photoelectrocatalytic degradation of refractory organic compounds enhanced by a photocatalytic fuel cell, Applied Catalysis B: Environmental 111-112 (2012) 485-491.

[239] A. Piscopo, D. Robert, J.V. Weber, Influence of $\mathrm{pH}$ and chloride anion on the photocatalytic degradation of organic compounds: Part I. Effect on the benzamide and para-hydroxybenzoic acid in $\mathrm{TiO}_{2}$ aqueous solution, Applied Catalysis B: Environmental 35 (2001) 117-124.

[240] Z.H. Mussa, F.F. Al-Qaim, M.R. Othman, M.P. Abdullah, J. Latip, Z. Zakria, Pseudo first order kinetics and proposed transformation products pathway for the degradation of diclofenac using graphite-PVC composite as anode, Journal of the Taiwan Institute of Chemical Engineers 72 (2017) 37-44.

[241] S. Encinas, F. Bosca, M.A. Miranda, Phototoxicity Associated with Diclofenac: A Photophysical, Photochemical, and Photobiological Study on the Drug and Its Photoproducts, Chemical Research in Toxicology 11 (1998) 946-952.

[242] J. Kuipers, H. Bruning, D. Yntema, H. Rijnaarts, Wirelessly powered ultraviolet light emitting diodes for photocatalytic oxidation, Journal of Photochemistry and Photobiology A: Chemistry 299 (2015) 25-30. 
[243] S. Verma, M. Sillanpää, Degradation of anatoxin-a by UV-C LED and UV-C LED/H2O2 advanced oxidation processes, Chemical Engineering Journal 274 (2015) 274-281.

[244] C.S. Foote, Photosensitized oxidation and singlet oxygen: consequences in biological systems, Free radicals in biology 2 (1976) 85-133.

[245] R. Gerdes, D. Wöhrle, W. Spiller, G. Schneider, G. Schnurpfeil, G. Schulz-Ekloff, Photo-oxidation of phenol and monochlorophenols in oxygen-saturated aqueous solutions by different photosensitizers, Journal of Photochemistry and Photobiology A: Chemistry 111 (1997) 65-74.

[246] M.A. Miranda, F. Galindo, A.M. Amat, A. Arques, Pyrylium saltphotosensitized degradation of phenolic contaminants derived from cinnamic acid with solar light: Correlation of the observed reactivities with fluorescence quenching, Applied Catalysis B: Environmental 28 (2000) 127-133.

[247] M. Sánchez-Polo, M.M. Abdel daiem, R. Ocampo-Pérez, J. Rivera-Utrilla, A.J. Mota, Comparative study of the photodegradation of bisphenol A by HO, SO4- and CO3-/HCO3 radicals in aqueous phase, Science of the total environment 463-464 (2013) 423-431.

[248] S. Canonica, T. Kohn, M. Mac, F.J. Real, J. Wirz, U. von Gunten, Photosensitizer Method to Determine Rate Constants for the Reaction of Carbonate Radical with Organic Compounds, Environmental science \& technology 39 (2005) 9182-9188.

[249] A. Knowles, A mechanism for the methylene blue sensitized oxidation of nucleotides, Photochemistry and Photobiology 13 (1971) 473-487.

[250] J. Chen, T.C. Cesario, P.M. Rentzepis, Effect of pH on methylene blue transient states and kinetics and bacteria photoinactivation, The Journal of Physical Chemistry A 115 (2011) 2702-2707.

[251] A. Acher, S. Saltzman, Dye-sensitized photooxidation of bromacil in water, Journal of Environmental Quality 9 (1980) 190-194. 


\section{Bibliography}

[252] H. Yu, E. Nie, J. Xu, S. Yan, W.J. Cooper, W. Song, Degradation of diclofenac by advanced oxidation and reduction processes: kinetic studies, degradation pathways and toxicity assessments, Water Research 47 (2013) 1909-1918.

[253] A. Agüera, L. Perez Estrada, I. Ferrer, E. Thurman, S. Malato, A. Fernández Alba, Application of time - of - flight mass spectrometry to the analysis of phototransformation products of diclofenac in water under natural sunlight, Journal of Mass Spectrometry 40 (2005) 908-915.

[254] P. Bartels, W. von Tümpling, Solar radiation influence on the decomposition process of diclofenac in surface waters, Science of the total environment 374 (2007) 143-155.

[255] C.D. Herzfeldt, R. Kümmel, Dissociation constants, solubilities and dissolution rates of some selected nonsteroidal antiinflammatories, Drug Development and Industrial Pharmacy 9 (1983) 767-793.

[256] P.M. Dewick, Essentials of Organic Chemistry: For Students of Pharmacy, Medicinal Chemistry and Biological Chemistry, Wiley2013.

[257] X. Zhu, J. Zhou, Z. Cai, TiO2 Nanoparticles in the Marine Environment: Impact on the Toxicity of Tributyltin to Abalone (Haliotis diversicolor supertexta) Embryos, Environmental science \& technology 45 (2011) 3753-3758.

[258] L. Clément, C. Hurel, N. Marmier, Toxicity of TiO2 nanoparticles to cladocerans, algae, rotifers and plants - Effects of size and crystalline structure, Chemosphere 90 (2013) 1083-1090.

[259] M. Pelaez, N.T. Nolan, S.C. Pillai, M.K. Seery, P. Falaras, A.G. Kontos, P.S.M. Dunlop, J.W.J. Hamilton, J.A. Byrne, K. O'Shea, M.H. Entezari, D.D. Dionysiou, A review on the visible light active titanium dioxide photocatalysts for environmental applications, Applied Catalysis B: Environmental 125 (2012) 331-349.

[260] A.I. Kontos, A.G. Kontos, Y.S. Raptis, P. Falaras, Nitrogen modified nanostructured titania: electronic, structural and visible - light photocatalytic properties, physica status solidi (RRL) - Rapid Research Letters 2 (2008) 83-85. 
[261] P. Periyat, S.C. Pillai, D.E. McCormack, J. Colreavy, S.J. Hinder, Improved HighTemperature Stability and Sun-Light-Driven Photocatalytic Activity of Sulfur-Doped Anatase TiO2, The Journal of Physical Chemistry C 112 (2008) 7644-7652.

[262] T. Umebayashi, T. Yamaki, S. Tanaka, K. Asai, Visible light-induced degradation of methylene blue on S-doped TiO2, Chemistry Letters 32 (2003) 330-331.

[263] T. Boningari, S.N.R. Inturi, M. Suidan, P.G. Smirniotis, Novel one-step synthesis of sulfur doped-TiO2 by flame spray pyrolysis for visible light photocatalytic degradation of acetaldehyde, Chemical Engineering Journal 339 (2018) 249-258.

[264] S. Feizpoor, A. Habibi-Yangjeh, S. Vadivel, Novel TiO2/Ag2CrO4 nanocomposites: Efficient visible-light-driven photocatalysts with $n-n$ heterojunctions, Journal of Photochemistry and Photobiology A: Chemistry 341 (2017) 57-68.

[265] X. Chang, T. Wang, J. Gong, CO2 photo-reduction: insights into CO2 activation and reaction on surfaces of photocatalysts, Energy \& Environmental Science 9 (2016) 2177-2196.

[266] K. Li, X. An, K.H. Park, M. Khraisheh, J. Tang, A critical review of $\mathrm{CO} 2$ photoconversion: Catalysts and reactors, Catal Today 224 (2014) 3-12. 


\section{List of abbreviations}


AOP: Advanced oxidation processes

DDT: Dichorodiphenyltrichloroethane

DFN: Diclofenac

DO: Dissolved oxygen

HCB: Hexachlorobenzene

LED: Light-emitting diode

MB: Methylene blue

MCPA: 4-chloro-2-methylphenoxyacetic acid

MTP: Metoprolol

NOM: Natural organic matter

PC: Photocatalysis

PCB: Polychlorinated biphenyl

PEC: Photoelectrocatalysis

PFC: Photocatalytic fuel cell

PRP: Propranolol

ROS: Reactive oxygen species

SFZ: Sulfamethoxazole

TNA: $\quad \mathrm{TiO}_{2}$ nanotube array

TNT: $\quad \mathrm{TiO}_{2}$ nanotube

TP: Transformation product

UV: Ultraviolet

WWTP: Wastewater treatment plant 


\section{Summary}




\section{Summary}

As described in Chapter 1, the increasing legislation pressure, risks associated with micropollutants, and the insufficient efficacy of conventional WWTPs on their removal have formed a joint driving force that is boosting studies on more efficient treatment technology. The general objective of this thesis is to contribute to a better understanding and further development of photochemical treatment processes for micropollutant removal from aqueous streams. Emphasis of the experimental work of this thesis is given to two topics: (1) Further development of UV/ $\mathrm{TiO}_{2}$ technique by application of TNA as immobilized catalyst; (2) Development of alternative photochemical water treatment technique for micropollutant removal from wastewater.

In Chapter 2, the use of self-organized $\mathrm{TiO}_{2}$ nanotube arrays (TNAs) as immobilized catalyst for photocatalytic degradation of micropollutant was evaluated. The $\beta$-blocker metoprolol (MTP) was chosen as a model micropollutant. Firstly the synthesis of selforganized TNA was studied, where the effect of anodization potential and annealing temperature was examined. Characterization by SEM demonstrated a linear relation between the diameter of $\mathrm{TiO}_{2}$ nanotubes produced and the anodization potential, while Raman measurement revealed the vital role of annealing on crystallographic composition of the anodic produced $\mathrm{TiO}_{2}$ nanotubes. Regarding their performance in photocatalytic MTP degradation, surface morphology and crystallographic composition of the TNAs were found to impose crucial influence: only TNAs with diameter not smaller than $53 \mathrm{~nm}$ enabled rapid MTP degradation, and highest MTP degradation was obtained when a mixture of anatase and rutile were present in the TNAs. Secondly, the effect of operational parameters, i.e initial MTP concentration, $\mathrm{pH}$, was investigated. Rapid MTP degradation and high total removal were achieved in a wide $\mathrm{pH}$ range (3-11) and even with low initial MTP concentration. To evaluate the applicability of TNAs for water treatment, experiments were first carried out in the presence of three different commonly present water constituents, i.e bicarbonate ions, phosphate ions, and natural organic matters (NOMs). The results show that bicarbonate and phosphate ions have no inhibitory effect at concentration levels up to $200 \mathrm{mg} / \mathrm{L}$, and NOMs exhibit detrimental effect when their concentration exceeds $5 \mathrm{mg} / \mathrm{L}$. The total removal MTP degradation reduced from $87.09 \%$ to $62.05 \%$ when tap water 
samples were applied, demonstrating reasonable efficacy for practical applications. Regarding the degradation mechanism, formic acid and tert-butanol were added as scavenger for photo-generated holes $\left(\mathrm{h}^{+}\right)$and hydroxyl radicals $(\cdot \mathrm{OH})$, respectively. The obtained results demonstrate that primary degradation process occurred in liquid phase with participation of hydroxyl radicals in the liquid phase $(\cdot \mathrm{OH}$ liquid), while smaller portion of MTP were degraded on the catalysis surface via reaction with $\mathrm{h}^{+}$and hydroxyl radicals adsorbed on the catalyst surface $(\cdot \mathrm{OH}$ surface). Other reactive species, e.g. photo-generated electrons and superoxide radical anions, did also play a minor role in MTP degradation. The mechanistic aspect was further confirmed by identification of degradation products by LCMS/MS. The TNA exhibited good stability after repeated use under varied operation conditions.

Chapter 3 aimed at better understanding on the applicability of TNA in complex water matrices. This chapter investigated the effect of dissolved natural organic matter (NOMs) on MCPA (a typical micropollutant found in many water bodies) removal performance of TNA. The present study shows that although in bulk liquid phase NOMs would undergo photosensitization that can contribute to MCPA removal, the overall effect of NOMs on MCPA removal is detrimental due to the interaction between NOMs and the TNA surface. An acidic $\mathrm{pH}$ however, was found to be able to mitigate the detrimental effect of NOMs, and the photosensitization effect of NOMs was strengthened; while under alkaline $\mathrm{pH}$ conditions completely the detrimental effect of NOMs vanished. Two commonly present co-existing anions, i.e. phosphate and bicarbonate, could also mitigate the detrimental effect of NOMs, and the photocurrent measurement support that the presence of such anions could greatly suppress the $h+$ scavenging effect of NOMs; while other anions, i.e. chloride, nitrate, sulfate, showed no notable effect.

Another major drawback of $\mathrm{TiO}_{2}$ photocatalysis is the fast recombination of $\mathrm{e}^{-/} / \mathrm{h}^{+}$ pairs, where a large amount of energy from absorbed photons is lost as heat, resulting in seriously reduced treatment efficiency. Chapter 4 demonstrated enhanced micropollutant (MCPA as model pollutant) degradation in a membrane-free PFC system consisting of a TNA photoanode and a $\mathrm{Cu}$ foil acting as cathode (TNA-CU PFC). The TNA-Cu PFC system showed better MCPA degradation compared to the conventional photocatalytic method using the 


\section{Summary}

same catalyst. In the TNA-Cu PFC system, the MCPA degradation was largely promoted under acidic conditions, indicating this as an important operational condition. The enhancement of MCPA degradation in the TNA-CU PFC system involved better $\mathrm{e}^{-} / \mathrm{h}^{+}$ separation and generation of other oxidants: in conventional photocatalytic process, hydroxyl radicals in liquid phase contributed to $93.7 \%$ MCPA degradation while only $2.4 \%$ MCPA degradation was mediated by other oxidants like $\cdot \mathrm{O}_{2}, \mathrm{H}_{2} \mathrm{O}_{2}, \cdot \mathrm{HO}_{2}$; for $\mathrm{MCPA}$ degradation in the TNA-Cu PFC system, the contribution of hydroxyl radicals in the liquid phase decreased to $83.6 \%$, while contribution of other oxidants like $\cdot \mathrm{O}_{2}{ }^{-}, \mathrm{H}_{2} \mathrm{O}_{2}, \cdot \mathrm{HO}_{2}$ increased to $15.3 \%$. This change in MCPA degradation mechanisms was further confirmed via analysis of degradation intermediates by LC-MS/MS. A study on the effect of electrolyte concentration suggests that when operated under acidic conditions, addition of electrolyte is not required. The TNA-CU PFC system was shown to work well in the presence of up to 15 $\mathrm{mg} / \mathrm{L}$ natural organic matter (originating from two large rivers), high amounts of common inorganic ions, and even in WWTP effluent. The TNA-Cu PFC system also exhibited relatively good stability after several cycles of repeated use. The obtained results demonstrated that this is an adequate system for micropollutant removal from water at various places in the water cycle, i.e. as polisher of WWTP effluents before discharge or for cleaning intake water before producing drinking water.

Chapter 5 reported red light induced methylene blue photosensitization (MB/Redlight) as a promising alternative for pharmaceuticals removal from wastewater, because of its potential to overcome the detrimental effect of background water constituents as experienced in other AOP technologies. In this study, the efficacy of MB/Red-light on degradation of four pharmaceutical compounds, i.e. diclofenac (DFN), metoprolol (MTP), propranolol (PRP) and sulfamethoxazole (SFZ), was investigated. The MB/Red-light photosensitization enabled degradation of three model compounds, i.e. DFN, PRP and SFZ. Degradation rates followed the order of DFN $>$ PRP $>$ SFZ. Singlet oxygen was found to be crucial in pharmaceuticals degradation, and another additional mechanism, i.e. a direct reaction with triplet $\mathrm{MB}$, also contributed to DFN degradation. The presence of two DFN degradation mechanisms were confirmed by UV-vis light absorbance spectra measurement as well as the identification of degradation products by LC-MS/MS. Effects of three common back ground water constituents were examined to assess the applicability of $\mathrm{MB} / \mathrm{red}$-light 
system in complex water matrices, which suggests that the $M B /$ red-light system has great potential to be used in real wastewater. Higher $\mathrm{pH}$ was found to impose positive impact on the efficacy of the proposed system. Last but not least, red light LED is an optimum light source for the proposed MB/Red-light system, because the light emission spectrum of the LED used in this study fits well with the light absorption spectrum of the photosensitizermethylene blue.

To further develop the MB/Red-light technique, Chapter 6 aimed at studying the effect of operational parameters on the performance of $\mathrm{MB} /$ Red-light micropollutant removal processes. Three pharmaceuticals, i.e. diclofenac (DFN), propranolol (PRP), and sulfamethoxazole (SFZ), were used as model compounds, and degradation rate constants and total compound removal were examined. The three operational parameters studied were initial $\mathrm{MB}$ concentration $(0-5.0 \mathrm{mg} / \mathrm{L})$, initial pharmaceutical concentration $(0.1-2.0$ $\mathrm{mg} / \mathrm{L})$, aeration rate $(0-5.0 \mathrm{~L} / \mathrm{min})$, and for DFN also the $\mathrm{pH}$. The results of pharmaceuticals tested show that values of both degradation rate constants and the total removal increase with increasing initial MB concentration at values of $[\mathrm{MB}]$ below $0.5 \mathrm{mg} / \mathrm{l}$, and leveled off to constant values at $[\mathrm{MB}]$ values higher than $2.0 \mathrm{mg} / \mathrm{l}$. Initial pharmaceutical concentration and aeration rate were found to have no significant impact. Moreover, rapid degradation of pharmaceuticals can take place even at low initial dissolved oxygen concentrations (2.0 $\mathrm{mg} / \mathrm{L}$, i.e. situations without aeration). In order to better understand the effect of $\mathrm{pH}$ on the $\mathrm{MB} /$ Red-light pharmaceutical degradation processes, DFN was chosen for more detailed investigation, with identification of the degradation products formed under neutral and alkaline conditions identified by LC-MS/MS. The $\mathrm{pH}$ was found to play an important role on the transformation pathways and formation of degradation products.

The main outcomes of the thesis are discussed in Chapter $\mathbf{7}$ and further research opportunities were identified. Considering full-scale application of the studied techniques, further research efforts are suggested on: (1) stability of TNA after long time exposure to complex water matrices and complex hydraulic conditions; (2) by-products formation and toxicity test; (3) exploring the limits of $\mathrm{MB} /$ Red-light technique; (4) process optimization and reactor design. New research opportunities for improving the sustainability are also identified: (1) use of solar light; (2) integrating water treatment with resource recovery. 


\section{Acknowledgements}


Finally, after almost four years, my PhD project comes to an end. I would not be able to finish this bittersweet journey without the support of my promotor and supervisors, my colleagues, my friends, and, most importantly, my family.

First of all, I would like to express my sincere gratitude to my beloved family. 感谢 我的父母, 你们为了我的成长和发展, 在过去的几十年中, 付出了极大的心血, 做 出了极大的牺牲。你们给予我无私的爱, 竭尽所能给予我精神和经济上的支持和帮 助, 让我的求学之路和人生之路更加顺畅。我会时刻谨记教诲, 修身明德, 择善而 从，慎思明辨，做一个于社会和国家有用的人。感谢我的妻子，你对我的爱、陪伴 和支持, 是我生命中所得到过的最美好的馈赠。我在外留学的这几年, 我们为了各 自的事业不得不聚少离多, 但你毫无怨言, 一直在默默地支持我, 默默地为我们的 小家付出着; 没有你的理解和支持, 就不会有这一本博士论文。

My sincere appreciation goes to my supervision team-Huub, Harry, and Doekle. Huub, I would like to thank you for your support and guidance during all these years. Your expertise in water treatment and scientific research contributed significantly on my project. It is a great pleasure and my honor to be your student. Harry, when I first contacted you to apply for this PhD position, I knew very little about photocatalysis, but you guided me into this field, gave me freedom to explore this wonderful field, and allowed me to grow into a qualified researcher. You have very good sense of humor; I enjoyed all the nice jokes during our discussions. Thank you for all the guidance and help. Doekle, you are always very cheerful and optimistic. The journey of PhD was sometimes quite challenging, but you were always there encouraging me when I face problems. Without your help, my PhD journey would be more difficult. I also learned a lot from you on how to enjoy life. Thank you for all the support and supervision at Wetsus; it is a great experience to work with you.

Thank you my office mates, Antoine, Casper, Enas, Ilse, Jorrit, Louis, Naghme, Natalia, Suyash, Dr. Semitsoglou-Tsiapou, Tim, Tania, Vinu, Zexin, you guys brought in lots of happiness to the office. The good atmosphere of the "office of happiness" made my daily work much easier. To my other Wetsus colleagues and friends, Hakan, Hector, Prashanth, 
Yang, Gaofeng, Qingdian, and all other friends, thank you for the nice time we spent together.

I would like to thank the participants of the research theme "Advanced water treatment" of Wetsus for the fruitful discussions and their financial support. I would also like to thank the lab support team of Wetsus, Ton van der Zande, Mieke Kersaan-Han and anyone else for realizing the instrumental analysis, and Ernst Panstra, John Ferwerda, and Jan Tuinstra and anyone else for the technical support in the experimental hall. I could not forget MSc students: Yujia, Yanyue, Xiaolu, and Wanrong. I am grateful for their hardworking and contribution on my project.

I would like to thank my Wageningen friends, Lei, Bingnan, Cong Bao, Si, Yongran, for all the nice companies every time I went there. Special thanks to my "Shifu" and "Shimu": Momo and Dandan, for your hospitality and all the nice meals. Thank the micropollutant group of ETE, Yujie, Wenbo, Arnoud, Alette, Nora, for their support and nice discussions. I would like to thank all ETE study trip (2016) participants for the happy moments we had in China. 


\section{About the author}


Yin Ye was born on December 28 $8^{\text {th }}, 1989$ in Sichuan, China. In 2008 he graduated from Deyang Middle School, after which he entered Southwest Jiaotong University to study Environmental Engineering. Four years later, he was awarded the Bachelor of Engineering. The bachelor thesis was about reuse of lignin from paper mill wastewater. After receiving the bachelor's degree, he entered Wageningen University as a student of MSc programme in Environmental Sciences (specialized in Environmental Technology). He finished his MSc with a thesis entitled "Feasibility study of methanol-based chain elongation in mixed culture" at the sub-department of Environmental Technology and an internship at the Hong Kong Polytechnic University on cement-free soil improvement techniques in 2014. In 2015, he continued as a PhD student at the sub-department of Environmental Technology of Wageningen University and Wetsus, under supervision of Dr Harry Bruning, Dr Doekle Yntema, and Prof. Dr Huub Rijnaarts. His PhD project aimed at removal of micropollutants from aqueous streams by using photochemical processes. 
List of publications

\section{List of publications}


List of publications

\section{Scientific journal articles}

\section{In relation to this thesis}

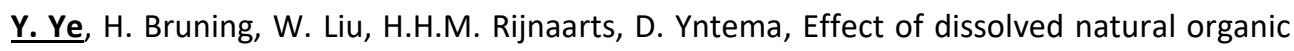
matter on the photocatalytic micropollutant removal performance of $\mathrm{TiO}_{2}$ nanotube array, Submitted.

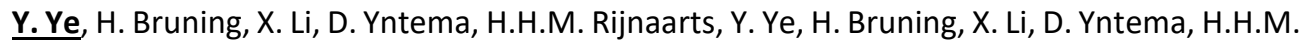
Rijnaarts, Significant enhancement of micropollutant photocatalytic degradation using a TiO2 nanotube array photoanode based photocatalytic fuel cell, Chemical Engineering Journal 354 (2018) 553-562.

Y. Ye, Y. Feng, H. Bruning, D. Yntema, H.H.M. Rijnaarts, Photocatalytic degradation of metoprolol by $\mathrm{TiO} 2$ nanotube arrays and UV-LED: effects of catalyst properties, operational parameters, commonly present water constituents, and photo-induced reactive species, Applied Catalysis B: Environmental 220C (2018) 171-181.

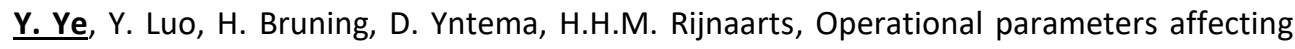
$\mathrm{MB} /$ Red-light photosensitized degradation of pharmaceuticals, Journal of Photochemistry \& Photobiology, A: Chemistry 348C (2017) 96-101.

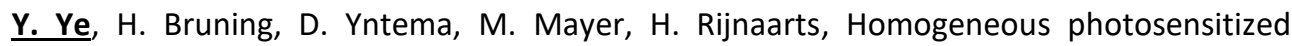
degradation of pharmaceuticals by using red light LED as light source and methylene blue as photosensitizer, Chemical Engineering Journal 316 (2017) 872-881.

\section{Other articles}

W. Chen, $\underline{\mathbf{Y} . \text { Ye }}$, K. Steinbusch, D. Strik, C. Buisman, Methanol as an alternative electron donor in chain elongation for butyrate and caproate formation, Biomass and Bioenergy 93 (2016) 201-208. 


\section{Conference contributions}

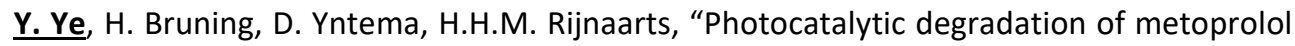
by $\mathrm{TiO}_{2}$ nanotube arrays (TNA)", Wetsus Congress, November 30, 2017, Leeuwarden, The Netherlands (Oral)

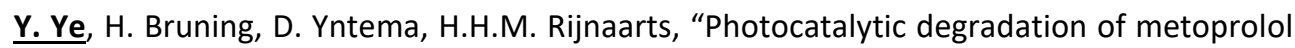
by $\mathrm{TiO}_{2}$ nanotube arrays (TNA)", Wetsus Congress, October 9-10, 2017, Leeuwarden, The Netherlands (Oral)

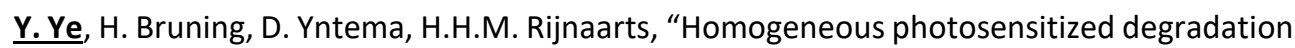
of pharmaceuticals by using red light LED as light source and methylene blue as photosensitizer", The 1st International Conference on Sustainable Water Processing, September 11 - 14, 2016, Sitges, Spain (Oral)

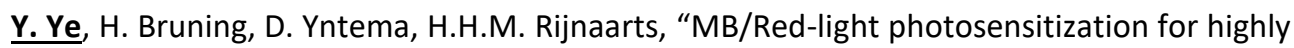
selective micropollutants removal", The CHINED-4D Environmental Solutions Symposium, June 5-18, 2016, Xi`an, China (Oral)

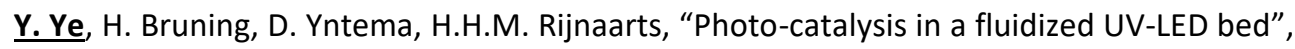
Environmental Technology for Impact 2015, April 29 - 30, 2015, Wageningen, The Netherlands (Poster) 


\section{SENSE}

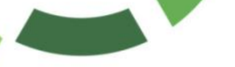

Netherlands Research School for the

Socio-Economic and Natural Sciences of the Environment

\section{I P L O M A}

For specialised PhD training

The Netherlands Research School for the Socio-Economic and Natural Sciences of the Environment (SENSE) declares that

\section{Yin Ye}

born on 28 December 1989 year in Sichuan, China

has successfully fulfilled all requirements of the Educational Programme of SENSE.

Wageningen, 7 December 2018

On behalf of the SENSE board

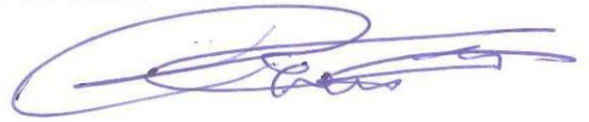

Prof. dr. Huub Rijnaarts the SENSE Director of Education

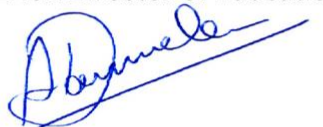

Dr. Ad van Dommelen

The SENSE Research School has been accredited by the Royal Netherlands Academy of Arts and Sciences (KNAW)

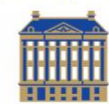

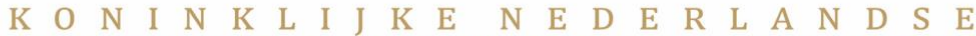
$\begin{array}{llllllllllllllllllllllll}\text { A } & K & A & D & E & M & I & E & V & A & N & \text { W } & E & T & E & N & S & C & H & A & P & P & E & N\end{array}$ 


\section{SENSE}

The SENSE Research School declares that Yin Ye has successfully fulfilled all requirements of the Educational PhD Programme of SENSE with a work load of $37.7 \mathrm{EC}$, including the following activities:

\section{SENSE PhD Courses}

- Environmental research in context (2015)

- Research in context activity: 'Co-organizing the CHI-NED 4D Dialogue Seminar and a PhD study trip to China, June 4th-18th, 2016'

\section{Other PhD and Advanced MSc Courses}

- Presentation skills, Wetsus (2015)

- Supervision of thesis students by PhD Students, Wetsus (2015)

- Illustrations for scientific publications, Wetsus (2017)

- Design of experiments, Wetsus (2017)

\section{Other courses}

- Water and wastewater treatment engineering: biochemical technology, Tsinghua University EdX course (2018)

\section{Management and Didactic Skills Training}

o Supervising four MSc students with thesis (2015-2018)

\section{Oral Presentations}

- MB/Red-light photosensitization for highly selective micropollutants removal. The CHINED-4D Environmental Solutions Symposium, 5-18 June 2016, Beijing, Xi'an, and Shanghai, China

- Homogeneous photosensitized degradation of pharmaceuticals by using red light LED as light source and methylene blue as photosensitizer. 1st International Conference on Sustainable Water Processing, 11-14 September 2016, Sitges, Spain

- Photocatalytic degradation of metoprolol by TiO2 nanotube arrays (TNAs). Wetsus Congres; synergy in research and innovation, 9-10 October 2017, Leeuwarden, the Netherlands

SENSE Coordinator PhD Education

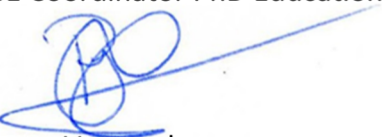

Dr. Peter Vermeulen 
This work was performed in the cooperation framework of Wetsus, European Centre of Excellence for Sustainable Water Technology (www.wetsus.eu). Wetsus is cofunded by the Dutch Ministry of Economic Affairs and Ministry of Infrastructure and Environment, the Province of Fryslân, and the Northern Netherlands Provinces. The author would like to thank the participants of the research theme "Advanced water treatment" for the fruitful discussions and their financial support.

Cover painting is by Yujia Luo and Xin Liao.

Printed by: Digiforce || ProefschriftMake 



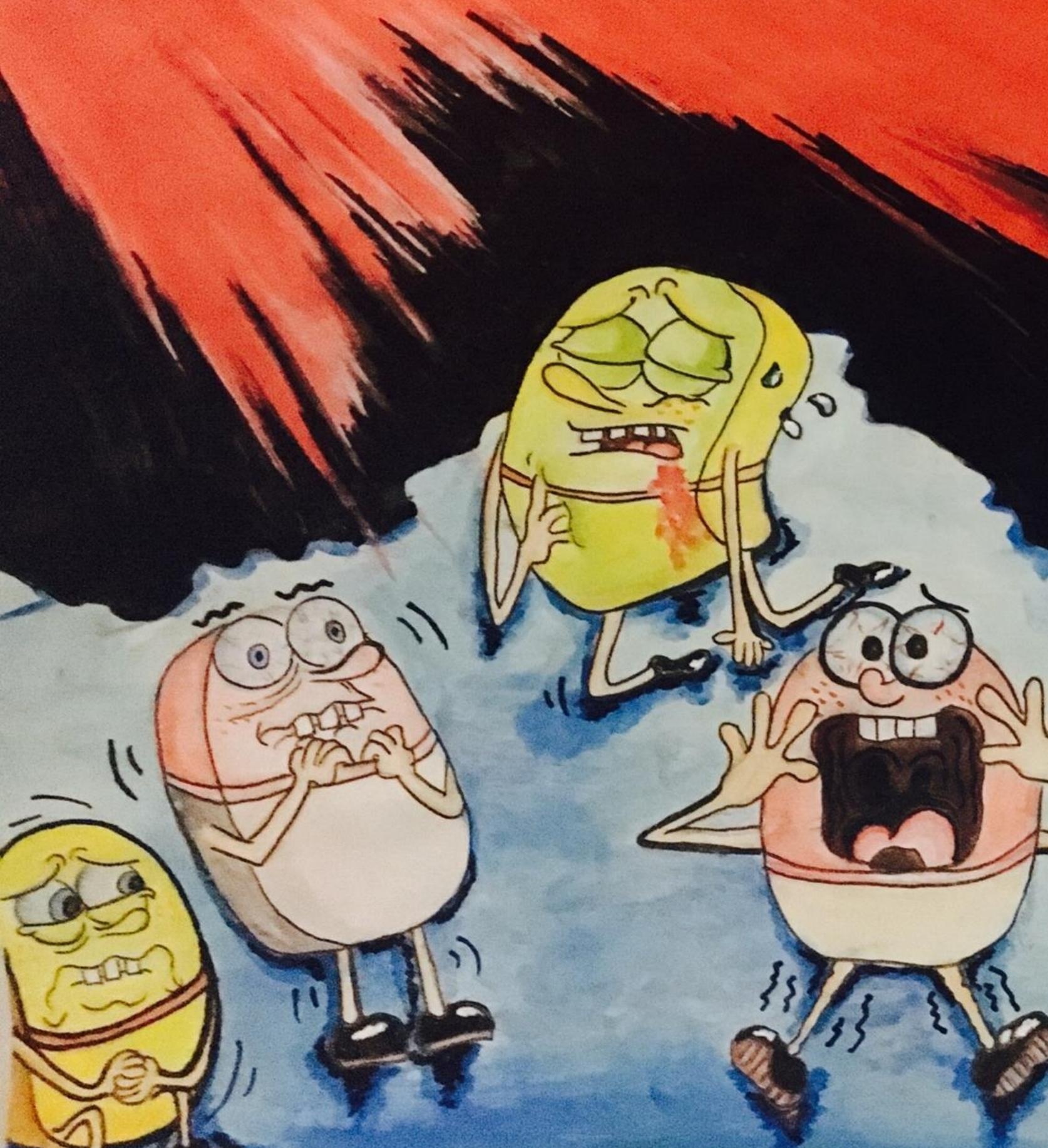

